\title{
Nickel/Photoredox Dual Catalytic Cross-Coupling of Alkyl and Amidyl Radicals to Construct $\mathrm{C}\left(\mathrm{sp}^{3}\right)-\mathrm{N}$ Bonds
}

\author{
Shaofang Zhou ${ }^{\dagger}$ Kang Lv ${ }^{\dagger \dagger}$, Rui Fu๋, Changlei Zhu ${ }^{\dagger}$, and Xiaoguang Bao ${ }^{\dagger *}$ \\ ${ }^{\dagger}$ Innovation Center for Chemical Sciences, College of Chemistry, Chemical Engineering and Materials \\ Science, Soochow University, 199 Ren-Ai Road, Suzhou Industrial Park, Suzhou, Jiangsu 215123, China. \\ ¥Department of Chemistry and Chemical Engineering, Jining University, Qufu, Shandong 273155, China. \\ ${ }^{\circledR}$ Email: $\underline{x g b a o @ s u d a . e d u . c n}$
}

Table of Contents

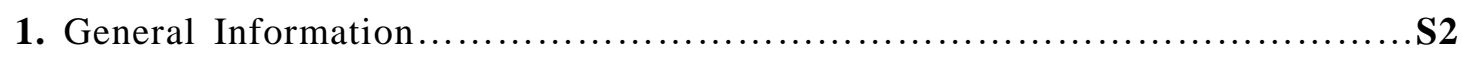

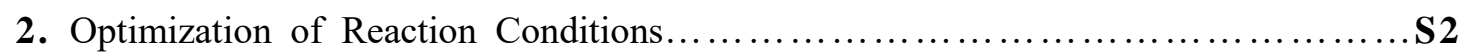

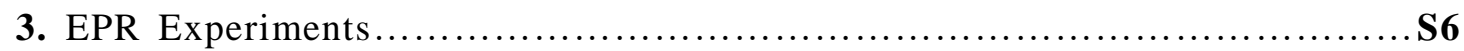

4. Stern-Volmer Quenching Experiments..................................S7

5. Computational Studies................................................... 11

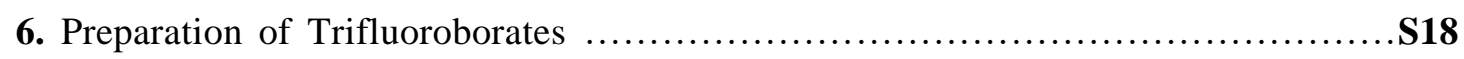

7. Synthesis and Characterization of Products.................................

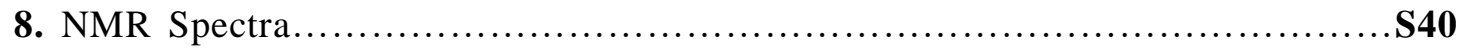

9. Cartesian Coordinates and Energies .................................... 101

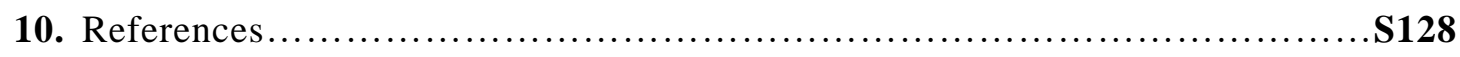




\section{General Information}

All the reactions were carried out in oven dried glassware under an argon atmosphere unless otherwise stated. Analytical grade solvents commercially available reagents were purchased from commercial sources and used directly without further purification unless otherwise stated. Flash column chromatography was performed with silica gel (300-400 mesh). NMR spectra were recorded on Varian Inova-600 MHz, Inova$400 \mathrm{MHz}$, Bruker DRX-400 spectrometer. Data were reported as chemical shifts in ppm relative to TMS (0.00 ppm) for ${ }^{1} \mathrm{H}$ and $\mathrm{CDCl}_{3}(77.2 \mathrm{ppm})$, DMSO- $\mathrm{d}_{6}(40.0 \mathrm{ppm})$ for ${ }^{13} \mathrm{C}$, respectively. The abbreviations used for explaining the multiplicities were as follows: $\mathrm{s}=$ singlet, $\mathrm{d}=$ doublet, $\mathrm{t}=$ triplet, $\mathrm{q}=$ quartet, $\mathrm{m}=$ multiplet, br $=$ broad. IR spectra were recorded using a Bruker Vertex 70 instrument. High resolution mass spectra (ESI HRMS) were recorded on a Bruker micrOTOF-Q instrument or Agilent 1200/6220 instrument by the instrumentation center of Department of Chemistry, Soochow University. Melting points were determined with a WRR melting point instrument.

\section{Optimization of Reaction Conditions}

\section{Table S1. Solvent screening.}

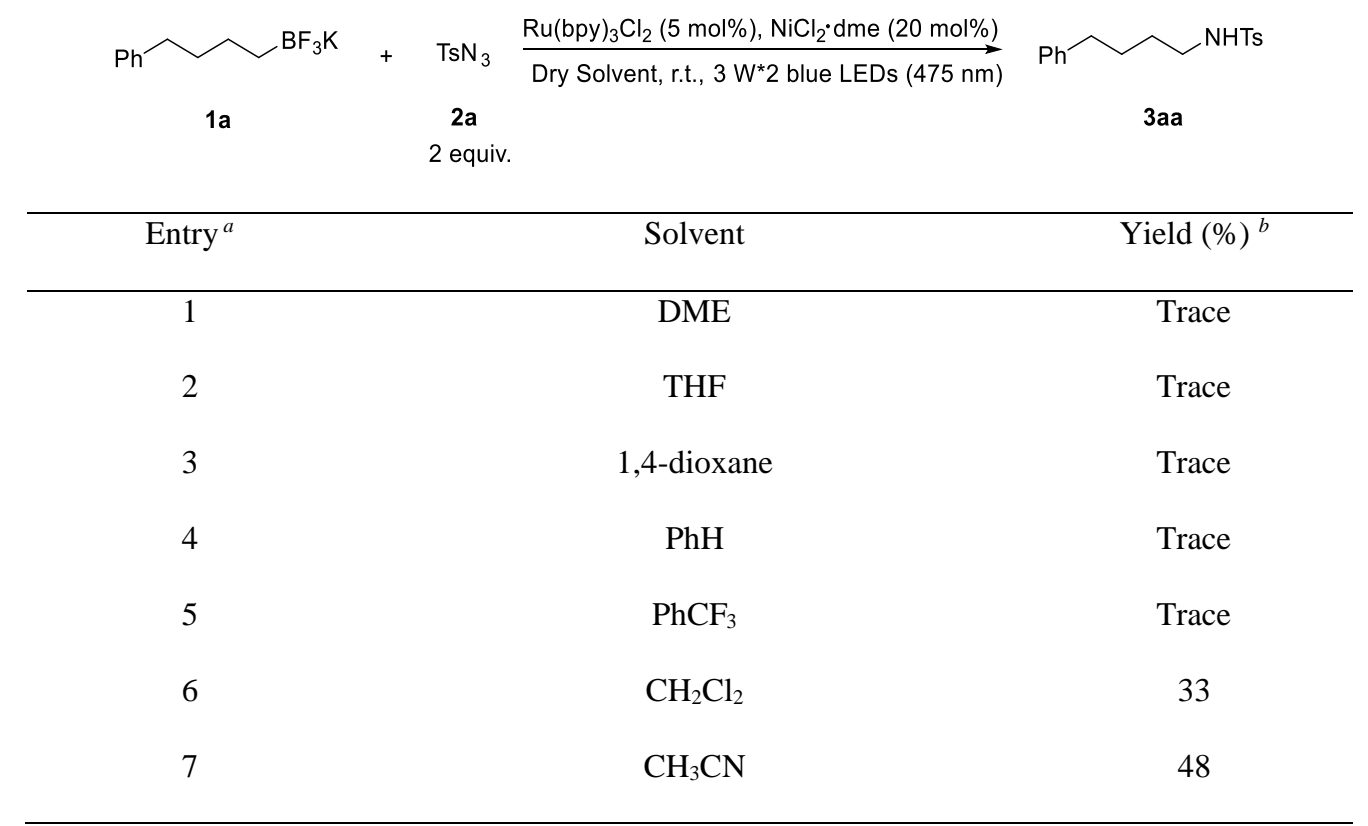

${ }^{a}$ Reaction conditions: $1 \mathbf{a}(0.1 \mathrm{mmol}), \mathbf{2 a}(0.2 \mathrm{mmol}), \mathrm{MeCN}(1 \mathrm{~mL})$, argon, r.t., irradiation with $3 \mathrm{~W} \times 2$ blue LEDs. ${ }^{b}$ Determined by ${ }^{1} \mathrm{H}$ NMR of the crude reaction mixture using an internal standard. 
Table S2. Optimization of photocatalyst and transition metal catalyst. ${ }^{a}$

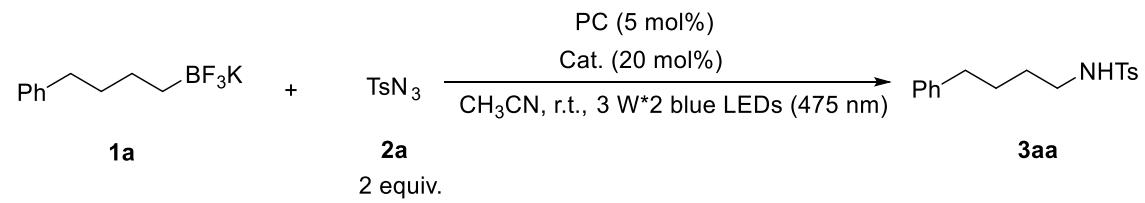

\begin{tabular}{|c|c|c|c|}
\hline Entry & $\mathrm{PC}$ & Cat. & Yield $(\%)^{b}$ \\
\hline 1 & $\operatorname{Ir}(\mathrm{ppy})_{3}$ & $\mathrm{NiCl}_{2} \cdot \mathrm{dme}$ & N.D. \\
\hline 2 & $\operatorname{Ir}\left[\mathrm{dF}\left(\mathrm{CF}_{3}\right) \mathrm{ppy}\right]_{2}(\mathrm{dtbbpy}) \mathrm{PF}_{6}$ & $\mathrm{NiCl}_{2} \cdot \mathrm{dme}$ & N.D. \\
\hline 3 & $\operatorname{Ir}(\mathrm{ppy})_{2}(\mathrm{dtbbpy}) \mathrm{PF}_{6}$ & $\mathrm{NiCl}_{2} \cdot \mathrm{dme}$ & N.D. \\
\hline 4 & $\mathrm{Ru}(\mathrm{bpy})_{3}\left(\mathrm{PF}_{6}\right)_{2}$ & $\mathrm{NiCl}_{2} \cdot \mathrm{dme}$ & 53 \\
\hline 5 & $\mathrm{Ru}(\mathrm{bpy})_{3} \mathrm{Cl}_{2}$ & $\mathrm{NiCl}_{2}$ & 35 \\
\hline 6 & $\mathrm{Ru}(\mathrm{bpy})_{3}\left(\mathrm{PF}_{6}\right)_{2}$ & $\mathrm{NiBr}_{2}$ & N.D. \\
\hline 7 & $\mathrm{Ru}(\mathrm{bpy})_{3}\left(\mathrm{PF}_{6}\right)_{2}$ & $\mathrm{NiI}_{2}$ & N.D. \\
\hline 8 & $\mathrm{Ru}(\mathrm{bpy})_{3}\left(\mathrm{PF}_{6}\right)_{2}$ & $\mathrm{Ni}(\mathrm{OTf})_{2}$ & 39 \\
\hline 9 & $\mathrm{Ru}(\mathrm{bpy})_{3}\left(\mathrm{PF}_{6}\right)_{2}$ & $\mathrm{Ni}(\mathrm{acac})_{2}$ & Trace \\
\hline 10 & $\mathrm{Ru}(\mathrm{bpy})_{3}\left(\mathrm{PF}_{6}\right)_{2}$ & $\mathrm{Ni}(\mathrm{OAc})_{2}$ & Trace \\
\hline 11 & $\mathrm{Ru}(\mathrm{bpy})_{3}\left(\mathrm{PF}_{6}\right)_{2}$ & {$\left[\mathrm{Cu}\left(\mathrm{CH}_{3} \mathrm{CN}\right)_{4}\right] \mathrm{BF}_{4}$} & 35 \\
\hline 12 & $\mathrm{Ru}(\mathrm{bpy})_{3}\left(\mathrm{PF}_{6}\right)_{2}$ & $\mathrm{Cu}(\mathrm{OAc})_{2}$ & 8 \\
\hline 13 & $\mathrm{Ru}(\mathrm{bpy})_{3}\left(\mathrm{PF}_{6}\right)_{2}$ & $\mathrm{Cu}(\mathrm{OTf})_{2}$ & 5 \\
\hline 14 & $\mathrm{Ru}(\mathrm{bpy})_{3}\left(\mathrm{PF}_{6}\right)_{2}$ & $\mathrm{Cu}(\mathrm{acac})_{2}$ & 9 \\
\hline 15 & $\mathrm{Ru}(\mathrm{bpy})_{3}\left(\mathrm{PF}_{6}\right)_{2}$ & $\mathrm{CuCl}_{2}$ & Trace \\
\hline 16 & $\mathrm{Ru}(\mathrm{bpy})_{3}\left(\mathrm{PF}_{6}\right)_{2}$ & $\mathrm{CuBr}_{2}$ & Trace \\
\hline 17 & $\mathrm{Ru}(\mathrm{bpy})_{3}\left(\mathrm{PF}_{6}\right)_{2}$ & $\mathrm{CuI}$ & 8 \\
\hline 18 & $\mathrm{Ru}(\mathrm{bpy})_{3}\left(\mathrm{PF}_{6}\right)_{2}$ & $\mathrm{Co}(\mathrm{OAc})_{2}$ & 6 \\
\hline 19 & $\mathrm{Ru}(\mathrm{bpy})_{3}\left(\mathrm{PF}_{6}\right)_{2}$ & $\mathrm{Co}(\mathrm{acac})_{3}$ & 3 \\
\hline 20 & Methylene blue & $\mathrm{NiCl}_{2} \cdot \mathrm{dme}$ & N.D. \\
\hline 21 & Eosin B & $\mathrm{NiCl}_{2} \cdot \mathrm{dme}$ & N.D. \\
\hline 22 & Eosin $Y$ & $\mathrm{NiCl}_{2} \cdot \mathrm{dme}$ & N.D. \\
\hline 23 & Mes-Acr ${ }^{+} \mathrm{ClO}_{4}^{-}$ & $\mathrm{NiCl}_{2} \cdot \mathrm{dme}$ & N.D. \\
\hline 24 & TBADT & $\mathrm{NiCl}_{2} \cdot \mathrm{dme}$ & N.D. \\
\hline $25^{c}$ & $\mathrm{Ru}(\mathrm{bpy})_{3}\left(\mathrm{PF}_{6}\right)_{2}$ & $\mathrm{NiCl}_{2} \cdot \mathrm{dme}$ & 58 \\
\hline
\end{tabular}




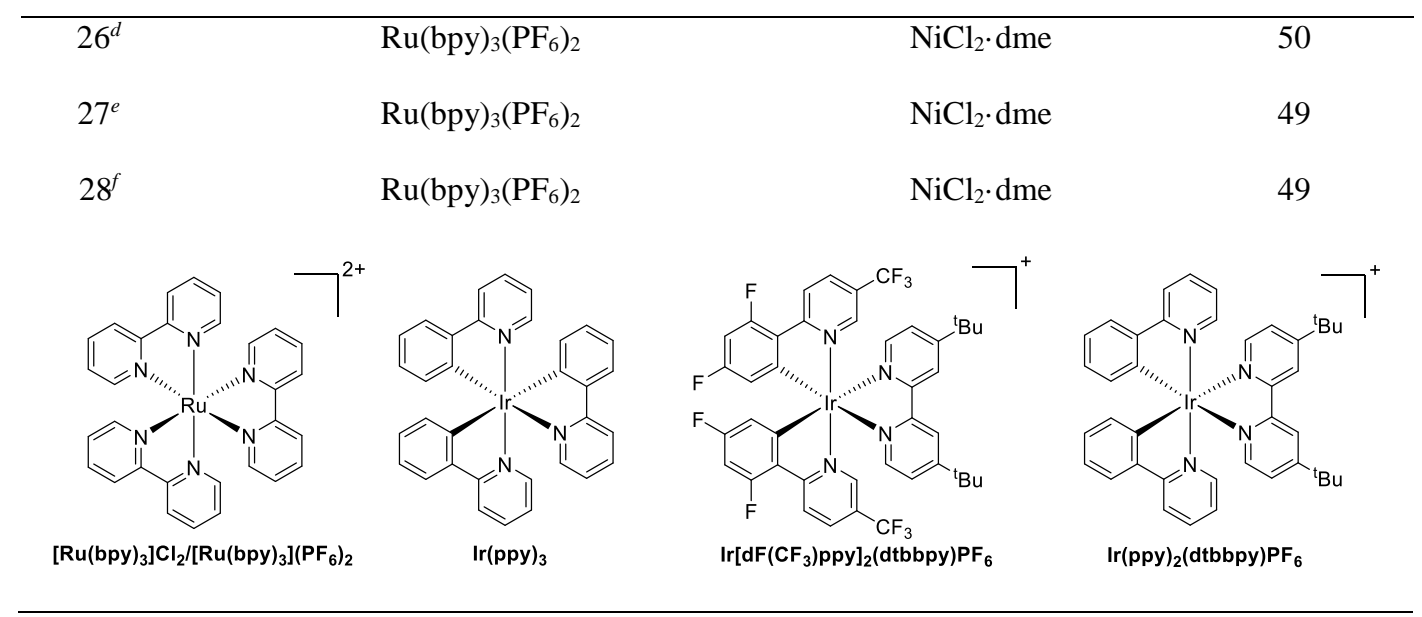

${ }^{a}$ Reaction conditions: 1a $(0.1 \mathrm{mmol}), \mathbf{2 a}(0.2 \mathrm{mmol}), \mathrm{MeCN}(1 \mathrm{~mL})$, argon, r. t., irradiation with $3 \mathrm{~W} \times$ 2 blue LEDs. ${ }^{b}$ Determined by ${ }^{1} \mathrm{H}$ NMR of the crude reaction mixture using an internal standard. ${ }^{c} 50$ mol\% biacetyl was added. ${ }^{d}$ benzophenone was added instead of biacetyl. ${ }^{e}$ anthraquinone was added instead of biacetyl. ${ }^{f}$ 9-fluorenone was added instead of biacetyl.

Table S3. Optimization of the dosage of photocatalyst, transition metal catalyst, and additive.$^{a}$

\begin{tabular}{|c|c|c|c|c|}
\hline \multirow{3}{*}{$1 \mathrm{a}$} & $\mathrm{BF}_{3} \mathrm{~K}$ & \multicolumn{2}{|c|}{$\begin{array}{c}\mathrm{Ru}(\mathrm{bpy})_{3}\left(\mathrm{PF}_{6}\right)_{2}(\mathrm{X} \mathrm{mol} \%) \\
\underset{\mathrm{NiCl}_{2} \cdot \mathrm{dme}(\mathrm{X} \mathrm{mol} \%) \text {, biacetyl }(\mathrm{X} \mathrm{mol} \%)}{\underset{\mathrm{CH}_{3} \mathrm{CN} \text {, r.t., } 3 \mathrm{~W}^{*} 2 \text { purple LEDs }(400 \mathrm{~nm})}{P}} \mathrm{Ph}^{-}\end{array}$} & \multirow[b]{2}{*}{ 3aa } \\
\hline & \multicolumn{2}{|c|}{2 equiv. } & & \\
\hline & $\mathrm{Ru}(\mathrm{bpy})_{3} \mathrm{PF}_{6}$ & $\mathrm{NiCl}_{2} \cdot \mathrm{dme}$ & biacetyl & Yield $(\%)^{b}$ \\
\hline 1 & $10 \mathrm{~mol} \%$ & $20 \mathrm{~mol} \%$ & $20 \mathrm{~mol} \%$ & 74 \\
\hline 2 & $10 \mathrm{~mol} \%$ & $20 \mathrm{~mol} \%$ & $50 \mathrm{~mol} \%$ & 81 \\
\hline 3 & $10 \mathrm{~mol} \%$ & $20 \mathrm{~mol} \%$ & $100 \mathrm{~mol} \%$ & 72 \\
\hline 4 & $5 \mathrm{~mol} \%$ & $20 \mathrm{~mol} \%$ & $50 \mathrm{~mol} \%$ & 60 \\
\hline 5 & $1 \mathrm{~mol} \%$ & $20 \mathrm{~mol} \%$ & $50 \mathrm{~mol} \%$ & 38 \\
\hline 6 & $10 \mathrm{~mol} \%$ & $15 \mathrm{~mol} \%$ & $50 \mathrm{~mol} \%$ & 68 \\
\hline 7 & $10 \mathrm{~mol} \%$ & $10 \mathrm{~mol} \%$ & $50 \mathrm{~mol} \%$ & 48 \\
\hline 8 & $10 \mathrm{~mol} \%$ & $5 \mathrm{~mol} \%$ & $50 \mathrm{~mol} \%$ & 20 \\
\hline
\end{tabular}

\footnotetext{
${ }^{a}$ Reaction conditions: 1a $(0.1 \mathrm{mmol}), \mathbf{2 a}(0.2 \mathrm{mmol}), \mathrm{MeCN}(1 \mathrm{~mL})$, argon, r.t., irradiation with $3 \mathrm{~W}$ $\times 2$ purple LEDs. ${ }^{b}$ Determined by ${ }^{1} \mathrm{H}$ NMR of the crude reaction mixture using an internal standard.
} 
Table S4. The reaction of $1 \mathrm{a}$ with other organic azides.

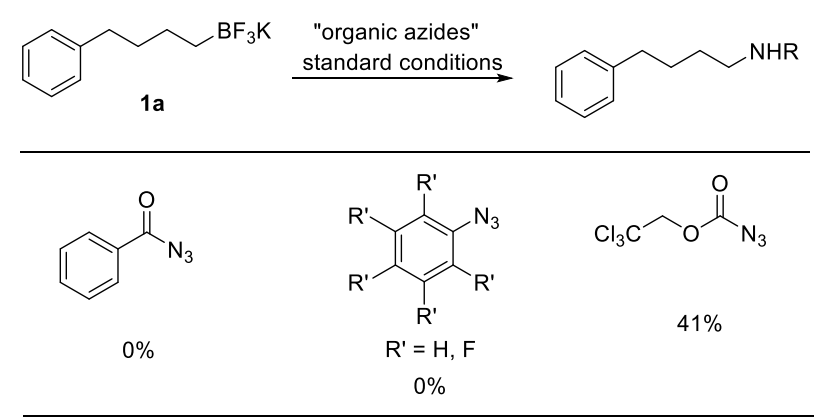

Table S5. Optimization of the reaction of $1 \mathrm{a}$ with $6 \mathbf{a}^{a}$

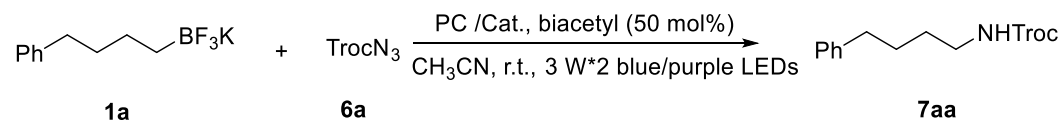

$1 a$

$6 a$ Yield (\%)

\begin{tabular}{|c|c|c|}
\hline Entry & Conditions & Yield $(\%)^{b}$ \\
\hline 1 & $\begin{array}{c}\mathrm{Ru}(\mathrm{bpy})_{3}\left(\mathrm{PF}_{6}\right)_{2}(10 \mathrm{~mol} \%), \mathrm{NiCl}_{2} \cdot \mathrm{dme}(20 \mathrm{~mol} \%) \\
2 \text { eq. } 6 \mathbf{a}, 400 \mathrm{~nm} \text { light }\end{array}$ & 41 \\
\hline 2 & $\begin{array}{c}\mathrm{Ru}(\mathrm{bpy})_{3}\left(\mathrm{PF}_{6}\right)_{2}(10 \mathrm{~mol} \%), \mathrm{NiCl}_{2} \cdot \mathrm{dme}(20 \mathrm{~mol} \%) \\
2.5 \text { eq. 6a, } 400 \mathrm{~nm} \text { light }\end{array}$ & 52 \\
\hline 3 & $\begin{array}{c}\mathrm{Ru}(\mathrm{bpy})_{3}\left(\mathrm{PF}_{6}\right)_{2}(10 \mathrm{~mol} \%), \mathrm{NiCl}_{2} \cdot \mathrm{dme}(20 \mathrm{~mol} \%) \\
3 \text { eq. } \mathbf{6 a}, 400 \mathrm{~nm} \text { light }\end{array}$ & 64 \\
\hline 4 & $\begin{array}{c}\mathrm{Ru}(\mathrm{bpy})_{3}\left(\mathrm{PF}_{6}\right)_{2}(10 \mathrm{~mol} \%), \mathrm{NiCl}_{2} \cdot \mathrm{dme}(20 \mathrm{~mol} \%) \\
3 \text { eq. } \mathbf{6 a}, 475 \mathrm{~nm} \text { light }\end{array}$ & 68 \\
\hline 5 & $\begin{array}{c}\mathrm{Ru}(\mathrm{bpy})_{3}\left(\mathrm{PF}_{6}\right)_{2}(2.5 \mathrm{~mol} \%), \mathrm{NiCl}_{2} \cdot \mathrm{dme}(10 \mathrm{~mol} \%) \\
3 \text { eq. } \mathbf{6 a}, 475 \mathrm{~nm} \text { light }\end{array}$ & 84 \\
\hline 6 & $\begin{array}{c}\mathrm{Ru}(\mathrm{bpy})_{3}\left(\mathrm{PF}_{6}\right)_{2}(2.5 \mathrm{~mol} \%), \mathrm{Ni}(\mathrm{OTf})_{2}(20 \mathrm{~mol} \%) \\
3 \text { eq. } \mathbf{6 a}, 475 \mathrm{~nm} \text { light }\end{array}$ & 73 \\
\hline 7 & $\begin{array}{c}\mathrm{Ru}(\mathrm{bpy})_{3}\left(\mathrm{PF}_{6}\right)_{2}(10 \mathrm{~mol} \%), \mathrm{Ni}\left(\mathrm{PPh}_{3}\right)_{2} \mathrm{Cl}_{2}(10 \mathrm{~mol} \%) \\
3 \text { eq. } \mathbf{6 a}, 475 \mathrm{~nm} \text { light }\end{array}$ & 70 \\
\hline 8 & $\begin{array}{c}\mathrm{Ru}(\mathrm{bpy})_{3}\left(\mathrm{PF}_{6}\right)_{2}(10 \mathrm{~mol} \%), \mathrm{Ni}(\mathrm{cod})_{2}(10 \mathrm{~mol} \%) \\
3 \text { eq. 6a, } 475 \mathrm{~nm} \text { light }\end{array}$ & 0 \\
\hline 9 & $\begin{array}{c}\mathrm{Ru}(\mathrm{bpy})_{3}\left(\mathrm{PF}_{6}\right)_{2}(10 \mathrm{~mol} \%), \mathrm{Ni}\left(\mathrm{PPh}_{3}\right)_{4}(10 \mathrm{~mol} \%) \\
3 \text { eq. } \mathbf{6 a}, 475 \mathrm{~nm} \text { light }\end{array}$ & 0 \\
\hline
\end{tabular}

${ }^{a}$ Reaction conditions: 1a $(0.1 \mathrm{mmol}), \mathbf{6 a}(0.2 \sim 0.3 \mathrm{mmol}), \mathrm{MeCN}(1 \mathrm{~mL})$, argon, r. t., irradiation with $3 \mathrm{~W}$ $\times 2$ blue/purple LEDs. ${ }^{b}$ Determined by ${ }^{1} \mathrm{H}$ NMR of the crude reaction mixture using an internal standard. 


\section{EPR Experiments}

To confirm the existence of radicals in the reaction, we performed Electron Paramagnetic Resonance (EPR) experiments using the radical trapping reagent DMPO. EPR spectra were recorded on a JES-X320 spectrometer (X-band) by the instrumentation center of Department of Chemistry, Soochow University. The spectra were measured at room temperature. The radical adducts were further confirmed by ESI-HRMS.

3.1 To a Schlenk tube, $\left[\mathrm{Ru}(\mathrm{bpy})_{3}\right]\left(\mathrm{PF}_{6}\right)_{2}(8.6 \mathrm{mg}, 0.01 \mathrm{mmol}), \mathrm{NiCl}_{2} \cdot \mathrm{dme}(4.3 \mathrm{mg}, 0.02 \mathrm{mmol})$ was taken with the solvent $\mathrm{MeCN}(1 \mathrm{~mL})$ under argon atmosphere. The reaction mixture was irradiated with purple LEDs $(3 \mathrm{~W} \times 2)$ for $5 \mathrm{~min}$. The resulting reaction mixture was analyzed by EPR. No obvious EPR signal was found in Figure S1.

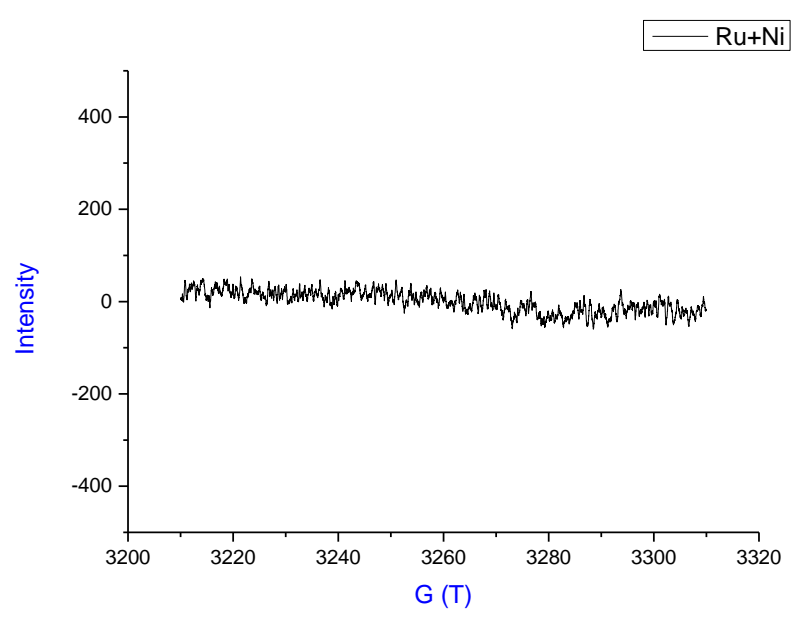

Figure S1. EPR spectrum for the mixture of the photocatalyst and transition metal catalyst.

3.2 To a Schlenk tube, 1a $(24 \mathrm{mg}, 0.1 \mathrm{mmol}), \mathbf{2 a}(30 \mu \mathrm{L}, 0.2 \mathrm{mmol}),\left[\mathrm{Ru}(\mathrm{bpy})_{3}\right]\left(\mathrm{PF}_{6}\right)_{2}(8.6 \mathrm{mg}, 0.01 \mathrm{mmol})$ and $\mathrm{NiCl}_{2} \cdot \mathrm{dme}(4.3 \mathrm{mg}, 0.02 \mathrm{mmol})$ were taken with the solvent $\mathrm{MeCN}(1 \mathrm{~mL})$ under argon atmosphere. The reaction mixture was irradiated with purple LEDs $(3 \mathrm{~W} \times 2)$ for $5 \mathrm{~min}$, then DMPO (1 eq.) was added. The resulting reaction mixture was analyzed by EPR (Figure S2). Although mixed EPR signals of radical adducts were recorded, it is very hard to distinguish between 1aa and 2aa. Nevertheless, the proposed radical adducts 1aa and 2aa were consistent with the results of ESI-HRMS (Figure S3). Therefore, we can conclude the formation of carbon center radical and nitrogen center radical in this system.

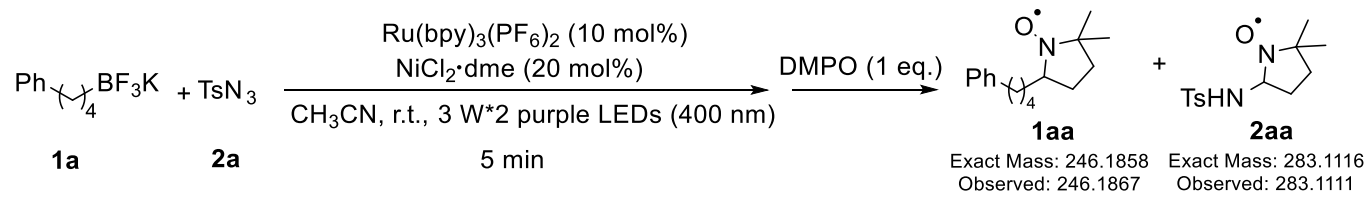



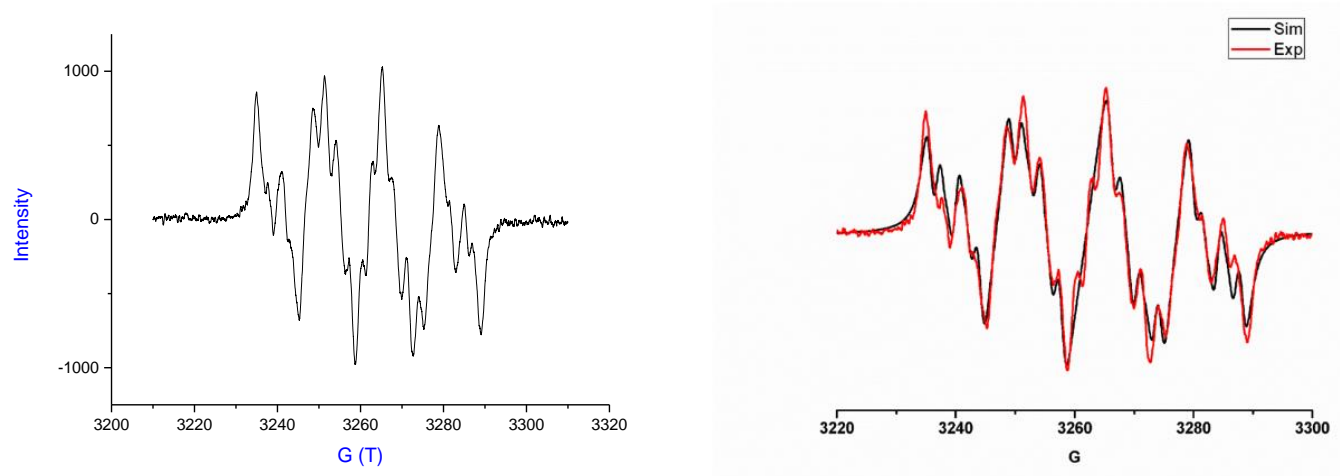

Figure S2. EPR spectrum (trapped by DMPO) for the mixture of $\mathbf{1 a}$ and $\mathbf{2 a}$ in the presence of the photocatalyst and transition metal catalyst.

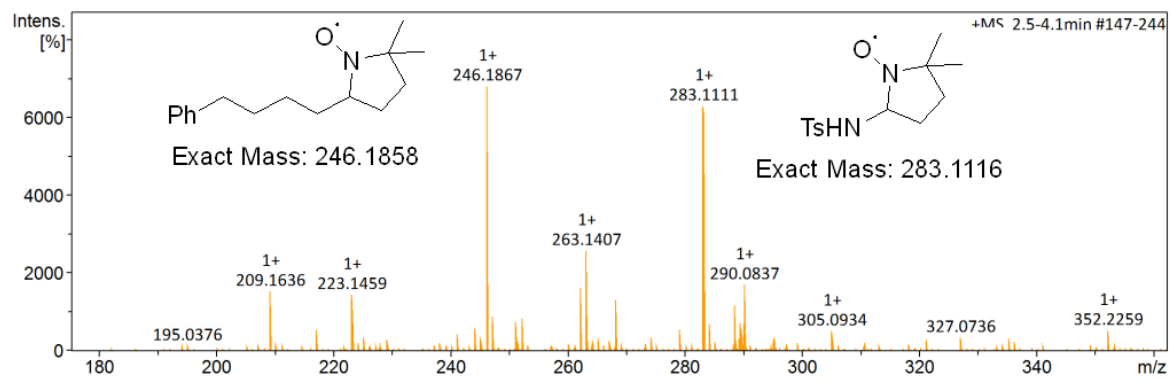

Figure S3. The proposed radical adducts 1aa and 2aa were consistent with the results of ESI-HRMS.

\section{Stern-Volmer Quenching Experiments}

\subsection{Quenching experiments with $\operatorname{Ru}\left(b_{p y}\right)_{3}\left(P_{6}\right)_{2}$}

F-2700 Fluorescence Spectrophotometer was employed to collect the spectra. The solution of $\mathrm{Ru}(\mathrm{bpy})_{3}\left(\mathrm{PF}_{6}\right)_{2}$ in acetone was excited with $\lambda_{\mathrm{ex}}=470 \mathrm{~nm}$ and the maximum emission $\lambda_{\mathrm{em}}$ was found at 610 nm. The emission spectrum of a $0.5 \mathrm{mM}$ solution of $\mathrm{Ru}(\mathrm{bpy})_{3}\left(\mathrm{PF}_{6}\right)_{2}$ was collected. Emission quenching experiments were performed with substrates $\mathbf{1 a}, \mathbf{2 a}, \mathbf{4 a}, \mathbf{4 r}$, and catalyst $\mathrm{NiCl}_{2} \cdot \mathrm{dme}$ at different concentration $(0,0.5,1.0,2.0,4.0 \mathrm{mM})$. The results showed that alkyltrifluoroborates $(\mathbf{1 a}, \mathbf{4 a}, \mathbf{4 r})$ and azide $\mathbf{2 a}$ could not quench the luminescence. Only $\mathrm{NiCl}_{2} \cdot \mathrm{dme}$ is capable of quenching the excited state of $\mathrm{Ru}(\mathrm{bpy})_{3}\left(\mathrm{PF}_{6}\right)_{2}$. 


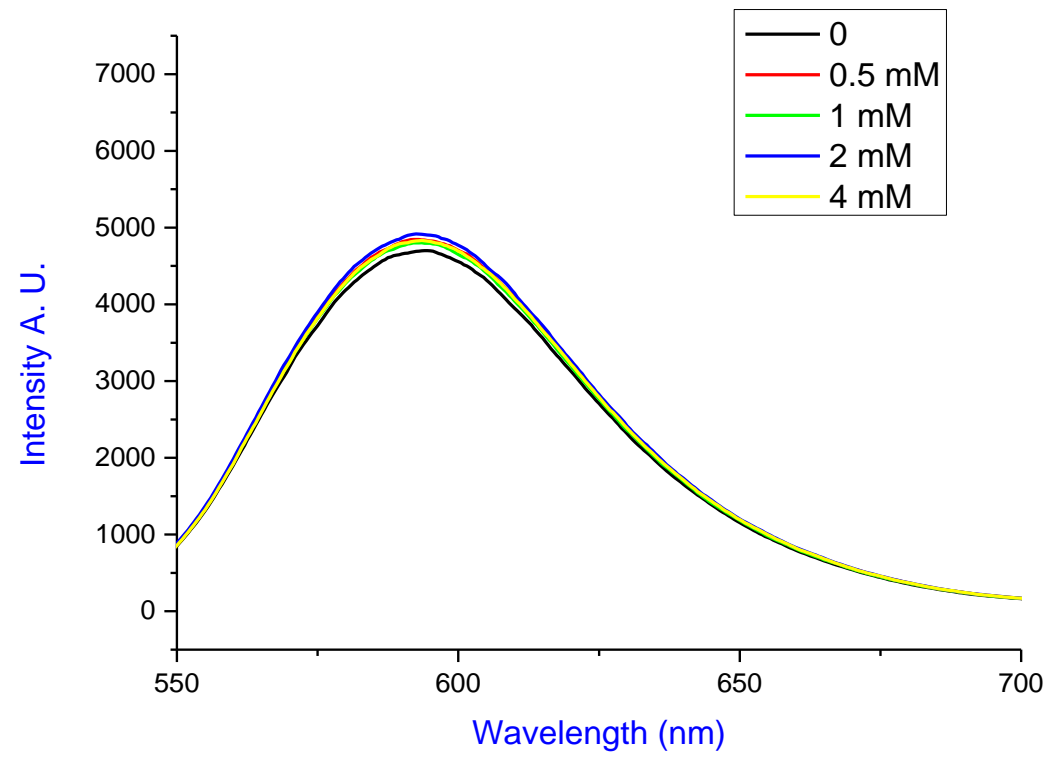

Figure S4. Phosphorescence quenching of the emission of $\mathrm{Ru}(\mathrm{bpy})_{3}\left(\mathrm{PF}_{6}\right)_{2}\left(0.5 \mathrm{mM}\right.$ in $\left.\mathrm{CH}_{3} \mathrm{CN}\right)$ at room temperature with different concentration of $\mathbf{2 a}\left(\lambda_{\mathrm{ex}}=470 \mathrm{~nm}\right)$.

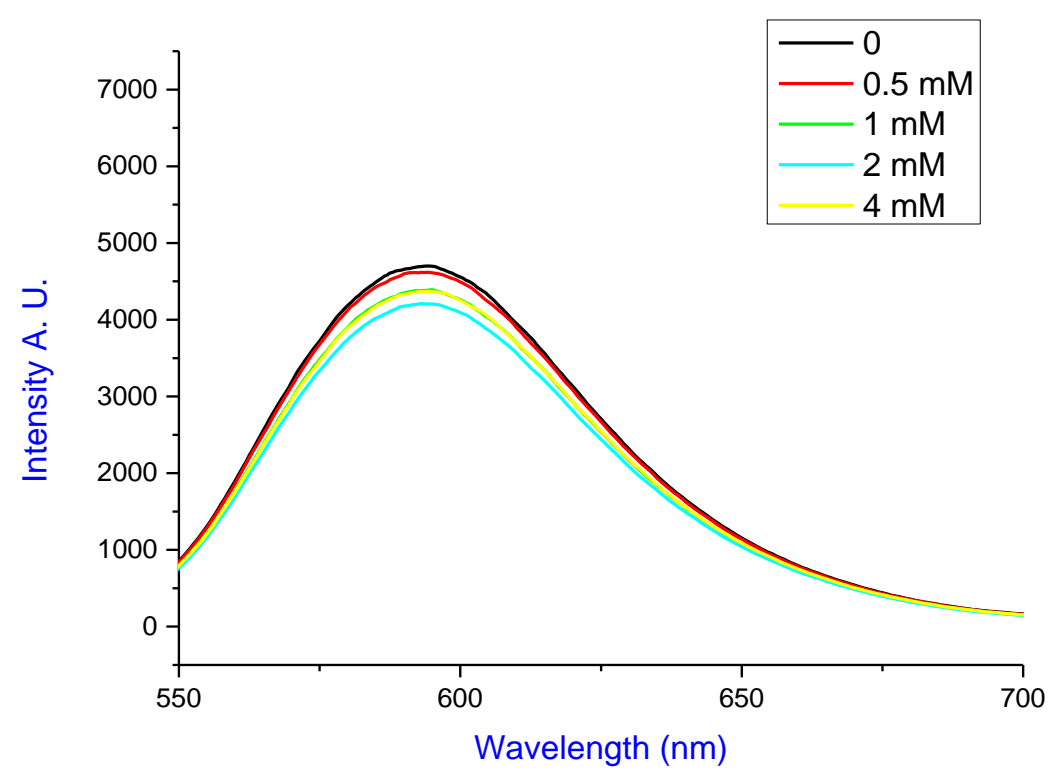

Figure S5. Phosphorescence quenching of the emission of $\mathrm{Ru}(\mathrm{bpy})_{3}\left(\mathrm{PF}_{6}\right)_{2}\left(0.5 \mathrm{mM}\right.$ in $\left.\mathrm{CH}_{3} \mathrm{CN}\right)$ at room temperature with different concentration of $\mathbf{1 a}\left(\lambda_{\mathrm{ex}}=470 \mathrm{~nm}\right)$. 


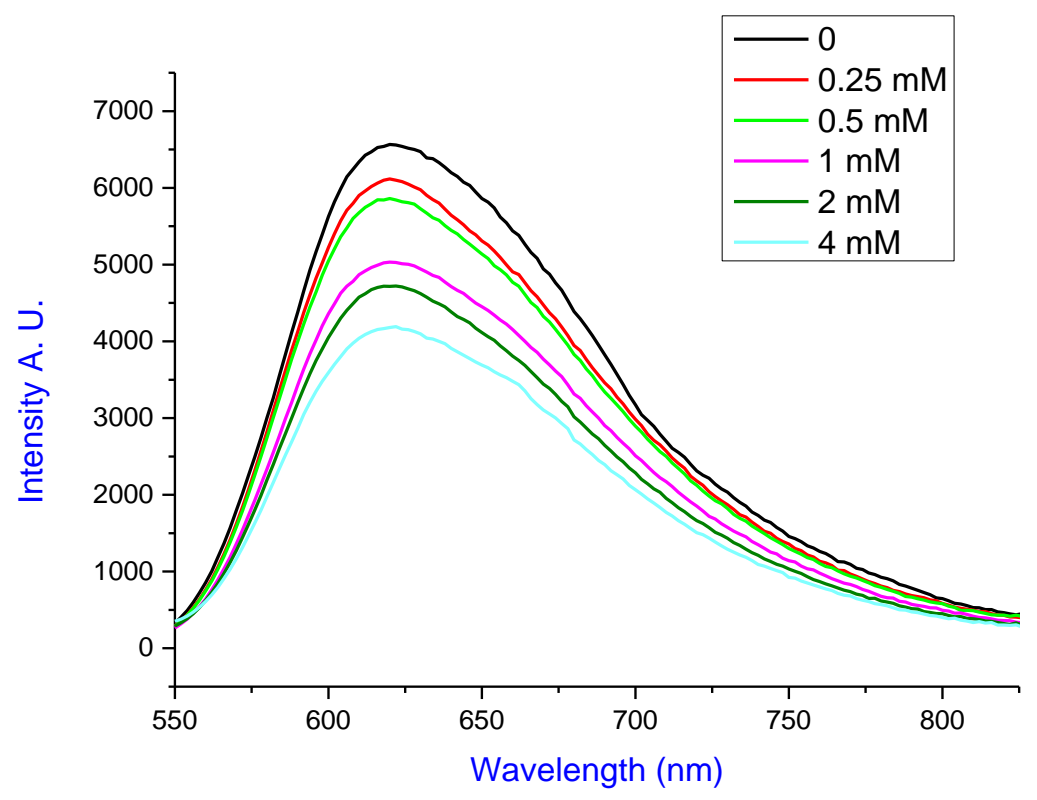

Figure S6. Phosphorescence quenching of the emission of $\mathrm{Ru}(\mathrm{bpy})_{3}\left(\mathrm{PF}_{6}\right)_{2}\left(0.5 \mathrm{mM}\right.$ in $\left.\mathrm{CH}_{3} \mathrm{CN}\right)$ at room temperature with different concentration of $\mathrm{NiCl}_{2} \cdot \mathrm{dme}\left(\lambda_{\mathrm{ex}}=470 \mathrm{~nm}\right)$.

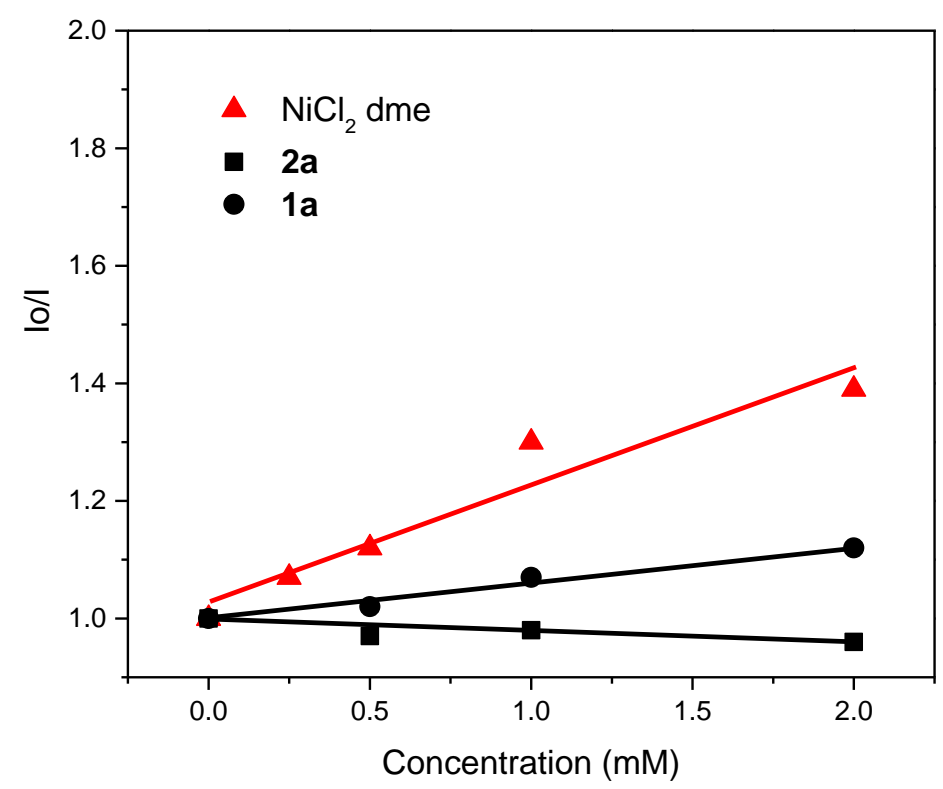

Figure S7. Stern-Volmer plot of $\mathrm{Ru}(\mathrm{bpy})_{3}\left(\mathrm{PF}_{6}\right)_{2}(0.5 \mathrm{mM})$ at different concentrations of $\mathrm{NiCl}_{2} \cdot \mathrm{dme}, \mathbf{1 a}$ and $2 a$. 


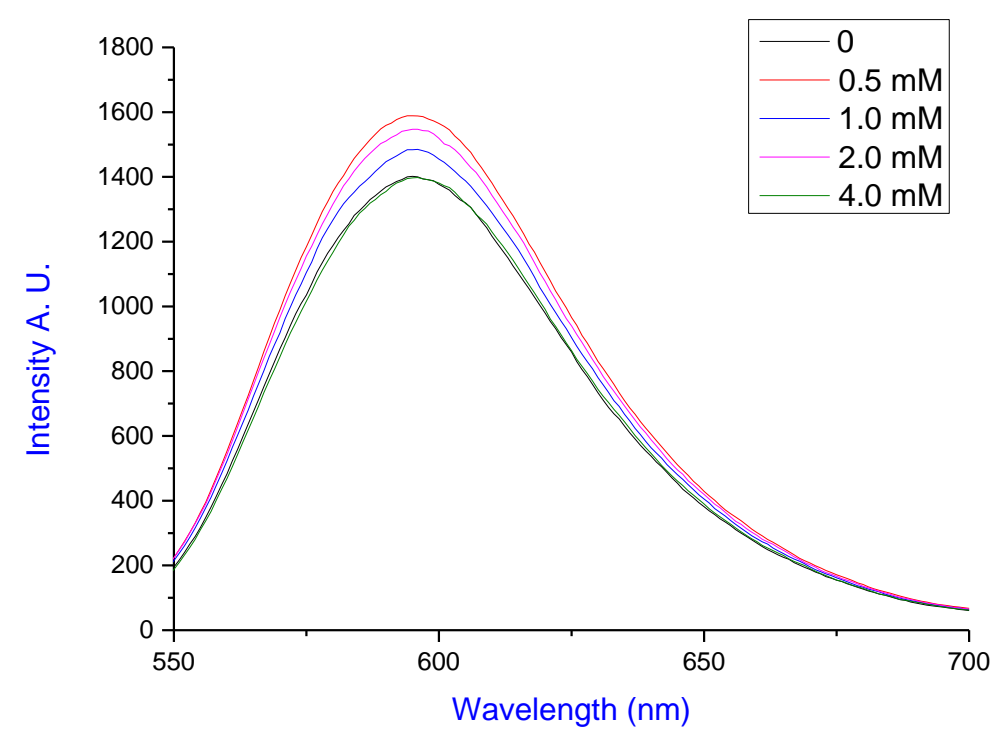

Figure S8. Phosphorescence quenching of the emission of $\mathrm{Ru}(\mathrm{bpy})_{3}\left(\mathrm{PF}_{6}\right)_{2}\left(0.5 \mathrm{mM}\right.$ in $\left.\mathrm{CH}_{3} \mathrm{CN}\right)$ at room temperature with different concentration of $\mathbf{4 a}\left(\lambda_{\mathrm{ex}}=470 \mathrm{~nm}\right)$.

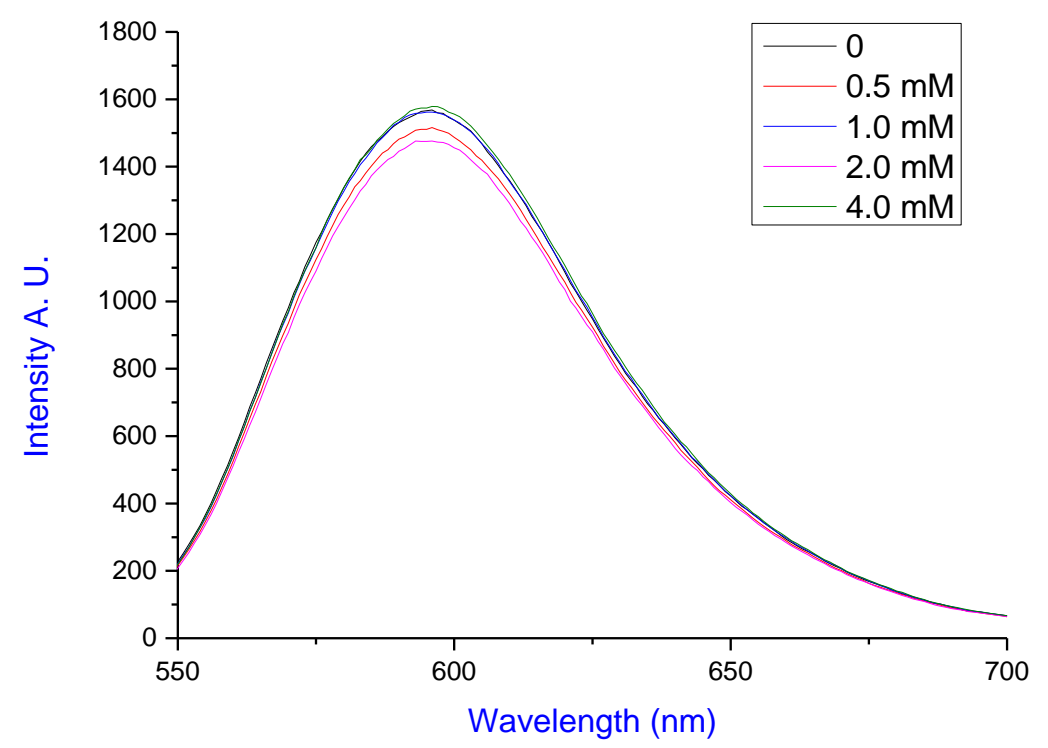

Figure S9. Phosphorescence quenching of the emission of $\mathrm{Ru}(\mathrm{bpy})_{3}\left(\mathrm{PF}_{6}\right)_{2}\left(0.5 \mathrm{mM}\right.$ in $\left.\mathrm{CH}_{3} \mathrm{CN}\right)$ at room temperature with different concentration of $4 \mathbf{r}\left(\lambda_{\mathrm{ex}}=470 \mathrm{~nm}\right)$. 


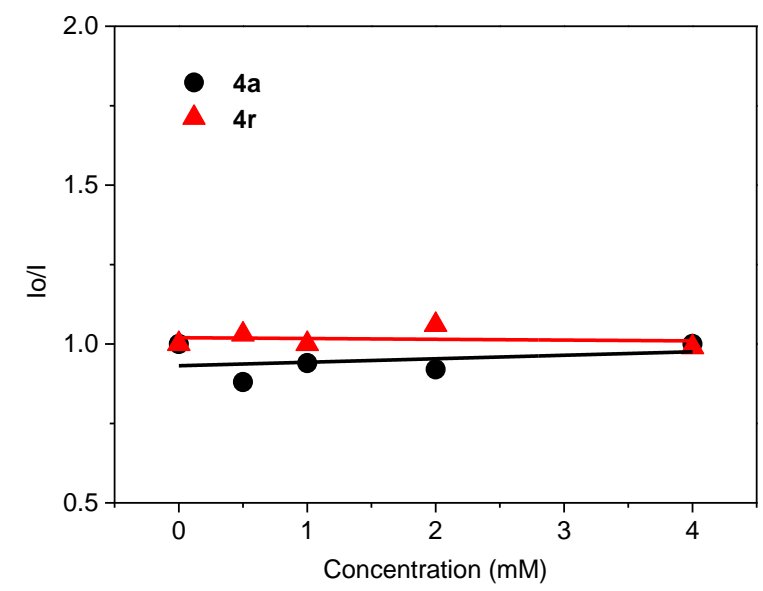

Figure S10. Stern-Volmer plot of $\mathrm{Ru}(\mathrm{bpy})_{3}\left(\mathrm{PF}_{6}\right)_{2}(0.5 \mathrm{mM})$ at different concentrations of $\mathbf{4 a}$ and $\mathbf{4 r}$.

\section{Computational Studies}

\subsection{Computational methods}

The B3LYP density functional method with Grimme-D3 correction ${ }^{1}$ was employed to carry out the computational studies. For geometry optimizations, the LANL2DZ ${ }^{2}$ pseudopotential was used for the core along with the valence electrons of $\mathrm{Ni}$ and $\mathrm{Ru}$ atoms. The $6-31+\mathrm{G}(\mathrm{d})^{3}$ basis set was used for other atoms. Vibrational frequency analyses at the same level of theory were performed on all the optimized geometries to characterize stationary points as local minima (no imaginary frequency) or transition states (one imaginary frequency). In addition, intrinsic reaction coordinate (IRC) calculations ${ }^{4}$ were used to verify that the transition state connects with appropriate reactant and product. The gas-phase Gibbs energies for all species were obtained at $298.15 \mathrm{~K}$ and 1 atm at their respective optimized structures. To consider the effect of solvation, B3LYP-D3 functional with the $\mathrm{SMD}^{5}$ continuum solvation model (in acetonitrile solvent) was used in single-point energy calculations. A larger basis set, $\mathrm{SDD}^{6}$ for $\mathrm{Ni}$ and $\mathrm{Ru}$ atoms and $6-311++\mathrm{G}(2 \mathrm{~d}, \mathrm{p})$ for the remaining atoms, was utilized for such single-point energy calculation. The solvation Gibbs energy was used for discussion and its value was obtained from the addition of solvation single-point energy and gas-phase thermal correction to Gibbs energy. All calculations were carried out with the Gaussian 09 suite of programs ${ }^{7}$.

The Marcus Theory ${ }^{8}$ was applied to calculate the kinetic barrier of single electron transfer (SET). For a SET reaction below,

$$
*[\mathrm{Ru}]^{2+}+\mathrm{R}_{1} \longrightarrow[\mathrm{SET} \longrightarrow]^{3+}+\mathrm{P}_{1}
$$

according to the Marcus-Hush equation, the solvent outer-sphere reorganization energy $\lambda_{\circ}$ could be calculated from Equations S1 and S2. 


$$
\begin{array}{cr}
\lambda_{0}=(332 \mathrm{kcal} / \mathrm{mol})\left(\frac{1}{2 a_{1}}+\frac{1}{2 a_{2}}-\frac{1}{R}\right)\left(\frac{1}{\varepsilon_{o p}}-\frac{1}{\varepsilon}\right) & \text { Equation } \mathrm{S} 1 \\
R=a_{1}+a_{2} & \text { Equation } \mathrm{S} 2
\end{array}
$$

$a_{1}$ and $a_{2}$ are the radii of the excited-state photoredox $* \mathbf{R} \mathbf{u}^{\text {II }}$ and reactant $\mathbf{R}_{\mathbf{1}}$, respectively. $\varepsilon_{o p}$ and $\varepsilon$ are the optical dielectric constant (2.25) and the static dielectric constant (35.7) of acetonitrile solvent, respectively. The radius of a molecule can be derived by calculating molecular volume via a Monte-Carlo integration as implemented in the Gaussian 09 program. For a single electron transfer reaction, the inner reorganization energies of the reactants are usually small enough and thus are neglected. Therefore, the total reorganization energy $\lambda$ is approximately equal to $\lambda_{o}$. Next, the $\Delta \mathrm{G}^{\neq}$of a single electron transfer process could be calculated from Equations $\mathrm{S} 3$ and $\mathrm{S} 4 . \Delta \mathrm{G}_{r}$ is the Gibbs energy change for a SET reaction (Equation S5).

$$
\begin{array}{cc}
\Delta G_{E T}^{\neq}=\Delta G_{0}^{\neq}\left(1+\frac{\Delta G_{r}}{4 \Delta G_{0}^{\ddagger}}\right)^{2} & \text { Equation S3 } \\
\Delta G_{0}^{\neq}=\frac{\lambda}{4} & \text { Equation S4 } \\
\Delta G_{r}=G\left([R u]^{3+}\right)+G\left(P_{1}\right)-G\left(*[R u]^{2+}\right)-G\left(R_{1}\right) & \text { Equation S5 }
\end{array}
$$

Table S6. Estimated activation barriers for SET steps

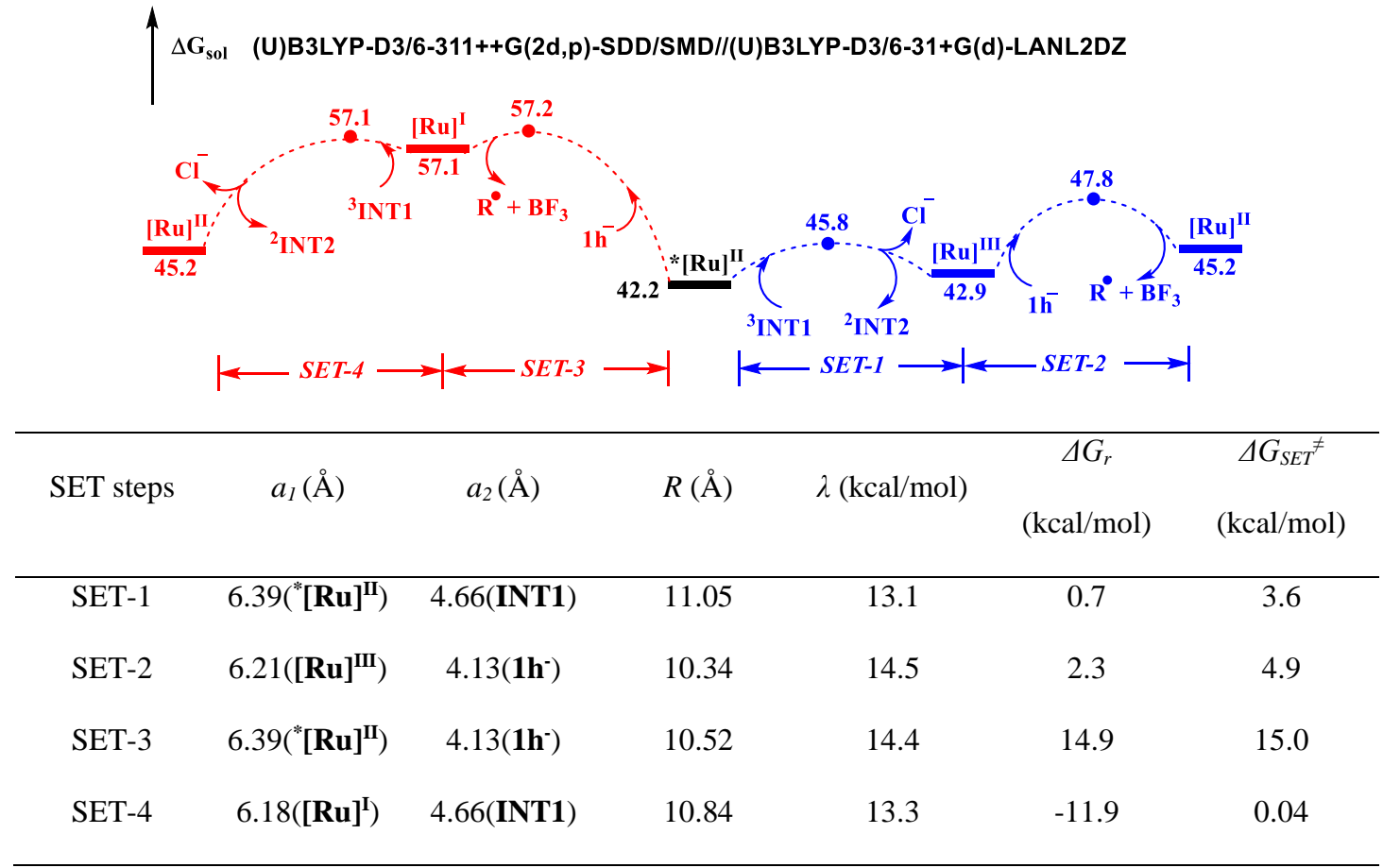




\subsection{More computational results and discussion}

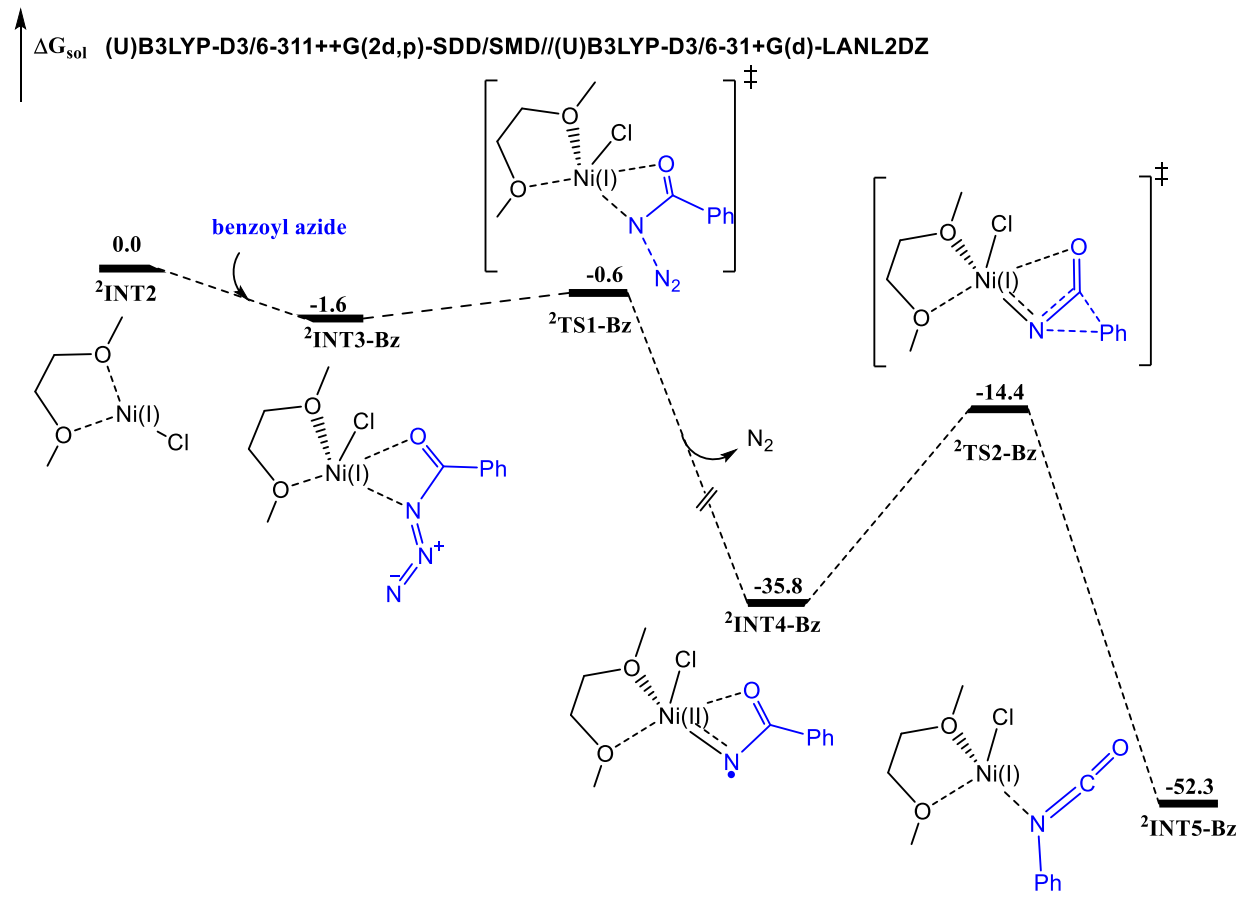

Figure S11. Energy profile (in $\mathrm{kcal} / \mathrm{mol}$ ) for the formation of Curtius rearrangement product when benzoyl azide is employed.

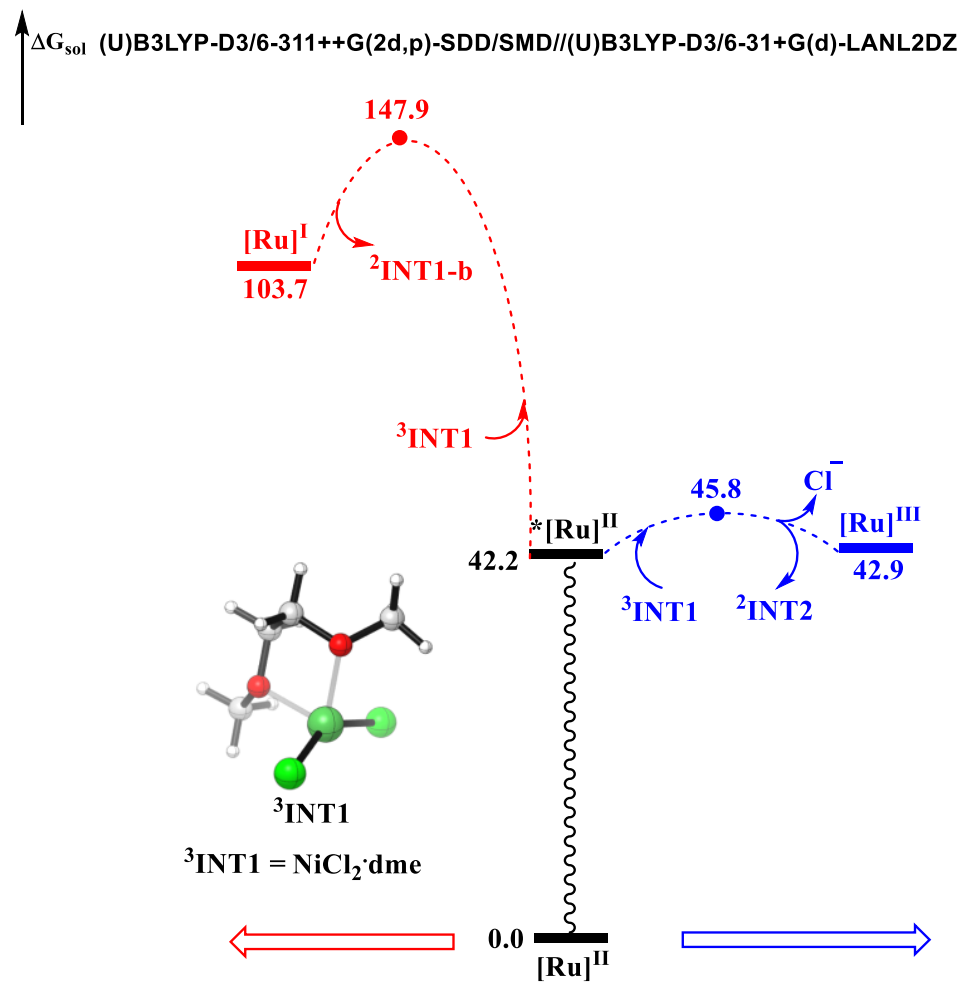

Figure S12. Comparisons of the possible quenching paths of $* \mathbf{R u}{ }^{\mathrm{II}}$ by ${ }^{3} \mathbf{I N T 1}$ (in $\mathrm{kcal} / \mathrm{mol}$ ). 

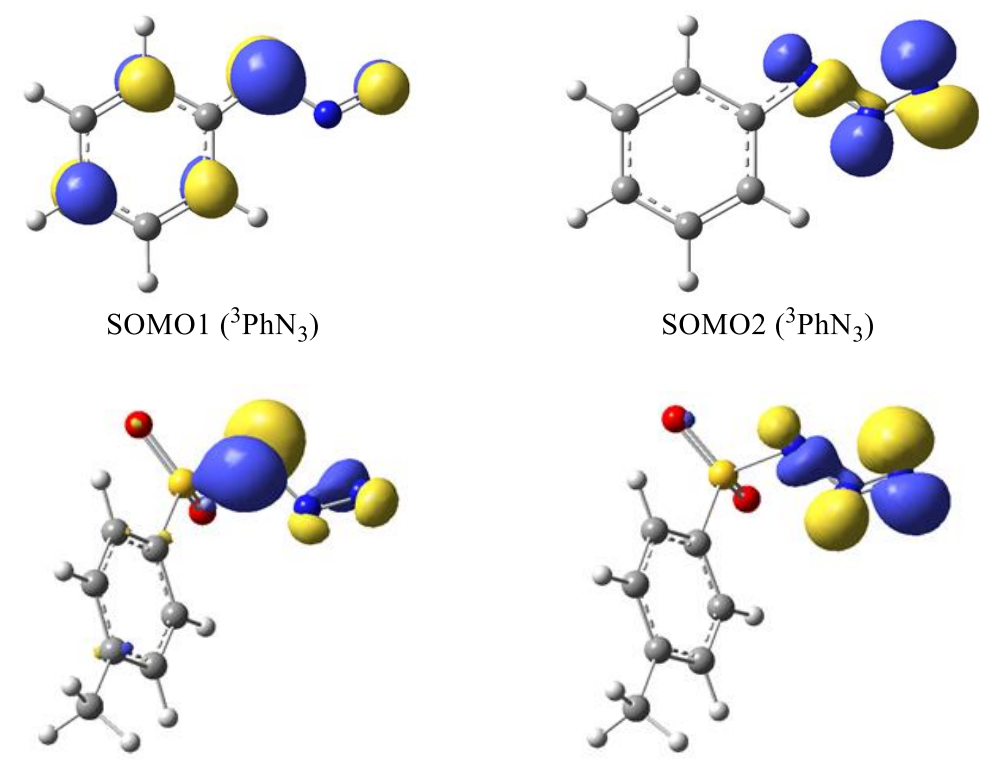

SOMO1 $\left({ }^{3} \mathrm{TsN}_{3}\right)$

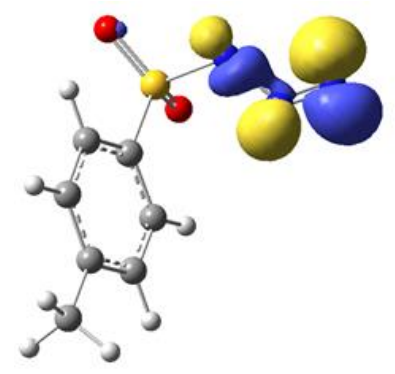

$\mathrm{SOMO} 2\left({ }^{3} \mathrm{TsN}_{3}\right)$
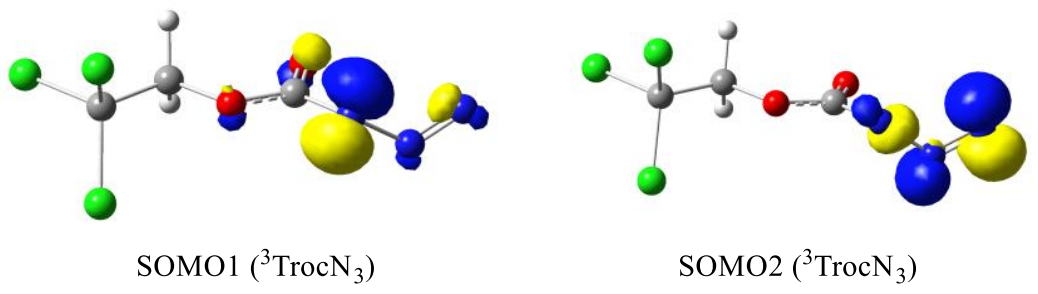

Figure S13. The two single occupied molecular orbitals (SOMO) for the triplet states of $\mathrm{PhN}_{3}, \mathrm{TsN}_{3}$ and $\operatorname{TrocN}_{3}$, respectively.

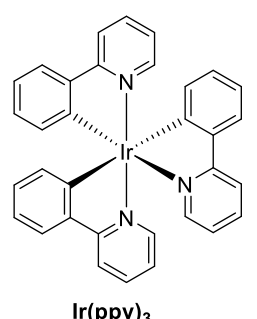

$\operatorname{lr}(\mathbf{p p y})_{3}$

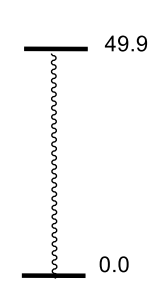

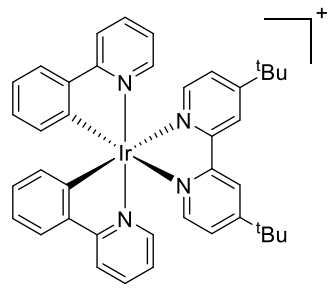

$\operatorname{Ir}(\text { ppy })_{2}($ dtbbpy $) \mathrm{PF}_{6}$

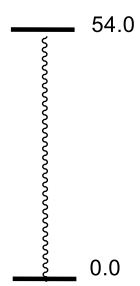

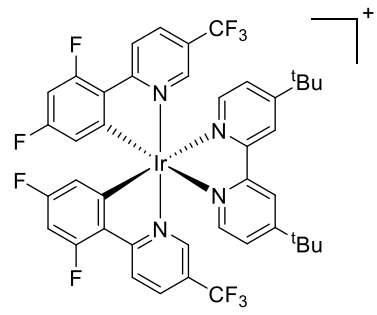

$\operatorname{Ir}\left[\mathrm{dF}\left(\mathrm{CF}_{3}\right) \mathrm{ppy}_{2}(\mathrm{dtbbpy}) \mathrm{PF}_{6}\right.$

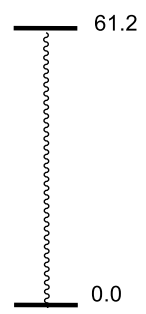

Figure S14. The calculated singlet-triplet energy gap (in kcal/mol) for the three $\mathrm{Ir}^{\mathrm{III}}$ photocatalysts. 


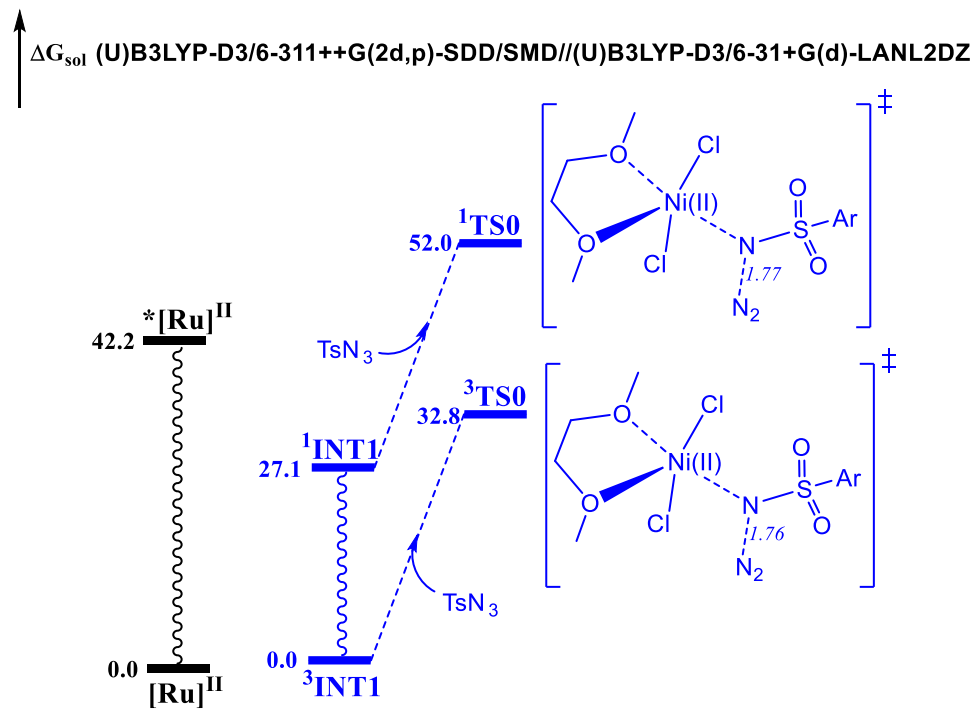

Figure S15. Comparisons of the possible energy transfer pathways (in $\mathrm{kcal} / \mathrm{mol}$ ). Bond lengths are shown in $\AA$.

One may propose that the energy transfer mechanism might occur between $* \mathrm{Ru}^{\mathrm{II}}$ and ${ }^{3} \mathbf{I N T} \mathbf{1}$. The excited singlet state ${ }^{\mathbf{1}} \mathbf{I N T} \mathbf{1}$ is calculated to be only $27.1 \mathrm{kcal} / \mathrm{mol}$ higher in energy than the ground triplet state ${ }^{3} \mathbf{I N T} \mathbf{1}$ (Figure S15). However, it is not easy for the formed ${ }^{1}$ INT1 to undergo the subsequent $\mathrm{N}_{2}$ dissociation of $\mathrm{TsN}_{3}$ at room temperature, in which the predicted $\Delta \mathrm{G}^{\neq}$is $24.9 \mathrm{kcal} / \mathrm{mol}$ relative to ${ }^{1}$ INT1. In addition, the predicted $\Delta \mathrm{G}^{\neq}$for $\mathrm{N}_{2}$ dissociation of $\mathrm{TsN}_{3}$ from ${ }^{3} \mathbf{I N T 1}$ is $33.8 \mathrm{kcal} / \mathrm{mol}$, which is also unlikely to occur at room temperature. Consequently, the energy transfer mechanism may not play an important role in this photo-promoted reaction. Overall, the SET process via the oxidative quenching pathway for the photoexcited species $* \mathrm{Ru}^{\mathrm{II}}$ to form $\mathrm{Ru}^{\mathrm{III}}$ along with the reduction of initial complex ${ }^{3} \mathrm{INT} \mathbf{1}$ to yield $\mathrm{he}^{\mathrm{N}}{ }^{\mathrm{I}}$ species ${ }^{2}$ INT2 is proposed. Subsequently, the alkyltrifluoroborate could be oxidized by $\mathrm{Ru}^{\mathrm{III}}$ via another SET to form the alkyl radical $+\mathrm{BF}_{3}$ and regenerate $\mathrm{Ru}^{\mathrm{II}}$.

After the formation of ${ }^{2} \mathbf{I N T} 2$, the yielded alkyl radical might bind with the Ni(I) species to yield ${ }^{3}$ INT3 (Figure S16). Next, 2a could coordinate with ${ }^{3}$ INT3 to form a complex ${ }^{3}$ INT4, which is ready to accept one electron from $* \mathrm{Ru}^{\mathrm{II}}$, leading to an intermediate ${ }^{4} \mathbf{I N T} \mathbf{5}^{\prime}$. It should be noted that the quartet state of INT5' is the ground state. The subsequent extrusion of $\mathrm{N}_{2}$ is explored computationally and the transition state (TS) was located as ${ }^{4} \mathrm{TS1}$, in which the N...N distance is lengthened to $1.63 \AA$. The predicted free energy barrier is only $1.3 \mathrm{kcal} / \mathrm{mol}$, indicating the dissociation of $\mathbf{N}_{2}$ for ${ }^{4} \mathbf{I N T 5}$ ' is very ready to occur. Subsequently, the formed ${ }^{4}$ INT6 would convert to its doublet ground state ${ }^{2}$ INT6, which further undergoes protonation to yield ${ }^{2} \mathbf{I N T 6}^{\prime}$. Afterward, a $\mathrm{Cl}^{-}$anion is ready to coordinate with ${ }^{\mathbf{2}} \mathbf{I N T 6}$ ' to form a stable six-coordinated complex ${ }^{2}$ INT7. From ${ }^{2}$ INT7, the reductive elimination (RE) could take place and the optimized TS is shown as ${ }^{2}$ TS2. 
The calculated activation barrier is $23.1 \mathrm{kcal} / \mathrm{mol}$ for this step, leading to the formation of ${ }^{\mathbf{2}} \mathbf{I N T 8}$. Alternatively, the RE of ${ }^{2} \mathbf{I N T 6}$ ' via ${ }^{2} \mathbf{T S 2}$ ' followed by the binding of $\mathrm{Cl}^{-}$anion can also generate ${ }^{2} \mathbf{I N T 8}$, which could not be ruled out. Finally, the desired product 3ha is produced and ${ }^{2} \mathbf{I N T} 2$ enters a next cycle.

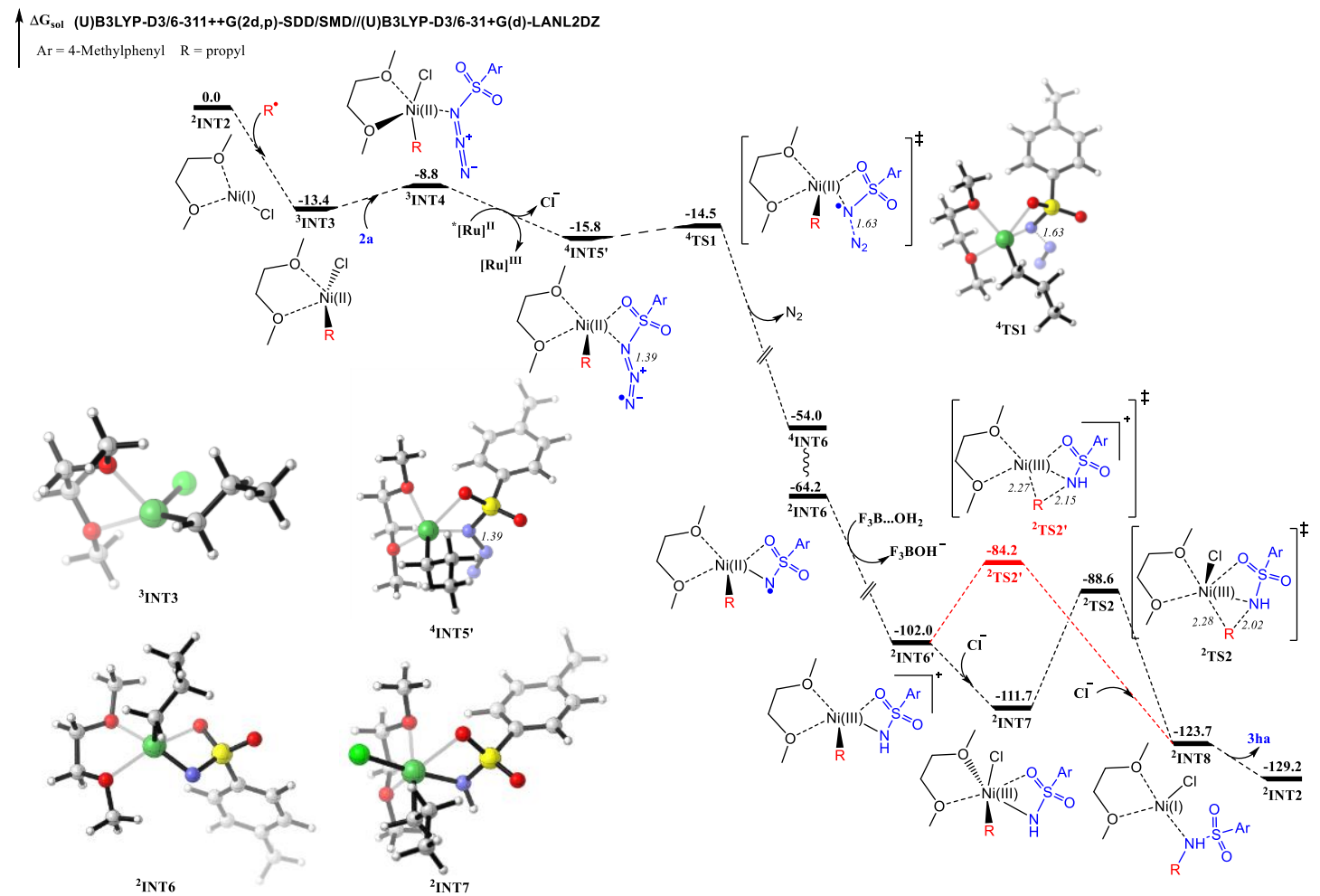

Figure S16. Energy profiles (in $\mathrm{kcal} / \mathrm{mol}$ ) for the nickel/photoredox dual catalyzed radical amidation reaction via the $\mathrm{Ni}^{\mathrm{I}} / \mathrm{Ni}^{\mathrm{II}} / \mathrm{Ni}^{\mathrm{III}} / \mathrm{Ni}^{\mathrm{I}}$ mechanistic pathway. The $3 \mathrm{D}$ structures of key intermediates and transition states are also shown. Bond lengths are shown in $\AA$.

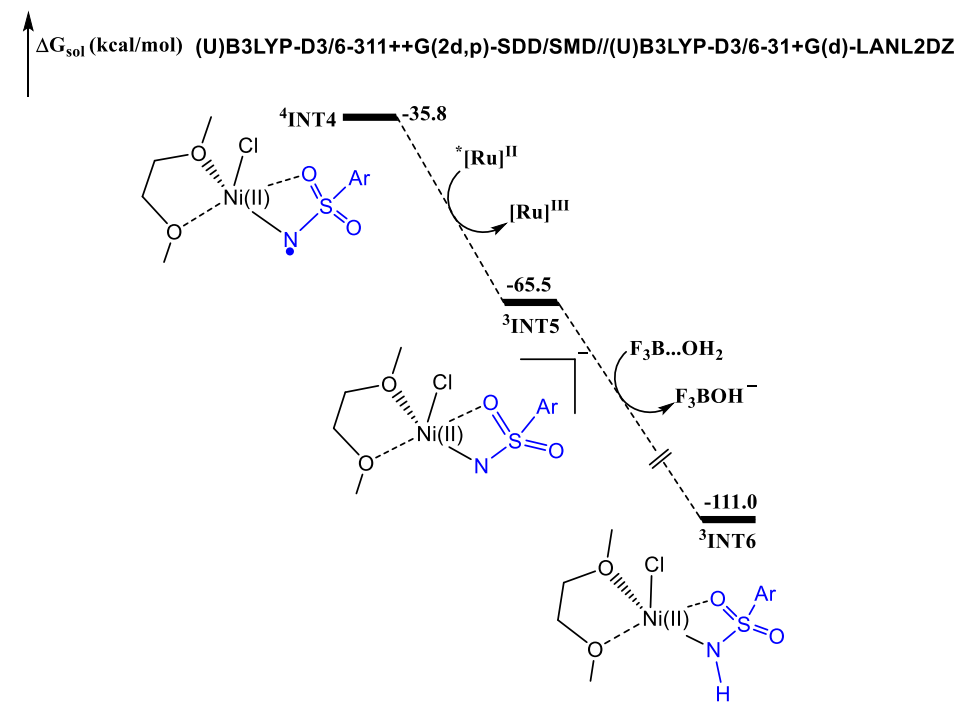

Figure S17. Energy profile (in $\mathrm{kcal} / \mathrm{mol}$ ) for the transformation from ${ }^{4} \mathbf{I N T} 4$ to ${ }^{3} \mathbf{I N T} 6$ via a stepwise process. 


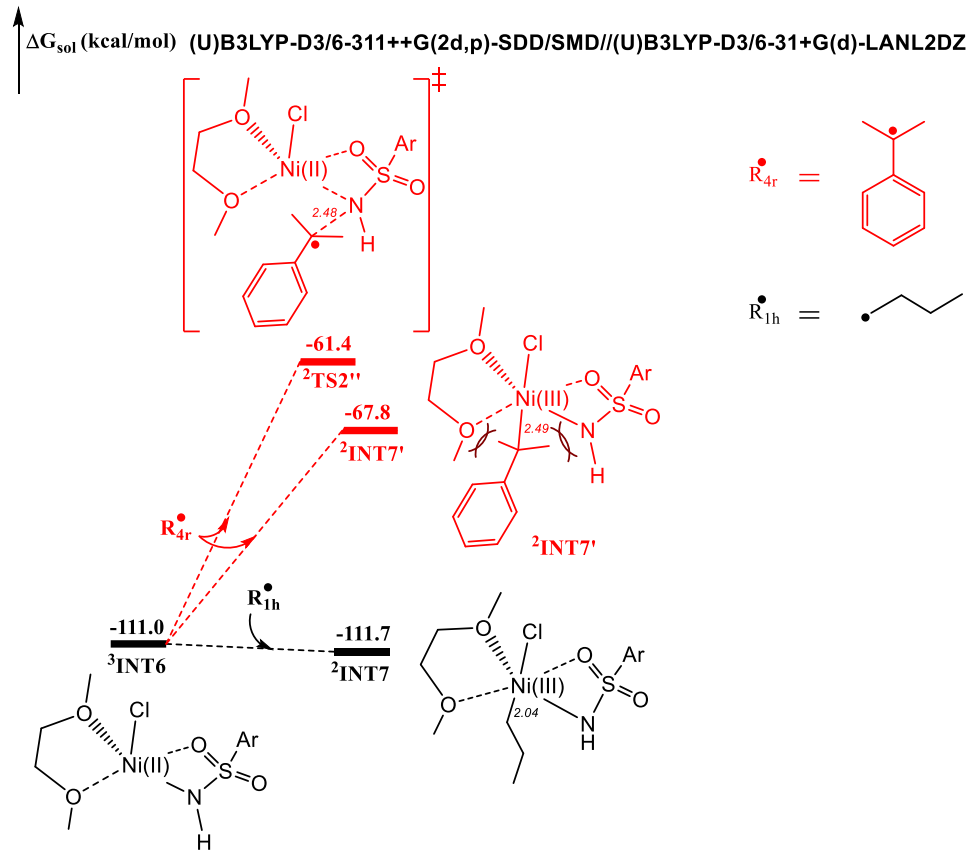

Figure S18. Energy profiles (in $\mathrm{kcal} / \mathrm{mol}$ ) for the radical (primary and tertiary radicals) addition to $\mathbf{~ N i}^{\mathrm{II}}$ center to form $\mathbf{N i}^{\mathrm{III}}$ species ${ }^{2} \mathbf{I N T 7}$ and ${ }^{2} \mathbf{I N T} 7$ '. Bond lengths are shown in $\AA$. 


\section{Preparation of Trifluoroborates}

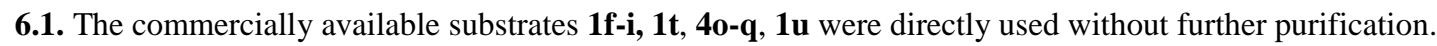

\subsection{General procedure for the preparation of $1 \mathrm{a}-\mathrm{e}, 1 \mathbf{j}, 1 \mathrm{~m}-\mathrm{p}^{9}$}

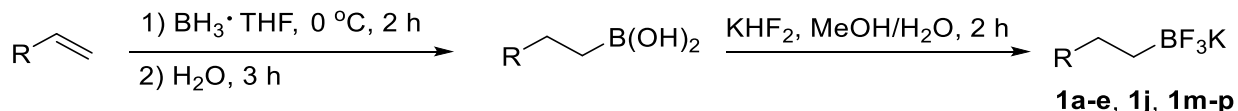

Alkene $(10.0 \mathrm{mmol})$ in THF $(2 \mathrm{~mL})$ was added dropwise to a solution of $\mathrm{BH}_{3} \cdot \mathrm{THF}(20 \mathrm{~mL}, 20 \mathrm{mmol}, 1 \mathrm{M}$ solution in THF) at $0{ }^{\circ} \mathrm{C}$. The mixture was stirred for $2 \mathrm{~h}$ at room temperature and $\mathrm{H}_{2} \mathrm{O}(2.0 \mathrm{~mL})$ was slowly added. After stirring for additional $3 \mathrm{~h}$ at room temperature, the reaction mixture was concentrated in vacuo, diluted with ethyl acetate $(30 \mathrm{~mL})$, and washed with saturated aqueous bicarbonate $(20 \mathrm{~mL})$ and brine $(20$ $\mathrm{mL}$ ). The organic layer was dried over sodium sulfate, filtered, and concentrated to near dryness, then diluted with $\mathrm{MeOH}(20 \mathrm{~mL})$ and cooled to $0{ }^{\circ} \mathrm{C}$. Saturated aqueous solution of $\mathrm{KHF}_{2}(15 \mathrm{~mL}, 3.91 \mathrm{~g}, 50$ mmol), and the resulting suspension was stirred for $2 \mathrm{~h}$ and then concentrated to dryness. The residue, a white solid, was extracted with hot acetone $(3 \times 30 \mathrm{~mL})$, and the combined filtered extracts were concentrated to approximately $5 \mathrm{~mL}$ Ether was added and the resultant precipitate was collected and dried to afford the potassium trifluoroborate as a white solid.

\subsection{General procedure for the preparation of $1 \mathrm{q}-\mathrm{s}, 4 \mathrm{l}, 4 \mathrm{~m}^{10}$}

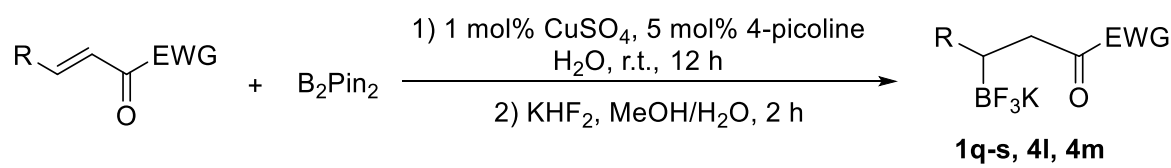

Bis(pinacolato)diboron $(2.88,11 \mathrm{mmol}), \alpha, \beta$-unsaturated carbonyl compounds (10 mmol), and 4-picoline $(50.8 \mu \mathrm{l}, 0.5 \mathrm{mmol})$ were added to a $100 \mathrm{ml}$ round-bottomed flask with a magnetic stirbar. Saturated aqueous solution of $\mathrm{CuSO}_{4}(60 \mathrm{ml}, 16 \mathrm{mg}, 0.1 \mathrm{mmol})$ was added to the reaction vessel. The reaction mixture was stirred vigorously for 1-3 hours at room temperature, typically producing a dark red or black mixture, until complete by TLC. $20 \mathrm{ml}$ EtOAc was then added and allowed to stir for 15 minutes, which produced clear or slightly colored layers. The mixture was extracted using $2 \times 30 \mathrm{ml}$ EtOAc and then washed with saturated aqueous brine $(3 \times 20 \mathrm{~mL})$. The organic layer was dried over sodium sulfate, filtered, concentrated to near dryness, then diluted with $\mathrm{MeOH}(40 \mathrm{~mL})$ and cooled to $0{ }^{\circ} \mathrm{C}$. Saturated aqueous solution of $\mathrm{KHF}_{2}(15 \mathrm{~mL}$, $3.91 \mathrm{~g}, 50 \mathrm{mmol}$ ), and the resulting suspension was stirred for $2 \mathrm{~h}$ and then concentrated to dryness. The residue, a white solid, was extracted with hot acetone $(3 \times 30 \mathrm{~mL})$, and the combined filtered extracts were 
concentrated to approximately $5 \mathrm{~mL}$ Ether was added and the resultant precipitate was collected and dried to afford the potassium trifluoroborate as a white solid.

\subsection{General procedure for the preparation of $1 \mathrm{k}-\mathrm{l}, 4 \mathrm{a}-\mathrm{c}, 4 \mathrm{e}, 4 \mathrm{~g}-\mathrm{k}^{11}$}

$$
\begin{aligned}
& \text { 1) } 10 \mathrm{~mol} \% \mathrm{Cul}, 13 \mathrm{~mol} \% \mathrm{PPh}_{3} \\
& \text { Alkyl- } \mathrm{Br}+\mathrm{B}_{2} \mathrm{Pin}_{2} \frac{\mathrm{LiOMe}, \mathrm{DMF}, 25^{\circ} \mathrm{C}, 18 \mathrm{~h}}{2) \mathrm{KHF}_{2}, \mathrm{MeOH} / \mathrm{H}_{2} \mathrm{O}, 2 \mathrm{~h}} \begin{array}{c}
\text { Alkyl- } \mathrm{BF}_{3} \mathrm{~K} \\
\text { 1k-l, 4a-c, 4e, 4g-k }
\end{array}
\end{aligned}
$$

$\mathrm{CuI}(190 \mathrm{mg}, 1.0 \mathrm{mmol}), \mathrm{PPh}_{3}(341 \mathrm{mg}, 1.3 \mathrm{mmol}), \mathrm{LiOMe}(760 \mathrm{mg}, 20.0 \mathrm{mmol})$, and bis(pinacolato)diboron $(3.80 \mathrm{~g}, 15.0 \mathrm{mmol})$ were added to a $100 \mathrm{~mL}$ round-bottomed flask equipped with a stir bar. The vessel was evacuated and filled with nitrogen gas three times. DMF (40 mL) and the alkyl bromide $(10.0 \mathrm{mmol})$ were added by syringe under a nitrogen atmosphere. The resulting reaction mixture was stirred vigorously at $25^{\circ} \mathrm{C}$ for $18 \mathrm{~h}$. The reaction mixture was diluted with EtOAc and filtered through silica gel. Then the mixture was washed with saturated aqueous brine $(3 \times 100 \mathrm{~mL})$. The organic layer was dried over sodium sulfate, filtered, concentrated to near dryness, then diluted with $\mathrm{MeOH}(40 \mathrm{~mL})$ and cooled to $0{ }^{\circ} \mathrm{C}$. Saturated aqueous solution of $\mathrm{KHF}_{2}(15 \mathrm{~mL}, 3.91 \mathrm{~g}, 50 \mathrm{mmol})$, and the resulting suspension was stirred for $2 \mathrm{~h}$ and then concentrated to dryness. The residue, a white solid, was extracted with hot acetone $(3 \times 30 \mathrm{~mL})$, and the combined filtered extracts were concentrated to approximately $5 \mathrm{~mL}$ Ether was added and the resultant precipitate was collected and dried to afford the potassium trifluoroborate as a white solid.

\subsection{General procedure for the preparation of $4 d, 4 f^{12}$}

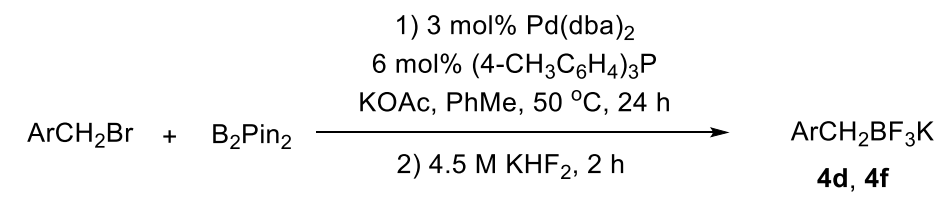

To a solution of alkyl bromide $(5.0 \mathrm{mmol})$ in toluene $(30 \mathrm{~mL})$ at room temperature under argon atmosphere was added KOAc (736 mg, $7.5 \mathrm{mmol}),\left(4-\mathrm{MeC}_{6} \mathrm{H}_{4}\right)_{3} \mathrm{P}(91 \mathrm{mg}, 0.3 \mathrm{mmol}), \mathrm{Pd}(\mathrm{dba})_{2}(86 \mathrm{mg}, 0.15$ mmol) and bis(pinacolato)diboron $(1.40 \mathrm{~g}, 5.5 \mathrm{mmol})$. The mixture was stirred vigorously under argon atmosphere for $24 \mathrm{~h}$. The reaction mixture was diluted with EtOAc and filtered through silica gel. The resultant yellow-brown mixture was concentrated to near dryness, then diluted with $\mathrm{MeOH}(20 \mathrm{~mL})$ and cooled to $0{ }^{\circ} \mathrm{C}$. Saturated aqueous solution of $\mathrm{KHF}_{2}(9.0 \mathrm{~mL}, 40.5 \mathrm{mmol})$ was added dropwise over $10 \mathrm{~min}$. and the resulting suspension was stirred for $2 \mathrm{~h}$ and then concentrated to dryness. The residue, a white solid, was extracted with hot acetone $(3 \times 30 \mathrm{~mL})$, and the combined filtered extracts were concentrated to 
approximately $5 \mathrm{~mL}$ Ether was added and the resultant precipitate was collected and dried to afford the potassium trifluoroborate as a white solid.

\subsection{General procedure for the preparation of $4 r^{13}$}

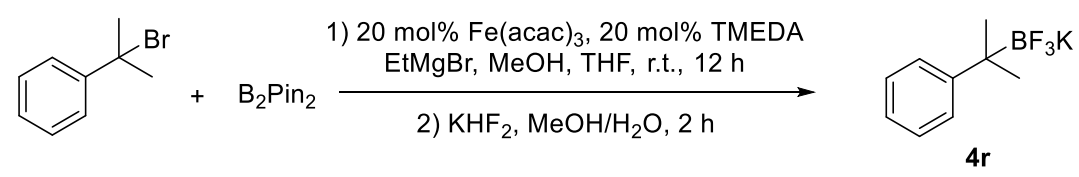

$\mathrm{Fe}(\mathrm{acac})_{3}(706,2.0 \mathrm{mmol})$ and bis(pinacolato)diboron $(8.9 \mathrm{~g}, 35.0 \mathrm{mmol})$ were added to a $100 \mathrm{~mL}$ roundbottomed flask equipped with a stir bar. The vessel was evacuated and filled with nitrogen gas three times. THF $(50 \mathrm{~mL})$ was added, followed by TMEDA $(8 \mathrm{~mL}$ of a $4 \%(\mathrm{v} / \mathrm{v})$ solution of TMEDA $(0.32 \mathrm{~mL})$ in THF, $2.0 \mathrm{mmol})$. Next, ethylmagnesium bromide $\left(15 \mathrm{~mL}\left(3 \mathrm{M}\right.\right.$ in $\left.\left.\mathrm{Et}_{2} \mathrm{O}\right), 45.0 \mathrm{mmol}\right)$ was added dropwise to the reaction mixture at a rate of one drop/sec. Finally, 1-bromoadamantane $(10.0 \mathrm{mmol})$ was added to the reaction mixture at once. The reaction mixture was stirred at room temperature for $12 \mathrm{~h}$. Mixture was filtered (washed with $\mathrm{Et}_{2} \mathrm{O}$ ) and concentrated to near dryness, then diluted with $\mathrm{MeOH}(40 \mathrm{~mL})$ and cooled to $0{ }^{\circ} \mathrm{C}$, Saturated aqueous solution of $\mathrm{KHF}_{2}(15 \mathrm{~mL}, 3.91 \mathrm{~g}, 50 \mathrm{mmol})$, and the resulting suspension was stirred for 2 $\mathrm{h}$ and then concentrated to dryness. The residue, a white solid, was extracted with hot acetone $(3 \times 30 \mathrm{~mL})$, and the combined filtered extracts were concentrated to approximately $5 \mathrm{~mL}$ Ether was added and the resultant precipitate was collected and dried to afford the potassium trifluoroborate as a white solid.

\subsection{General procedure for the preparation of sulfonyl azides $2 b-\mathbf{l}^{14}$}

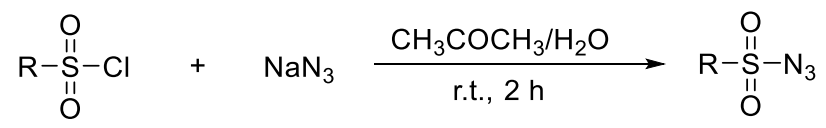

To a solution of sulfonyl chloride $(10 \mathrm{mmol})$ in acetone at room temperature was added a solution of $\mathrm{NaN}_{3}$ $(1.3 \mathrm{~g}, 20 \mathrm{mmol})$ in water $(10 \mathrm{~mL})$. The mixture was stirred for $2 \mathrm{~h}$ at room temperature. The mixture was concentrated, diluted with water, and extracted with $\mathrm{CH}_{2} \mathrm{Cl}_{2}(2 \times 30 \mathrm{~mL})$. The combined organic extracts were dried over sodium sulfate, filtered, and concentrated. The residue was purified by flash chromatography.

\subsection{General procedure for the preparation of azidoformates $6 a^{-} d^{15}$}

To a well-stirred suspension solution of sodium azide $(1.6 \mathrm{~g}, 25 \mathrm{mmol})$ in acetone $(30 \mathrm{ml})$ in a $50 \mathrm{~mL}$ round bottom flask with a magnetic bar, carbonochloridate $(20 \mathrm{mmol})$ was added dropwise for 10 minutes at room temperature. Then the flask was sealed, under the condition of protection from light, stirred under 
room temperature overnight. The solution was filtered by diatomite and washed with ethyl acetate, then the filtrate was concentrated in vacuo and purified via chromatography column (petroleum ether/ethyl acetate = 10:1) to provide the azidoformates.

\subsection{General procedure for the preparation of t-Butyl azidoformates $\left(\mathrm{BocN}_{3}\right) 6 \mathrm{e}^{16}$}

t-Butyl carbazate $(3.00 \mathrm{~g}, 22.7 \mathrm{mmol}, 1.0$ eq.) was dissolved in a mixture of water $(7.0 \mathrm{~mL})$ and acetic acid (5.0 mL) and cooled to $0{ }^{\circ} \mathrm{C}$ Over 5 minutes. $\mathrm{NaNO}_{2}(1.72 \mathrm{~g}, 25.0 \mathrm{mmol}, 1.1$ eq.) was added portion-wise. The reaction mixture was stirred for two hours and warmed to room temperature. Water $(20 \mathrm{~mL})$ was added and the mixture was extracted with $\mathrm{Et}_{2} \mathrm{O}(3 \times 70 \mathrm{~mL})$. The organic layer was washed with half saturated $\mathrm{NaHCO}_{3}(2 \times 50 \mathrm{~mL})$, and brine $(30 \mathrm{~mL})$, dried over $\mathrm{Na}_{2} \mathrm{SO}_{4}$ and filtered. The solvent was removed in vacuo at room temperature. The $\mathrm{BocN}_{3}(3.25 \mathrm{~g}, 22.7 \mathrm{mmol}$, quant.) was obtained as a yellow liquid and was pure enough for further applications.

\section{Synthesis and characterization of products}

\subsection{Typical procedure for the radical amidation reactions.}

Typical procedure for the cross-coupling reactions of alkyltrifluoroborates ( 1 or 4 ) and sulfonyl azides (2). To a dried sealed tube $(10 \mathrm{~mL})$ equipped with a magnetic stir bar were added successively $\mathrm{Ru}(\mathrm{bpy})_{3}\left(\mathrm{PF}_{6}\right)_{2}(17.2 \mathrm{mg}, 0.02 \mathrm{mmol}), \mathrm{NiCl}_{2} \cdot \mathrm{dme}(8.8 \mathrm{mg}, 0.04 \mathrm{mmol})$, alkyltrifluoroborates 1 or $4(0.20$ mmol) under the atmosphere of argon. Next, sulfonyl azides $2(0.40 \mathrm{mmol})$ and $\mathrm{CH}_{3} \mathrm{CN}(2.0 \mathrm{~mL})$ were added into the tube. The mixture was stirred and irradiated with $3 \mathrm{~W} \times 2$ purple LEDs at room temperature for $24 \mathrm{~h}$. After the removal of solvent, the residue was purified by column chromatography on silica gel with a gradient eluent of petroleum ether and ethyl acetate to provide the pure product 3 .

Typical procedure for the cross-coupling reactions of alkyltrifluoroborates 1 and azidoformates 6 . To a dried sealed tube $(10 \mathrm{~mL})$ equipped with a magnetic stir bar were added successively $\mathrm{Ru}(\mathrm{bpy})_{3}\left(\mathrm{PF}_{6}\right)_{2}(4.3$ mg, $0.005 \mathrm{mmol}), \mathrm{NiCl}_{2} \cdot \mathrm{dme}(4.4 \mathrm{mg}, 0.02 \mathrm{mmol})$, alkyltrifluoroborates $1(0.20 \mathrm{mmol})$ under the atmosphere of argon. Next, azidoformates $6(0.60 \mathrm{mmol})$ and $\mathrm{CH}_{3} \mathrm{CN}(2.0 \mathrm{~mL})$ were added into the tube. The mixture was stirred and irradiated with $3 \mathrm{~W} \times 2$ blue LEDs at room temperature for $24 \mathrm{~h}$. After the removal of solvent, the residue was purified by column chromatography on silica gel with a gradient eluent of petroleum ether and ethyl acetate to provide the pure product 7 . 


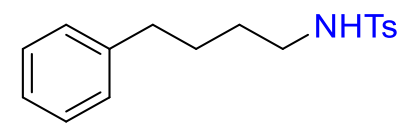

\section{4-Methyl- $N$-(4-phenylbutyl)benzenesulfonamide (3aa)}

White solid, m.p.: $50.6-51.0{ }^{\circ} \mathrm{C} ;{ }^{1} \mathrm{H}$ NMR $\left(400 \mathrm{MHz}, \mathrm{CDCl}_{3}\right) \delta 7.73(\mathrm{~d}, J=8.1 \mathrm{~Hz}, 2 \mathrm{H}), 7.28(\mathrm{~d}, J=8.1 \mathrm{~Hz}$, 2H), $7.26-7.21(\mathrm{~m}, 2 \mathrm{H}), 7.20-7.13(\mathrm{~m}, 1 \mathrm{H}), 7.11-7.06(\mathrm{~m}, 2 \mathrm{H}), 4.59(\mathrm{t}, J=6.2 \mathrm{~Hz}, 1 \mathrm{H}), 2.94(\mathrm{q}, J=6.9$ $\mathrm{Hz}, 2 \mathrm{H}), 2.54(\mathrm{t}, J=7.5 \mathrm{~Hz}, 2 \mathrm{H}), 2.41(\mathrm{~s}, 3 \mathrm{H}), 1.63-1.54(\mathrm{~m}, 2 \mathrm{H}), 1.52-1.43(\mathrm{~m}, 2 \mathrm{H}) ;{ }^{13} \mathrm{C}$ NMR $(101$ $\left.\mathrm{MHz}, \mathrm{CDCl}_{3}\right) \delta 143.38,141.91,137.02,129.76,128.41,128.37,127.15,125.87,43.08,35.29,29.11,28.26$, 21.58; IR (neat): $v\left(\mathrm{~cm}^{-1}\right)$ 3284, 3029, 2945, 2923, 2865, 1597, 1304, 1155, 815; HRMS (ESI+) m/z calcd. $\mathrm{C}_{17} \mathrm{H}_{22} \mathrm{NO}_{2} \mathrm{~S}^{+}[\mathrm{M}+\mathrm{H}]^{+}:$304.1366, found: 304.1362 .<smiles>Cc1ccc(CC[NH2+][Na])cc1</smiles>

\section{4-methyl- $N$-(4-methylphenethyl)benzenesulfonamide (3ba)}

Yellow oil; ${ }^{1} \mathrm{H}$ NMR $\left(400 \mathrm{MHz}, \mathrm{CDCl}_{3}\right) \delta 7.69(\mathrm{~d}, J=8.1 \mathrm{~Hz}, 2 \mathrm{H}), 7.28(\mathrm{~d}, J=8.1 \mathrm{~Hz}, 2 \mathrm{H}), 7.07(\mathrm{~d}, J=7.8$ Hz, 2H), 6.96 (d, J=7.8 Hz, 2H), 4.40 (brs, 1H), 3.18 (q, $J=6.8 \mathrm{~Hz}, 2 \mathrm{H}), 2.71(\mathrm{t}, J=7.0 \mathrm{~Hz}, 2 \mathrm{H}), 2.42$ (s, 3H), $2.31(\mathrm{~s}, 3 \mathrm{H}) ;{ }^{13} \mathrm{C} \mathrm{NMR}\left(101 \mathrm{MHz}, \mathrm{CDCl}_{3}\right) \delta 143.51,137.05,136.52,134.62,129.81,129.55,128.73$, 127.23, 44.40, 35.42, 21.65, 21.14; IR (neat): $v\left(\mathrm{~cm}^{-1}\right)$ 3278, 2970, 2924, 2870, 1598, 1322, 1155, 660; HRMS (ESI+) m/z calcd. $\mathrm{C}_{16} \mathrm{H}_{19} \mathrm{NNaO}_{2} \mathrm{~S}^{+}[\mathrm{M}+\mathrm{Na}]^{+}: 312.1029$, found: 312.1031 .<smiles>[SH3+][NH2+]CC(c1ccccc1)c1ccccc1</smiles>

$N$-(2,2-diphenylethyl)-4-methylbenzenesulfonamide (3ca)

White solid, m.p.: $124.0-124.8{ }^{\circ} \mathrm{C} ;{ }^{1} \mathrm{H}$ NMR $\left(400 \mathrm{MHz}, \mathrm{CDCl}_{3}\right) \delta 7.68(\mathrm{~d}, J=8.4 \mathrm{~Hz}, 2 \mathrm{H}), 7.30(\mathrm{~d}, J=8.4$ $\mathrm{Hz}, 2 \mathrm{H}), 7.29-7.24(\mathrm{~m}, 4 \mathrm{H}), 7.23-7.18(\mathrm{~m}, 2 \mathrm{H}), 7.13-7.05(\mathrm{~m}, 4 \mathrm{H}), 4.32(\mathrm{t}, J=6.2 \mathrm{~Hz}, 1 \mathrm{H}), 4.06(\mathrm{t}, J=$ $7.9 \mathrm{~Hz}, 1 \mathrm{H}), 3.55(\mathrm{dd}, J=7.9,6.2 \mathrm{~Hz}, 2 \mathrm{H}), 2.44(\mathrm{~s}, 3 \mathrm{H}) ;{ }^{13} \mathrm{C} \mathrm{NMR}\left(101 \mathrm{MHz}, \mathrm{CDCl}_{3}\right) \delta 143.69,140.83$, 136.92, 129.90, 129.02, 128.06, 127.29, 50.70, 47.39, 21.70; IR (neat): $v\left(\mathrm{~cm}^{-1}\right) 3301,2987,2921,1597$, 1327, 1160, 1066, 660; HRMS (ESI+) m/z calcd. $\mathrm{C}_{21} \mathrm{H}_{21} \mathrm{NNaO}_{2} \mathrm{~S}^{+}[\mathrm{M}+\mathrm{Na}]^{+}:$374.1185, found: 374.1182 .<smiles>[AsH3]NCCCc1ccccc1</smiles> 
Pale yellow oil; ${ }^{1} \mathrm{H}$ NMR $\left(400 \mathrm{MHz}, \mathrm{CDCl}_{3}\right) \delta 7.74(\mathrm{~d}, J=8.1 \mathrm{~Hz}, 2 \mathrm{H}), 7.29(\mathrm{~d}, J=8.1 \mathrm{~Hz}, 2 \mathrm{H}), 7.26-7.21$ (m, 2H), $7.19-7.13(\mathrm{~m}, 1 \mathrm{H}), 7.07(\mathrm{~d}, J=7.6 \mathrm{~Hz}, 2 \mathrm{H}), 4.92(\mathrm{t}, J=6.1 \mathrm{~Hz}, 1 \mathrm{H}), 2.94(\mathrm{q}, J=6.6 \mathrm{~Hz}, 2 \mathrm{H})$, $2.58(\mathrm{t}, J=7.7 \mathrm{~Hz}, 2 \mathrm{H}), 2.42(\mathrm{~s}, 3 \mathrm{H}), 1.85-1.62(\mathrm{~m}, 2 \mathrm{H}) ;{ }^{13} \mathrm{C} \mathrm{NMR}\left(101 \mathrm{MHz}, \mathrm{CDCl}_{3}\right) \delta 143.47,141.06$, 137.03, 129.82, 128.53, 128.46, 127.21, 126.13, 42.72, 32.80, 31.22, 21.61; IR (neat): $v\left(\mathrm{~cm}^{-1}\right) 3278,2925$, 2864, 1599, 1321, 1154, 1092, 660; HRMS (ESI+) m/z calcd. $\mathrm{C}_{16} \mathrm{H}_{20} \mathrm{NO}_{2} \mathrm{~S}^{+}[\mathrm{M}+\mathrm{H}]^{+}:$290.1209, found: 290.1207.<smiles>[SnH3]CCCCCc1ccccc1</smiles>

\section{4-Methyl- $N$-(5-phenylpentyl)benzenesulfonamide (3ea)}

Yellow oil; ${ }^{1} \mathrm{H}$ NMR $\left(400 \mathrm{MHz}, \mathrm{CDCl}_{3}\right) \delta 7.66(\mathrm{~d}, J=8.1 \mathrm{~Hz}, 2 \mathrm{H}), 7.23(\mathrm{~d}, J=8.1 \mathrm{~Hz}, 2 \mathrm{H}), 7.20-7.16(\mathrm{~m}$, 2H), $7.12-7.07(\mathrm{~m}, 1 \mathrm{H}), 7.08-7.01(\mathrm{~m}, 2 \mathrm{H}), 4.37(\mathrm{t}, J=6.2 \mathrm{~Hz}, 1 \mathrm{H}), 2.85(\mathrm{q}, J=6.8 \mathrm{~Hz}, 2 \mathrm{H}), 2.56-2.43$ (m, 2H), $2.35(\mathrm{~s}, 3 \mathrm{H}), 1.51-1.45(\mathrm{~m}, 2 \mathrm{H}), 1.46-1.34(\mathrm{~m}, 2 \mathrm{H}), 1.28-1.13(\mathrm{~m}, 2 \mathrm{H}) ;{ }^{13} \mathrm{C} \mathrm{NMR}(101 \mathrm{MHz}$, $\left.\mathrm{CDCl}_{3}\right) \delta 143.49,142.37,137.12,129.83,128.47,128.43,127.24,125.88,43.24,35.81,30.95,29.60,26.23$, 21.65; IR (neat): $v\left(\mathrm{~cm}^{-1}\right) 3279,2928,2856,1322,1154,660$; HRMS (ESI+) m/z calcd. $\mathrm{C}_{18} \mathrm{H}_{24} \mathrm{NO}_{2} \mathrm{~S}^{+}$ $[\mathrm{M}+\mathrm{H}]^{+}:$318.1522, found: 318.1528 .

- NHTs

$N$, 4-Dimethylbenzenesulfonamide (3fa)

Colorless oil; ${ }^{1} \mathrm{H}$ NMR $\left(400 \mathrm{MHz}, \mathrm{CDCl}_{3}\right) \delta 7.75(\mathrm{~d}, J=7.9 \mathrm{~Hz}, 2 \mathrm{H}), 7.33(\mathrm{~d}, J=7.9 \mathrm{~Hz}, 2 \mathrm{H}), 4.41$ (brs, $\left.1 \mathrm{H}\right)$, $2.65(\mathrm{~d}, J=5.4 \mathrm{~Hz}, 3 \mathrm{H}), 2.44(\mathrm{~s}, 3 \mathrm{H}) ;{ }^{13} \mathrm{C} \mathrm{NMR}\left(101 \mathrm{MHz}, \mathrm{CDCl}_{3}\right) \delta 143.67,135.93,129.87,127.41,29.47$, 21.66; IR (neat): $v\left(\mathrm{~cm}^{-1}\right) 3270,2926,1596,1316,1154,1089,661$; HRM (ESI+) m/z calcd. $\mathrm{C}_{8} \mathrm{H}_{12} \mathrm{NO}_{2} \mathrm{~S}^{+}$ $[\mathrm{M}+\mathrm{H}]^{+}:$186.0583, found: 186.0578

$$
\widehat{\mathrm{NHTS}}
$$

\section{$N$-ethyl-4-methylbenzenesulfonamide (3ga)}

White solid, m.p.: $104.6-105.1{ }^{\circ} \mathrm{C} ;{ }^{1} \mathrm{H}$ NMR $\left(400 \mathrm{MHz}, \mathrm{CDCl}_{3}\right) \delta 7.75(\mathrm{~d}, J=7.9 \mathrm{~Hz}, 2 \mathrm{H}), 7.31(\mathrm{~d}, J=7.9$ $\mathrm{Hz}, 2 \mathrm{H}), 4.38$ (brs, 1H), $3.04-2.97$ (m, 2H), 2.43 (s, 3H), $1.10(\mathrm{t}, J=7.3 \mathrm{~Hz}, 3 \mathrm{H}) ;{ }^{13} \mathrm{C} \mathrm{NMR}(101 \mathrm{MHz}$, $\left.\mathrm{CDCl}_{3}\right) \delta 143.53,137.13,129.84,127.26,38.37,21.65,15.23$; IR (neat): $v\left(\mathrm{~cm}^{-1}\right) 3267,2918,1764,1321$, 1158, 1058, 739, 698, 660; HRMS (ESI+) m/z calcd. $\mathrm{C}_{9} \mathrm{H}_{14} \mathrm{NO}_{2} \mathrm{~S}^{+}[\mathrm{M}+\mathrm{H}]^{+}:$: 200.0740, found: 200.0744. 
$\widehat{N H T s}$

4-Methyl- $N$-propylbenzenesulfonamide (3ha)

Yellow oil; ${ }^{1} \mathrm{H}$ NMR $\left(400 \mathrm{MHz}, \mathrm{CDCl}_{3}\right) \delta 7.75(\mathrm{~d}, J=8.3 \mathrm{~Hz}, 2 \mathrm{H}), 7.30(\mathrm{~d}, J=8.3 \mathrm{~Hz}, 2 \mathrm{H}), 4.49(\mathrm{t}, J=6.3$ $\mathrm{Hz}, 1 \mathrm{H}), 2.90(\mathrm{q}, J=7.0 \mathrm{~Hz}, 2 \mathrm{H}), 2.42(\mathrm{~s}, 3 \mathrm{H}), 1.54-1.39(\mathrm{~m}, 2 \mathrm{H}), 0.86(\mathrm{t}, J=7.4 \mathrm{~Hz}, 3 \mathrm{H}) ;{ }^{13} \mathrm{C}$ NMR $(101$ $\left.\mathrm{MHz}, \mathrm{CDCl}_{3}\right) \delta 143.47,137.18,129.82,127.23,45.10,23.07,21.65,11.23$; IR (neat): $v\left(\mathrm{~cm}^{-1}\right) 3280,2966$, 2934, 2876, 1156, 660; HRMS (ESI+) m/z calcd. $\mathrm{C}_{10} \mathrm{H}_{16} \mathrm{NO}_{2} \mathrm{~S}^{+}[\mathrm{M}+\mathrm{H}]^{+}:$214.0896, found: 214.0890 .

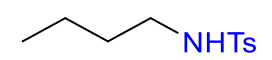

$N$-Butyl-4-methylbenzenesulfonamide (3ia)

Pale yellow oil; ${ }^{1} \mathrm{H}$ NMR $\left(400 \mathrm{MHz}, \mathrm{CDCl}_{3}\right) \delta 7.76(\mathrm{~d}, J=7.9 \mathrm{~Hz}, 2 \mathrm{H}), 7.31(\mathrm{~d}, J=7.9 \mathrm{~Hz}, 2 \mathrm{H}), 4.74(\mathrm{t}, J=$ $6.2 \mathrm{~Hz}, 1 \mathrm{H}), 2.92(\mathrm{q}, J=6.8 \mathrm{~Hz}, 2 \mathrm{H}), 2.43(\mathrm{~s}, 3 \mathrm{H}) ; 1.47-1.40(\mathrm{~m}, 2 \mathrm{H}), 1.33-1.24(\mathrm{~m}, 2 \mathrm{H}), 0.84(\mathrm{t}, J=7.3$ $\mathrm{Hz}, 3 \mathrm{H}) ;{ }^{13} \mathrm{C} \mathrm{NMR}\left(101 \mathrm{MHz}, \mathrm{CDCl}_{3}\right) \delta 143.37,137.07,129.75,127.19,42.99,31.62,21.59,19.77,13.61$; IR (neat): $v\left(\mathrm{~cm}^{-1}\right)$ 3280, 2960, 2932, 2873, 1599, 1321, 1156, 660; HRMS (ESI+) m/z calcd. $\mathrm{C}_{11} \mathrm{H}_{18} \mathrm{NO}_{2} \mathrm{~S}^{+}$ $[\mathrm{M}+\mathrm{H}]^{+}:$228.1053, found: 228.1058 .

TMS $\sim \mathrm{NHTS}$

4-Methyl- $N$-(3-(trimethylsilyl)propyl)benzenesulfonamide (3ja)

Pale yellow oil; ${ }^{1} \mathrm{H}$ NMR $\left(400 \mathrm{MHz}, \mathrm{CDCl}_{3}\right) \delta 7.75(\mathrm{~d}, J=7.9 \mathrm{~Hz}, 2 \mathrm{H}), 7.30(\mathrm{~d}, J=7.9 \mathrm{~Hz}, 2 \mathrm{H}), 4.60(\mathrm{t}, J=$ $6.3 \mathrm{~Hz}, 1 \mathrm{H}), 2.91(\mathrm{q}, J=6.8 \mathrm{~Hz}, 2 \mathrm{H}), 2.42(\mathrm{~s}, 3 \mathrm{H}), 1.45-1.32(\mathrm{~m}, 2 \mathrm{H}), 0.43-0.34(\mathrm{~m}, 2 \mathrm{H}),-0.08(\mathrm{~s}, 9 \mathrm{H})$; ${ }^{13} \mathrm{C} \mathrm{NMR}\left(101 \mathrm{MHz}, \mathrm{CDCl}_{3}\right) \delta 143.42,137.33,129.79,127.21,46.41,24.40,21.62,13.63,-1.76$; IR (neat): $v\left(\mathrm{~cm}^{-1}\right)$ 3280, 2952, 2876, 1599, 1323, 1155, 835, 660; HRMS (ESI+) m/z calcd. $\mathrm{C}_{13} \mathrm{H}_{24} \mathrm{NO}_{2} \mathrm{SSi}^{+}[\mathrm{M}+\mathrm{H}]^{+}:$ 286.1292, found: 286.1296 .

$\mathrm{Ph} \sim \mathrm{NHTs}^{\sim}$

4-Methyl- $N$-(2-phenoxyethyl)benzenesulfonamide (3ka)

White solid, m.p.: $100.9-101.8{ }^{\circ} \mathrm{C} ;{ }^{1} \mathrm{H}$ NMR $\left(400 \mathrm{MHz}, \mathrm{CDCl}_{3}\right) \delta 7.76(\mathrm{~d}, J=7.9 \mathrm{~Hz}, 2 \mathrm{H}), 7.34-7.21(\mathrm{~m}$, 4H), $6.96(\mathrm{t}, J=7.3 \mathrm{~Hz}, 1 \mathrm{H}), 6.78(\mathrm{~d}, J=8.0 \mathrm{~Hz}, 2 \mathrm{H}), 5.02(\mathrm{brs}, 1 \mathrm{H}), 3.97(\mathrm{t}, J=5.1 \mathrm{~Hz}, 2 \mathrm{H}), 3.35(\mathrm{q}, J=$ $5.5 \mathrm{~Hz}, 2 \mathrm{H}), 2.41(\mathrm{~s}, 3 \mathrm{H}) ;{ }^{13} \mathrm{C} \mathrm{NMR}\left(101 \mathrm{MHz}, \mathrm{CDCl}_{3}\right) \delta 158.15,143.70,137.09,129.90,129.65,127.20$, 121.51, 114.58, 66.32, 42.73, 21.64; IR (neat): $v\left(\mathrm{~cm}^{-1}\right)$ 3294, 2920, 2850, 1598, 1328, 1151, 758, 670; HRMS (ESI+) m/z calcd. $\mathrm{C}_{15} \mathrm{H}_{18} \mathrm{NO}_{3} \mathrm{~S}^{+}[\mathrm{M}+\mathrm{H}]^{+}:$292.1002, found: 292.1001. 
$\mathrm{Ph}_{\mathrm{O}} \frown \mathrm{NHTs}$

\section{N-(2-(Benzyloxy)ethyl)-4-methylbenzenesulfonamide (3la)}

Yellow oil; ${ }^{1} \mathrm{H}$ NMR (400 MHz, $\left.\mathrm{CDCl}_{3}\right) \delta 7.72(\mathrm{~d}, J=7.9 \mathrm{~Hz}, 2 \mathrm{H}), 7.38-7.22(\mathrm{~m}, 7 \mathrm{H}), 4.89$ (brs, 1H), 4.42 (s, 2H), $3.49(\mathrm{t}, J=4.9 \mathrm{~Hz}, 2 \mathrm{H}), 3.14(\mathrm{q}, J=4.9 \mathrm{~Hz}, 2 \mathrm{H}), 2.42(\mathrm{~s}, 3 \mathrm{H}) ;{ }^{13} \mathrm{C} \mathrm{NMR}\left(101 \mathrm{MHz}, \mathrm{CDCl}_{3}\right) \delta$ 143.54, 137.60, 137.04, 129.83, 128.63, 128.07, 127.94, 127.21, 73.38, 68.42, 43.15, 21.64; IR (neat): $v\left(\mathrm{~cm}^{-}\right.$ $\left.{ }^{1}\right) 3284,2926,2855,1599,1320,1150,785,666$; HRMS (ESI+) m/z calcd. $\mathrm{C}_{16} \mathrm{H}_{20} \mathrm{NO}_{3} \mathrm{~S}^{+}[\mathrm{M}+\mathrm{H}]^{+}: 306.1158$, found: 306.1159 .

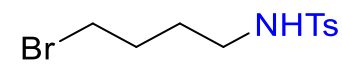

\section{$N$-(4-Bromobutyl)-4-methylbenzenesulfonamide (3ma)}

White solid, m.p.: $58.3-59.0{ }^{\circ} \mathrm{C} ;{ }^{1} \mathrm{H}$ NMR $\left(400 \mathrm{MHz}, \mathrm{CDCl}_{3}\right) \delta 7.75(\mathrm{~d}, J=8.1 \mathrm{~Hz}, 2 \mathrm{H}), 7.32(\mathrm{~d}, J=8.1 \mathrm{~Hz}$, 2H), $4.44(\mathrm{t}, J=7.5 \mathrm{~Hz}, 1 \mathrm{H}), 3.36(\mathrm{t}, J=6.5 \mathrm{~Hz}, 2 \mathrm{H}), 2.98(\mathrm{q}, J=6.7 \mathrm{~Hz}, 2 \mathrm{H}), 2.43(\mathrm{~s}, 3 \mathrm{H}), 1.93-1.81(\mathrm{~m}$, 2H), $1.69-1.57(\mathrm{~m}, 2 \mathrm{H}) ;{ }^{13} \mathrm{C}$ NMR $\left(101 \mathrm{MHz}, \mathrm{CDCl}_{3}\right) \delta 143.71,136.95,129.93,127.23,42.44,33.02$, 29.60, 28.29, 21.68; IR (neat): $v\left(\mathrm{~cm}^{-1}\right)$ 3303, 2974, 2922, 1597, 1331, 1156, 1091, 657; HRMS (ESI+) m/z calcd. $\mathrm{C}_{11} \mathrm{H}_{17} \mathrm{BrNO}_{2} \mathrm{~S}^{+}[\mathrm{M}+\mathrm{H}]^{+}:$306.0158, found: 306.0155 .

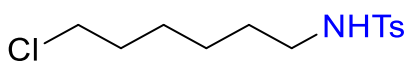

$N$-(6-Chlorohexyl)-4-methylbenzenesulfonamide (3na)

Yellow oil; ${ }^{1} \mathrm{H}$ NMR $\left(400 \mathrm{MHz}, \mathrm{CDCl}_{3}\right) \delta 7.75(\mathrm{~d}, J=7.8 \mathrm{~Hz}, 2 \mathrm{H}), 7.32(\mathrm{~d}, J=7.8 \mathrm{~Hz}, 2 \mathrm{H}), 4.61(\mathrm{~s}, J=6.2$ $\mathrm{Hz} 1 \mathrm{H}), 3.49(\mathrm{t}, J=6.7 \mathrm{~Hz}, 2 \mathrm{H}), 2.93(\mathrm{q}, J=6.8 \mathrm{~Hz}, 2 \mathrm{H}), 2.43(\mathrm{~s}, 3 \mathrm{H}), 1.74-1.67(\mathrm{~m}, 2 \mathrm{H}), 1.51-1.43(\mathrm{~m}$, 2H), $1.41-1.33(\mathrm{~m}, 2 \mathrm{H}), 1.32-1.24(\mathrm{~m}, 2 \mathrm{H}) ;{ }^{13} \mathrm{C} \mathrm{NMR}\left(101 \mathrm{MHz}, \mathrm{CDCl}_{3}\right) \delta 143.53,137.07,129.84$, 127.21, 44.99, 43.15, 32.43, 29.51, 26.41, 25.87, 21.64.; IR (neat): $v\left(\mathrm{~cm}^{-1}\right) 3280,2936,2861,1598,1321$, 1154, 814, 660; HRMS (ESI+) m/z calcd. $\mathrm{C}_{13} \mathrm{H}_{21} \mathrm{ClNO}_{2} \mathrm{~S}^{+}[\mathrm{M}+\mathrm{H}]^{+}:$290.0976, found: 290.0981.<smiles>[SH3-]NCCCCCCBr</smiles>

\section{$N$-(6-Bromohexyl)-4-methylbenzenesulfonamide (3oa)}

Colorless oil; ${ }^{1} \mathrm{H}$ NMR $\left(400 \mathrm{MHz}, \mathrm{CDCl}_{3}\right) \delta 7.75(\mathrm{~d}, J=7.8 \mathrm{~Hz}, 2 \mathrm{H}), 7.32(\mathrm{~d}, J=7.8 \mathrm{~Hz}, 2 \mathrm{H}), 4.60(\mathrm{t}, J=$ $6.3 \mathrm{~Hz}, 1 \mathrm{H}), 3.36(\mathrm{t}, J=6.7 \mathrm{~Hz}, 2 \mathrm{H}), 2.93(\mathrm{q}, J=6.8 \mathrm{~Hz}, 2 \mathrm{H}), 2.43(\mathrm{~s}, 3 \mathrm{H}), 1.82-1.75(\mathrm{~m}, 2 \mathrm{H}), 1.51-1.43$ $(\mathrm{m}, 2 \mathrm{H}), 1.41-1.33(\mathrm{~m}, 2 \mathrm{H}), 1.32-1.24(\mathrm{~m}, 2 \mathrm{H}) ;{ }^{13} \mathrm{C} \mathrm{NMR}\left(101 \mathrm{MHz}, \mathrm{CDCl}_{3}\right) \delta 143.54,137.07,129.84$, 
127.21, 43.14, 33.77, 32.59, 29.49, 27.68, 25.74, 21.65; IR (neat): $v\left(\mathrm{~cm}^{-1}\right) 3279,2934,2860,1598,1322$, 1155, 1092, 660; HRMS (ESI+) m/z calcd. $\mathrm{C}_{13} \mathrm{H}_{20} \mathrm{BrNNaO}_{2} \mathrm{~S}^{+}[\mathrm{M}+\mathrm{Na}]^{+}: 356.0290$, found: 356.0287 .

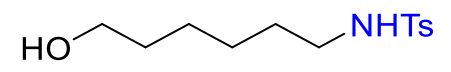

$N$-(6-Hydroxyhexyl)-4-methylbenzenesulfonamide (3pa)

Colorless oil; ${ }^{1} \mathrm{H}$ NMR (400 MHz, $\left.\mathrm{CDCl}_{3}\right) \delta 7.75(\mathrm{~d}, J=8.4 \mathrm{~Hz}, 2 \mathrm{H}), 7.31(\mathrm{~d}, J=8.3 \mathrm{~Hz}, 2 \mathrm{H}), 4.65(\mathrm{t}, J=$ $6.2 \mathrm{~Hz}, 1 \mathrm{H}), 3.61(\mathrm{t}, J=6.5 \mathrm{~Hz}, 2 \mathrm{H}), 2.93(\mathrm{q}, J=6.8 \mathrm{~Hz}, 2 \mathrm{H}), 2.43(\mathrm{~s}, 3 \mathrm{H}), 1.55-1.46(\mathrm{~m} 4 \mathrm{H}), 1.32-1.29$ (m, 4H); ${ }^{13} \mathrm{C}$ NMR $\left(101 \mathrm{MHz}, \mathrm{CDCl}_{3}\right) \delta 143.51,137.07,129.84,127.22,62.80,43.18,32.51,29.59,26.30$, 25.27, 21.65; IR (neat): $v\left(\mathrm{~cm}^{-1}\right)$ 3506, 3278, 2933, 2860, 1598, 1320, 1152, 1091, 660; HRMS (ESI+) m/z calcd. $\mathrm{C}_{13} \mathrm{H}_{21} \mathrm{NNaO}_{3} \mathrm{~S}^{+}[\mathrm{M}+\mathrm{Na}]^{+}:$294.1134, found: 294.1129 .<smiles>[NH3+]CCC(=O)OCc1ccccc1</smiles>

\section{Benzyl 3-((4-methylphenyl)sulfonamido)propanoate (3qa)}

Colorless oil; ${ }^{1} \mathrm{H}$ NMR (400 MHz, $\left.\mathrm{CDCl}_{3}\right) \delta 7.72(\mathrm{~d}, J=8.3 \mathrm{~Hz}, 2 \mathrm{H}), 7.41-7.25(\mathrm{~m}, 7 \mathrm{H}), 5.17(\mathrm{t}, J=6.6$ $\mathrm{Hz}, 1 \mathrm{H}), 5.09$ (s, 2H), $3.20(\mathrm{q}, J=6.4 \mathrm{~Hz}, 2 \mathrm{H}), 2.57(\mathrm{t}, J=6.0 \mathrm{~Hz}, 2 \mathrm{H}), 2.41(\mathrm{~s}, 3 \mathrm{H}) ;{ }^{13} \mathrm{C} \mathrm{NMR}(101 \mathrm{MHz}$, $\left.\mathrm{CDCl}_{3}\right) \delta 172.02,143.65,137.10,135.45,129.91,128.79,128.63,128.44,127.15,66.91,38.87,34.17,21.64$; IR (neat): $v\left(\mathrm{~cm}^{-1}\right) 3287,3034,2926,1730,1325,1155,660 ;$ HRMS (ESI+) m/z calcd. $\mathrm{C}_{17} \mathrm{H}_{20} \mathrm{NO}_{4} \mathrm{~S}^{+}[\mathrm{M}+\mathrm{H}]^{+}$: 334.1108, found: 334.1115 .<smiles></smiles>

\section{Methyl 2-methyl-3-((4-methylphenyl)sulfonamido)propanoate (3ra)}

Colorless oil; ${ }^{1} \mathrm{H}$ NMR (400 MHz, $\left.\mathrm{CDCl}_{3}\right) \delta 7.74(\mathrm{~d}, J=8.3 \mathrm{~Hz}, 2 \mathrm{H}), 7.31(\mathrm{~d}, J=8.3 \mathrm{~Hz}, 2 \mathrm{H}), 5.10(\mathrm{t}, J=$ $6.7 \mathrm{~Hz}, 1 \mathrm{H}), 3.66(\mathrm{~s}, 3 \mathrm{H}), 3.12-2.98(\mathrm{~m}, 2 \mathrm{H}), 2.74-2.61(\mathrm{~m}, 1 \mathrm{H}), 2.43(\mathrm{~s}, 3 \mathrm{H}), 1.16(\mathrm{~d}, J=7.3 \mathrm{~Hz}, 3 \mathrm{H})$; ${ }^{13} \mathrm{C}$ NMR $\left(101 \mathrm{MHz}, \mathrm{CDCl}_{3}\right) \delta 175.46,143.59,137.16,129.89,127.13,52.17,45.52,39.65,21.64,14.93 ; \mathrm{IR}$ (neat): $v\left(\mathrm{~cm}^{-1}\right) 3283,2922,2851,1727,1156,661 ; \mathrm{HRMS}(\mathrm{ESI}+) \mathrm{m} / \mathrm{z}$ calcd. $\mathrm{C}_{12} \mathrm{H}_{18} \mathrm{NO}_{4} \mathrm{~S}^{+}[\mathrm{M}+\mathrm{H}]^{+}$: 272.0951, found: 272.0959 .<smiles>CCCCCC(=O)CC[NH2+][SH3-]</smiles> 
White solid, m.p.: $111.8-112.9{ }^{\circ} \mathrm{C} ;{ }^{1} \mathrm{H}$ NMR $\left(400 \mathrm{MHz}, \mathrm{CDCl}_{3}\right) \delta 7.74(\mathrm{~d}, J=8.1 \mathrm{~Hz}, 2 \mathrm{H}), 7.31(\mathrm{~d}, J=8.1$ $\mathrm{Hz}, 2 \mathrm{H}), 5.10(\mathrm{t}, J=6.6 \mathrm{~Hz}, 1 \mathrm{H}), 3.18-3.09(\mathrm{~m}, 2 \mathrm{H}), 2.65(\mathrm{t}, J=5.7 \mathrm{~Hz}, 2 \mathrm{H}), 2.43(\mathrm{~s}, 3 \mathrm{H}), 2.34(\mathrm{t}, J=7.5$ $\mathrm{Hz}, 2 \mathrm{H}), 1.56-1.49(\mathrm{~m}, 2 \mathrm{H}), 1.37-1.16(\mathrm{~m}, 4 \mathrm{H}), 0.88(\mathrm{t}, J=7.1 \mathrm{~Hz}, 3 \mathrm{H}) ;{ }^{13} \mathrm{C} \mathrm{NMR}\left(101 \mathrm{MHz}, \mathrm{CDCl}_{3}\right) \delta$ $210.53,143.44,137.05,129.77,127.03,42.89,41.84,38.10,31.27,23.33,22.39,21.52,13.87$; IR (neat): $v$ $\left(\mathrm{cm}^{-1}\right)$ 3258, 2962, 2950, 2925, 2863, 1708, 1317, 1150, 816, 668; HRMS (ESI+) m/z calcd. $\mathrm{C}_{15} \mathrm{H}_{23} \mathrm{NNaO}_{3} \mathrm{~S}^{+}$ $[\mathrm{M}+\mathrm{Na}]^{+}:$320.1291, found: 320.1292 .<smiles>C=CCC[NH3+]</smiles>

$N$-(But-3-en-1-yl)-4-methylbenzenesulfonamide (3ta)

Pale yellow oil; ${ }^{1} \mathrm{H}$ NMR (400 MHz, $\left.\mathrm{CDCl}_{3}\right) \delta 7.75(\mathrm{~d}, J=7.9 \mathrm{~Hz}, 2 \mathrm{H}), 7.31(\mathrm{~d}, J=7.9 \mathrm{~Hz}, 2 \mathrm{H}), 5.70-5.55$ $(\mathrm{m}, 1 \mathrm{H}), 5.10-4.99(\mathrm{~m}, 2 \mathrm{H}), 4.59(\mathrm{t}, J=6.1 \mathrm{~Hz}, 1 \mathrm{H}), 3.01(\mathrm{q}, J=6.5 \mathrm{~Hz}, 2 \mathrm{H}), 2.43(\mathrm{~s}, 3 \mathrm{H}), 2.20(\mathrm{q}, J=6.8$ $\mathrm{Hz}, 2 \mathrm{H}) ;{ }^{13} \mathrm{C} \mathrm{NMR}\left(101 \mathrm{MHz}, \mathrm{CDCl}_{3}\right) \delta 143.55,137.06,134.30,129.83,127.23,118.22,42.21,33.72,21.63 ;$ IR (neat): $v\left(\mathrm{~cm}^{-1}\right)$ 3280, 2926, 1598, 1321, 1155, 1093, 660; HRMS (ESI+) m/z calcd. $\mathrm{C}_{11} \mathrm{H}_{15} \mathrm{NNaO}_{2} \mathrm{~S}^{+}$ $[\mathrm{M}+\mathrm{Na}]^{+}:$248.0716, found: 248.0719 .

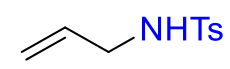

\section{$N$-Allyl-4-methylbenzenesulfonamide (3ua)}

Yellow oil; ${ }^{1} \mathrm{H}$ NMR $\left(400 \mathrm{MHz}, \mathrm{CDCl}_{3}\right) \delta 7.75(\mathrm{~d}, J=8.1 \mathrm{~Hz}, 2 \mathrm{H}), 7.32(\mathrm{~d}, J=8.1 \mathrm{~Hz}, 2 \mathrm{H}), 5.94-5.48(\mathrm{~m}$, 1H), $5.34-4.84(\mathrm{~m}, 2 \mathrm{H}), 4.39(\mathrm{brs}, 1 \mathrm{H}), 3.60-3.53(\mathrm{~m}, 2 \mathrm{H}), 2.44(\mathrm{~s}, 3 \mathrm{H}) ;{ }^{13} \mathrm{C} \mathrm{NMR}\left(101 \mathrm{MHz}, \mathrm{CDCl}_{3}\right) \delta$ 143.69, 137.09, 133.13, 129.89, 127.30, 117.89, 45.94, 21.67; IR (neat): $v\left(\mathrm{~cm}^{-1}\right)$ 3248, 2923, 2852, 1596, 1422, 1155, 664; HRMS (ESI+) m/z calcd. $\mathrm{C}_{10} \mathrm{H}_{14} \mathrm{NO}_{2} \mathrm{~S}^{+}[\mathrm{M}+\mathrm{H}]^{+}:$212.0740, found: 212.0748 .

\section{$\gtrsim \mathrm{NHTs}$}

$N$-(But-3-en-1-yl)-4-methylbenzenesulfonamide (3za)

${ }^{1} \mathrm{H}$ NMR $\left(400 \mathrm{MHz}, \mathrm{CDCl}_{3}\right) \delta 7.75(\mathrm{~d}, J=7.9 \mathrm{~Hz}, 2 \mathrm{H}), 7.31(\mathrm{~d}, J=7.9 \mathrm{~Hz}, 2 \mathrm{H}), 5.68-5.57$ (m, 1H), $5.10-$ $5.01(\mathrm{~m}, 2 \mathrm{H}), 4.59(\mathrm{t}, J=6.1 \mathrm{~Hz}, 1 \mathrm{H}), 3.02(\mathrm{q}, J=6.5 \mathrm{~Hz}, 2 \mathrm{H}), 2.43(\mathrm{~s}, 3 \mathrm{H}), 2.20(\mathrm{q}, J=6.8 \mathrm{~Hz}, 2 \mathrm{H}) ;{ }^{13} \mathrm{C}$ NMR $\left(101 \mathrm{MHz}, \mathrm{CDCl}_{3}\right) \delta 143.55,137.06,134.30,129.83,127.23,118.22,42.21,33.72,21.63$.<smiles>[AsH2]NCc1ccccc1</smiles> 


\section{$N$-Benzyl-4-methylbenzenesulfonamide (5aa)}

Colorless oil; ${ }^{1} \mathrm{H}$ NMR (400 MHz, $\left.\mathrm{CDCl}_{3}\right) \delta 7.76(\mathrm{~d}, J=7.9 \mathrm{~Hz}, 2 \mathrm{H}), 7.31$ (d, $\left.J=7.9 \mathrm{~Hz}, 2 \mathrm{H}\right), 7.29-7.24$ (m, 3H), $7.26-7.16$ (m, 2H), 4.70 (brs, 1H), 4.12 (d, $J=6.2 \mathrm{~Hz}, 2 \mathrm{H}), 2.44$ (s, 3H); ${ }^{13} \mathrm{C}$ NMR (101 MHz, $\left.\mathrm{CDCl}_{3}\right) \delta 143.69,136.98,136.39,129.89,128.84,128.07,128.01,127.33,47.42,21.68$; IR (neat): $v\left(\mathrm{~cm}^{-1}\right)$ $3279,2980,2935,2877,1598,1319,1155,814,659$; HRMS (ESI+) m/z calcd. $\mathrm{C}_{14} \mathrm{H}_{15} \mathrm{NNaO}_{2} \mathrm{~S}^{+}[\mathrm{M}+\mathrm{Na}]^{+}$: 284.0716, found: 284.0722 .<smiles>Fc1ccc(CNS)cc1</smiles>

$N$-(4-Fluorobenzyl)-4-methylbenzenesulfonamide (5ba)

White solid, m.p.: $99.4-100.3{ }^{\circ} \mathrm{C} ;{ }^{1} \mathrm{H}$ NMR $\left(400 \mathrm{MHz}, \mathrm{CDCl}_{3}\right) \delta 7.74(\mathrm{~d}, J=8.0 \mathrm{~Hz}, 2 \mathrm{H}), 7.31(\mathrm{~d}, J=8.0$ Hz, 2H), $7.17(\mathrm{dd}, J=8.4,5.4 \mathrm{~Hz}, 2 \mathrm{H}), 6.96(\mathrm{t}, J=8.5 \mathrm{~Hz}, 2 \mathrm{H}), 4.78(\mathrm{t}, J=6.4 \mathrm{~Hz}, 1 \mathrm{H}), 4.09(\mathrm{~d}, J=6.2 \mathrm{~Hz}$, 2H), $2.44(\mathrm{~s}, 3 \mathrm{H}) ;{ }^{13} \mathrm{C}$ NMR $\left(101 \mathrm{MHz}, \mathrm{CDCl}_{3}\right) \delta 162.52(\mathrm{~d}, J=246.7 \mathrm{~Hz}), 143.79,136.97,132.24(\mathrm{~d}, J=$ 3.6 Hz), 129.91, $129.76(\mathrm{~d}, J=8.1 \mathrm{~Hz}), 127.29,115.70(\mathrm{~d}, J=21.7 \mathrm{~Hz}), 46.70,21.68$; IR (neat): $v\left(\mathrm{~cm}^{-1}\right)$ 3244, 2961, 2921, 1606, 1507, 1316, 1150, 1065, 811; HRMS (ESI+) m/z calcd. $\mathrm{C}_{14} \mathrm{H}_{14} \mathrm{FNNaO}_{2} \mathrm{~S}^{+}[\mathrm{M}+\mathrm{Na}]^{+}$: 302.0621, found: 302.0624 .<smiles>[15NH2]NCc1ccc(Cl)cc1</smiles>

$N$-(4-Chlorobenzyl)-4-methylbenzenesulfonamide (5ca)

White solid, m.p.: $98.4-99.2^{\circ} \mathrm{C} ;{ }^{1} \mathrm{H}$ NMR $\left(400 \mathrm{MHz}, \mathrm{CDCl}_{3}\right) \delta 7.73(\mathrm{~d}, J=8.1 \mathrm{~Hz}, 2 \mathrm{H}), 7.30(\mathrm{~d}, J=8.1 \mathrm{~Hz}$, 2H), $7.24(\mathrm{~d}, J=8.5 \mathrm{~Hz}, 2 \mathrm{H}), 7.13(\mathrm{~d}, J=8.5 \mathrm{~Hz}, 2 \mathrm{H}), 4.82(\mathrm{t}, J=6.4 \mathrm{~Hz}, 1 \mathrm{H}), 4.09(\mathrm{~d}, J=6.3 \mathrm{~Hz}, 2 \mathrm{H})$, $2.44(\mathrm{~s}, 3 \mathrm{H}) ;{ }^{13} \mathrm{C}$ NMR $\left(101 \mathrm{MHz}, \mathrm{CDCl}_{3}\right) \delta 143.84,136.92,134.99,133.90,129.92,129.35,128.95,127.28$, 46.71, 21.68; IR (neat): $v$ (cm ${ }^{-1}$ ) 3230, 2955, 2919, 2851, 1446, 1318, 1154, 659; HRMS (ESI+) m/z calcd. $\mathrm{C}_{14} \mathrm{H}_{14} \mathrm{ClNNaO}_{2} \mathrm{~S}^{+}[\mathrm{M}+\mathrm{Na}]^{+}: 318.0326$, found: 318.0325 .<smiles>SNCc1ccc(Br)cc1</smiles>

\section{$N$-(4-Bromobenzyl)-4-methylbenzenesulfonamide (5da)}

White solid, m.p.: $98.2-99.5^{\circ} \mathrm{C} ;{ }^{1} \mathrm{H}$ NMR $\left(400 \mathrm{MHz}, \mathrm{CDCl}_{3}\right) \delta 7.72(\mathrm{~d}, J=8.1 \mathrm{~Hz}, 2 \mathrm{H}), 7.38(\mathrm{~d}, J=8.4 \mathrm{~Hz}$, 2H), $7.29(\mathrm{~d}, J=8.1 \mathrm{~Hz}, 2 \mathrm{H}), 7.07(\mathrm{~d}, J=8.4 \mathrm{~Hz}, 2 \mathrm{H}), 4.88(\mathrm{t}, J=6.3 \mathrm{~Hz}, 1 \mathrm{H}), 4.07(\mathrm{~d}, J=6.4 \mathrm{~Hz}, 2 \mathrm{H})$, 
$2.44(\mathrm{~s}, 3 \mathrm{H}) ;{ }^{13} \mathrm{C} \mathrm{NMR}\left(101 \mathrm{MHz}, \mathrm{CDCl}_{3}\right) \delta 143.84,136.91,135.52,131.89,129.92,129.67,127.27,121.97$, 46.74, 21.69; IR (neat): $v\left(\mathrm{~cm}^{-1}\right) 3271,2957,2921,1450,1154,1064,811,660$; HRMS (ESI+) m/z calcd. $\mathrm{C}_{14} \mathrm{H}_{15} \mathrm{BrNO}_{2} \mathrm{~S}^{+}[\mathrm{M}+\mathrm{H}]^{+}: 340.0001$, found: 339.9995 .<smiles>Fc1ccc(CN[15F])c(F)c1</smiles>

$N$-(2,4-Difluorobenzyl)-4-methylbenzenesulfonamide (5ea)

Pale yellow oil; ${ }^{1} \mathrm{H}$ NMR (400 MHz, $\left.\mathrm{CDCl}_{3}\right) \delta 7.70(\mathrm{~d}, J=8.1 \mathrm{~Hz}, 2 \mathrm{H}), 7.27(\mathrm{~d}, J=8.1 \mathrm{~Hz}, 2 \mathrm{H}), 7.25-7.20$ $(\mathrm{m}, 1 \mathrm{H}), 6.87-6.52(\mathrm{~m}, 2 \mathrm{H}), 4.84(\mathrm{t}, J=6.5 \mathrm{~Hz}, 1 \mathrm{H}), 4.17(\mathrm{~d}, J=6.5 \mathrm{~Hz}, 2 \mathrm{H}), 2.42(\mathrm{~s}, 3 \mathrm{H}) ;{ }^{13} \mathrm{C} \mathrm{NMR}(101$ $\left.\mathrm{MHz}, \mathrm{CDCl}_{3}\right) \delta 162.78(\mathrm{dd}, J=249.3,11.7 \mathrm{~Hz}), 160.84(\mathrm{dd}, J=249.3,11.7 \mathrm{~Hz}), 143.75,137.00,131.21(\mathrm{dd}$, $J=9.5,5.7 \mathrm{~Hz}), 129.82,127.20,119.78(\mathrm{dd}, J=14.6,3.7 \mathrm{~Hz}), 111.53(\mathrm{dd}, J=21.1,3.6 \mathrm{~Hz}), 103.96(\mathrm{t}, J=$ $25.4 \mathrm{~Hz}), 40.89$ (d, $J=3.5 \mathrm{~Hz}), 21.63$; IR (neat): $v\left(\mathrm{~cm}^{-1}\right)$ 3263, 2978, 2845, 1319, 1149, 854, 660; HRMS (ESI+) $\mathrm{m} / \mathrm{z}$ calcd. $\mathrm{C}_{14} \mathrm{H}_{13} \mathrm{~F}_{2} \mathrm{NNaO}_{2} \mathrm{~S}^{+}[\mathrm{M}+\mathrm{Na}]^{+}$: 320.0527 , found: 320.0535 .<smiles>FC(F)(F)c1ccccc1CN[13F]</smiles>

4-Methyl- $N$-(2-(trifluoromethyl)benzyl)benzenesulfonamide (5fa)

Yellow oil; ${ }^{1} \mathrm{H}$ NMR $\left(400 \mathrm{MHz}, \mathrm{CDCl}_{3}\right) \delta 7.75(\mathrm{~d}, J=8.4 \mathrm{~Hz}, 2 \mathrm{H}), 7.59(\mathrm{t}, J=7.8 \mathrm{~Hz}, 2 \mathrm{H}), 7.50(\mathrm{t}, J=8.1$ $\mathrm{Hz}, 1 \mathrm{H}), 7.38(\mathrm{t}, J=7.6 \mathrm{~Hz}, 1 \mathrm{H}) .7 .31(\mathrm{~d}, J=7.9 \mathrm{~Hz}, 2 \mathrm{H}), 4.79(\mathrm{t}, J=6.6 \mathrm{~Hz}, 1 \mathrm{H}), 4.30(\mathrm{~d}, J=6.6 \mathrm{~Hz}, 2 \mathrm{H})$, $2.44(\mathrm{~s}, 3 \mathrm{H}) ;{ }^{13} \mathrm{C}$ NMR $\left(101 \mathrm{MHz}, \mathrm{CDCl}_{3}\right) \delta 143.85,136.97,135.04,132.50,130.96,129.94,128.19(\mathrm{q}, J=$ $30.5 \mathrm{~Hz}), 128.16,127.24,127.23,126.13$ (q, $J=5.4 \mathrm{~Hz}), 124.33$ (q, $J=273.9 \mathrm{~Hz}), 43.89,21.69$; IR (neat): $v$ $\left(\mathrm{cm}^{-1}\right)$ 3283, 2928, 2854, 1321, 1150, 844, 666; HRMS (ESI+) m/z calcd. $\mathrm{C}_{15} \mathrm{H}_{14} \mathrm{~F}_{3} \mathrm{NNaO}_{2} \mathrm{~S}^{+}[\mathrm{M}+\mathrm{Na}]^{+}$: 352.0590, found: 352.0584 .<smiles></smiles>

\section{4-Methyl- $N$-(2-methylbenzyl)benzenesulfonamide (5ga)}

White solid, m.p.: 112.6-113.8 ${ }^{\circ} \mathrm{C} ;{ }^{1} \mathrm{H}$ NMR $\left(400 \mathrm{MHz}, \mathrm{CDCl}_{3}\right) \delta 7.77(\mathrm{~d}, J=7.9 \mathrm{~Hz}, 2 \mathrm{H}), 7.32(\mathrm{~d}, J=7.9$ Hz, 2H), $7.23-7.08(\mathrm{~m}, 4 \mathrm{H}), 4.44(\mathrm{t}, J=6.0 \mathrm{~Hz}, 1 \mathrm{H}), 4.09$ (d, $J=5.9 \mathrm{~Hz}, 2 \mathrm{H}), 2.45$ (s, 3H), 2.25 (s, 3H); ${ }^{13} \mathrm{C}$ NMR $\left(101 \mathrm{MHz}, \mathrm{CDCl}_{3}\right) \delta 143.71,136.89,134.00,130.78,129.89,129.01,128.43,127.36,126.36$, 
45.58, 21.70, 18.93; IR (neat): $v\left(\mathrm{~cm}^{-1}\right)$ 3264, 2971, 2922, 1425, 1318, 1160, 703; HRMS (ESI+) m/z calcd. $\mathrm{C}_{15} \mathrm{H}_{17} \mathrm{NNaO}_{2} \mathrm{~S}^{+}[\mathrm{M}+\mathrm{Na}]^{+}:$298.0872, found: 298.0879.<smiles>Cc1ccc(CN[AsH3])cc1</smiles>

4-Methyl- $N$-(4-methylbenzyl)benzenesulfonamide (5ha)

White solid, m.p.: $88.5-89.1{ }^{\circ} \mathrm{C} ;{ }^{1} \mathrm{H}$ NMR $\left(400 \mathrm{MHz}, \mathrm{CDCl}_{3}\right) \delta 7.76(\mathrm{~d}, J=7.9 \mathrm{~Hz}, 2 \mathrm{H}), 7.31(\mathrm{~d}, J=7.9 \mathrm{~Hz}$, 2H), $7.10-7.06(\mathrm{~m}, 4 \mathrm{H}), 4.57(\mathrm{t}, J=6.1 \mathrm{~Hz}, 1 \mathrm{H}), 4.07(\mathrm{~d}, J=6.0 \mathrm{~Hz}, 2 \mathrm{H}), 2.44(\mathrm{~s}, 3 \mathrm{H}), 2.31(\mathrm{~s}, 3 \mathrm{H}) ;{ }^{13} \mathrm{C}$ NMR (101 MHz, $\left.\mathrm{CDCl}_{3}\right) \delta 143.62,137.85,137.00,133.33,129.86,129.50,128.00,127.34,47.19,21.67$, 21.22; IR (neat): $v\left(\mathrm{~cm}^{-1}\right) 3273,2918,2852,1325,1153,844,660 ; \mathrm{HRMS}$ (ESI+) m/z calcd. $\mathrm{C}_{15} \mathrm{H}_{17} \mathrm{NNaO}_{2} \mathrm{~S}^{+}$ $[\mathrm{M}+\mathrm{Na}]^{+}:$298.0872, found: 298.0868 .<smiles>[NH3+]Cc1ccc(-c2ccccc2)cc1</smiles>

$N$-([1,1'-Biphenyl]-4-ylmethyl)-4-methylbenzenesulfonamide (5ia)

White solid, m.p.: $107.8-108.3{ }^{\circ} \mathrm{C} ;{ }^{1} \mathrm{H}$ NMR $\left(400 \mathrm{MHz}, \mathrm{CDCl}_{3}\right) \delta 7.77(\mathrm{~d}, J=7.9 \mathrm{~Hz}, 2 \mathrm{H}), 7.59(\mathrm{~d}, J=7.8$ $\mathrm{Hz}, 1 \mathrm{H}), 7.54(\mathrm{~d}, J=7.6 \mathrm{~Hz}, 2 \mathrm{H}), 7.50(\mathrm{~d}, J=7.9 \mathrm{~Hz}, 2 \mathrm{H}), 7.43(\mathrm{t}, J=7.7 \mathrm{~Hz}, 2 \mathrm{H}), 7.36(\mathrm{~d}, J=7.3 \mathrm{~Hz}, 1 \mathrm{H})$, $7.31(\mathrm{~d}, J=8.1 \mathrm{~Hz}, 2 \mathrm{H}), 7.26(\mathrm{~d}, J=7.3 \mathrm{~Hz}, 1 \mathrm{H}), 4.75(\mathrm{brs}, 1 \mathrm{H}), 4.17(\mathrm{~d}, J=5.9 \mathrm{~Hz}, 2 \mathrm{H}), 2.43(\mathrm{~s}, 3 \mathrm{H}) ;{ }^{13} \mathrm{C}$ NMR $\left(101 \mathrm{MHz}, \mathrm{CDCl}_{3}\right) \delta 143.71,141.07,140.64,135.39,129.90,128.95,128.47,127.61,127.55,127.47$, 127.35, 127.23, 127.18, 47.15, 21.68; IR (neat): $v\left(\mathrm{~cm}^{-1}\right)$ 3283, 2969, 2923, 1323, 1152, 1071, 760, 665; HRMS (ESI+) m/z calcd. $\mathrm{C}_{20} \mathrm{H}_{20} \mathrm{NO}_{2} \mathrm{~S}^{+}[\mathrm{M}+\mathrm{H}]^{+}: 338.1209$, found: 338.1210 .<smiles>CC(C)(C)c1ccc(CN[AsH3])cc1</smiles>

$N$-(4-(Tert-butyl)benzyl)-4-methylbenzenesulfonamide (5ja)

White solid, m.p.: $111.6-112.9{ }^{\circ} \mathrm{C} ;{ }^{1} \mathrm{H}$ NMR $\left(400 \mathrm{MHz}, \mathrm{CDCl}_{3}\right) \delta 7.75(\mathrm{~d}, J=7.9 \mathrm{~Hz}, 2 \mathrm{H}), 7.29(\mathrm{~d}, J=7.9$ Hz, 4H), $7.11(\mathrm{~d}, J=7.9 \mathrm{~Hz}, 2 \mathrm{H}), 4.64(\mathrm{t}, J=6.6 \mathrm{~Hz}, 1 \mathrm{H}), 4.09$ (d, $J=6.0 \mathrm{~Hz}, 2 \mathrm{H}), 2.43(\mathrm{~s}, 3 \mathrm{H}), 1.28$ (s, 9H); ${ }^{13} \mathrm{C}$ NMR $\left(101 \mathrm{MHz}, \mathrm{CDCl}_{3}\right) \delta 151.16,143.56,137.06,133.33,129.84,127.80,127.34,125.74,47.11$, 34.66, 31.42, 21.67; IR (neat): $v\left(\mathrm{~cm}^{-1}\right)$ 3271, 2962, 2921, 1429, 1321, 1149, 1057, 688; HRMS (ESI+) m/z calcd. $\mathrm{C}_{18} \mathrm{H}_{23} \mathrm{NNaO}_{2} \mathrm{~S}^{+}[\mathrm{M}+\mathrm{Na}]^{+}: 340.1342$, found: 340.1344 . 
OMe $N H T s$

$N$-(3-Methoxybenzyl)-4-methylbenzenesulfonamide (5ka)

Pale yellow oil; ${ }^{1} \mathrm{H}$ NMR $\left(400 \mathrm{MHz}, \mathrm{CDCl}_{3}\right) \delta 7.76(\mathrm{~d}, J=7.9 \mathrm{~Hz}, 2 \mathrm{H}), 7.31(\mathrm{~d}, J=7.9 \mathrm{~Hz}, 2 \mathrm{H}), 7.19(\mathrm{t}, J=$ $7.9 \mathrm{~Hz}, 1 \mathrm{H}), 6.83-6.69(\mathrm{~m}, 3 \mathrm{H}), 4.64(\mathrm{t}, J=6.2 \mathrm{~Hz}, 1 \mathrm{H}), 4.10(\mathrm{~d}, J=6.2 \mathrm{~Hz}, 2 \mathrm{H}), 3.75(\mathrm{~s}, 3 \mathrm{H}), 2.44$ (s, 3H); ${ }^{13} \mathrm{C}$ NMR $\left(101 \mathrm{MHz}, \mathrm{CDCl}_{3}\right) \delta 160.01,143.71,137.93,137.02,129.90,127.35,120.17,113.82,113.30$, 55.35, 47.41, 21.67; IR (neat): $v\left(\mathrm{~cm}^{-1}\right)$ 3255, 2958, 2920, 2842, 1585, 1158, 1067, 666; HRMS (ESI+) m/z calcd. $\mathrm{C}_{15} \mathrm{H}_{17} \mathrm{NNaO}_{3} \mathrm{~S}^{+}[\mathrm{M}+\mathrm{Na}]^{+}: 314.0821$, found: 314.0825 .<smiles>CCOC(=O)CC(C)[18F]</smiles>

Ethyl 3-((4-methylphenyl)sulfonamido)butanoate (5la)

Colorless oil; ${ }^{1} \mathrm{H}$ NMR $\left(400 \mathrm{MHz}, \mathrm{CDCl}_{3}\right) \delta 7.76(\mathrm{~d}, J=7.8 \mathrm{~Hz}, 2 \mathrm{H}), 7.30(\mathrm{~d}, J=7.9 \mathrm{~Hz}, 2 \mathrm{H}), 5.24(\mathrm{~d}, J=$ $8.6 \mathrm{~Hz}, 1 \mathrm{H}), 4.14-4.01(\mathrm{~m}, 2 \mathrm{H}), 3.73-3.63(\mathrm{~m}, 1 \mathrm{H}), 2.43(\mathrm{~s}, 3 \mathrm{H}), 2.42(\mathrm{~d}, J=6.7 \mathrm{~Hz}, 2 \mathrm{H}), 1.22(\mathrm{t}, J=7.2$ $\mathrm{Hz}, 3 \mathrm{H}), 1.14(\mathrm{~d}, J=6.7 \mathrm{~Hz}, 3 \mathrm{H}) ;{ }^{13} \mathrm{C} \mathrm{NMR}\left(101 \mathrm{MHz}, \mathrm{CDCl}_{3}\right) \delta 171.33,143.48,138.13,129.83,127.17$, 60.88, 46.73, 40.81, 21.65, 21.17, 14.24; IR (neat): $v\left(\mathrm{~cm}^{-1}\right) 3277,2981,2934,1730,1303,1157,1087,662$; HRMS (ESI+) m/z calcd. $\mathrm{C}_{13} \mathrm{H}_{19} \mathrm{NNaO}_{4} \mathrm{~S}^{+}[\mathrm{M}+\mathrm{Na}]^{+}$: 308.0927 , found: 308.0925 .<smiles>COC(=O)CC(C)[NH3+]</smiles>

\section{Methyl 3-((4-methylphenyl)sulfonamido)butanoate (5ma)}

Colorless oil; ${ }^{1} \mathrm{H}$ NMR $\left(400 \mathrm{MHz}, \mathrm{CDCl}_{3}\right) \delta 7.76(\mathrm{~d}, J=8.4 \mathrm{~Hz}, 2 \mathrm{H}), 7.30(\mathrm{~d}, J=8.4 \mathrm{~Hz}, 2 \mathrm{H}), 5.15(\mathrm{~d}, J=$ $8.6 \mathrm{~Hz}, 1 \mathrm{H}), 3.74-3.65(\mathrm{~m}, 1 \mathrm{H}), 3.62(\mathrm{~s}, 3 \mathrm{H}), 2.44(\mathrm{~d}, J=6.7 \mathrm{~Hz} 2 \mathrm{H}), 2.43(\mathrm{~s}, 3 \mathrm{H}), 1.14(\mathrm{~d}, J=6.7 \mathrm{~Hz}, 3 \mathrm{H})$; ${ }^{13} \mathrm{C}$ NMR $\left(101 \mathrm{MHz}, \mathrm{CDCl}_{3}\right) \delta 171.73,143.52,138.10,129.85,127.18,51.88,46.69,40.64,21.66,21.21$; IR (neat): $v\left(\mathrm{~cm}^{-1}\right) 3278,2954,1732,1157,662$; HRMS (ESI+) m/z calcd. $\mathrm{C}_{12} \mathrm{H}_{18} \mathrm{NO}_{4} \mathrm{~S}^{+}[\mathrm{M}+\mathrm{H}]^{+}:$: 272.0951, found: 272.0955 .<smiles>[AsH3-]NC1Cc2ccccc2C1</smiles>

$N$-(2,3-Dihydro-1H-inden-2-yl)-4-methylbenzenesulfonamide (5na) 
Yellow oil; ${ }^{1} \mathrm{H}$ NMR $\left(400 \mathrm{MHz}, \mathrm{CDCl}_{3}\right) \delta 7.78(\mathrm{~d}, J=8.1 \mathrm{~Hz}, 2 \mathrm{H}), 7.33(\mathrm{~d}, J=8.1 \mathrm{~Hz}, 2 \mathrm{H}), 7.14(\mathrm{~s}, 4 \mathrm{H})$, $4.72(\mathrm{~d}, J=8.3 \mathrm{~Hz}, 1 \mathrm{H}), 4.18-4.06(\mathrm{~m}, 1 \mathrm{H}), 3.11(\mathrm{dd}, J=15.9,7.1 \mathrm{~Hz}, 2 \mathrm{H}), 2.74(\mathrm{dd}, J=15.8,5.8 \mathrm{~Hz}, 2 \mathrm{H})$, $2.45(\mathrm{~s}, 3 \mathrm{H}) ;{ }^{13} \mathrm{C}$ NMR $\left(101 \mathrm{MHz}, \mathrm{CDCl}_{3}\right) \delta 143.67,140.15,137.89,129.94,127.28,127.09,124.78,54.85$, 40.50, 21.71; IR (neat): $v\left(\mathrm{~cm}^{-1}\right)$ 3281, 2954, 2877, 1321, 1150, 666; HRMS (ESI+) m/z calcd. $\mathrm{C}_{16} \mathrm{H}_{17} \mathrm{NNaO}_{2} \mathrm{~S}^{+}[\mathrm{M}+\mathrm{Na}]^{+}: 310.0872$, found: 310.0873 .

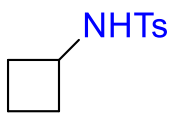

\section{$N$-Cyclobutyl-4-methylbenzenesulfonamide (5oa)}

Yellow oil; ${ }^{1} \mathrm{H}$ NMR $\left(400 \mathrm{MHz}, \mathrm{CDCl}_{3}\right) \delta 7.74(\mathrm{~d}, J=8.3 \mathrm{~Hz}, 2 \mathrm{H}), 7.29(\mathrm{~d}, J=8.0 \mathrm{~Hz}, 2 \mathrm{H}), 4.77(\mathrm{~d}, J=9.0$ $\mathrm{Hz}, 1 \mathrm{H}), 3.86-3.71(\mathrm{~m}, 1 \mathrm{H}), 2.42(\mathrm{~s}, 3 \mathrm{H}), 2.18-2.05(\mathrm{~m}, 2 \mathrm{H}), 1.84-1.68(\mathrm{~m}, 2 \mathrm{H}), 1.68-1.46(\mathrm{~m}, 2 \mathrm{H})$; ${ }^{13} \mathrm{C} \mathrm{NMR}\left(101 \mathrm{MHz}, \mathrm{CDCl}_{3}\right) \delta 143.49,138.02,129.78,127.16,48.27,31.85,21.65,15.10$; IR (neat): $v\left(\mathrm{~cm}^{-1}\right)$ 3268, 2924, 1304, 1159, 1142, 666; HRMS (ESI+) m/z calcd. $\mathrm{C}_{11} \mathrm{H}_{16} \mathrm{NO}_{2} \mathrm{~S}^{+}[\mathrm{M}+\mathrm{H}]^{+}:$: 226.0896, found: 226.0899.

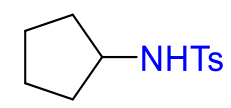

\section{$N$-Cyclopentyl-4-methylbenzenesulfonamide (5pa)}

Yellow oil; ${ }^{1} \mathrm{H}$ NMR (400 MHz, DMSO) $\delta 7.76(\mathrm{~d}, J=7.9 \mathrm{~Hz}, 2 \mathrm{H}), 7.30(\mathrm{~d}, J=7.9 \mathrm{~Hz}, 2 \mathrm{H}), 4.33(\mathrm{~d}, J=7.2$ $\mathrm{Hz}, 1 \mathrm{H}), 3.59(\mathrm{q}, J=6.8 \mathrm{~Hz}, 1 \mathrm{H}), 2.43(\mathrm{~s}, 3 \mathrm{H}), 1.84-1.74(\mathrm{~m}, 2 \mathrm{H}), 1.65-1.60(\mathrm{~m}, 2 \mathrm{H}), 1.53-1.42(\mathrm{~m}$, 2H), $1.38-1.31(\mathrm{~m}, 2 \mathrm{H}) ;{ }^{13} \mathrm{C}$ NMR $\left(101 \mathrm{MHz}, \mathrm{CDCl}_{3}\right) \delta 143.39,138.00,129.79,127.27,55.29,33.63$, 23.28, 21.67; IR (neat): $v\left(\mathrm{~cm}^{-1}\right) 3271,2959,2871,1320,1153,663$; HRMS (ESI+) m/z calcd. $\mathrm{C}_{12} \mathrm{H}_{18} \mathrm{NO}_{2} \mathrm{~S}^{+}$ $[\mathrm{M}+\mathrm{H}]^{+}:$240.1053, found: 240.1055 .

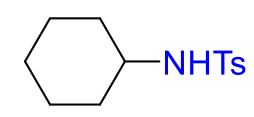

\section{$N$-Cyclohexyl-4-methylbenzenesulfonamide (5qa)}

Yellow oil; ${ }^{1} \mathrm{H}$ NMR $\left(400 \mathrm{MHz}, \mathrm{CDCl}_{3}\right) \delta 7.75(\mathrm{~d}, J=8.3 \mathrm{~Hz}, 2 \mathrm{H}), 7.29(\mathrm{~d}, J=8.3 \mathrm{~Hz}, 2 \mathrm{H}), 4.32(\mathrm{~d}, J=7.7$ $\mathrm{Hz}, 1 \mathrm{H}), 3.17-3.08(\mathrm{~m}, 1 \mathrm{H}), 2.42(\mathrm{~s}, 3 \mathrm{H}), 1.78-1.71(\mathrm{~m}, 2 \mathrm{H}), 1.65-1.59(\mathrm{~m}, 2 \mathrm{H}), 1.54-1.47(\mathrm{~m}, 1 \mathrm{H})$, $1.30-1.03(\mathrm{~m}, 5 \mathrm{H}) ;{ }^{13} \mathrm{C}$ NMR $\left(101 \mathrm{MHz}, \mathrm{CDCl}_{3}\right) \delta 143.28,138.62,129.78,127.08,52.72,34.13,25.30$, 24.78, 21.66; IR (neat): $v\left(\mathrm{~cm}^{-1}\right) 3275,2930,2855,1451,1157,663$; HRMS (ESI+) m/z calcd. $\mathrm{C}_{13} \mathrm{H}_{20} \mathrm{NO}_{2} \mathrm{~S}^{+}$ $[\mathrm{M}+\mathrm{H}]^{+}:$254.1209, found: 254.1206. 
<smiles>O=[N+](NCCCCc1ccccc1)c1ccccc1</smiles>

\section{$N$-(4-Phenylbutyl)benzenesulfonamide (3ab)}

Yellow oil; ${ }^{1} \mathrm{H}$ NMR $\left(400 \mathrm{MHz}, \mathrm{CDCl}_{3}\right) \delta 7.88-7.81(\mathrm{~m}, 2 \mathrm{H}), 7.62-7.54(\mathrm{~m}, 1 \mathrm{H}), 7.53-7.47(\mathrm{~m}, 2 \mathrm{H})$, $7.30-7.21(\mathrm{~m}, 2 \mathrm{H}), 7.19-7.14(\mathrm{~m}, 1 \mathrm{H}), 7.12-7.06(\mathrm{~m}, 2 \mathrm{H}), 4.46(\mathrm{t}, J=6.1 \mathrm{~Hz}, 1 \mathrm{H}), 2.97(\mathrm{q}, J=6.7 \mathrm{~Hz}$ $2 \mathrm{H}), 2.55(\mathrm{t}, J=7.5 \mathrm{~Hz}, 2 \mathrm{H}), 1.66-1.54(\mathrm{~m}, 2 \mathrm{H}), 1.52-1.43(\mathrm{~m}, 2 \mathrm{H}) ;{ }^{13} \mathrm{C} \mathrm{NMR}\left(101 \mathrm{MHz}, \mathrm{CDCl}_{3}\right) \delta$ $141.86,140.09,132.75,129.25,128.50,128.48,127.15,126.02,43.23,35.35,29.25,28.33$; IR (neat): $v$ (cm 1) 3280, 2935, 2860, 1447, 1154, 669; HRMS (ESI+) m/z calcd. $\mathrm{C}_{16} \mathrm{H}_{20} \mathrm{NO}_{2} \mathrm{~S}^{+}[\mathrm{M}+\mathrm{H}]^{+}$: 290.1209, found: 290.1214.<smiles>O=[N+](NCCCCc1ccccc1)c1ccccc1F</smiles>

\section{2-Fluoro- $N$-(4-phenylbutyl)benzenesulfonamide (3ac)}

Pale yellow oil; ${ }^{1} \mathrm{H}$ NMR $\left(400 \mathrm{MHz}, \mathrm{CDCl}_{3}\right) \delta 7.89(\mathrm{td}, J=7.6,1.9 \mathrm{~Hz}, 1 \mathrm{H}), 7.62-7.52(\mathrm{~m}, 1 \mathrm{H}), 7.34-$ $7.22(\mathrm{~m}, 3 \mathrm{H}), 7.24-7.13(\mathrm{~m}, 2 \mathrm{H}), 7.13-7.08(\mathrm{~m}, 2 \mathrm{H}), 4.68(\mathrm{brs}, 1 \mathrm{H}), 3.01(\mathrm{q}, J=6.7 \mathrm{~Hz}, 2 \mathrm{H}), 2.56(\mathrm{t}, J=$ $7.4 \mathrm{~Hz}, 2 \mathrm{H}), 1.70-1.55(\mathrm{~m}, 2 \mathrm{H}), 1.54-1.47(\mathrm{~m}, 2 \mathrm{H}) ;{ }^{13} \mathrm{C} \mathrm{NMR}\left(101 \mathrm{MHz}, \mathrm{CDCl}_{3}\right) \delta 158.89(\mathrm{~d}, J=255.0$ $\mathrm{Hz}), 141.81,135.03(\mathrm{~d}, J=8.7 \mathrm{~Hz}), 130.55,128.49(\mathrm{~d}, J=2.9 \mathrm{~Hz}), 126.04,124.66(\mathrm{~d}, J=4.3 \mathrm{~Hz}), 117.03(\mathrm{~d}$, $J=21.1 \mathrm{~Hz}$ ), 43.31, 35.35, 29.25, 28.28; IR (neat): $v\left(\mathrm{~cm}^{-1}\right)$ 3297, 3026, 2934, 2860, 1599, 1474, 1331, 1165 , 762, 696; HRMS (ESI+) m/z calcd. $\mathrm{C}_{16} \mathrm{H}_{19} \mathrm{FNO}_{2} \mathrm{~S}^{+}[\mathrm{M}+\mathrm{H}]^{+}: 308.1115$, found: 308.1109 .<smiles>O=[N+](NCCCCc1ccccc1)c1ccccc1Cl</smiles>

\section{2-Chloro- $N$-(4-phenylbutyl)benzenesulfonamide (3ad)}

Pale yellow oil; ${ }^{1} \mathrm{H}$ NMR (400 MHz, $\left.\mathrm{CDCl}_{3}\right) \delta 8.09(\mathrm{~d}, J=8.6 \mathrm{~Hz}, 1 \mathrm{H}), 7.56-7.48(\mathrm{~m}, 2 \mathrm{H}), 7.46-7.37(\mathrm{~m}$, 1H), $7.30-7.22(\mathrm{~m}, 2 \mathrm{H}), 7.22-7.14(\mathrm{~m}, 1 \mathrm{H}), 7.14-7.06(\mathrm{~m}, 2 \mathrm{H}), 4.94$ (brs, 1H), $2.94(\mathrm{q}, J=6.7 \mathrm{~Hz}, 2 \mathrm{H})$, $2.55(\mathrm{t}, J=7.5 \mathrm{~Hz}, 2 \mathrm{H}), 1.67-1.54(\mathrm{~m}, 2 \mathrm{H}), 1.54-1.43(\mathrm{~m}, 2 \mathrm{H}) ;{ }^{13} \mathrm{C}$ NMR $\left(101 \mathrm{MHz}, \mathrm{CDCl}_{3}\right) \delta 141.79$, 137.35, 133.82, 131.69, 131.51, 128.51, 128.47, 127.42, 126.04, 43.34, 35.35, 29.17, 28.33; IR (neat): v (cm- 
$\left.{ }^{1}\right) 3309,2929,2859,1578,1329,1159,747,699,666$; HRMS (ESI+) m/z calcd. $\mathrm{C}_{16} \mathrm{H}_{18} \mathrm{ClNNaO}_{2} \mathrm{~S}^{+}[\mathrm{M}+\mathrm{Na}]^{+}$: 346.0639, found: 346.0644 .<smiles>O=[SH](NCCCCc1ccccc1)c1ccc(F)cc1</smiles>

\section{4-Fluoro- $N$-(4-phenylbutyl)benzenesulfonamide (3ae)}

Pale yellow oil; ${ }^{1} \mathrm{H}$ NMR (400 MHz, $\left.\mathrm{CDCl}_{3}\right) \delta 7.90-7.81(\mathrm{~m}, 2 \mathrm{H}), 7.31-7.22(\mathrm{~m}, 2 \mathrm{H}), 7.22-7.12(\mathrm{~m}, 3 \mathrm{H})$, $7.14-7.07(\mathrm{~m}, 2 \mathrm{H}), 4.48(\mathrm{~s}, 1 \mathrm{H}), 2.96(\mathrm{q}, J=6.7 \mathrm{~Hz}, 2 \mathrm{H}), 2.56(\mathrm{t}, J=7.5 \mathrm{~Hz}, 2 \mathrm{H}), 1.66-1.53(\mathrm{~m}, 2 \mathrm{H})$, $1.55-1.42(\mathrm{~m}, 2 \mathrm{H}) ;{ }^{13} \mathrm{C}$ NMR $\left(101 \mathrm{MHz}, \mathrm{CDCl}_{3}\right) \delta 165.16(\mathrm{~d}, J=253.7 \mathrm{~Hz}), 141.77,136.23,129.87(\mathrm{~d}, J=$ $9.4 \mathrm{~Hz}), 128.49(\mathrm{~d}, J=5.0 \mathrm{~Hz}), 126.07,116.46(\mathrm{~d}, J=22.6 \mathrm{~Hz}), 43.23,35.34,29.22,28.31$; IR (neat): $v\left(\mathrm{~cm}^{-}\right.$ $\left.{ }^{1}\right) 3282,2929,2860,1592,1494,1151,1091,837,698$; HRMS (ESI+) m/z calcd. $\mathrm{C}_{16} \mathrm{H}_{19} \mathrm{FNO}_{2} \mathrm{~S}^{+}[\mathrm{M}+\mathrm{H}]^{+}$: 308.1115, found: 308.1114 ;<smiles>O=[SH](NCCCCc1ccccc1)c1ccc(Cl)cc1</smiles>

\section{4-Chloro- $N$-(4-phenylbutyl)benzenesulfonamide (3af)}

White solid, m.p.: $84.4-85.5^{\circ} \mathrm{C} ;{ }^{1} \mathrm{H}$ NMR $\left(400 \mathrm{MHz}, \mathrm{CDCl}_{3}\right) \delta 7.77(\mathrm{~d}, J=8.6 \mathrm{~Hz}, 2 \mathrm{H}), 7.47(\mathrm{~d}, J=8.6 \mathrm{~Hz}$, 2H), $7.30-7.24(\mathrm{~m}, 2 \mathrm{H}), 7.21-7.15(\mathrm{~m}, 1 \mathrm{H}), 7.14-7.06(\mathrm{~m}, 2 \mathrm{H}), 4.48(\mathrm{t}, J=6.2 \mathrm{~Hz}, 1 \mathrm{H}), 2.97(\mathrm{q}, J=6.7$ $\mathrm{Hz}, 2 \mathrm{H}), 2.56(\mathrm{t}, J=7.4 \mathrm{~Hz}, 2 \mathrm{H}), 1.73-1.56(\mathrm{~m}, 2 \mathrm{H}), 1.54-1.42(\mathrm{~m}, 2 \mathrm{H}) ;{ }^{13} \mathrm{C} \mathrm{NMR}\left(101 \mathrm{MHz}, \mathrm{CDCl}_{3}\right) \delta$ $141.75,139.25,138.68,129.54,128.63,128.53,128.46,126.08,43.25,35.33,29.23,28.29 ;$ IR (neat): $v\left(\mathrm{~cm}^{-}\right.$ $\left.{ }^{1}\right) 3269,2921,2857,1586,1324,1157,753,696$; HRMS (ESI+) m/z calcd. $\mathrm{C}_{16} \mathrm{H}_{18} \mathrm{ClNNaO}_{2} \mathrm{~S}^{+}[\mathrm{M}+\mathrm{Na}]^{+}$: 346.0639, found: 346.0643 .<smiles>O=[SH](NCCCCc1ccccc1)c1ccc(Br)cc1</smiles>

\section{4-Bromo- $N$-(4-phenylbutyl)benzenesulfonamide (3ag)}

White solid, m.p.: $102.3-103.2{ }^{\circ} \mathrm{C} ;{ }^{1} \mathrm{H}$ NMR $\left(400 \mathrm{MHz}, \mathrm{CDCl}_{3}\right) \delta 7.70(\mathrm{~d}, J=8.8 \mathrm{~Hz}, 2 \mathrm{H}), 7.64(\mathrm{~d}, J=8.8$ $\mathrm{Hz}, 2 \mathrm{H}), 7.31-7.23(\mathrm{~m}, 2 \mathrm{H}), 7.27-7.14(\mathrm{~m}, 1 \mathrm{H}), 7.14-7.07(\mathrm{~m}, 2 \mathrm{H}), 4.36(\mathrm{t}, J=6.1 \mathrm{~Hz}, 1 \mathrm{H}), 2.97(\mathrm{q}, J=$ $6.7 \mathrm{~Hz}, 2 \mathrm{H}), 2.57(\mathrm{t}, J=7.4 \mathrm{~Hz}, 2 \mathrm{H}), 1.67-1.55(\mathrm{~m}, 2 \mathrm{H}), 1.54-1.41(\mathrm{~m}, 2 \mathrm{H}) ;{ }^{13} \mathrm{C} \mathrm{NMR}\left(101 \mathrm{MHz}, \mathrm{CDCl}_{3}\right)$ 
$\delta 141.74,139.24,132.54,128.74,128.54,128.47,127.71,126.10,43.27,35.34,29.25,28.30$; IR (neat): $v$ $\left(\mathrm{cm}^{-1}\right)$ 3268, 2921, 2855, 1574, 1323, 1155, 740, 697; HRMS (ESI+) m/z calcd. $\mathrm{C}_{16} \mathrm{H}_{19} \mathrm{BrNO}_{2} \mathrm{~S}^{+}[\mathrm{M}+\mathrm{H}]^{+}$: 368.0314, found: 368.0315 .<smiles>Cc1cccc(S(=O)(=O)NCCCCc2ccccc2)c1</smiles>

\section{3-Methyl- $N$-(4-phenylbutyl)benzenesulfonamide (3ah)}

Yellow oil; ${ }^{1} \mathrm{H}$ NMR $\left(400 \mathrm{MHz}, \mathrm{CDCl}_{3}\right) \delta 7.72-7.59(\mathrm{~m}, 2 \mathrm{H}), 7.45-7.34(\mathrm{~m}, 2 \mathrm{H}), 7.29-7.22(\mathrm{~m}, 2 \mathrm{H})$, $7.20-7.14(\mathrm{~m}, 1 \mathrm{H}), 7.14-7.07(\mathrm{~m}, 2 \mathrm{H}), 4.40(\mathrm{brs}, 1 \mathrm{H}), 2.96(\mathrm{q}, J=6.7 \mathrm{~Hz}, 2 \mathrm{H}), 2.55(\mathrm{t}, J=7.5 \mathrm{~Hz}, 2 \mathrm{H})$, $2.41(\mathrm{~s}, 3 \mathrm{H}), 1.67-1.55(\mathrm{~m}, 2 \mathrm{H}), 1.55-1.41(\mathrm{~m}, 2 \mathrm{H}) ;{ }^{13} \mathrm{C} \mathrm{NMR}\left(101 \mathrm{MHz}, \mathrm{CDCl}_{3}\right) \delta 141.89,139.87$, 139.46, 133.54, 129.09, 128.48, 127.52, 126.02, 124.29, 43.23, 35.38, 29.27, 28.36, 21.48; IR (neat): $v\left(\mathrm{~cm}^{-1}\right)$ 3280, 2927, 2859, 1453, 1323, 1150, 689; HRMS (ESI+) m/z calcd. $\mathrm{C}_{17} \mathrm{H}_{22} \mathrm{NO}_{2} \mathrm{~S}^{+}[\mathrm{M}+\mathrm{H}]^{+}:$304.1366, found: 304.1360 .<smiles>CC(=O)Nc1ccc(S(=O)(=O)NCCCCc2ccccc2)cc1</smiles>

$N$-(4-(N-(4-Phenylbutyl)sulfamoyl)phenyl)acetamide (3ai)

Yellow oil; ${ }^{1} \mathrm{H}$ NMR (400 MHz, $\left.\mathrm{CDCl}_{3}\right) \delta 7.96$ (brs, $\left.1 \mathrm{H}\right), 7.71(\mathrm{~d}, J=8.4 \mathrm{~Hz}, 2 \mathrm{H}), 7.61(\mathrm{~d}, J=8.5 \mathrm{~Hz}, 2 \mathrm{H})$, $7.28-7.20(\mathrm{~m}, 2 \mathrm{H}), 7.16(\mathrm{t}, J=7.3 \mathrm{~Hz}, 1 \mathrm{H}), 7.10(\mathrm{~d}, J=7.4 \mathrm{~Hz}, 2 \mathrm{H}), 4.79(\mathrm{t}, J=6.2 \mathrm{~Hz}, 1 \mathrm{H}), 2.92(\mathrm{q}, J=$ $6.5 \mathrm{~Hz}, 2 \mathrm{H}), 2.54(\mathrm{t}, J=7.5 \mathrm{~Hz}, 2 \mathrm{H}), 2.19(\mathrm{~s}, 3 \mathrm{H}), 1.64-1.52(\mathrm{~m}, 2 \mathrm{H}), 1.54-1.42(\mathrm{~m}, 2 \mathrm{H}) ;{ }^{13} \mathrm{C} \mathrm{NMR}(101$ $\left.\mathrm{MHz}, \mathrm{CDCl}_{3}\right) \delta 169.14,142.06,141.88,134.65,128.48,128.34,126.00,119.61,43.22,35.35,29.22,28.33$, 24.81; IR (neat): $v\left(\mathrm{~cm}^{-1}\right) 3289,2954,2845,1733,1415,1355,1150,699 ;$ HRMS (ESI+) m/z calcd. $\mathrm{C}_{18} \mathrm{H}_{23} \mathrm{~N}_{2} \mathrm{O}_{3} \mathrm{~S}^{+}[\mathrm{M}+\mathrm{H}]^{+}:$347.1424, found: 347.1429 .<smiles>CC(C)Cc1ccc(S(=O)(=O)NCCCCc2ccccc2)cc1</smiles>

\section{4-(Tert-butyl)- $N$-(4-phenylbutyl)benzenesulfonamide (3aj)}

Colorless oil; ${ }^{1} \mathrm{H}$ NMR (400 MHz, $\left.\mathrm{CDCl}_{3}\right) \delta 7.77(\mathrm{~d}, J=8.6 \mathrm{~Hz}, 2 \mathrm{H}), 7.51(\mathrm{~d}, J=8.6 \mathrm{~Hz}, 2 \mathrm{H}), 7.30-7.21$ $(\mathrm{m}, 2 \mathrm{H}), 7.21-7.14(\mathrm{~m}, 1 \mathrm{H}), 7.13-7.07(\mathrm{~m}, 2 \mathrm{H}), 4.46(\mathrm{t}, J=6.4 \mathrm{~Hz}, 1 \mathrm{H}), 2.96(\mathrm{q}, J=6.7 \mathrm{~Hz}, 2 \mathrm{H}), 2.55(\mathrm{t}$, $J=7.5 \mathrm{~Hz}, 2 \mathrm{H}), 1.66-1.56(\mathrm{~m}, 2 \mathrm{H}), 1.56-1.44(\mathrm{~m}, 2 \mathrm{H}), 1.34(\mathrm{~m}, 9 \mathrm{H}) ;{ }^{13} \mathrm{C} \mathrm{NMR}\left(101 \mathrm{MHz}, \mathrm{CDCl}_{3}\right) \delta$ 156.50, 141.92, 136.99, 128.48, 127.04, 126.20, 126.00, 43.20, 35.38, 35.26, 31.22, 29.26, 28.34; IR (neat): 
$v\left(\mathrm{~cm}^{-1}\right)$ 3278, 2962, 2866, 1323, 1159, 627; HRMS (ESI+) m/z calcd. $\mathrm{C}_{20} \mathrm{H}_{28} \mathrm{NO}_{2} \mathrm{~S}^{+}[\mathrm{M}+\mathrm{H}]^{+}: 346.1835$, found: 346.1838 .<smiles>O=[N+](NCCCCc1ccccc1)c1ccc2ccccc2c1</smiles>

\section{$N$-(4-phenylbutyl)naphthalene-2-sulfonamide (3ak)}

Colorless oil; ${ }^{1} \mathrm{H}$ NMR $\left(400 \mathrm{MHz}, \mathrm{CDCl}_{3}\right) \delta 8.43(\mathrm{~d}, J=2.0 \mathrm{~Hz}, 1 \mathrm{H}), 8.03-7.88(\mathrm{~m}, 3 \mathrm{H}), 7.81(\mathrm{dd}, J=8.7$, $1.9 \mathrm{~Hz}, 1 \mathrm{H}), 7.71-7.56(\mathrm{~m}, 2 \mathrm{H}), 7.26-7.17(\mathrm{~m}, 2 \mathrm{H}), 7.17-7.11(\mathrm{~m}, 1 \mathrm{H}), 7.11-7.02(\mathrm{~m}, 2 \mathrm{H}), 4.43(\mathrm{t}, J=$ $6.2 \mathrm{~Hz}, 1 \mathrm{H}), 3.00(\mathrm{q}, J=6.7 \mathrm{~Hz}, 2 \mathrm{H}), 2.53(\mathrm{t}, J=7.4 \mathrm{~Hz}, 2 \mathrm{H}), 1.63-1.55(\mathrm{~m}, 2 \mathrm{H}), 1.53-1.45(\mathrm{~m}, 2 \mathrm{H}) ;{ }^{13} \mathrm{C}$ NMR $\left(101 \mathrm{MHz}, \mathrm{CDCl}_{3}\right) \delta 141.81,136.88,134.95,132.31,129.65,129.38,128.93,128.57,128.47,128.44$, 128.07, 127.72, 126.01, 122.45, 43.29, 35.35, 29.30, 28.33; IR (neat): $v\left(\mathrm{~cm}^{-1}\right)$ 3247, 2921, 2853, 1632, 1312 , 1150, 697; HRMS (ESI+) m/z calcd. $\mathrm{C}_{20} \mathrm{H}_{22} \mathrm{NO}_{2} \mathrm{~S}^{+}[\mathrm{M}+\mathrm{H}]^{+}: 340.1366$, found: 340.1360 .<smiles>O=S(NCCCCc1ccccc1)c1cccs1</smiles>

\section{$N$-(4-phenylbutyl)thiophene-2-sulfonamide (3al)}

Yellow oil; ${ }^{1} \mathrm{H}$ NMR $\left(400 \mathrm{MHz}, \mathrm{CDCl}_{3}\right) \delta 7.62-7.54(\mathrm{~m}, 2 \mathrm{H}), 7.31-7.21(\mathrm{~m}, 2 \mathrm{H}), 7.22-7.13(\mathrm{~m}, 1 \mathrm{H})$, $7.16-7.09(\mathrm{~m}, 2 \mathrm{H}), 7.07(\mathrm{dd}, J=5.0,3.7 \mathrm{~Hz}, 1 \mathrm{H}), 4.53(\mathrm{t}, J=6.2 \mathrm{~Hz}, 1 \mathrm{H}), 3.05(\mathrm{q}, J=6.9 \mathrm{~Hz}, 2 \mathrm{H}), 2.58(\mathrm{t}$, $J=7.5 \mathrm{~Hz}, 2 \mathrm{H}), 1.69-1.58(\mathrm{~m}, 2 \mathrm{H}), 1.59-1.46(\mathrm{~m}, 2 \mathrm{H}) ;{ }^{13} \mathrm{C} \mathrm{NMR}\left(101 \mathrm{MHz}, \mathrm{CDCl}_{3}\right) \delta 141.84,141.09$, 132.21, 131.89, 128.51, 127.50, 126.04, 43.48, 35.36, 29.10, 28.34; IR (neat): $v$ (cm $\left.{ }^{-1}\right) 3283,2926,2859$, 1405, 1325, 1150, 699; HRMS (ESI+) m/z calcd. $\mathrm{C}_{14} \mathrm{H}_{18} \mathrm{NO}_{2} \mathrm{~S}_{2}{ }^{+}[\mathrm{M}+\mathrm{H}]+:$ 296.0773, found: 296.0779.<smiles>O=C(OCc1ccccc1)[I-]NCCCCc1ccccc1</smiles>

2,2,2-trichloroethyl (4-phenylbutyl)carbamate (7aa)

Pale yellow oil; ${ }^{1} \mathrm{H}$ NMR (400 MHz, $\left.\mathrm{CDCl}_{3}\right) \delta 7.32$ - $7.23(\mathrm{~m}, 2 \mathrm{H}), 7.23-7.12(\mathrm{~m}, 3 \mathrm{H}), 4.94(\mathrm{~s}, 1 \mathrm{H}), 4.71$ (s, 2H), $3.25(\mathrm{td}, J=6.9,6.0 \mathrm{~Hz}, 2 \mathrm{H}), 2.64(\mathrm{t}, J=7.5 \mathrm{~Hz}, 2 \mathrm{H}), 1.73-1.61(\mathrm{~m}, 2 \mathrm{H}), 1.61-1.53(\mathrm{~m}, 2 \mathrm{H}) ;{ }^{13} \mathrm{C}$ NMR $\left(101 \mathrm{MHz}, \mathrm{CDCl}_{3}\right) \delta 154.57,141.96,128.40,125.90,95.70,74.49,41.15,35.44,29.38,28.43 ; \mathrm{IR}$ (neat): $v\left(\mathrm{~cm}^{-1}\right)$ 3352, 2958, 2920, 2855, 1726, 1575, 1241, 1160, 813, 724; HRMS (ESI+) m/z calcd. $\mathrm{C}_{13} \mathrm{H}_{16} \mathrm{Cl}_{3} \mathrm{NNaO}_{2}^{+}[\mathrm{M}+\mathrm{Na}]^{+}:$346.01339, found: 346.0142 .<smiles>O=C([NH2+]CCCc1ccccc1)OCc1ccccc1</smiles> 
Pale yellow oil; ${ }^{1} \mathrm{H}$ NMR (400 MHz, $\left.\mathrm{CDCl}_{3}\right) \delta 7.34-7.23(\mathrm{~m}, 2 \mathrm{H}), 7.23-7.14(\mathrm{~m}, 3 \mathrm{H}), 4.98(\mathrm{~s}, 1 \mathrm{H}), 4.72$ $(\mathrm{s}, 2 \mathrm{H}), 3.27(\mathrm{td}, J=7.1,6.0 \mathrm{~Hz}, 2 \mathrm{H}), 2.71-2.63(\mathrm{~m}, 2 \mathrm{H}), 1.94-1.82(\mathrm{~m}, 2 \mathrm{H}) ;{ }^{13} \mathrm{C} \mathrm{NMR}\left(101 \mathrm{MHz}, \mathrm{CDCl}_{3}\right)$ $\delta 154.57,141.15,128.53,128.36,126.11,95.70,74.49,40.88,32.97,31.37$; IR (neat): $v\left(\mathrm{~cm}^{-1}\right) 3492,2960$, 2912, 1705, 1578, 1080, 833, 698; HRMS (ESI+) m/z calcd. $\mathrm{C}_{12} \mathrm{H}_{14} \mathrm{Cl}_{3} \mathrm{NNaO}_{2}{ }^{+}[\mathrm{M}+\mathrm{Na}]^{+}:$331.9982, found: 331.9988.<smiles>O=C(OCc1ccccc1)[I-][NH2+]Cc1ccccc1</smiles>

\section{2,2,2-trichloroethyl benzylcarbamate (7ca)}

Pale yellow oil; ${ }^{1} \mathrm{H}$ NMR (400 MHz, $\left.\mathrm{CDCl}_{3}\right) \delta 7.41-7.23(\mathrm{~m}, 5 \mathrm{H}), 5.33(\mathrm{~s}, 1 \mathrm{H}), 4.76(\mathrm{~s}, 2 \mathrm{H}), 4.42(\mathrm{~d}, J=$ $6.0 \mathrm{~Hz}, 2 \mathrm{H}) ;{ }^{13} \mathrm{C}$ NMR $(101 \mathrm{MHz}, \mathrm{CDCl} 3) \delta 154.65,137.75,128.80,127.78,127.58,95.59,74.65,45.35 ; \mathrm{IR}$ (neat): $v\left(\mathrm{~cm}^{-1}\right) 3355,2958,2920,1526,1158,1067,816,723,666 ;$ HRMS (ESI+) $\mathrm{m} / \mathrm{z}$ calcd. $\mathrm{C}_{10} \mathrm{H}_{10} \mathrm{Cl}_{3} \mathrm{NNaO}_{2}^{+}[\mathrm{M}+\mathrm{Na}]^{+}:$303.9669, found: 303.9672 .<smiles>CC(Cc1ccccc1)N[In]C(=O)O</smiles>

2,2,2-trichloroethyl (1-phenylpropan-2-yl)carbamate (7da)

Pale yellow oil; ${ }^{1} \mathrm{H}$ NMR (400 MHz, CDCl3) $\delta 7.49-6.88(\mathrm{~m}, 5 \mathrm{H}), 4.81(\mathrm{~s}, 1 \mathrm{H}), 4.74-4.66$ (m, 2H), 4.08 $-3.97(\mathrm{~m}, 1 \mathrm{H}), 2.87(\mathrm{dd}, J=13.5,6.1 \mathrm{~Hz}, 1 \mathrm{H}), 2.75(\mathrm{dd}, J=13.6,7.0 \mathrm{~Hz}, 1 \mathrm{H}), 1.17(\mathrm{~d}, J=6.6 \mathrm{~Hz}, 3 \mathrm{H}) ;{ }^{13} \mathrm{C}$ NMR (101 MHz, CDCl3) $\delta 153.73,137.59,129.48,128.47,126.59,95.70,74.36,48.43,42.68,20.15 ;$ IR (neat): $v\left(\mathrm{~cm}^{-1}\right) 3312,2945,1718,1588,1218,1130,1069,766$; HRMS (ESI+) m/z calcd. $\mathrm{C}_{12} \mathrm{H}_{14} \mathrm{Cl}_{3} \mathrm{NNaO}_{2}{ }^{+}$ $[\mathrm{M}+\mathrm{Na}]^{+}:$331.9982, found: 331.9974 .

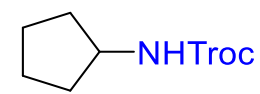

\section{2,2,2-trichloroethyl cyclopentylcarbamate (7ea)}

Pale yellow oil; ${ }^{1} \mathrm{H}$ NMR (400 MHz, $\left.\mathrm{CDCl}_{3}\right) \delta 4.93(\mathrm{~s}, 1 \mathrm{H}), 4.71(\mathrm{~s}, 2 \mathrm{H}), 4.08-3.94(\mathrm{~m}, 1 \mathrm{H}), 2.06-1.94$ $(\mathrm{m}, 2 \mathrm{H}), 1.79-1.54(\mathrm{~m}, 4 \mathrm{H}), 1.53-1.39(\mathrm{~m}, 2 \mathrm{H}) ;{ }^{13} \mathrm{C} \mathrm{NMR}\left(101 \mathrm{MHz}, \mathrm{CDCl}_{3}\right) \delta 153.98,95.72,74.45$, 53.11, 33.11, 23.99; IR (neat): $v\left(\mathrm{~cm}^{-1}\right)$ 3275, 2955, 2856, 1720, 1451, 1157, 663; HRMS (ESI+) m/z calcd. $\mathrm{C}_{8} \mathrm{H}_{11} \mathrm{Cl}_{3} \mathrm{NO}_{2}^{+}[\mathrm{M}-\mathrm{H}]^{+}:$257.9855, found: 257.9860 .<smiles>O=[Po]C1CCCCC1</smiles> 
White soild, M.p. $=92-94{ }^{\circ} \mathrm{C} ;{ }^{1} \mathrm{H}$ NMR $\left(400 \mathrm{MHz}, \mathrm{CDCl}_{3}\right) \delta 4.88(\mathrm{~s}, 1 \mathrm{H}), 4.73(\mathrm{~s}, 2 \mathrm{H}), 3.61-3.47$ (m, 1H), $2.04-1.94(\mathrm{~m}, 2 \mathrm{H}), 1.81-1.69(\mathrm{~m}, 2 \mathrm{H}), 1.69-1.58(\mathrm{~m}, 1 \mathrm{H}), 1.44-1.32(\mathrm{~m}, 2 \mathrm{H}), 1.27-1.12(\mathrm{~m}, 3 \mathrm{H})$; ${ }^{13} \mathrm{C} \mathrm{NMR}\left(101 \mathrm{MHz}, \mathrm{CDCl}_{3}\right) \delta 153.67,95.77,74.41,50.30,33.19,25.42,24.74$. IR (neat): $v\left(\mathrm{~cm}^{-1}\right) 3328$, 2945, 2865, 1726, 1544, 1302, 1058, 730, 699; HRMS (ESI+) m/z calcd. $\mathrm{C}_{9} \mathrm{H}_{13} \mathrm{Cl}_{3} \mathrm{NO}_{2}{ }^{+}[\mathrm{M}-\mathrm{H}]^{+}: 272.0012$, found: 272.0008 .<smiles>CCOC(=O)CC(C)N[O+]</smiles>

ethyl 3-(((2,2,2-trichloroethoxy)carbonyl)amino)butanoate (7ga)

Pale yellow oil; ${ }^{1} \mathrm{H}$ NMR (400 MHz, $\left.\mathrm{CDCl}_{3}\right) \delta 5.49(\mathrm{~s}, 1 \mathrm{H}), 4.82-4.64(\mathrm{~m}, 2 \mathrm{H}), 4.26-4.06(\mathrm{~m}, 3 \mathrm{H}), 2.56$ $(\mathrm{d}, J=5.6 \mathrm{~Hz}, 0 \mathrm{H}), 1.34-1.23(\mathrm{~m}, 6 \mathrm{H}) ;{ }^{13} \mathrm{C} \mathrm{NMR}\left(101 \mathrm{MHz}, \mathrm{CDCl}_{3}\right) \delta 171.30,153.73,95.62,74.46,60.72$, 44.38, 40.23, 20.24, 14.20; IR (neat): $v\left(\mathrm{~cm}^{-1}\right) 3277,2981,2844,1730,1463,1303,1087,666 ;$ HRMS (ESI+) m/z calcd. $\mathrm{C}_{9} \mathrm{H}_{14} \mathrm{Cl}_{3} \mathrm{NNaO}_{4}{ }^{+}[\mathrm{M}+\mathrm{Na}]^{+}: 327.9881$, found: 327.9879 .<smiles>O=C(NCCCCc1ccccc1)OCCCl</smiles>

2-chloroethyl (4-phenylbutyl)carbamate (7ab)

Pale yellow oil; ${ }^{1} \mathrm{H}$ NMR (400 MHz, $\left.\mathrm{CDCl}_{3}\right) \delta 7.33-7.24(\mathrm{~m}, 3 \mathrm{H}), 7.23-7.12(\mathrm{~m}, 4 \mathrm{H}), 4.75(\mathrm{~s}, 1 \mathrm{H}), 4.30(\mathrm{t}$, $J=5.6 \mathrm{~Hz}, 2 \mathrm{H}), 3.66(\mathrm{t}, J=5.6 \mathrm{~Hz}, 3 \mathrm{H}), 3.20(\mathrm{q}, J=6.7 \mathrm{~Hz}, 2 \mathrm{H}), 2.63(\mathrm{t}, J=7.5 \mathrm{~Hz}, 2 \mathrm{H}), 1.70-1.61(\mathrm{~m}$, $2 \mathrm{H}), 1.58-1.50(\mathrm{~m}, 2 \mathrm{H}) ;{ }^{13} \mathrm{C} \mathrm{NMR}\left(101 \mathrm{MHz}, \mathrm{CDCl}_{3}\right) \delta 155.88,142.04,128.40,128.37,125.86,64.42$, 42.33, 40.94, 35.47, 29.50, 28.49; IR (neat): $v\left(\mathrm{~cm}^{-1}\right) 3338,3036,2943,2806,1712,1599,1447,1313,762$, 696; HRMS (ESI+) m/z calcd. $\mathrm{C}_{13} \mathrm{H}_{18} \mathrm{ClNNaO}_{2}{ }^{+}[\mathrm{M}+\mathrm{Na}]^{+}: 278.0918$, found: 278.0912 .<smiles>CCCCCOC(=O)NCCCCc1ccccc1</smiles>

pentyl (4-phenylbutyl)carbamate (7ac)

Pale yellow oil; ${ }^{1} \mathrm{H}$ NMR $\left(400 \mathrm{MHz}, \mathrm{CDCl}_{3}\right) \delta 7.32-7.23(\mathrm{~m}, 2 \mathrm{H}), 7.22-7.12(\mathrm{~m}, 3 \mathrm{H}), 4.61(\mathrm{~s}, 1 \mathrm{H}), 4.03(\mathrm{t}$, $J=6.7 \mathrm{~Hz}, 2 \mathrm{H}), 3.19(\mathrm{q}, J=6.7 \mathrm{~Hz}, 2 \mathrm{H}), 2.63(\mathrm{t}, J=7.6 \mathrm{~Hz}, 2 \mathrm{H}), 1.69-1.57(\mathrm{~m}, 4 \mathrm{H}), 1.57-1.49(\mathrm{~m}, 2 \mathrm{H})$, $1.42-1.27(\mathrm{~m}, 2 \mathrm{H}), 0.90(\mathrm{t}, \mathrm{J}=6.7 \mathrm{~Hz} 1 \mathrm{H}) ; 13 \mathrm{C} \mathrm{NMR}(101 \mathrm{MHz}, \mathrm{CDCl} 3) \delta 156.81,142.13,128.39,128.33$, 125.81, 64.93, 40.81, 35.50, 29.72, 29.66, 28.76, 28.52, 28.04, 22.37, 13.99; IR (neat): v (cm $\left.{ }^{-1}\right) 3396,2912$, 2875, 1720, 1568, 1342, 1057, 753, 696; HRMS (ESI+) m/z calcd. $\mathrm{C}_{16} \mathrm{H}_{25} \mathrm{NNaO}_{2}{ }^{+}[\mathrm{M}+\mathrm{Na}]^{+}:$286.1778, found: 286.1772 . 
<smiles>O=C(NCCCCc1ccccc1)Oc1ccccc1</smiles>

phenyl (4-phenylbutyl)carbamate (7ad)

Pale yellow oil; ${ }^{1} \mathrm{H}$ NMR (400 MHz, $\left.\mathrm{CDCl}_{3}\right) \delta 7.39-7.23(\mathrm{~m}, 4 \mathrm{H}), 7.24-7.14(\mathrm{~m}, 4 \mathrm{H}), 7.14-7.08(\mathrm{~m}, 2 \mathrm{H})$, 4.97 (s, 1H), 3.29 (q, $J=6.7 \mathrm{~Hz}, 2 \mathrm{H}), 2.66(\mathrm{t}, J=7.5 \mathrm{~Hz}, 2 \mathrm{H}), 1.77-1.66(\mathrm{~m}, 2 \mathrm{H}), 1.66-1.57(\mathrm{~m}, 2 \mathrm{H}) ;{ }^{13} \mathrm{C}$ NMR (101 MHz, $\left.\mathrm{CDCl}_{3}\right) \delta 154.59,151.08,142.02,129.27,128.41,128.38,125.88,125.22,121.58,41.10$, 35.46, 29.43, 28.50; IR (neat): $v\left(\mathrm{~cm}^{-1}\right)$ 3346, 2985, 2866 1498, 1162, 973, 666, 545; HRMS (ESI+) m/z calcd. $\mathrm{C}_{17} \mathrm{H}_{19} \mathrm{NNaO}_{2}{ }^{+}[\mathrm{M}+\mathrm{Na}]^{+}: 292.1308$, found: 292.1307 .<smiles>CC(C)(C)OC(=O)NCCCc1ccccc1</smiles>

tert-butyl (3-phenylpropyl)carbamate (7be)

Pale yellow oil; ${ }^{1} \mathrm{H}$ NMR (400 MHz, $\left.\mathrm{CDCl}_{3}\right) \delta 7.31-7.23$ (m, 2H), $7.21-7.14(\mathrm{~m}, 3 \mathrm{H}), 4.56$ (s, 1H), 3.15 (q, $J=6.9 \mathrm{~Hz}, 2 \mathrm{H}), 2.63(\mathrm{t}, J=7.6 \mathrm{~Hz}, 2 \mathrm{H}), 1.86-1.74(\mathrm{~m}, 2 \mathrm{H}), 1.44(\mathrm{~s}, 9 \mathrm{H}) ; 13 \mathrm{C}$ NMR $(101 \mathrm{MHz}, \mathrm{CDCl} 3)$ $\delta$ 156.00, 141.58, 128.43, 128.37, 125.93, 79.12, 40.26, 33.14, 31.76, 28.44; IR (neat): $v\left(\mathrm{~cm}^{-1}\right) 3287,2962$, 2866, 1548, 1323, 1159, 1099, 627; HRMS (ESI+) m/z calcd. $\mathrm{C}_{14} \mathrm{H}_{21} \mathrm{NNaO}_{2}{ }^{+}[\mathrm{M}+\mathrm{Na}]^{+}: 258.1465$, found: 258.1458. 


\section{8. ${ }^{1} \mathrm{H}$ and ${ }^{13} \mathrm{C}$ NMR Spectra}

\section{Compound 3aa}

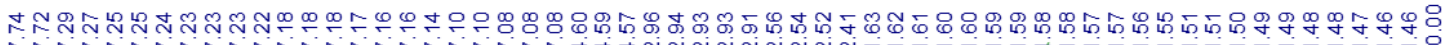

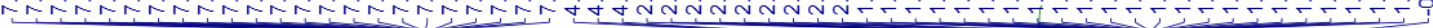
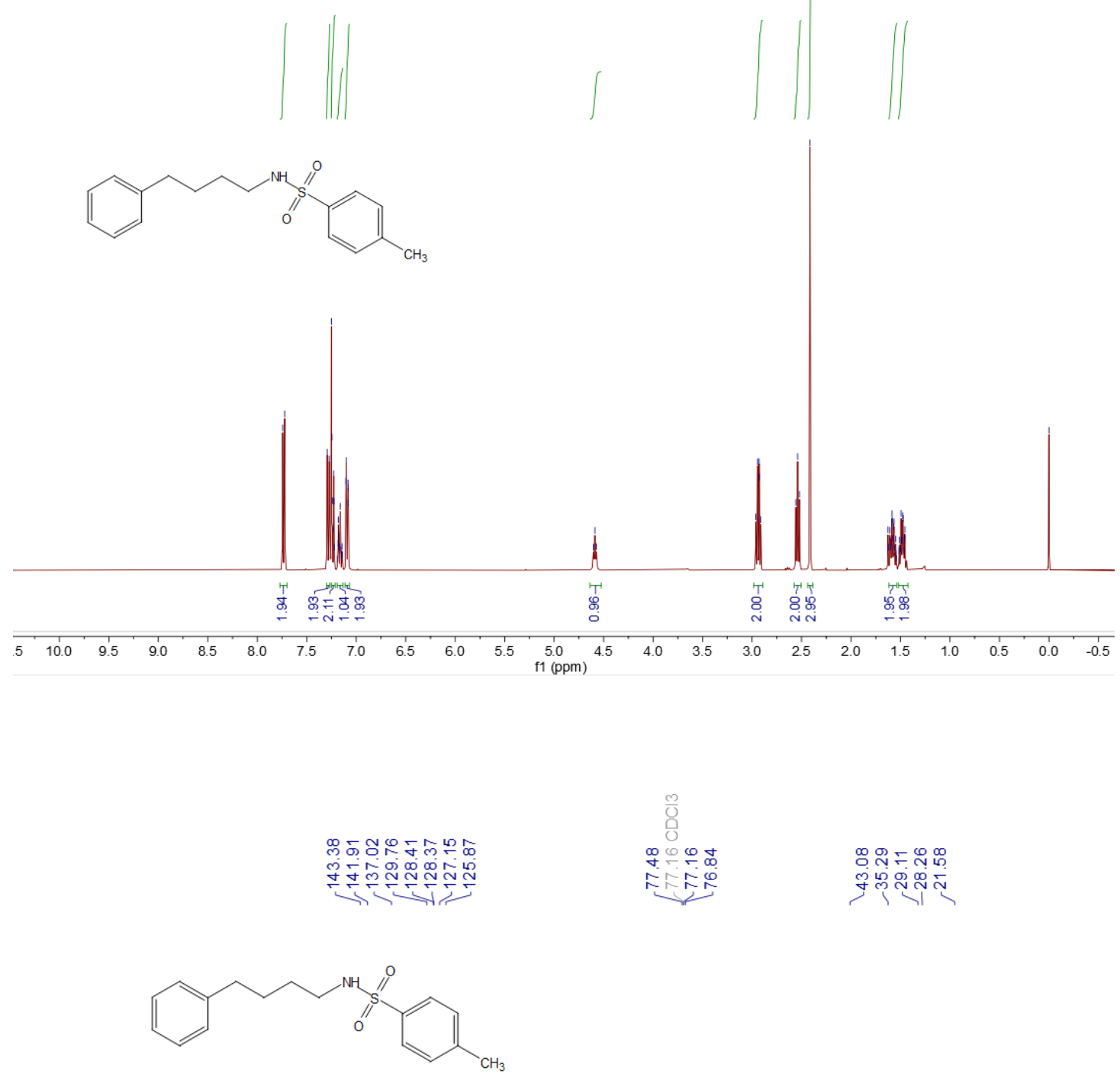

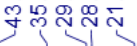

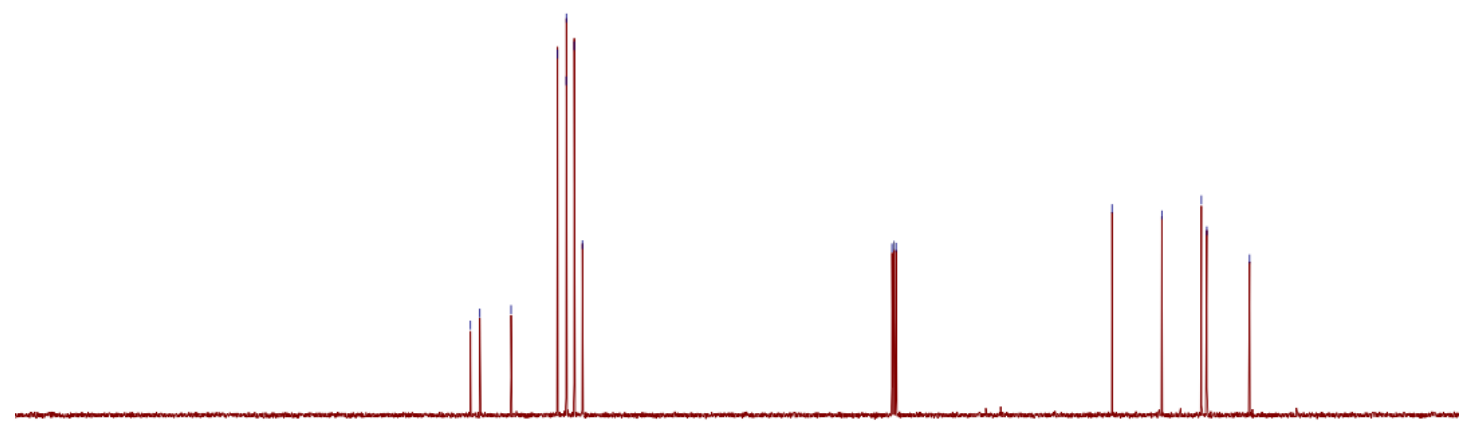

$\begin{array}{lllllllllllllllllllllllllllllllll}1 & 1 & 200 & 190 & 180 & 170 & 160 & 150 & 140 & 130 & 120 & 110 & 100 & 90 & 80 & 70 & 60 & 50 & 40 & 30 & 20 & 10 & 0 & -11\end{array}$ 


\section{Compound 3ba}

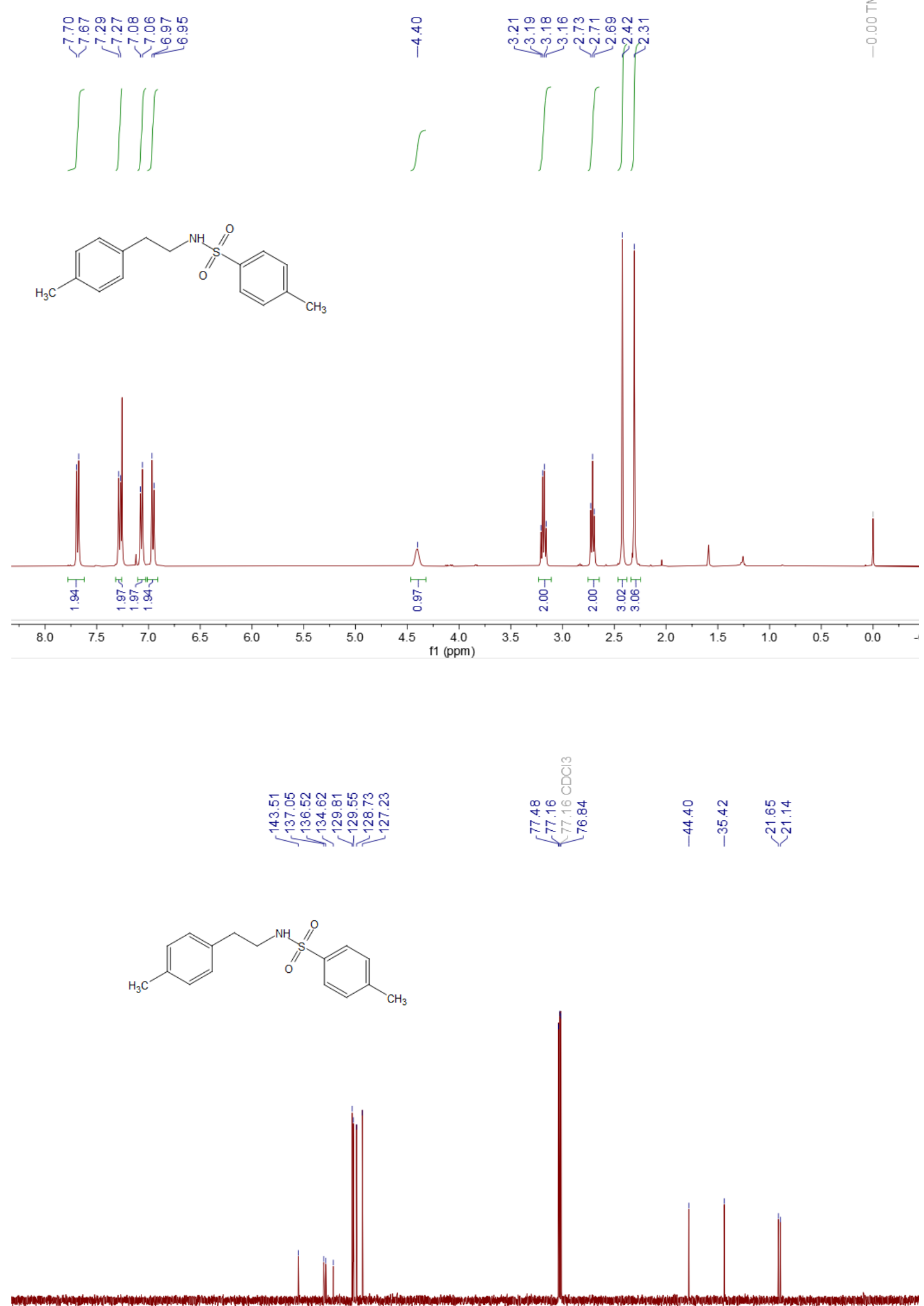

\begin{tabular}{lllllllllllllllllllllllll}
\hline & 210 & 200 & 190 & 180 & 170 & 160 & 150 & 140 & 130 & 120 & 110 & 100 & 90 & 80 & 70 & 60 & 50 & 40 & 30 & 20 & 10 & 0 & -10 \\
$\mathrm{f} 1(\mathrm{ppm})$
\end{tabular} 
Compound 3ca

$\substack{0 \\ \vdots \\ 0 \\ 0 \\ i}$
$i$

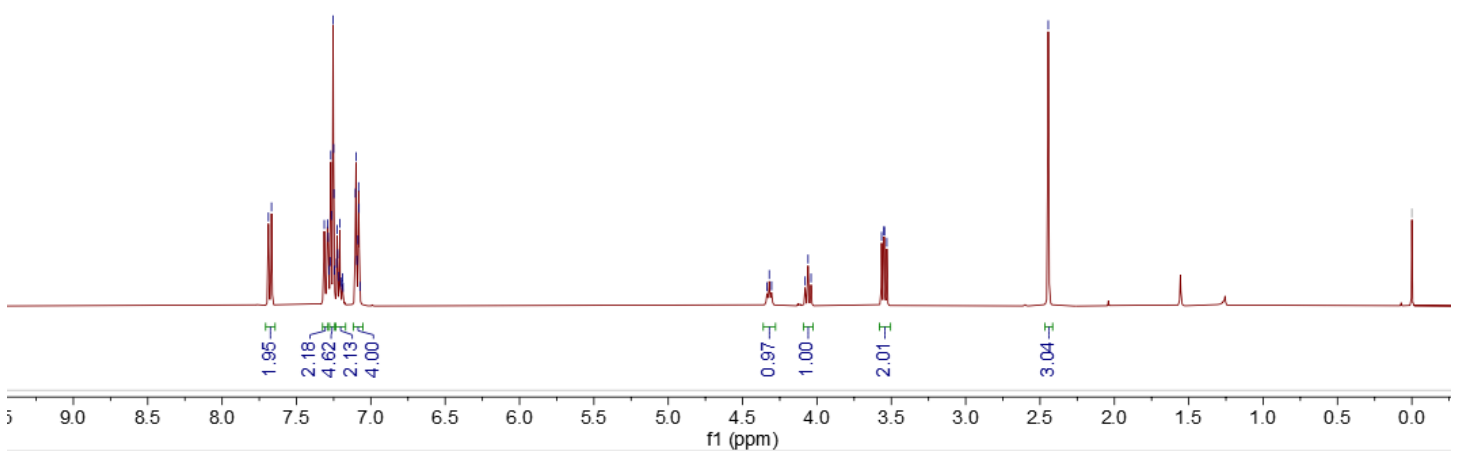

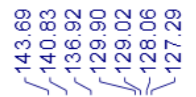

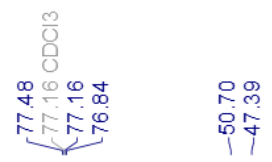

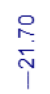
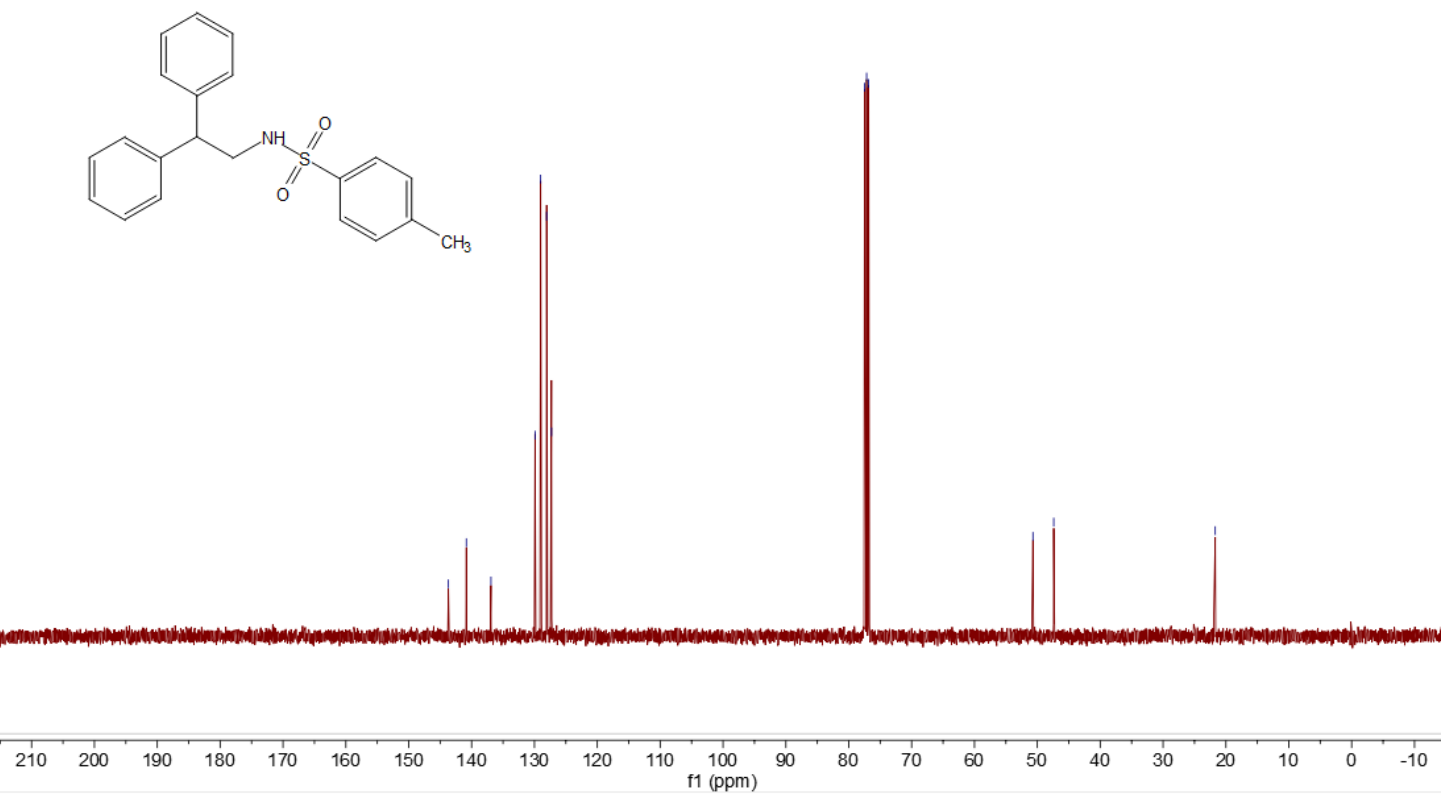

S42 
Compound 3da

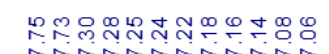
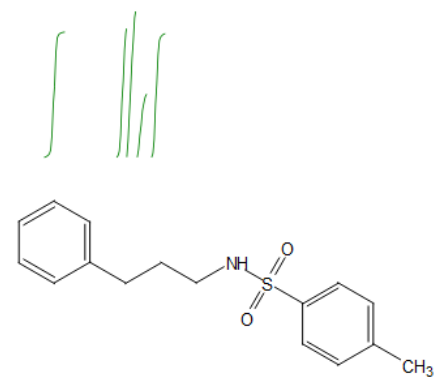

匀

$\int$

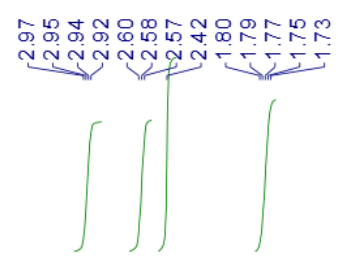

$\infty$
$\sum$
0
0
0
1

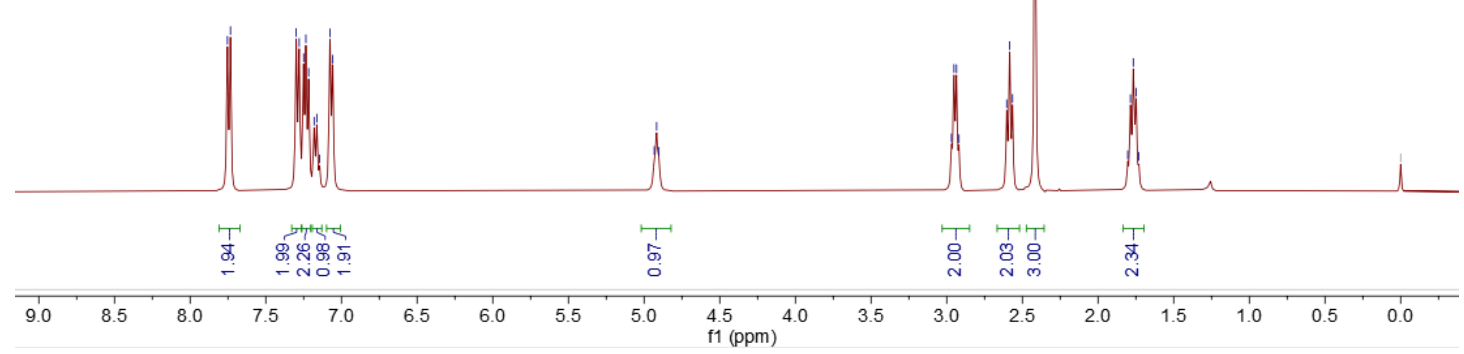

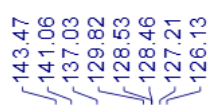
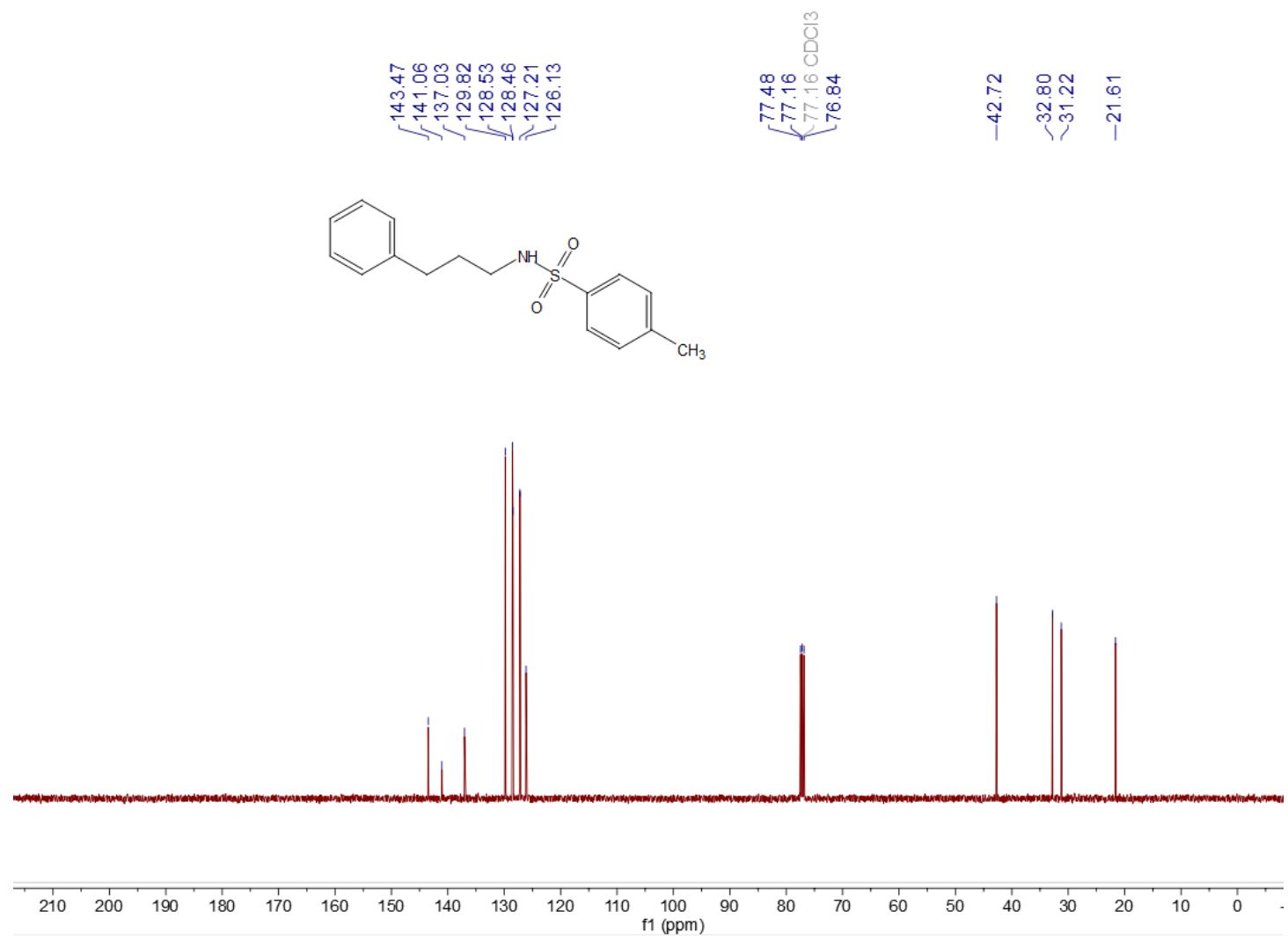

S43 


\section{Compound 3ea}

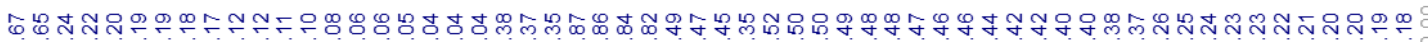<smiles>C1#CC2CCC1C2</smiles><smiles>Cc1ccc(S(=O)(=O)NCCCCCc2ccccc2)cc1</smiles>
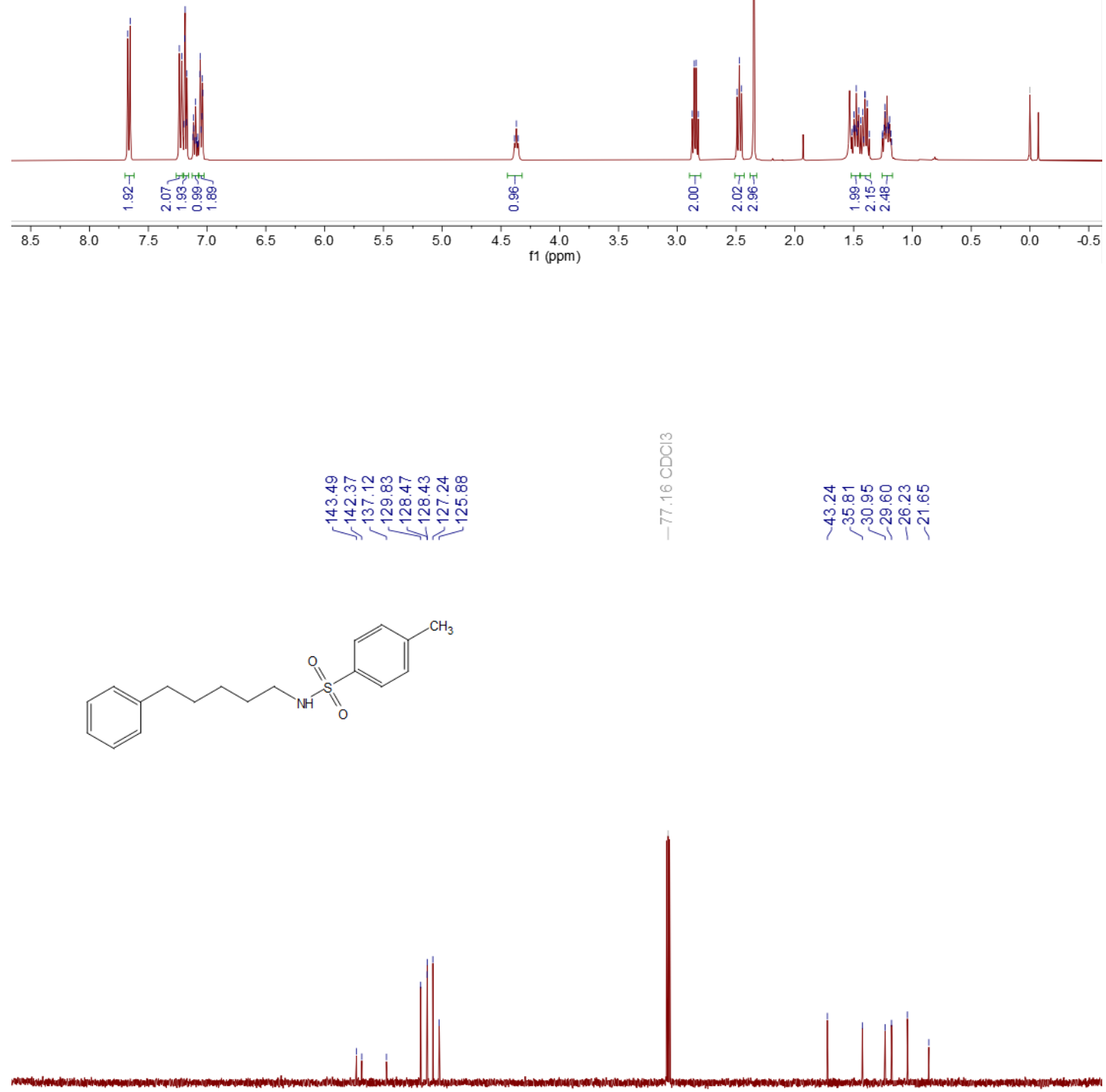

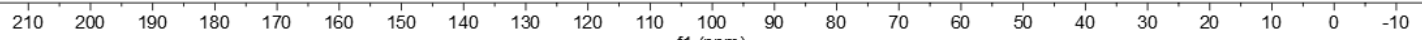


Compound 3fa
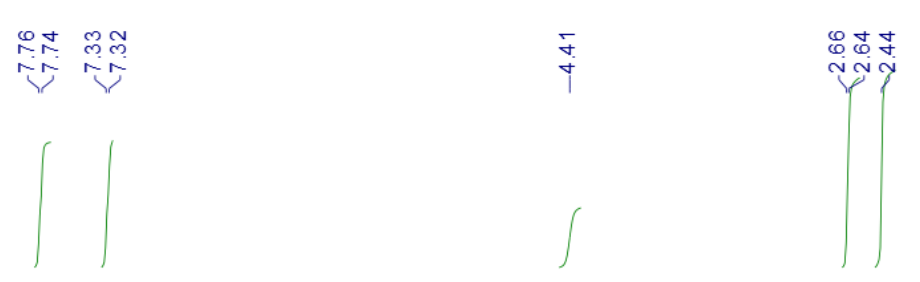

$\sum_{j}^{0}$
$\vdots$
0
0
$i$
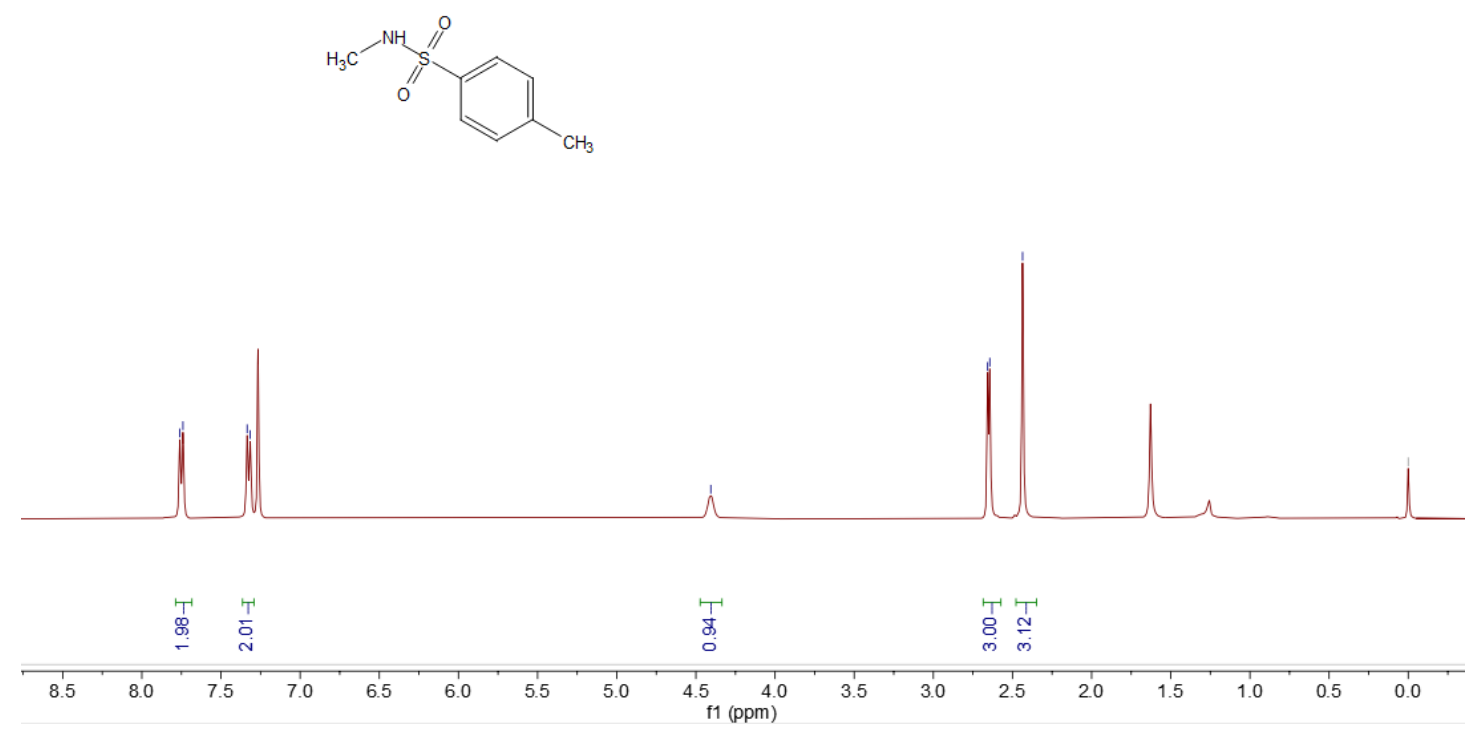

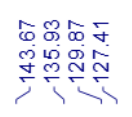
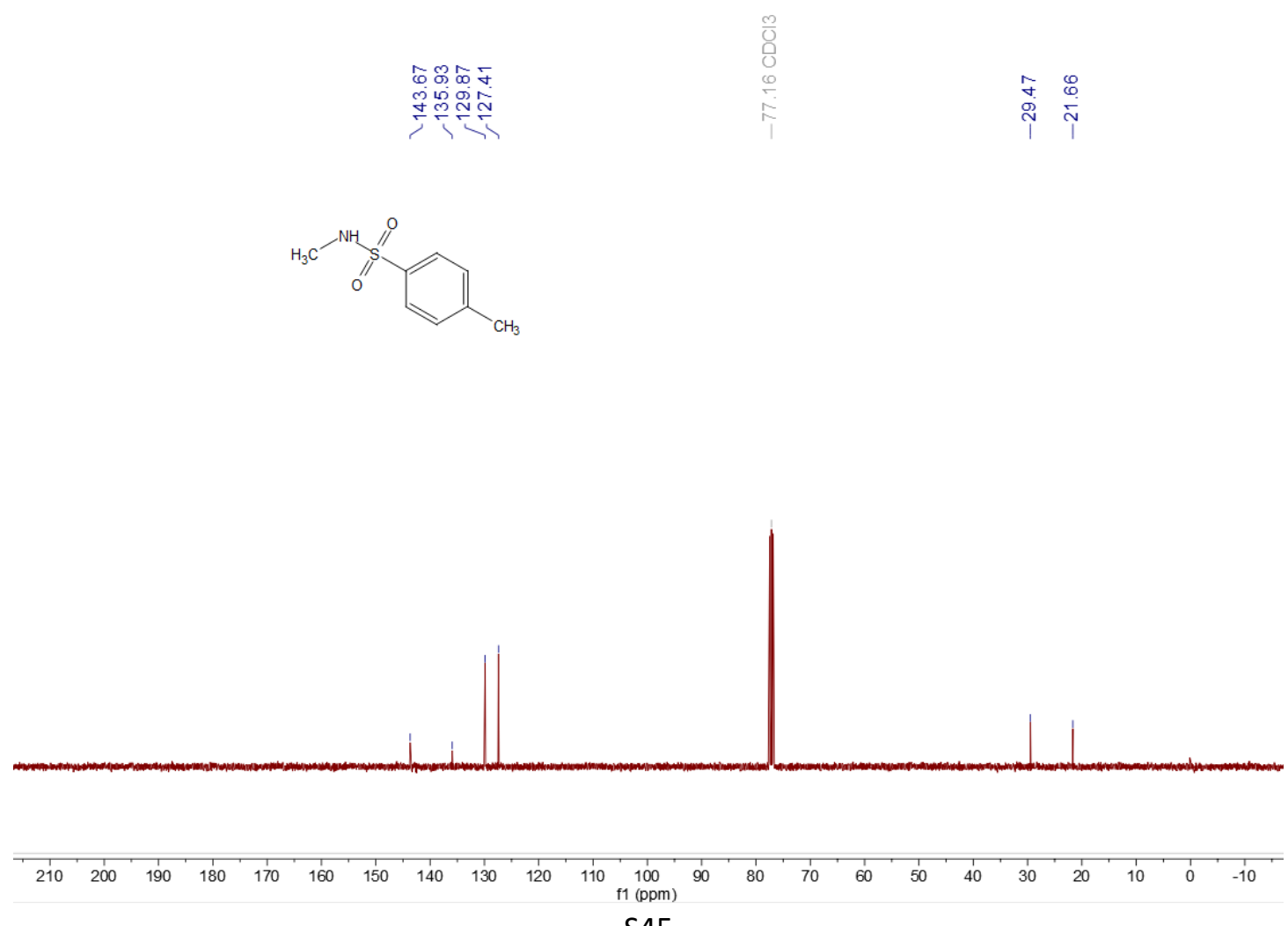

S45 


\section{Compound 3ga}
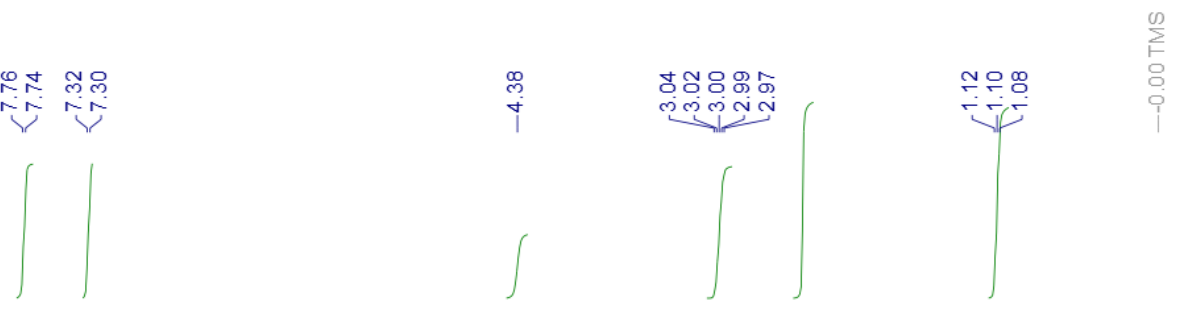<smiles>CCNS(=O)(=O)c1ccc(C)cc1</smiles>

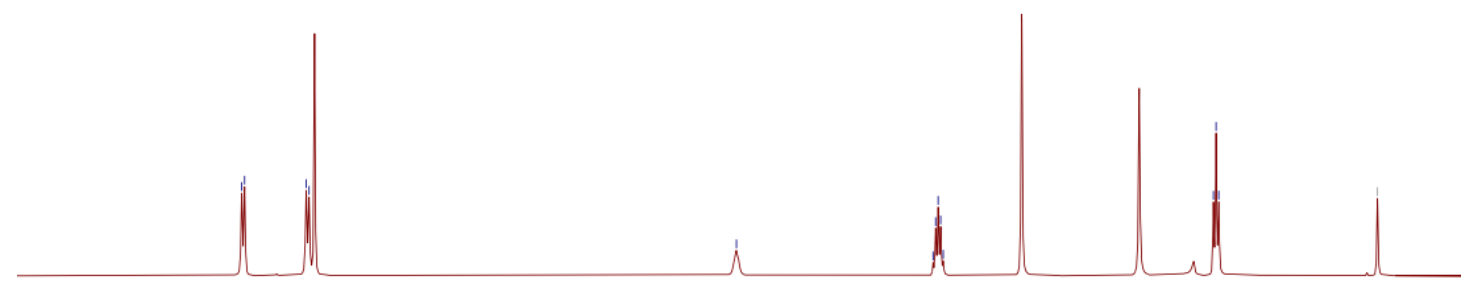

\begin{tabular}{|c|c|c|c|c|c|c|c|c|c|c|c|c|c|c|c|c|c|c|}
\hline & & & & & & & & & 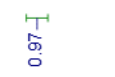 & & 'T & 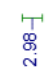 & & & 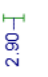 & & & \\
\hline 9.0 & 8.5 & 8.0 & 7.5 & 7.0 & 6.5 & 6.0 & 5.5 & 5.0 & $\begin{array}{l}4.5 \\
\text { f1 (ppm) }\end{array}$ & 3.5 & 3.0 & 2.5 & 20 & 1.5 & 1.0 & 0.5 & 0.0 & -0.5 \\
\hline
\end{tabular}

ณำ

뜸ํㅡㄴ

$5 \div$

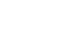

,<smiles>CCNS(=O)(=O)c1ccc(C)cc1</smiles>

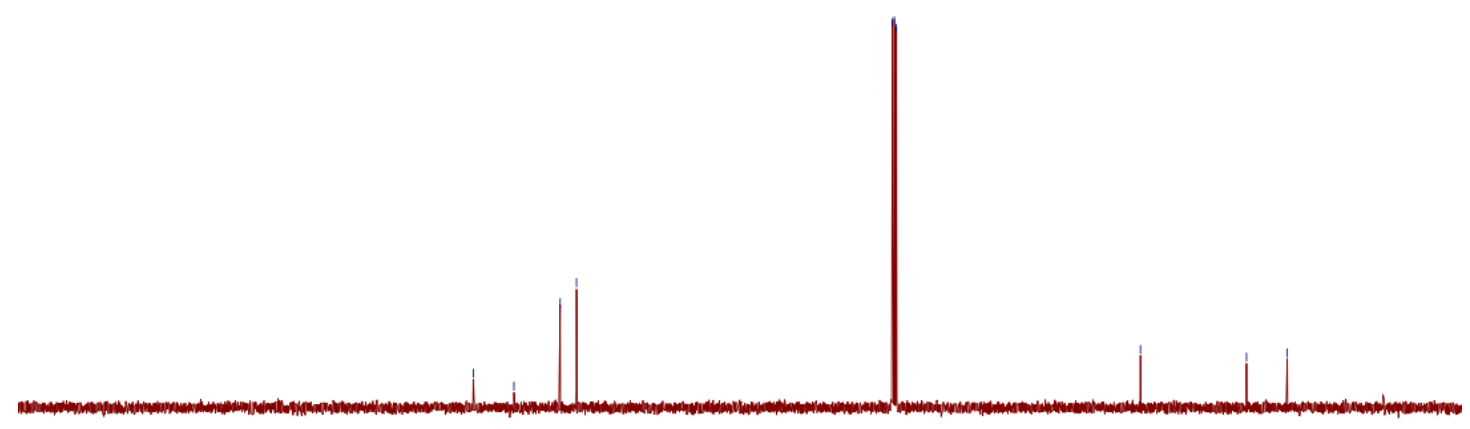




\section{Compound 3ha}
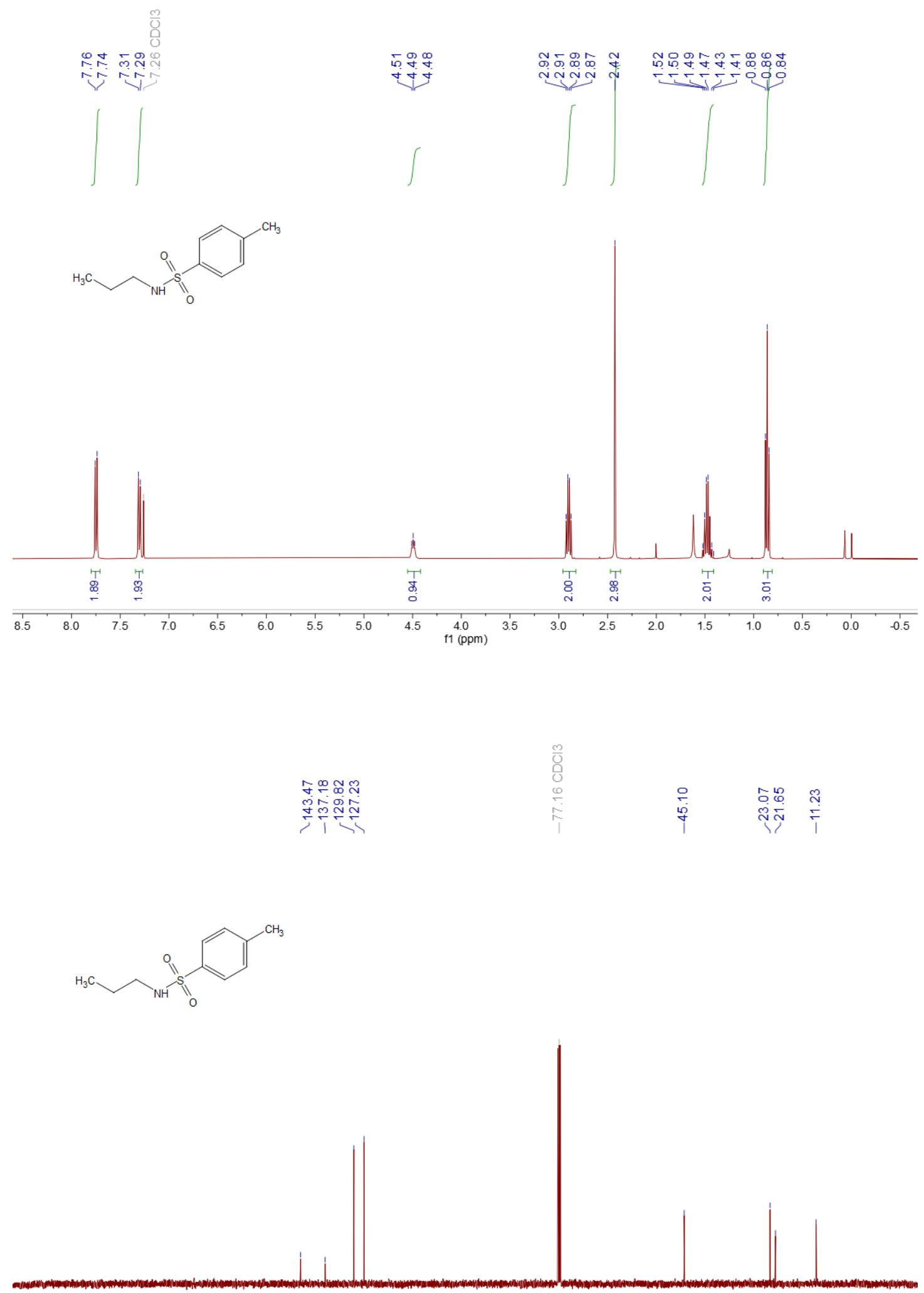

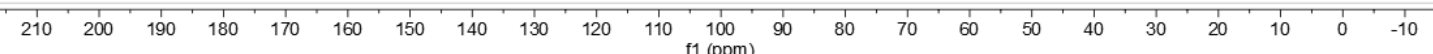




\section{Compound 3ia}

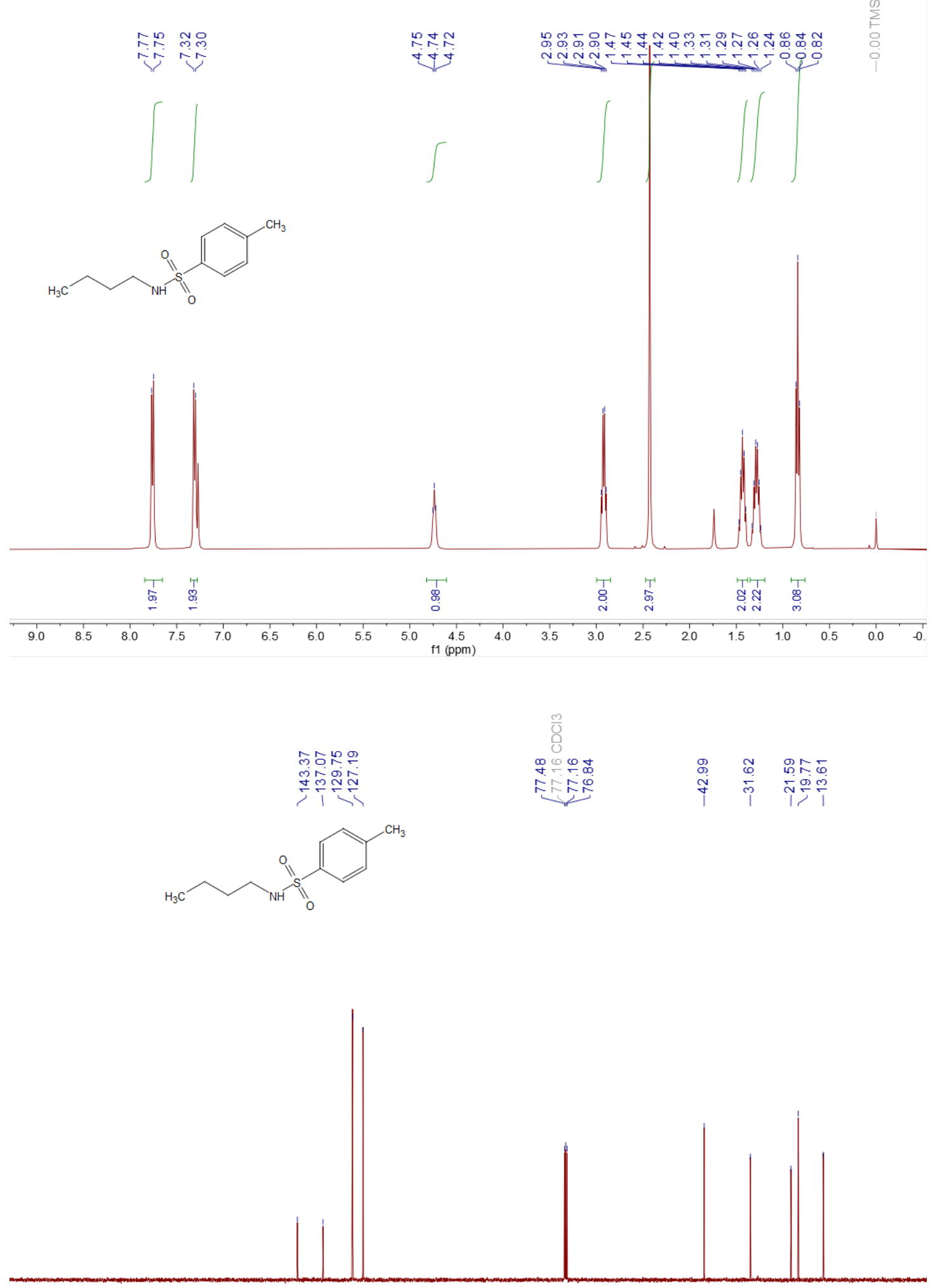

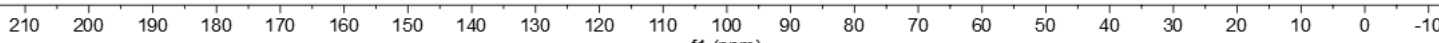




\section{Compound 3ja}
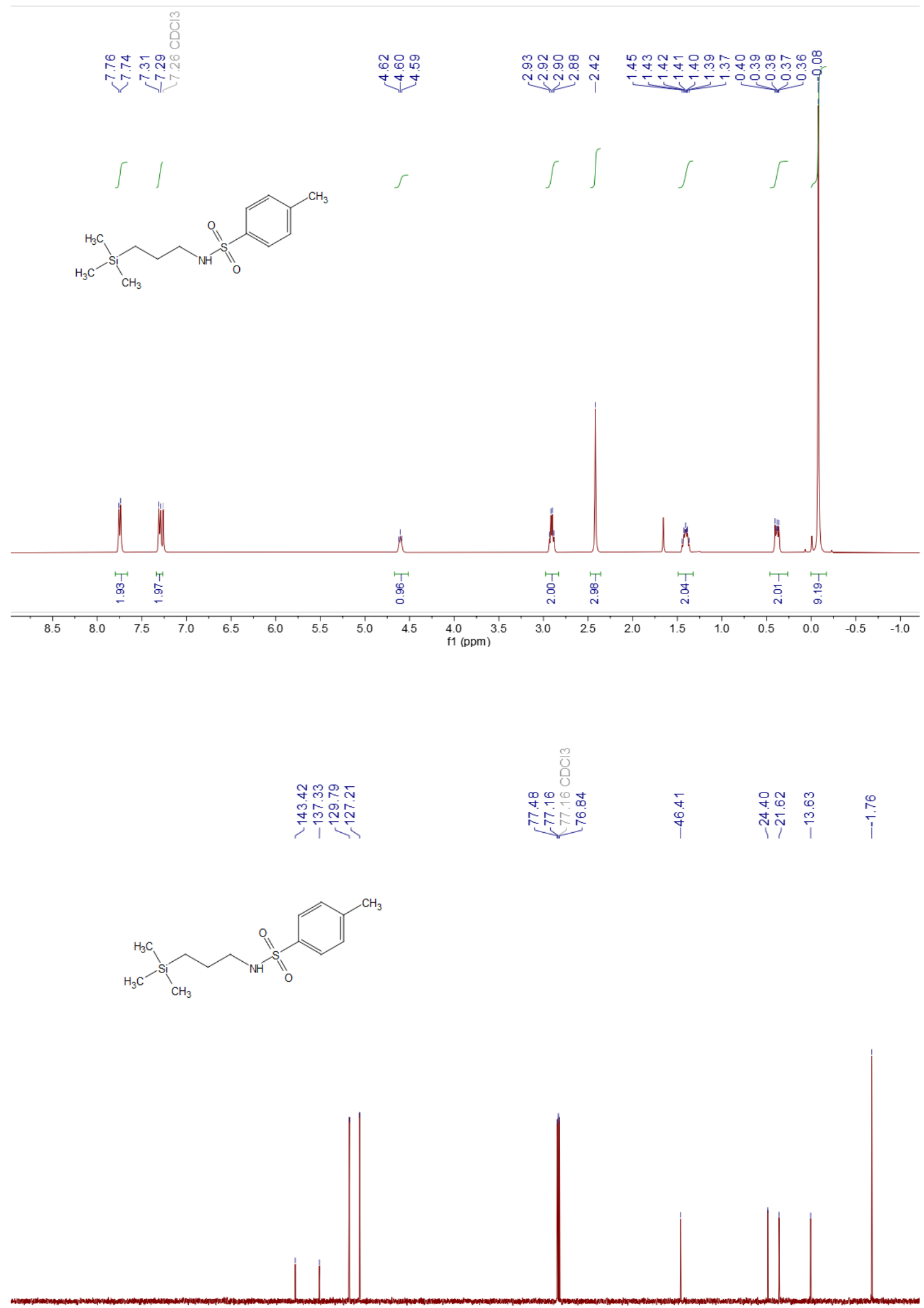

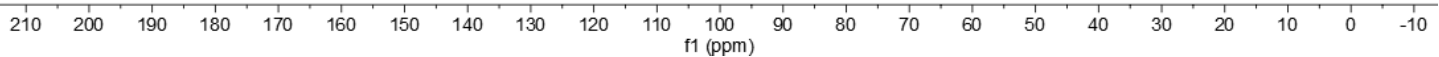




\section{Compound 3ka}

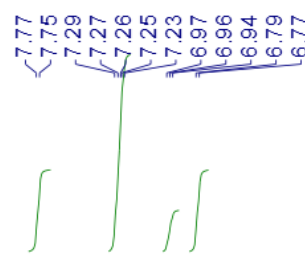

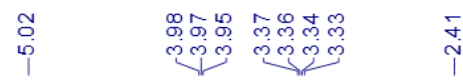<smiles>Cc1ccc(S(=O)(=O)NCCOc2ccccc2)cc1</smiles>
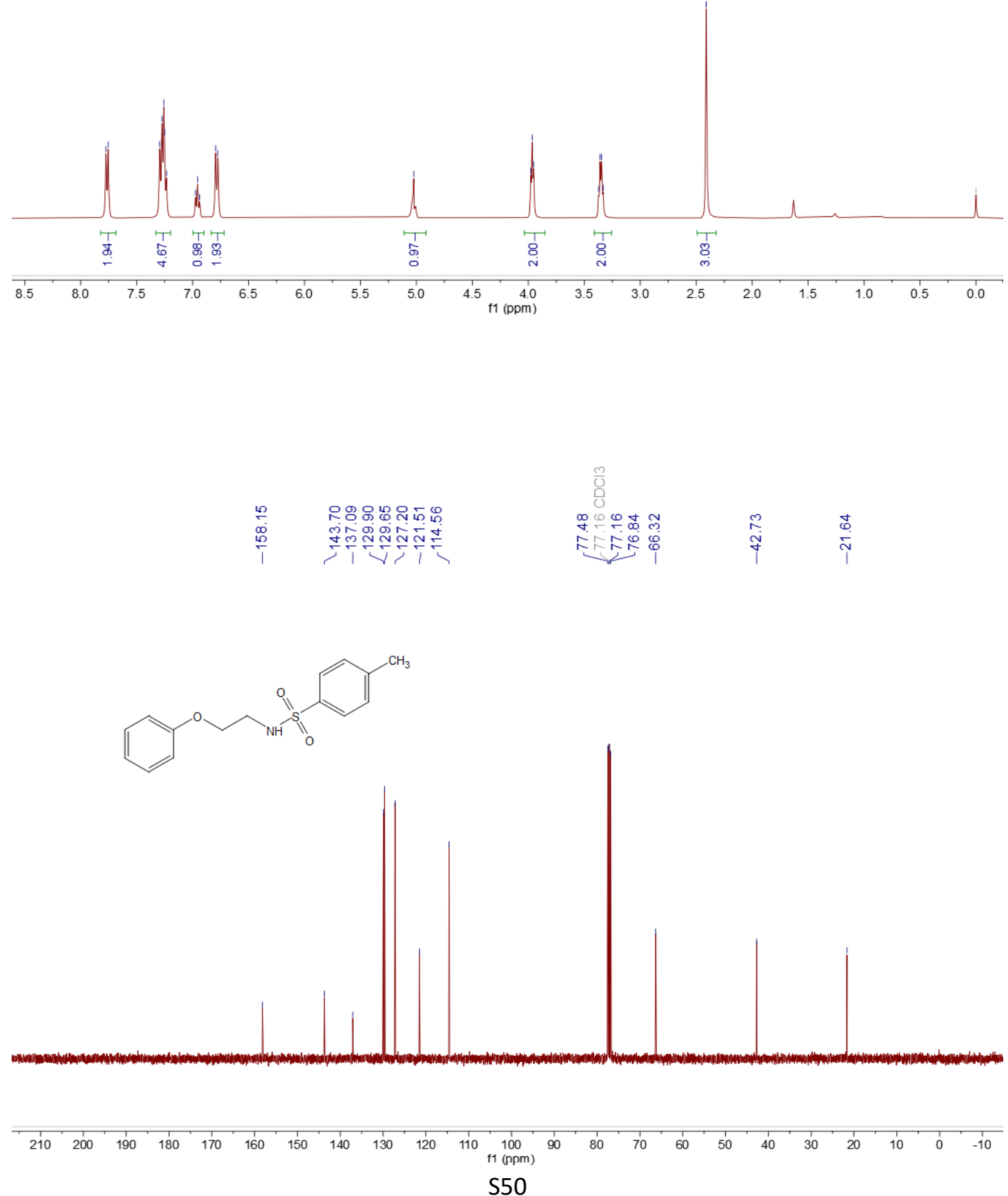
Compound 3la
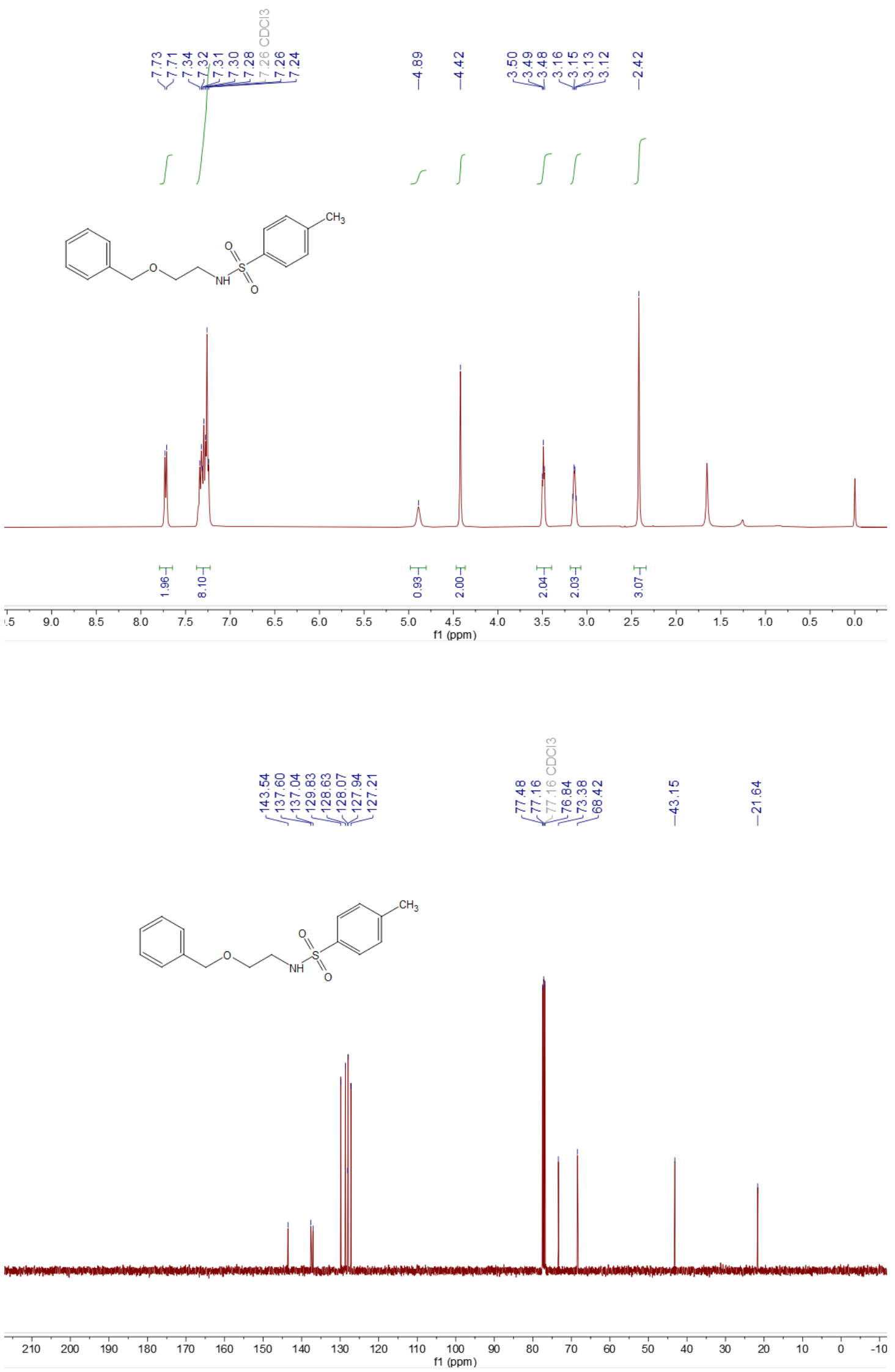

S51 


\section{Compound 3ma}
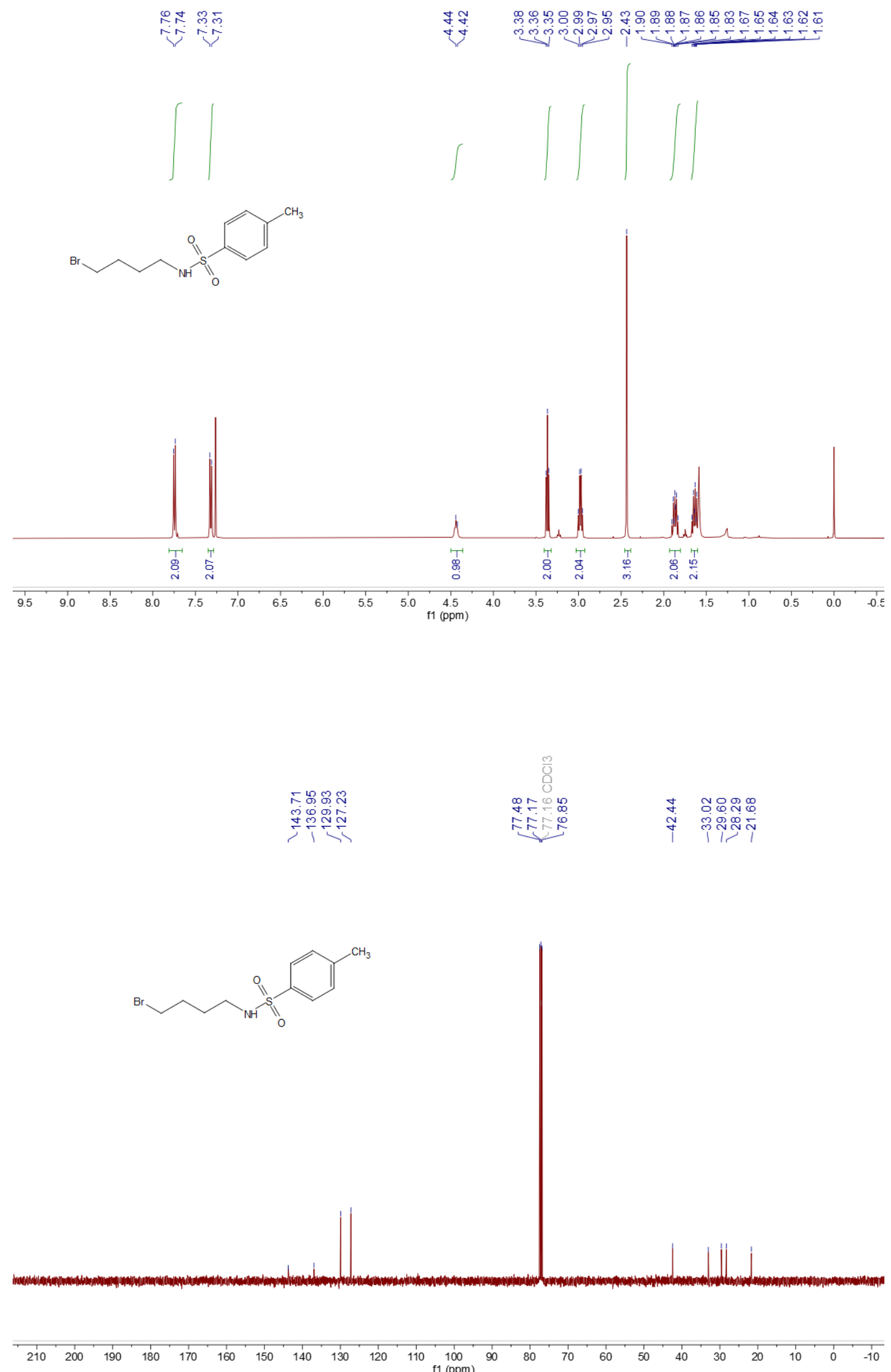


\section{Compound 3na}
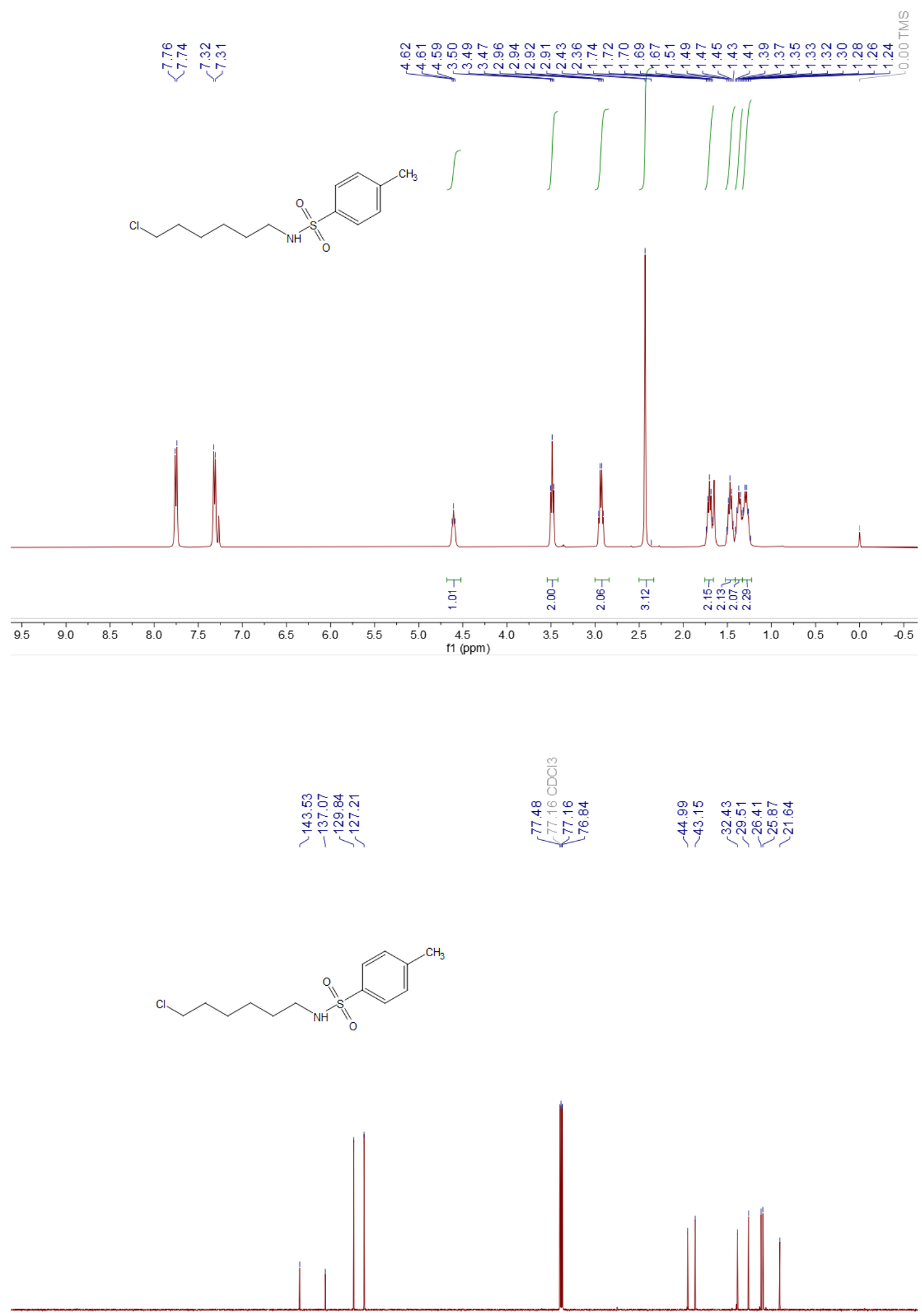

$\begin{array}{llllllllllll}210 & 200 & 190 & 180 & 170 & 160 & 150 & 140 & 130 & 120 & 110 & \begin{array}{c}100 \\ \mathrm{f} 1(\mathrm{ppm})\end{array}\end{array}$ 


\section{Compound 3oa}

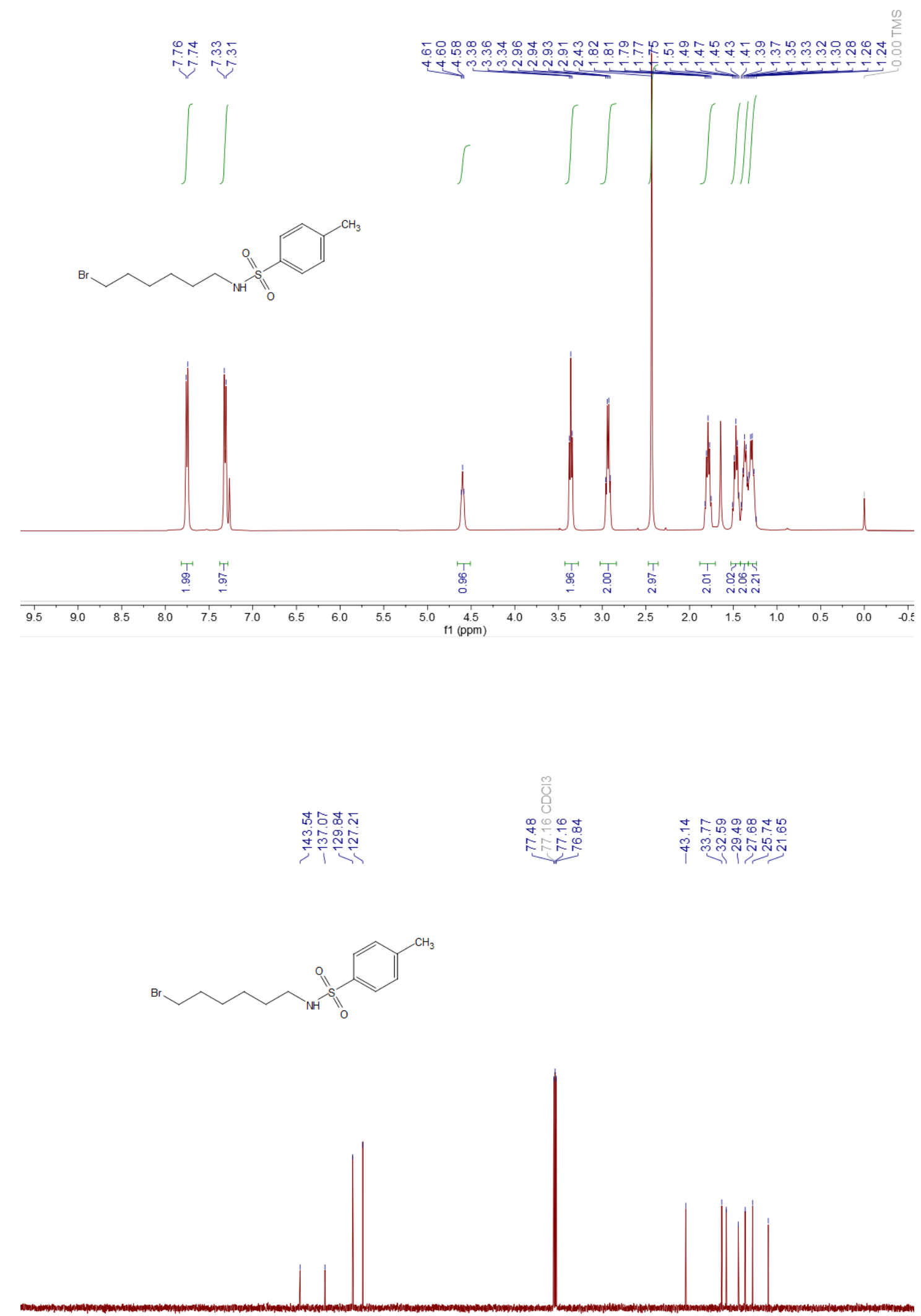

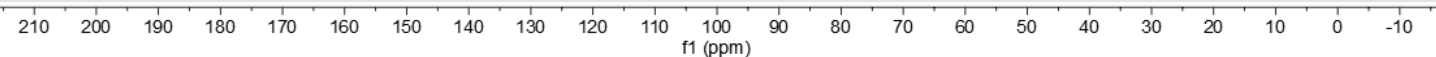




\section{Compound 3pa}
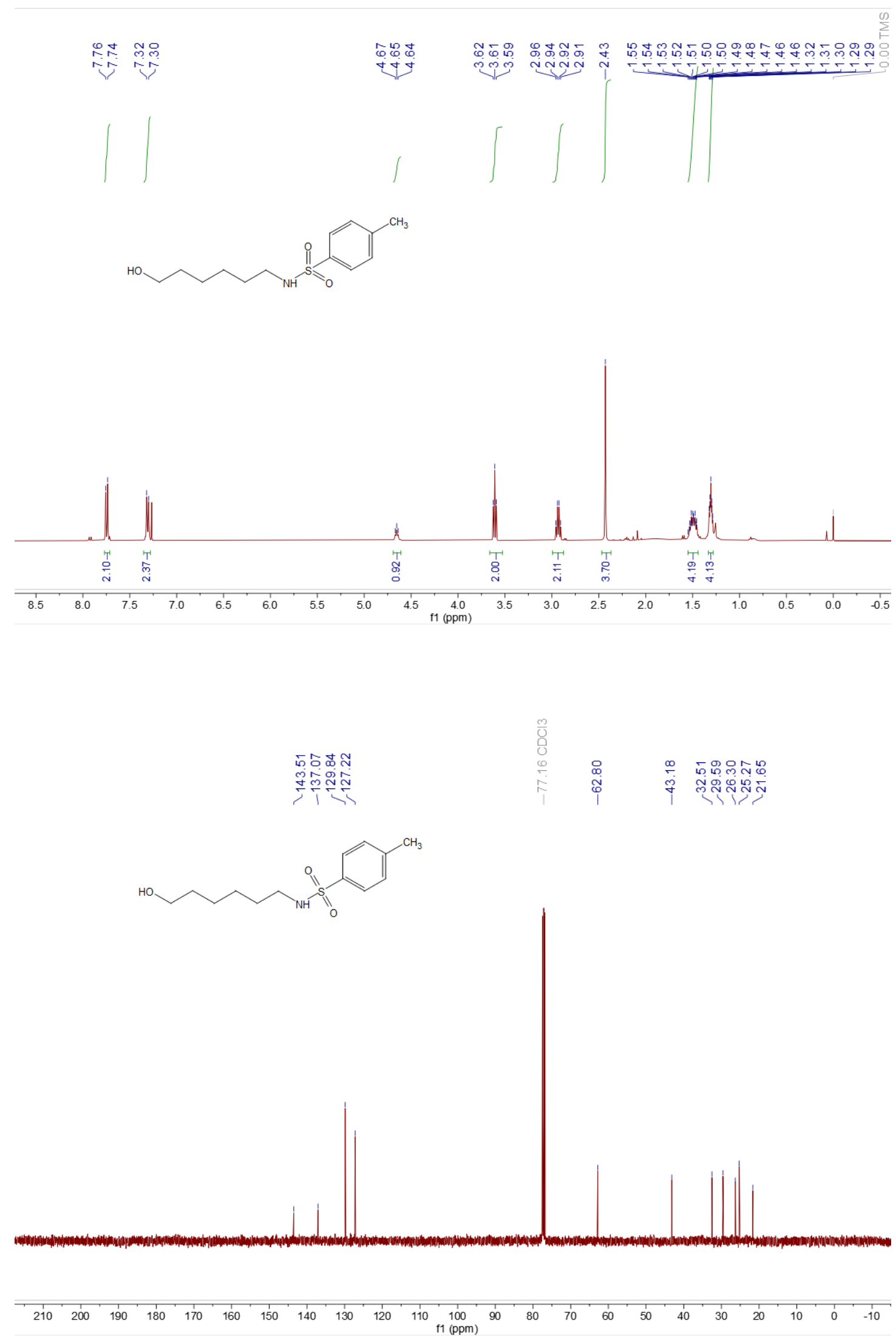


\section{Compound 3qa}
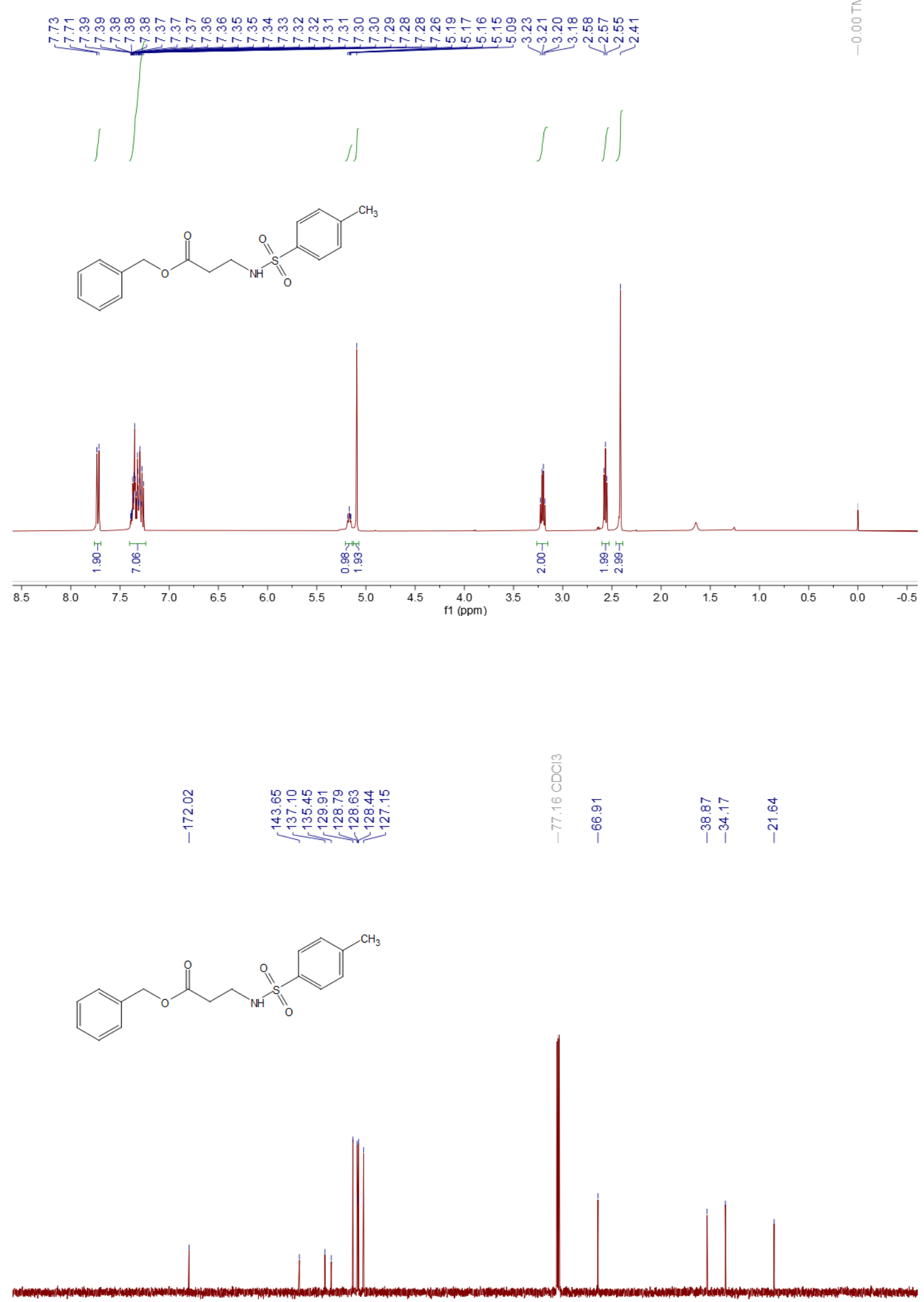

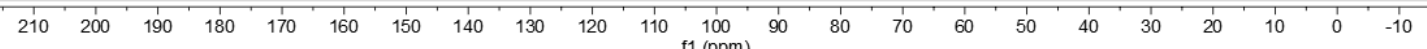




\section{Compound 3ra}

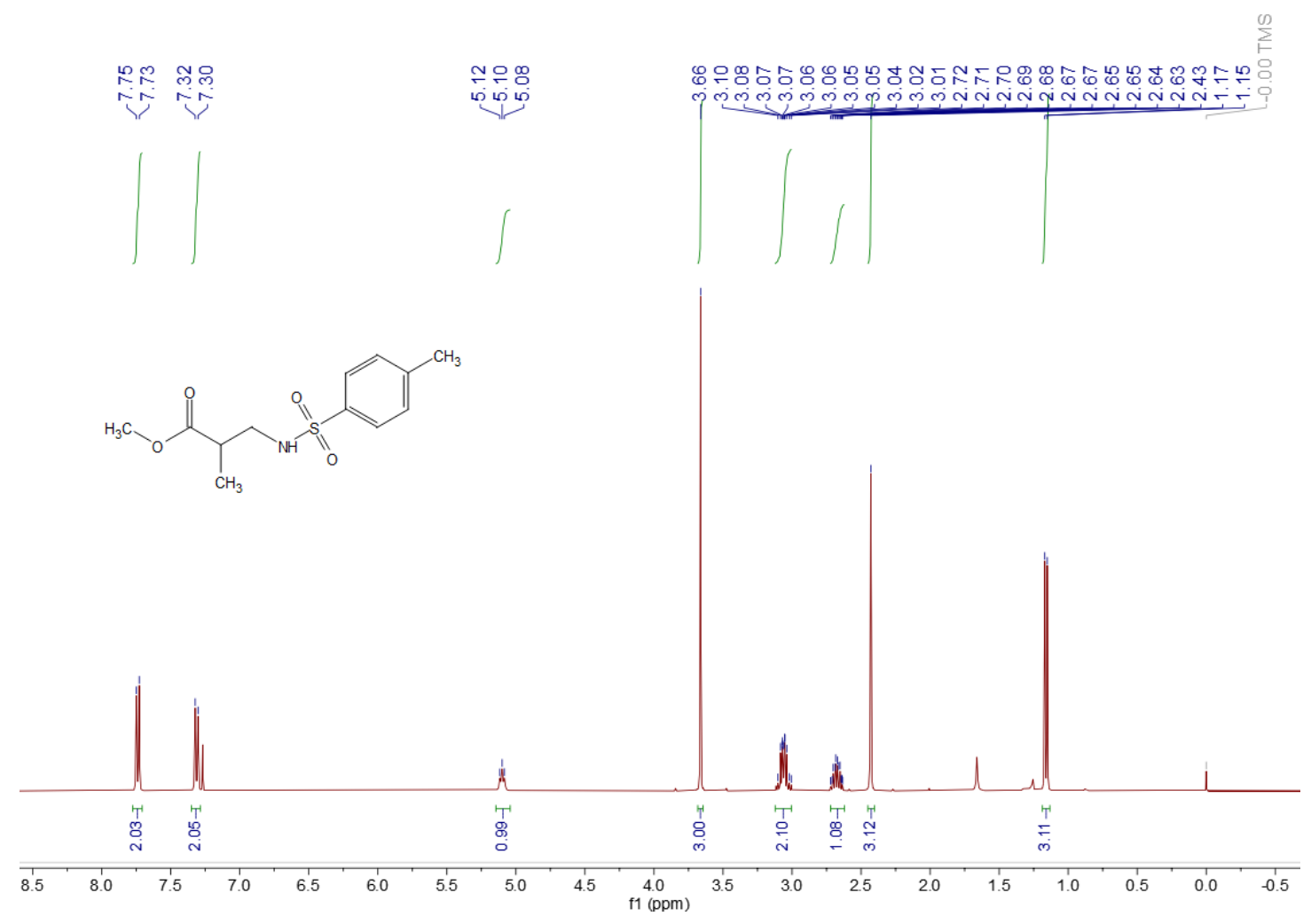

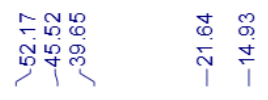<smiles>COC(=O)C(C)CNS(=O)(=O)c1ccc(C)cc1</smiles>

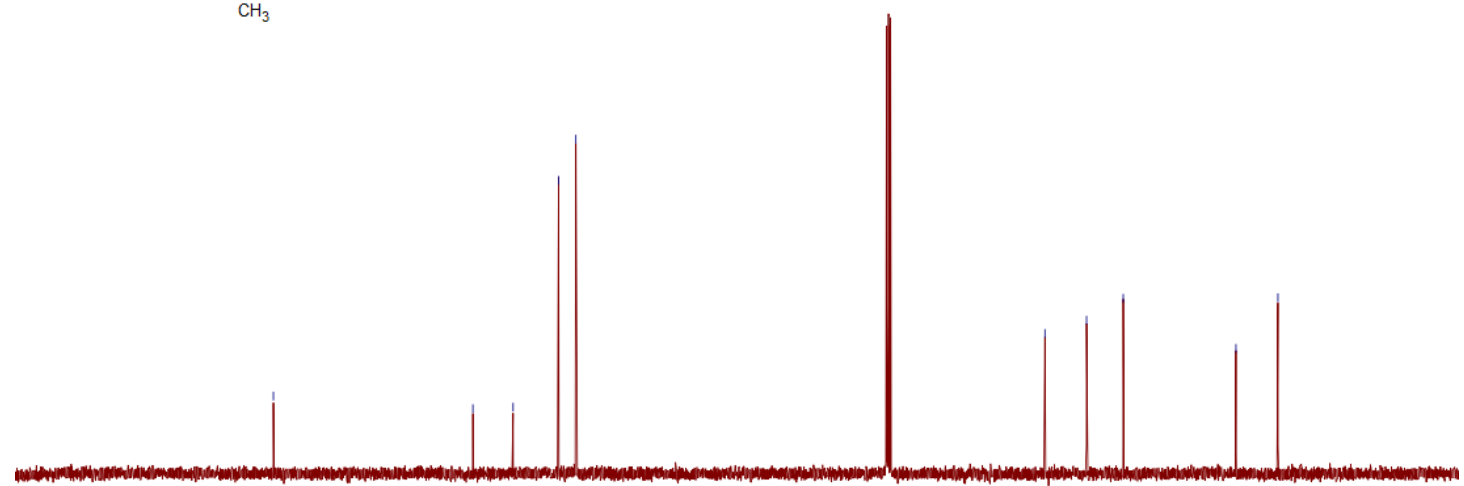

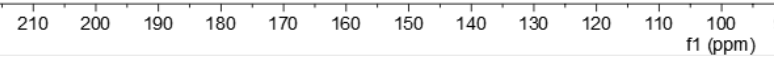




\section{Compound 3sa}

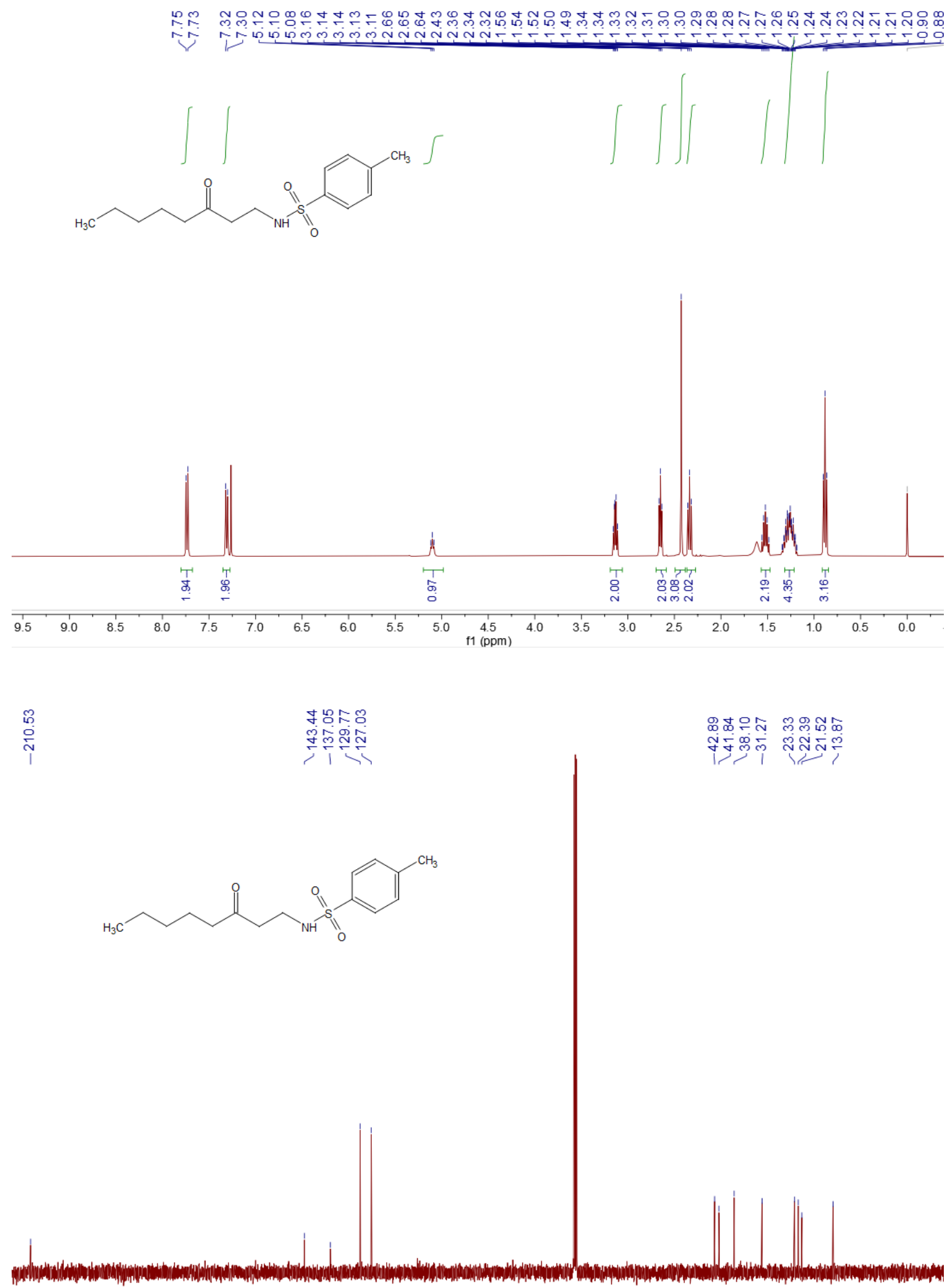

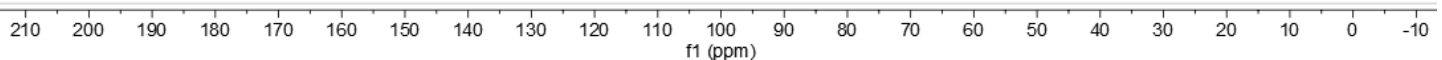




\section{Compound 3ta}
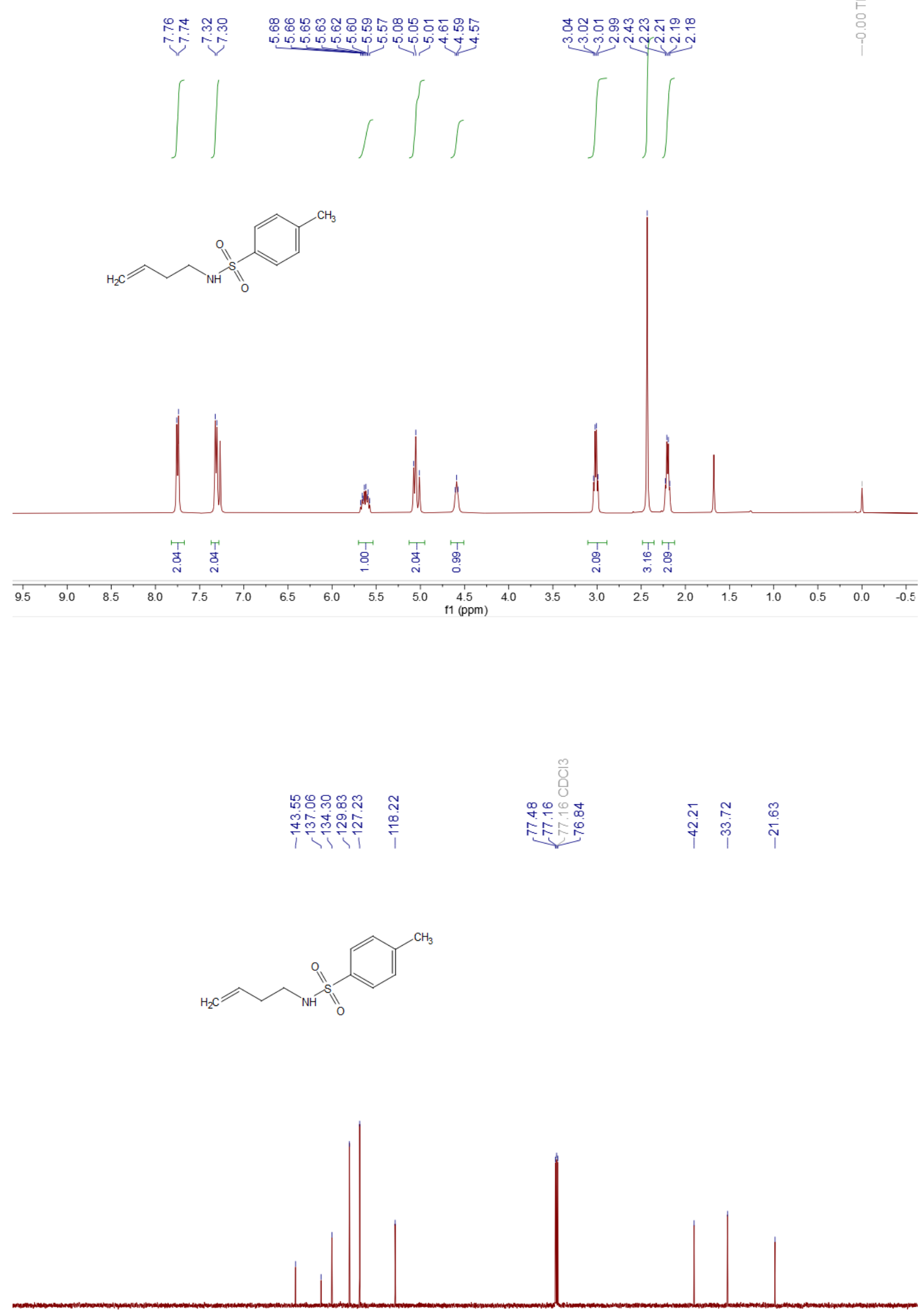

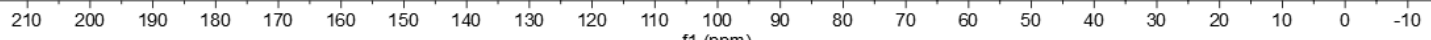




\section{Compound 3ua}

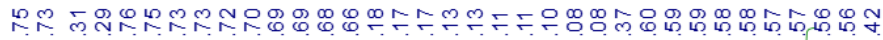

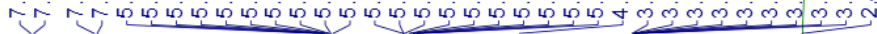

$\mid$<smiles>C=CCNS(=O)(=O)c1ccc(C)cc1</smiles>

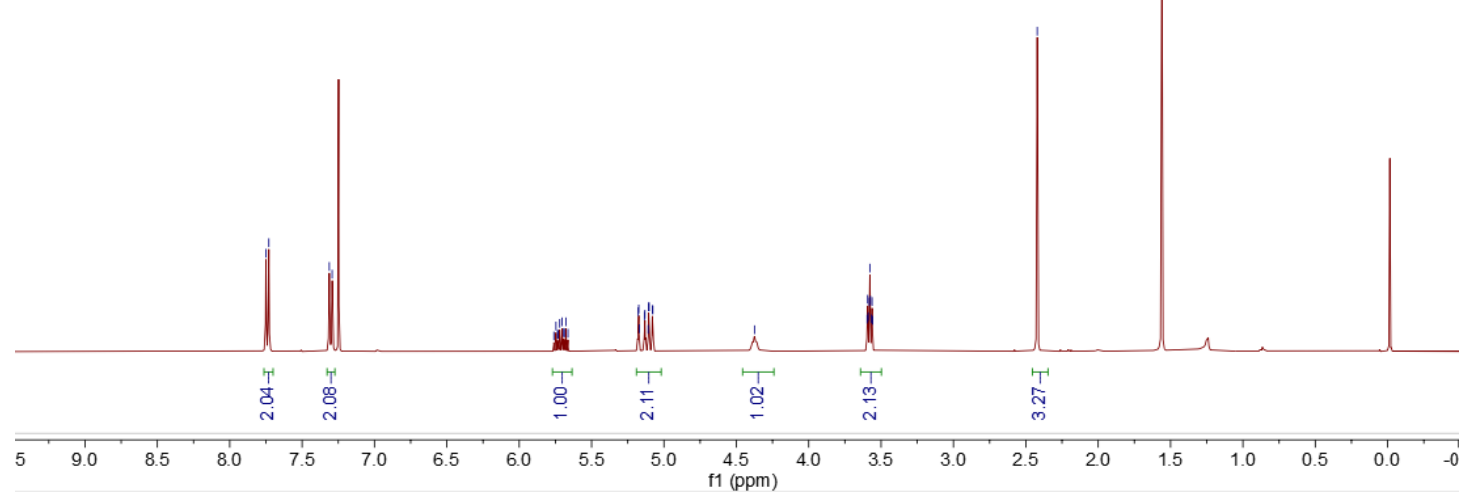

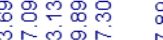

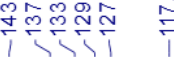

制
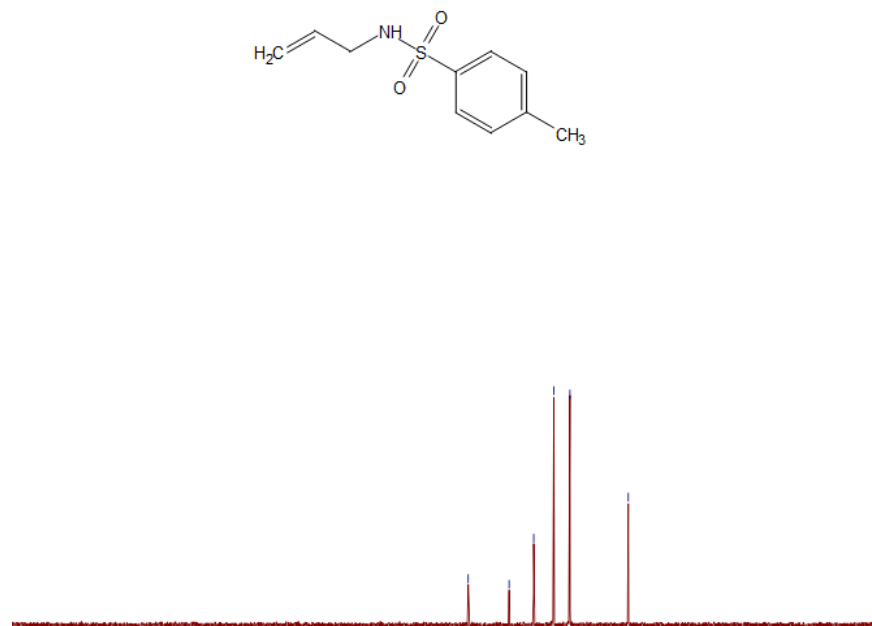


\section{Compound 3za}

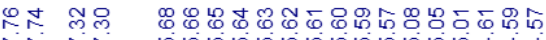

访

minjinañ
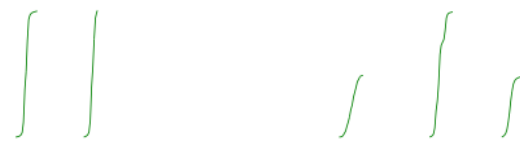<smiles>C=CCCNS(=O)(=O)c1ccc(C)cc1</smiles>

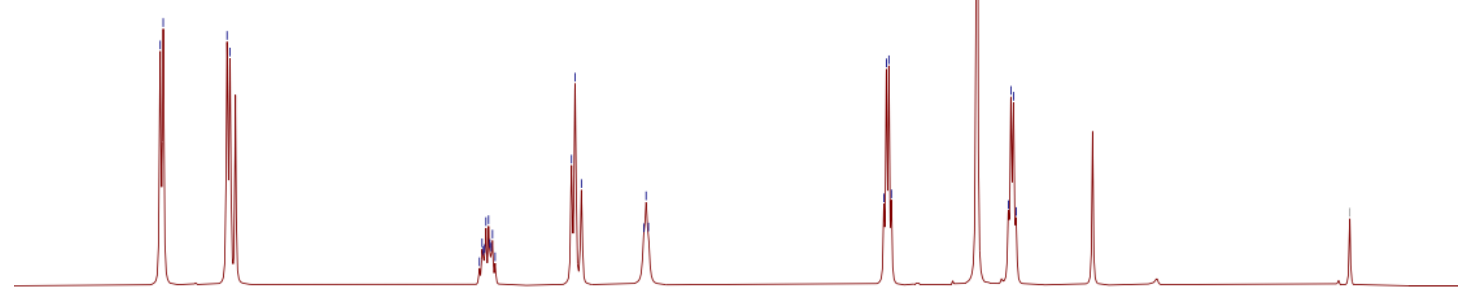

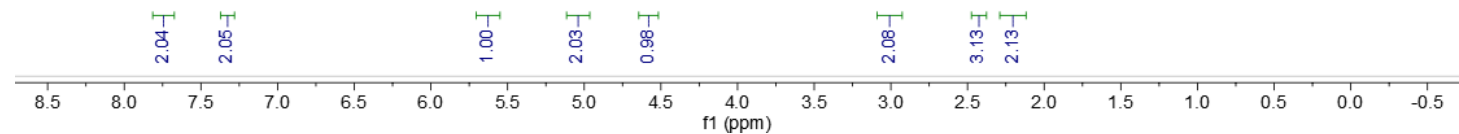

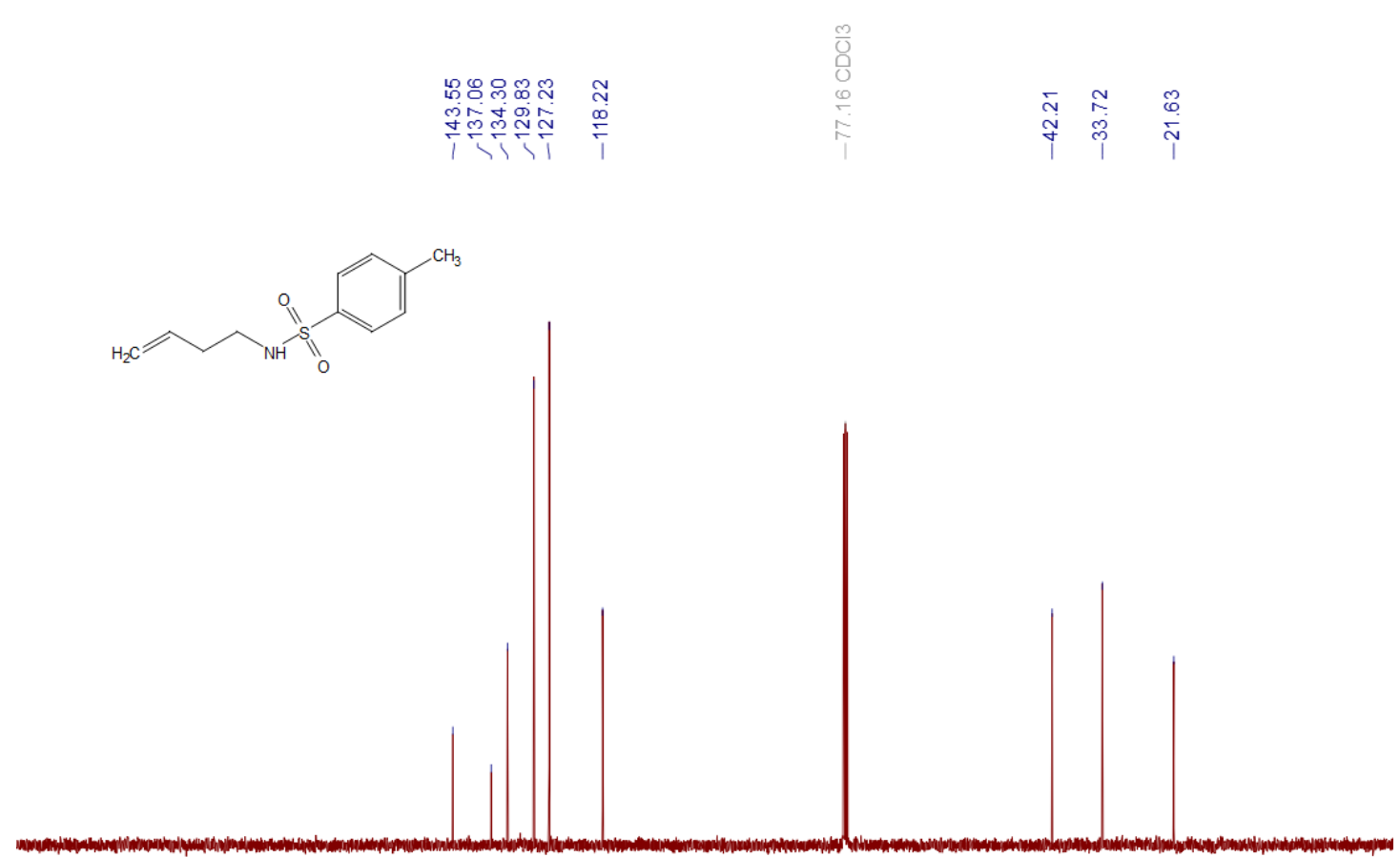

$\begin{array}{llllllllll}210 & 200 & 190 & 180 & 170 & 160 & 150 & 140 & 130 & 120\end{array}$ $110 \begin{gathered}100 \\ 11(\mathrm{ppm})\end{gathered}$ 


\section{Compound 5aa}
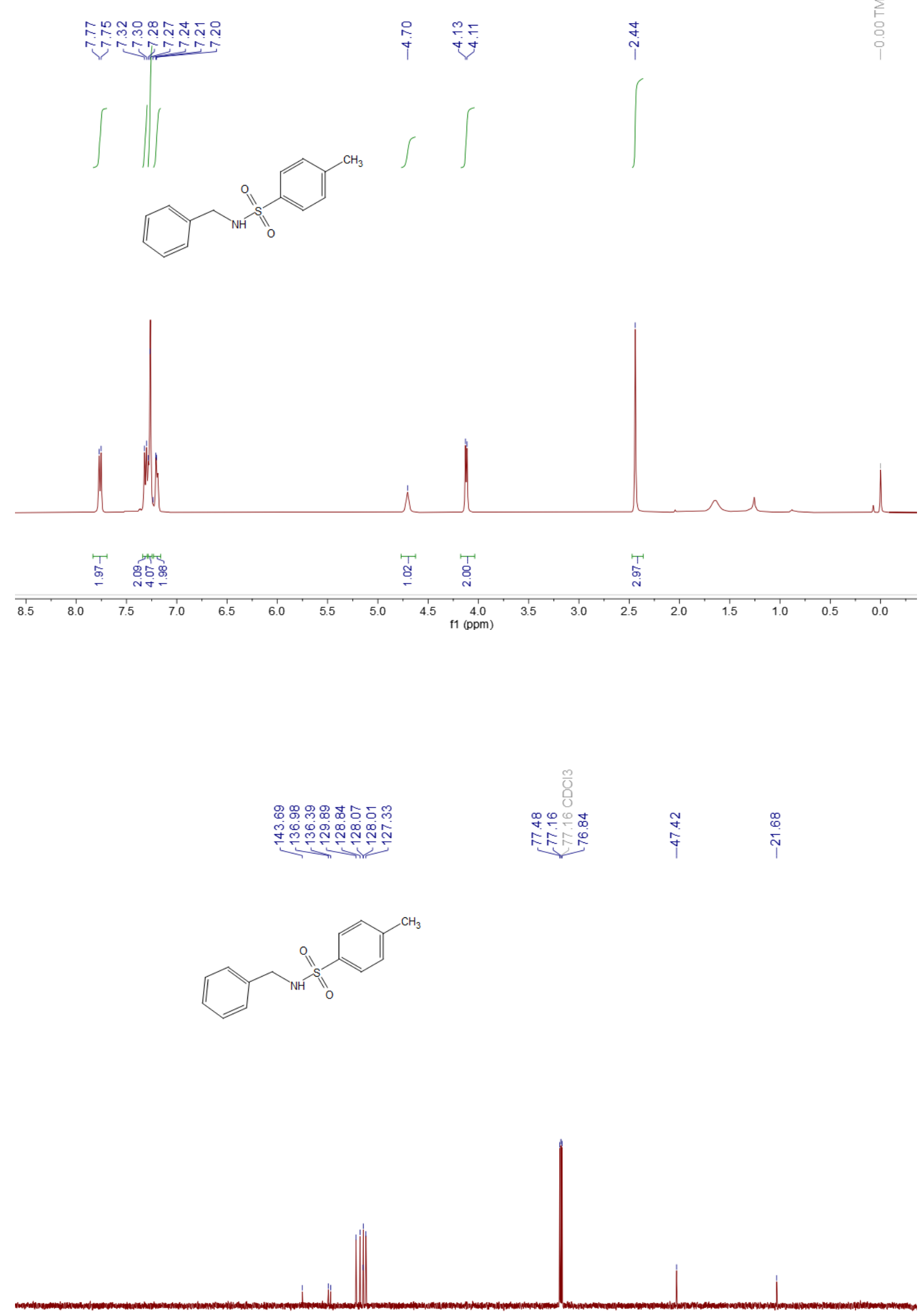

$\begin{array}{lllllllllllllllllllllllllllllllllll} & 210 & 200 & 190 & 180 & 170 & 160 & 150 & 140 & 130 & 120 & 110 & 100 & 90 & 80 & 70 & 60 & 50 & 40 & 30 & 20 & 10 & 0 & -10\end{array}$ 


\section{Compound 5ba}

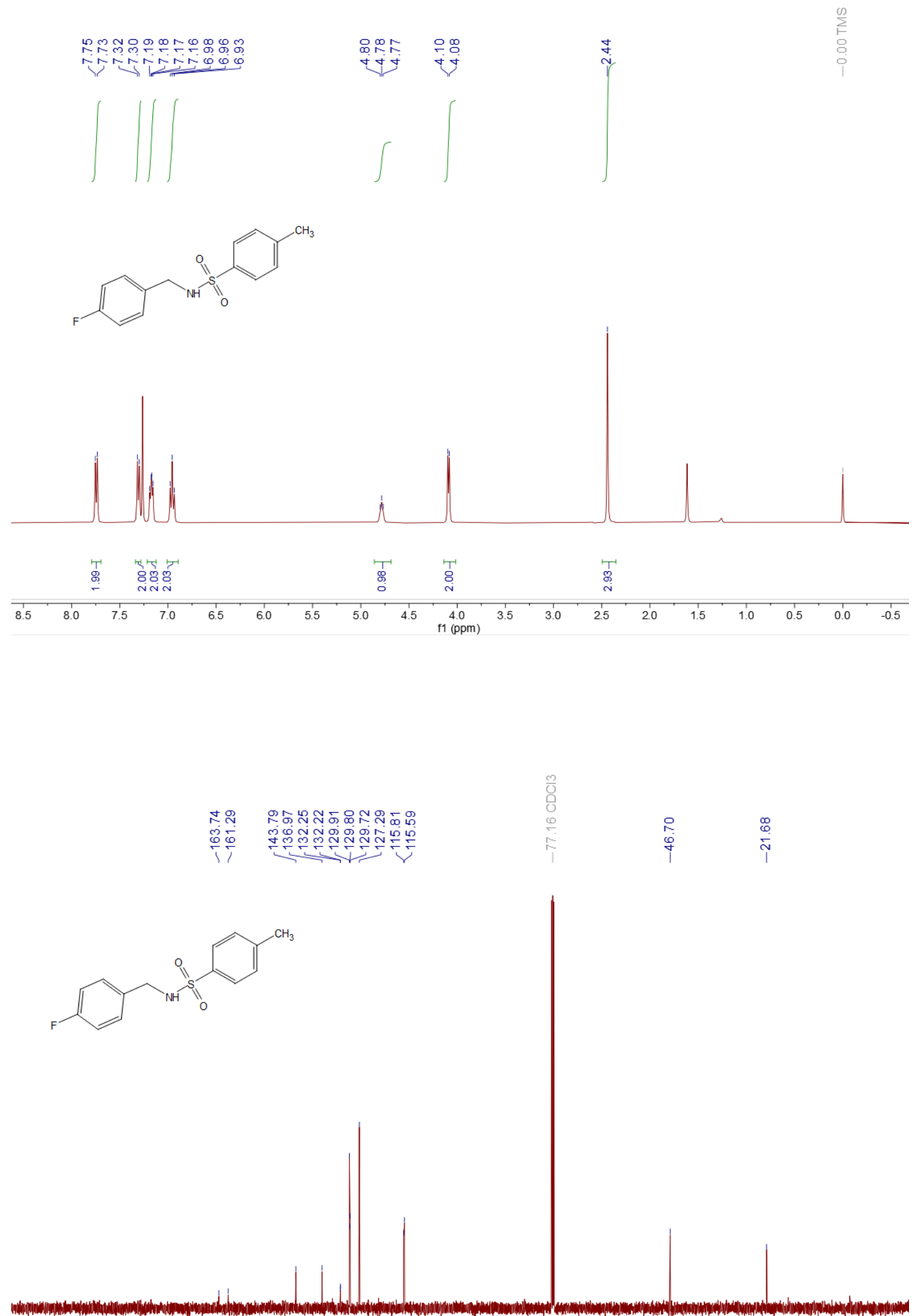

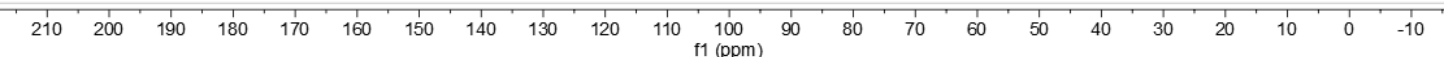




\section{Compound 5ca}
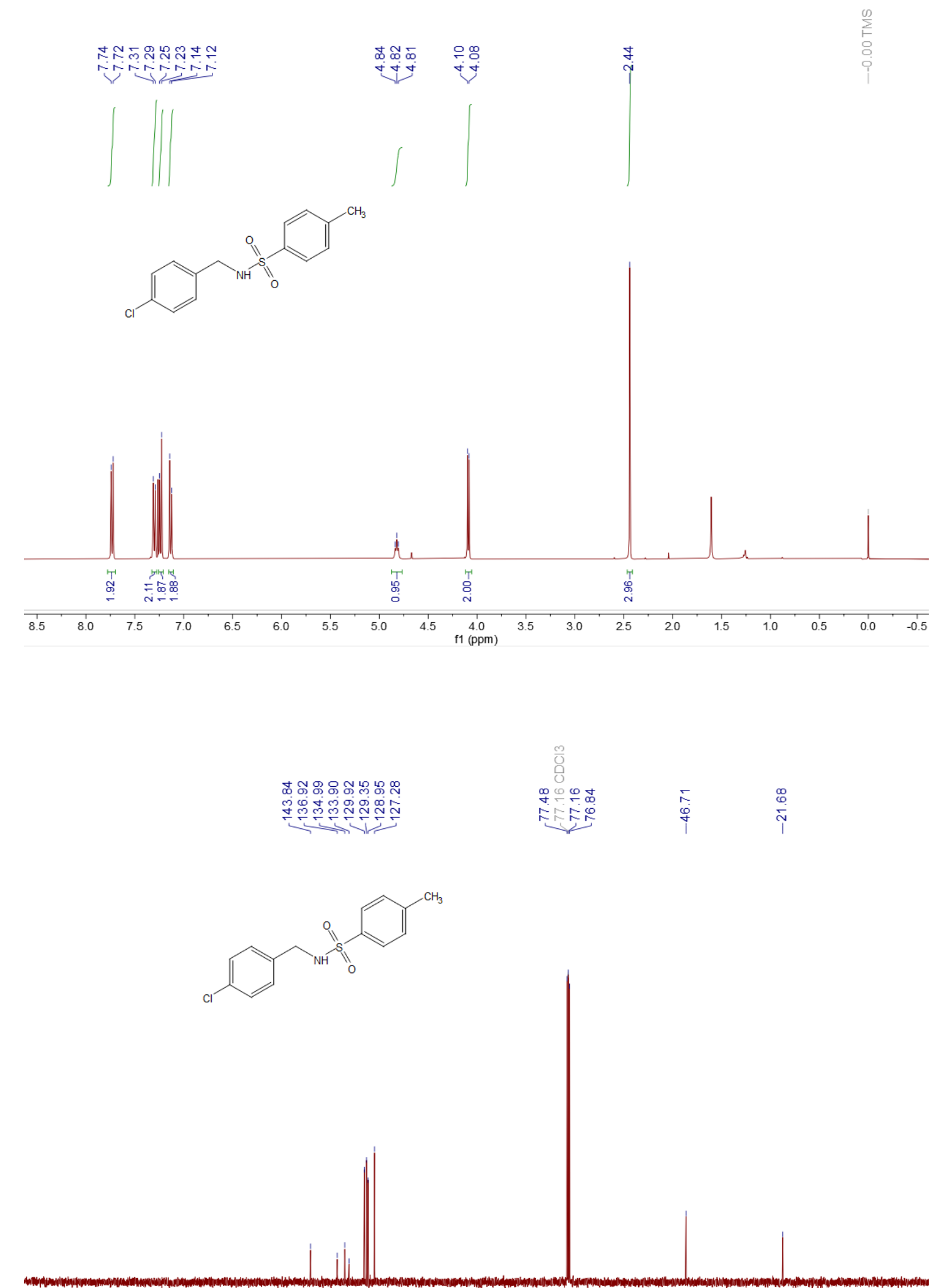

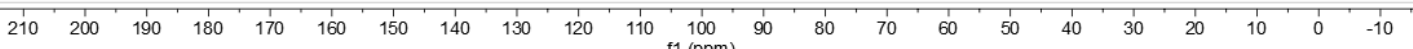




\section{Compound 5da}

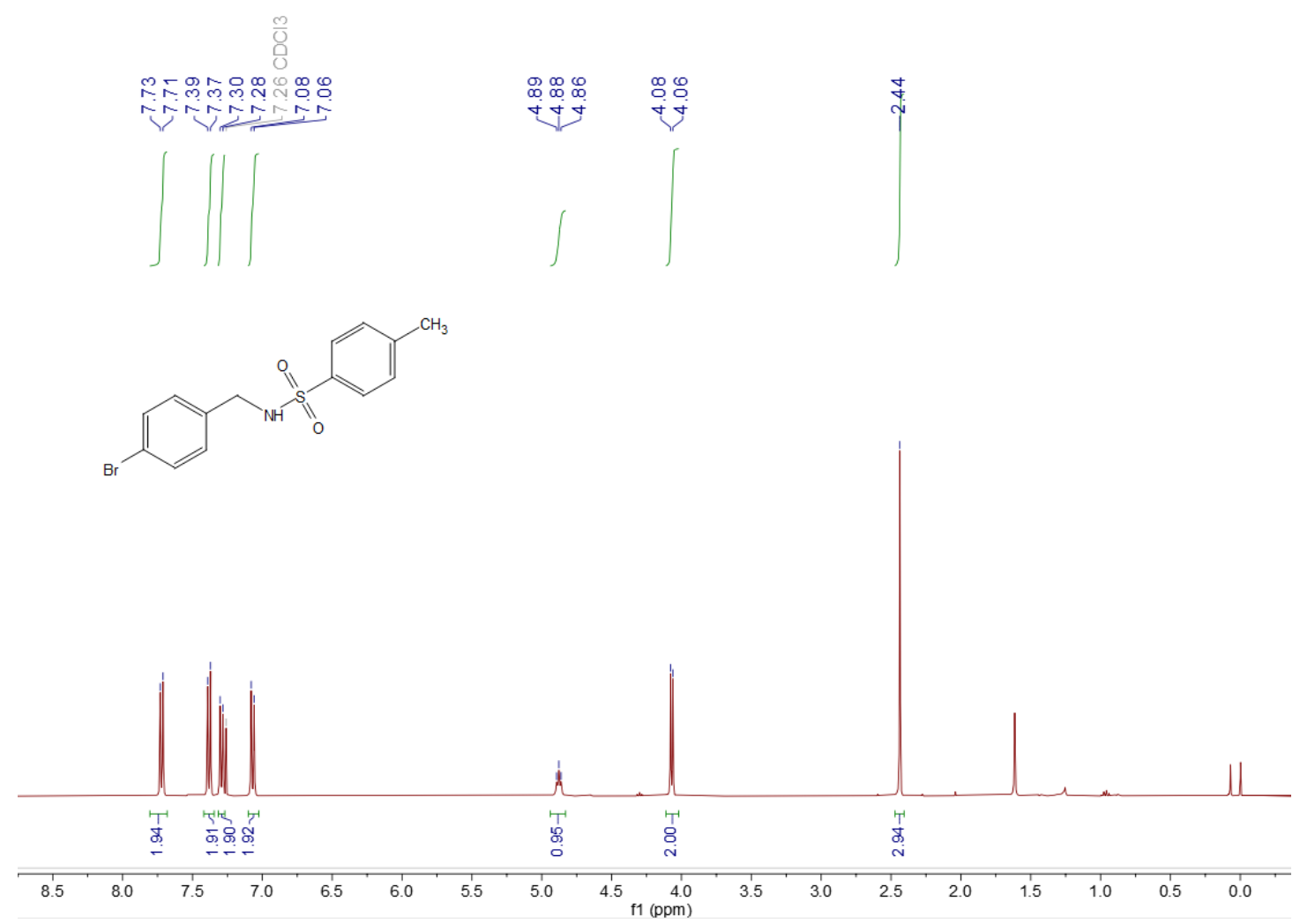

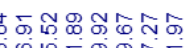

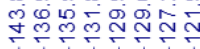

$\stackrel{\infty}{\infty} \stackrel{\infty}{\sim}$

№

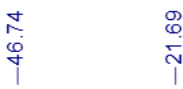
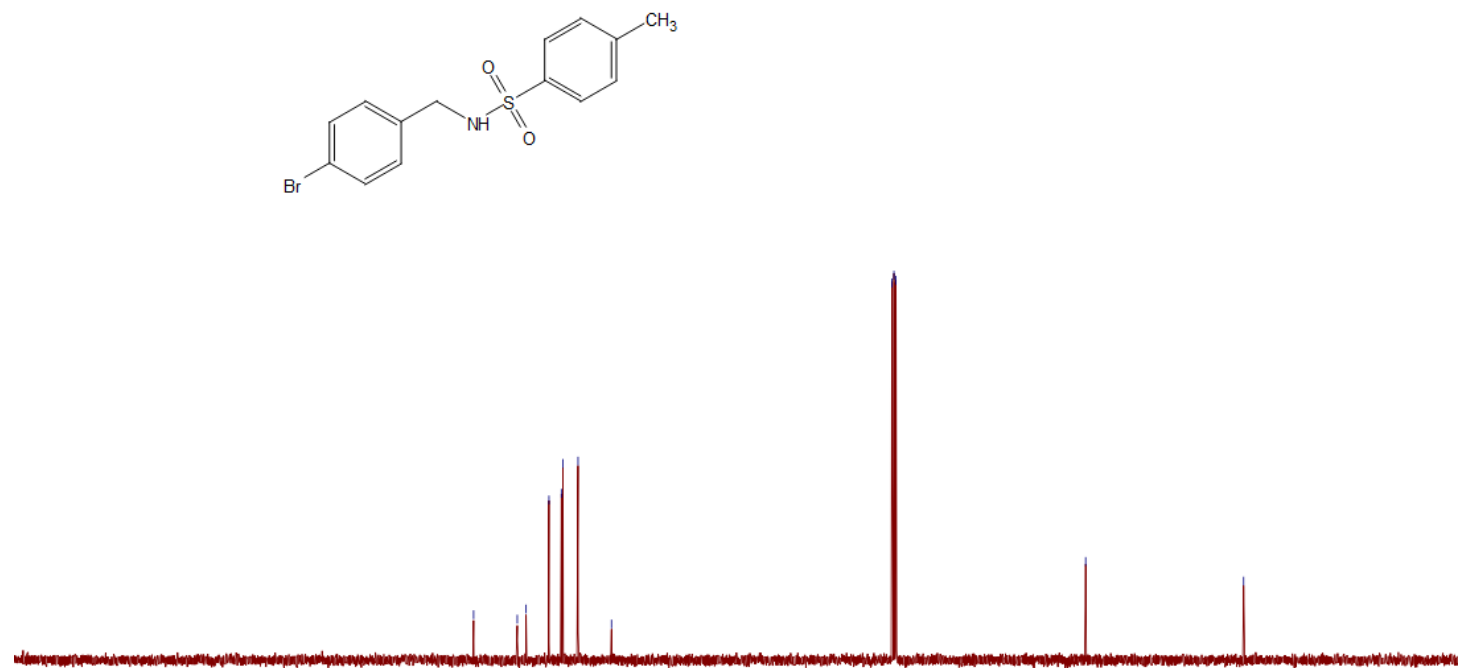

210

170

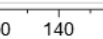

1301

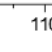

100 


\section{Compound 5ea}
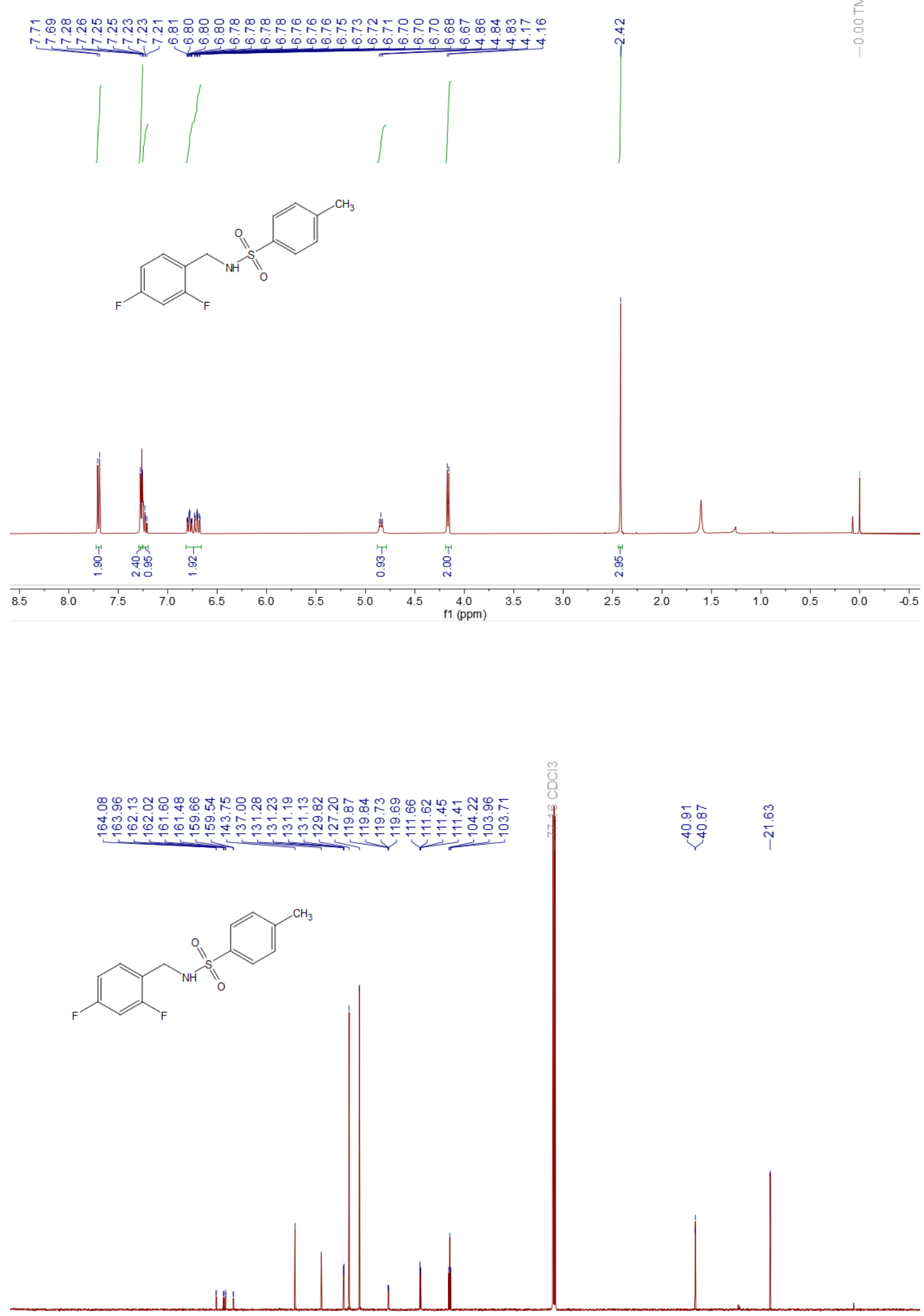

$\begin{array}{lllllllllllll}210 & 200 & 190 & 180 & 170 & 160 & 150 & 140 & 130 & 120 & 110 & 100 & 90\end{array}$ 


\section{Compound 5fa}
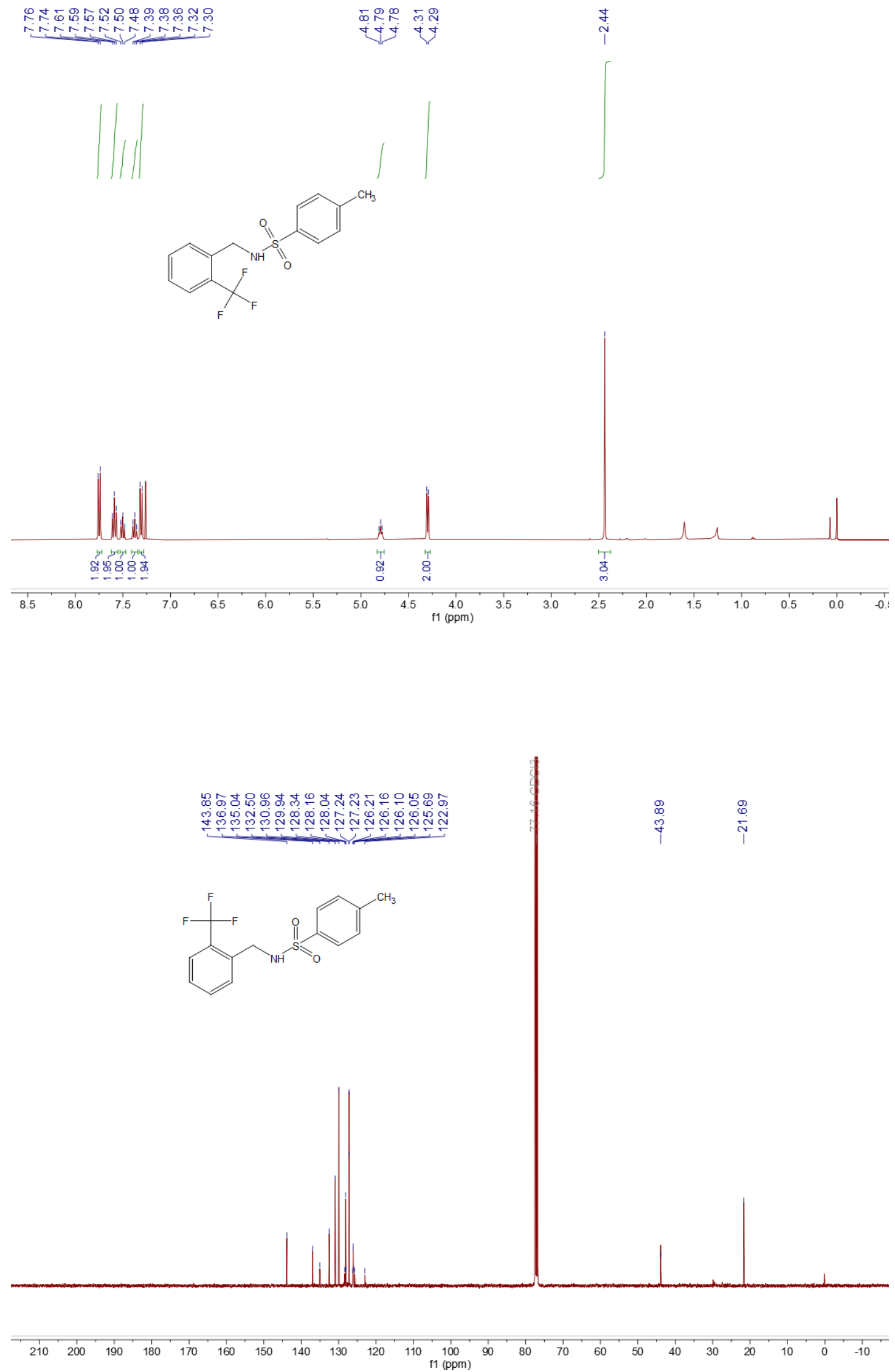


\section{Compound 5ga}
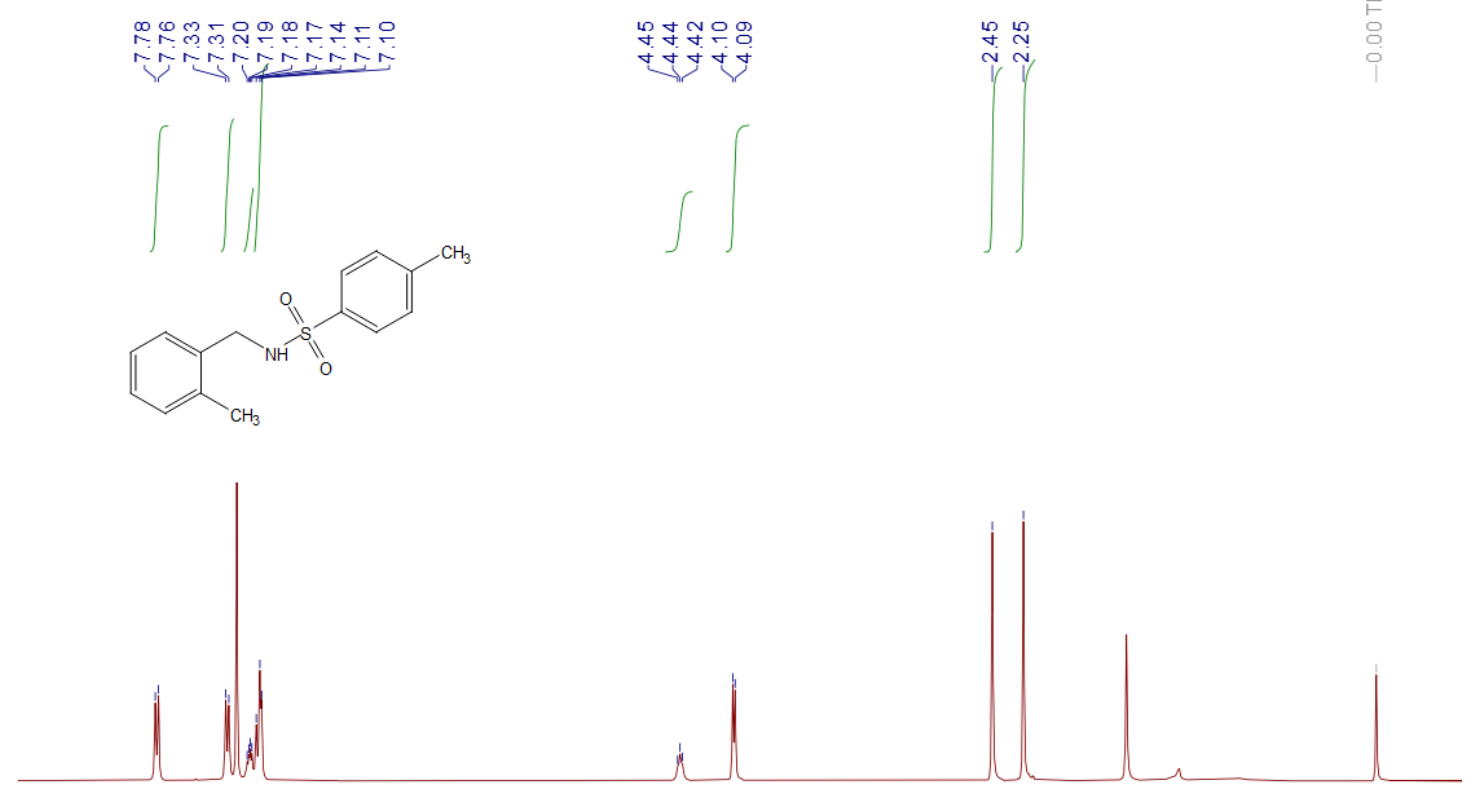

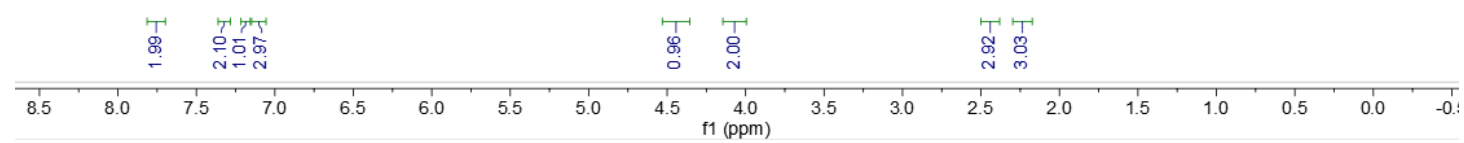
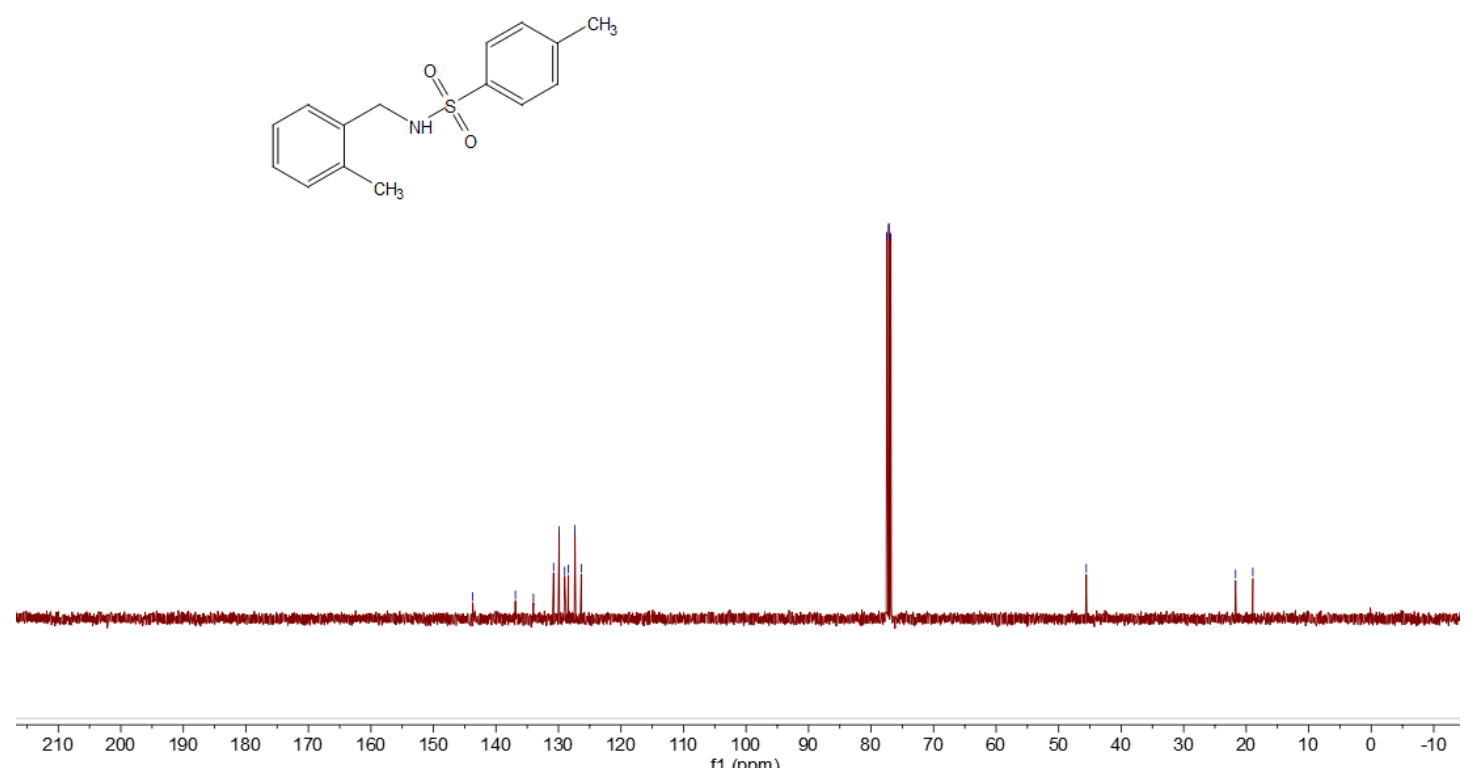


\section{Compound 5ha}

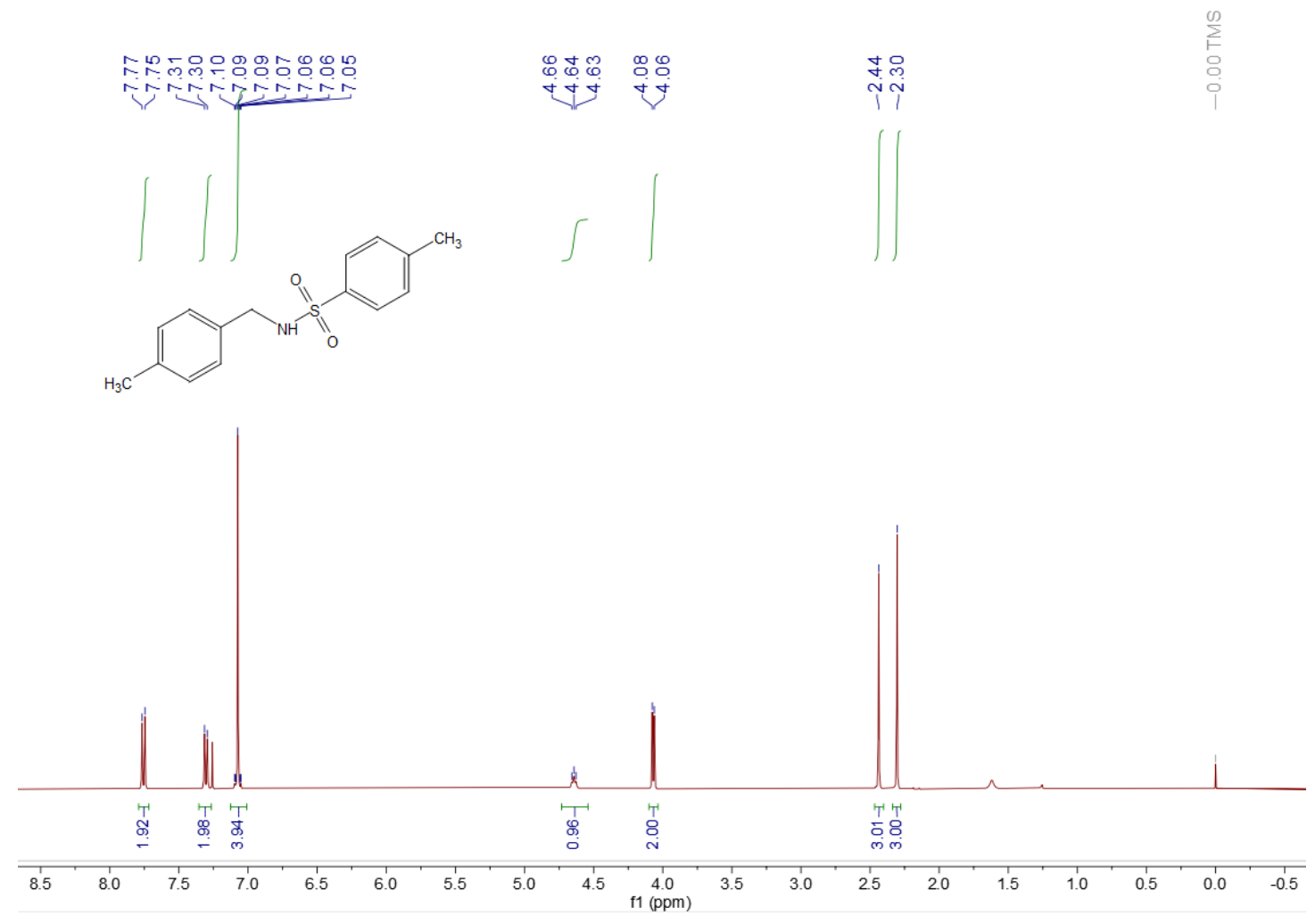

뚜요

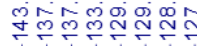

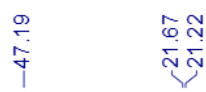<smiles>Cc1ccc(CCNS(=O)(=O)c2ccc(O)cc2)cc1</smiles>

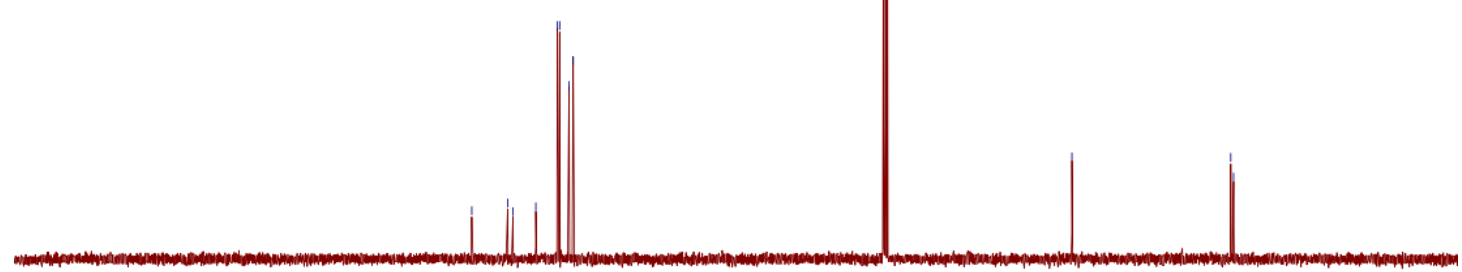

\begin{tabular}{llllllllllllllllllllllllll}
\hline 10 & 200 & 190 & 180 & 170 & 160 & 150 & 140 & 130 & 120 & 110 & 100 & 90 & 80 & 70 & 60 & 50 & 40 & 30 & 20 & 10 & 0 & -10
\end{tabular} 


\section{Compound 5ia}
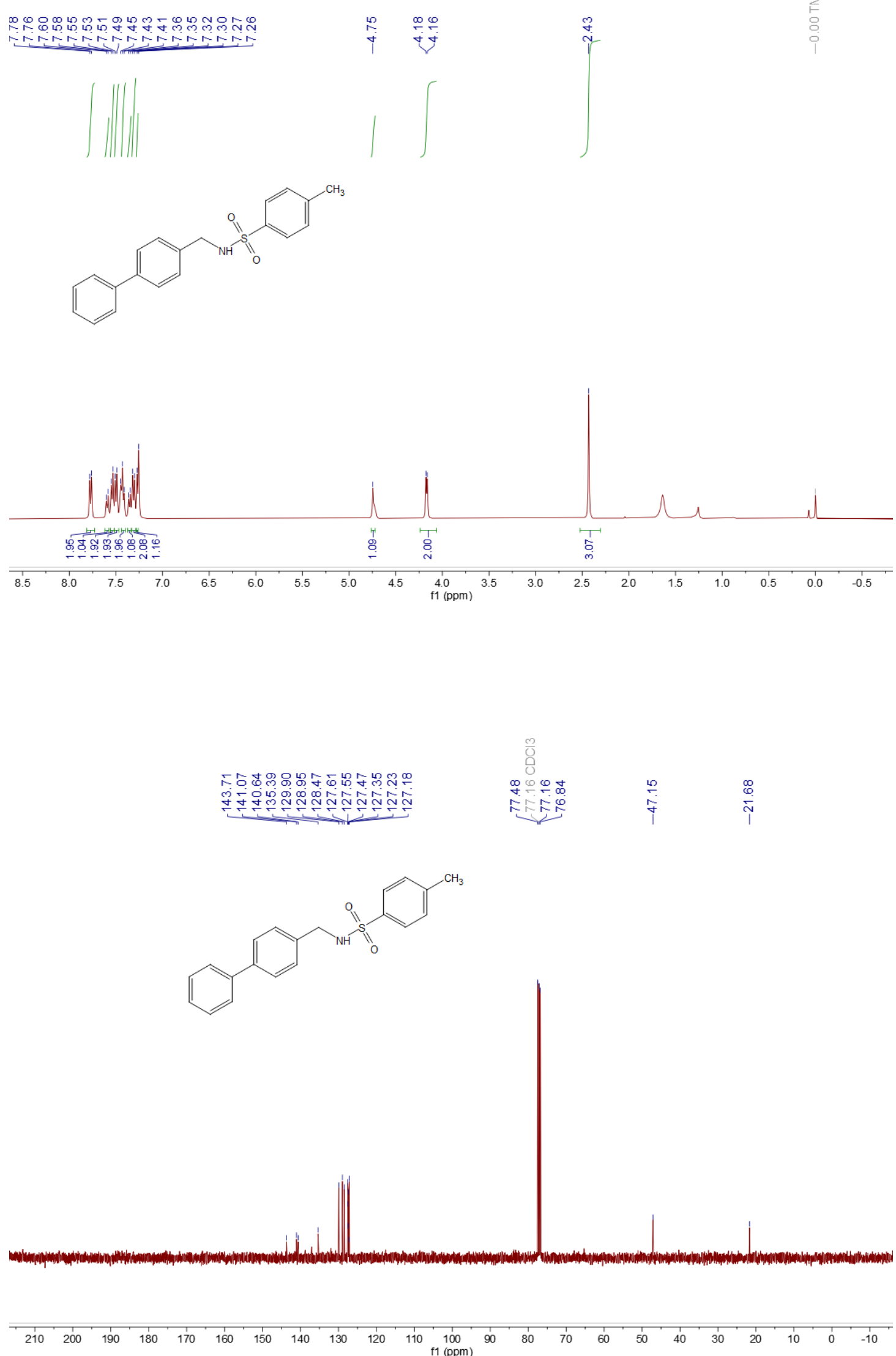


\section{Compound 5ja}

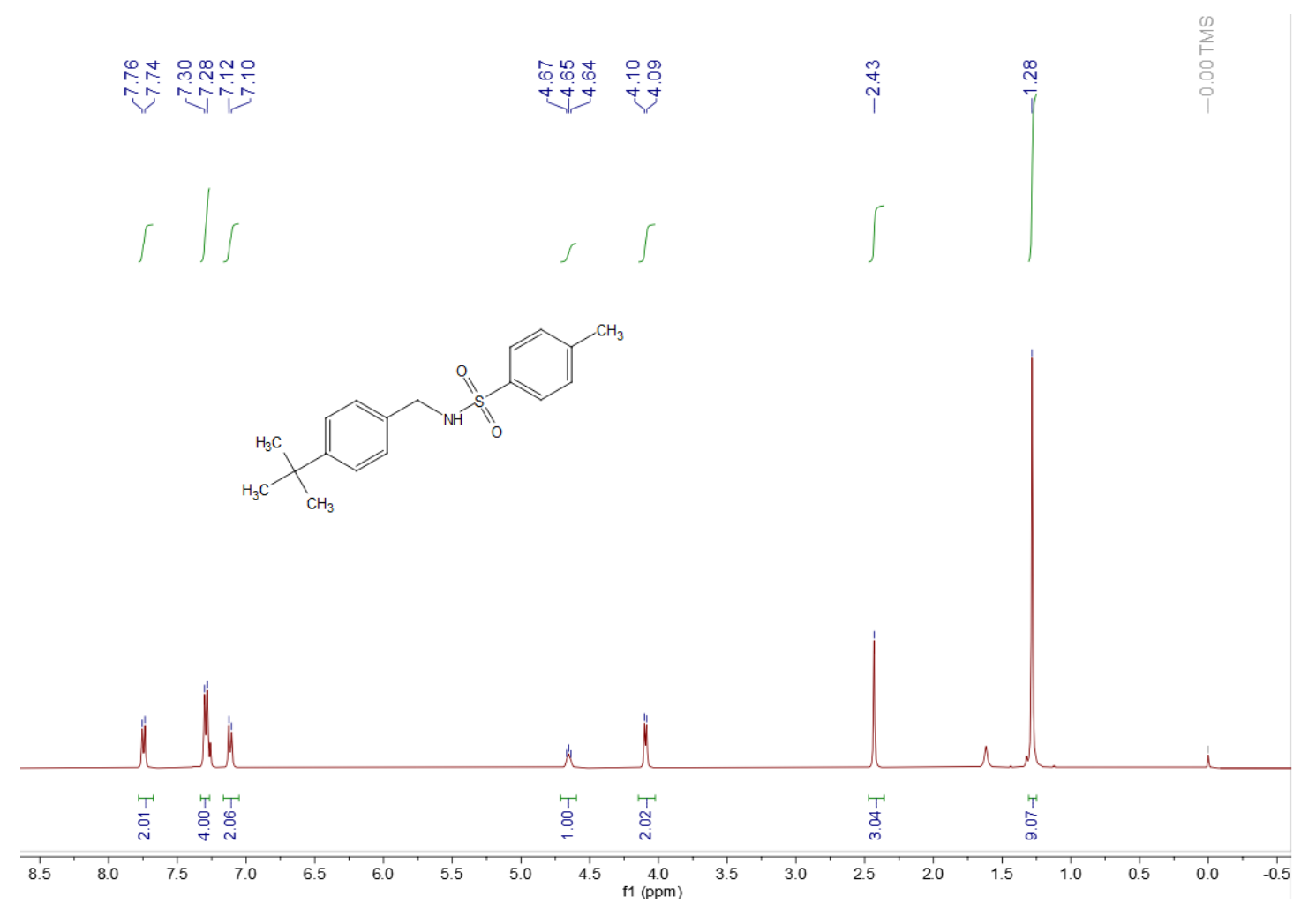

잉

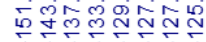

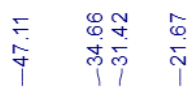

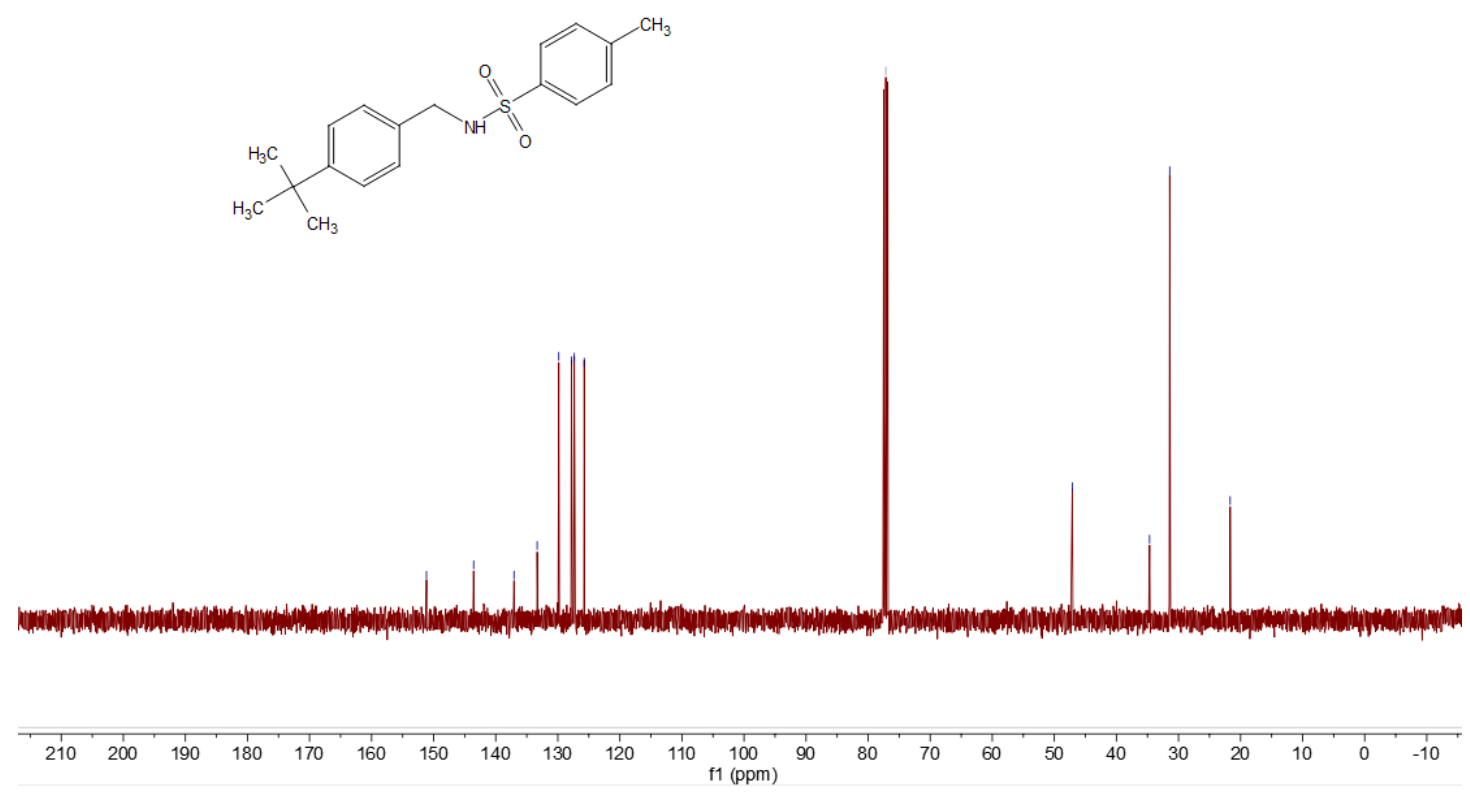




\section{Compound 5ka}

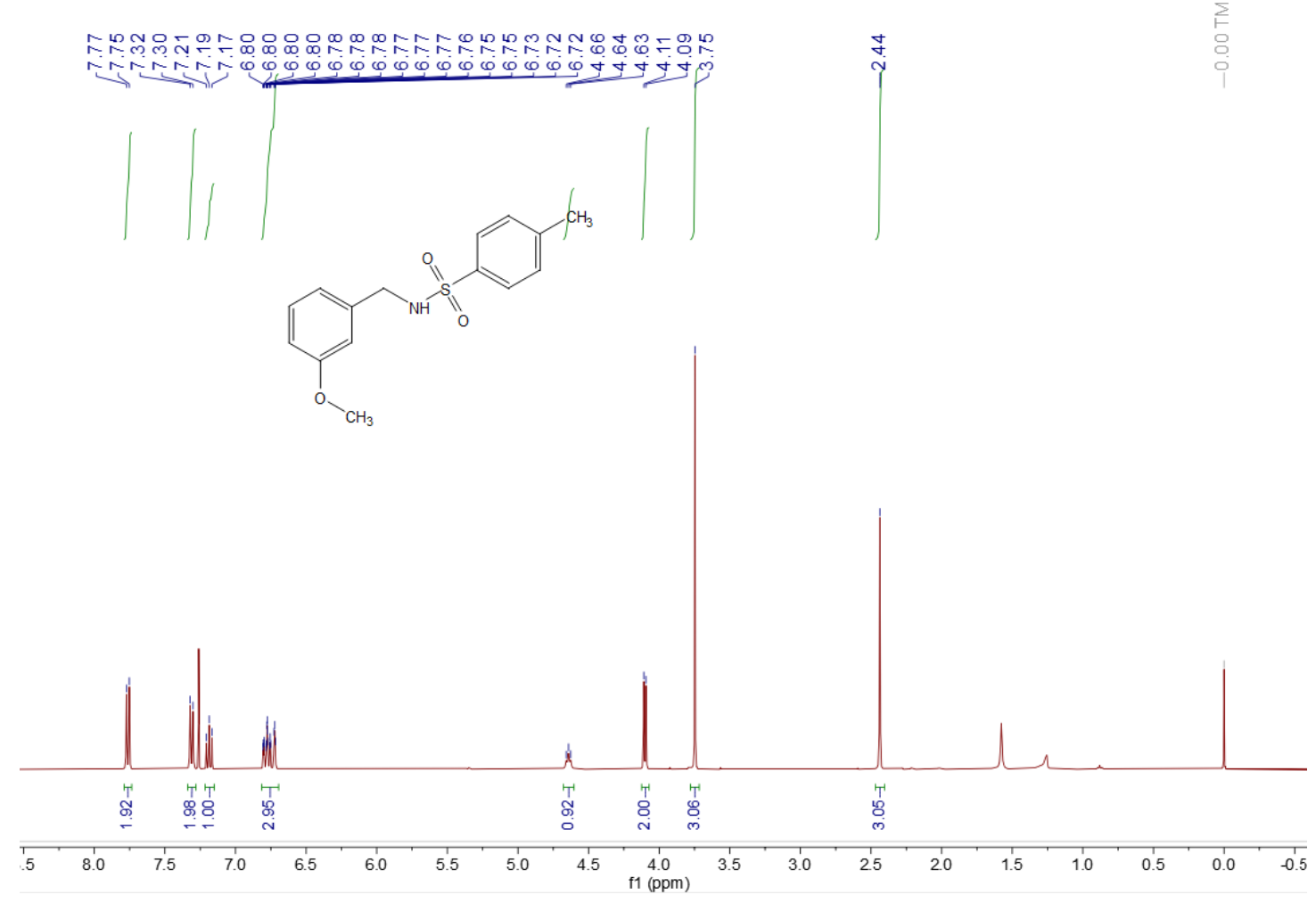

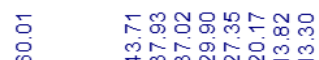

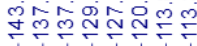

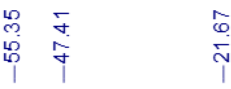

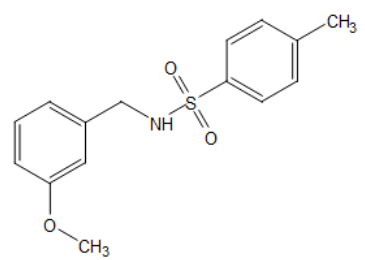

$\begin{array}{lllllllllllllllllllllll}1 & 1 \\ 210 & 200 & 190 & 180 & 170 & 160 & 150 & 140 & 130 & 120 & 110 & 100 & 90 & 80 & 70 & 60 & 50 & 40 & 30 & 20 & 10 & 0 & -10\end{array}$ 


\section{Compound 5la}
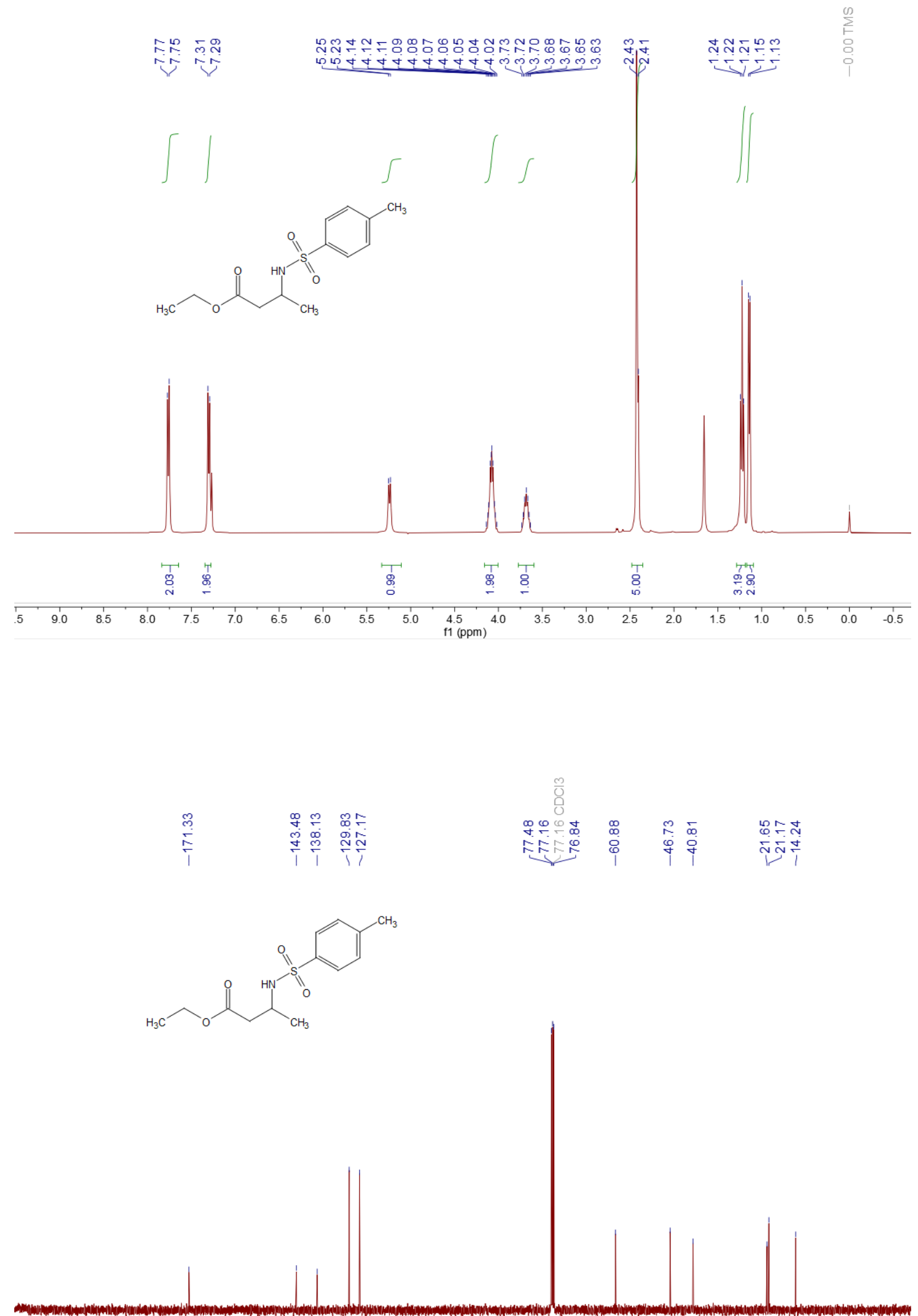

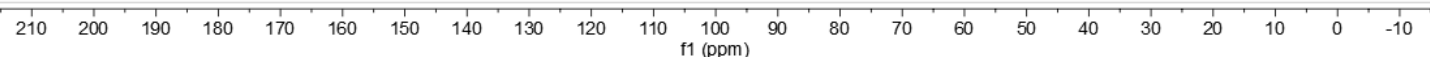




\section{Compound 5ma}

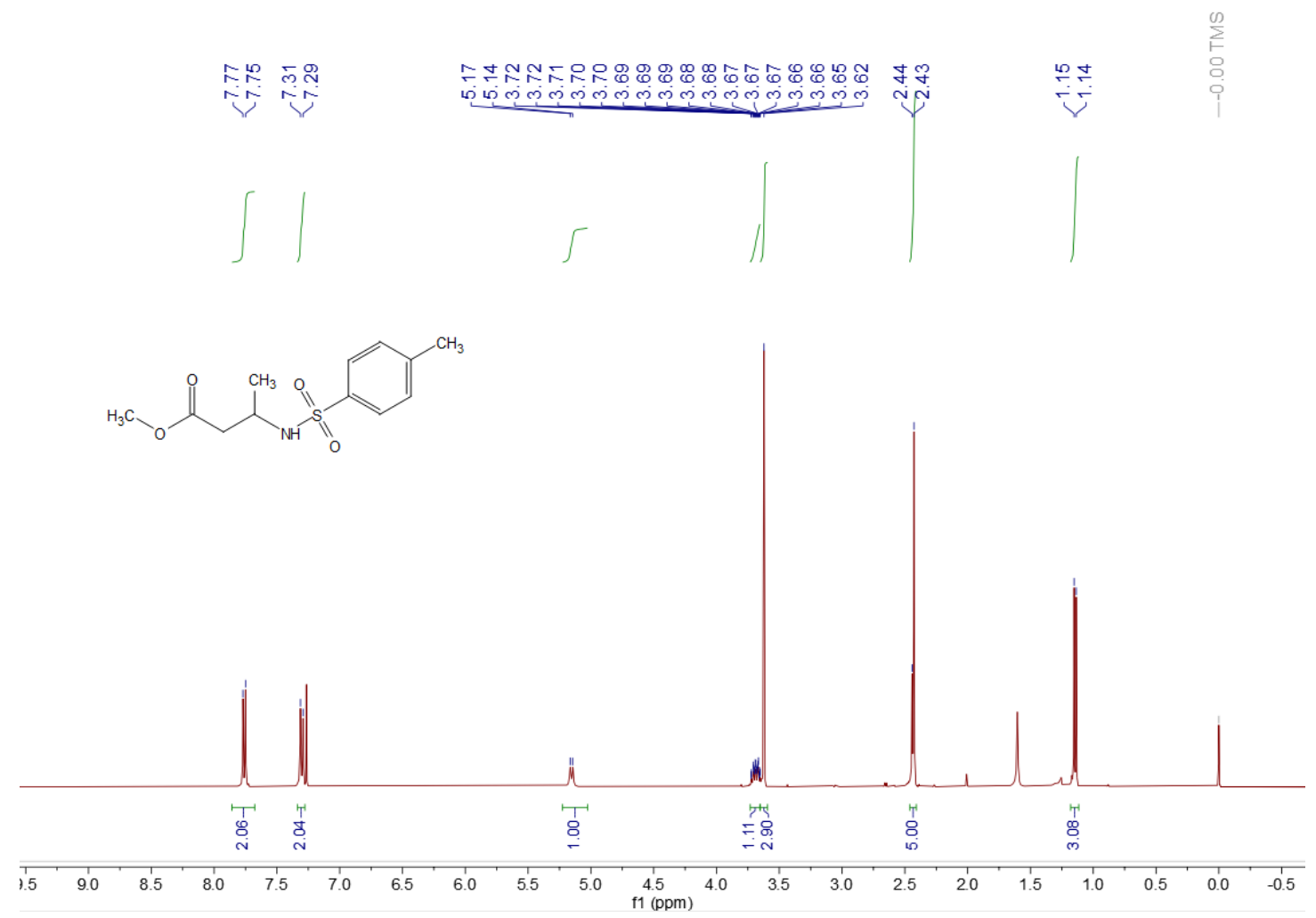

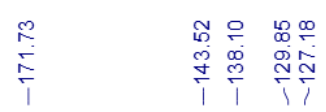

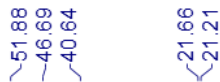<smiles>COC(=O)CC(C)NS(=O)(=O)c1ccc(C)cc1</smiles>

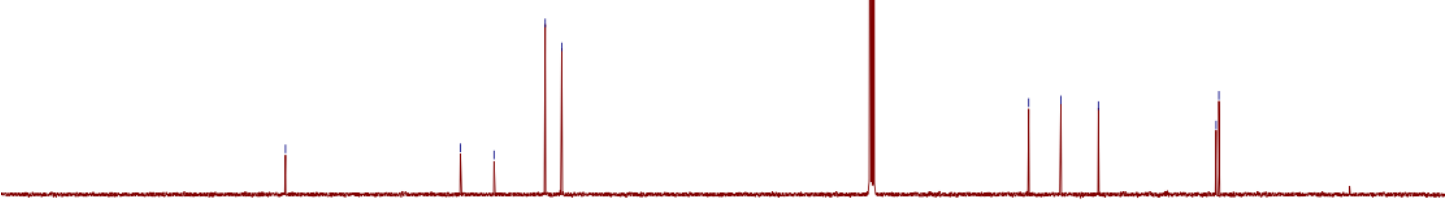




\section{Compound 5na}
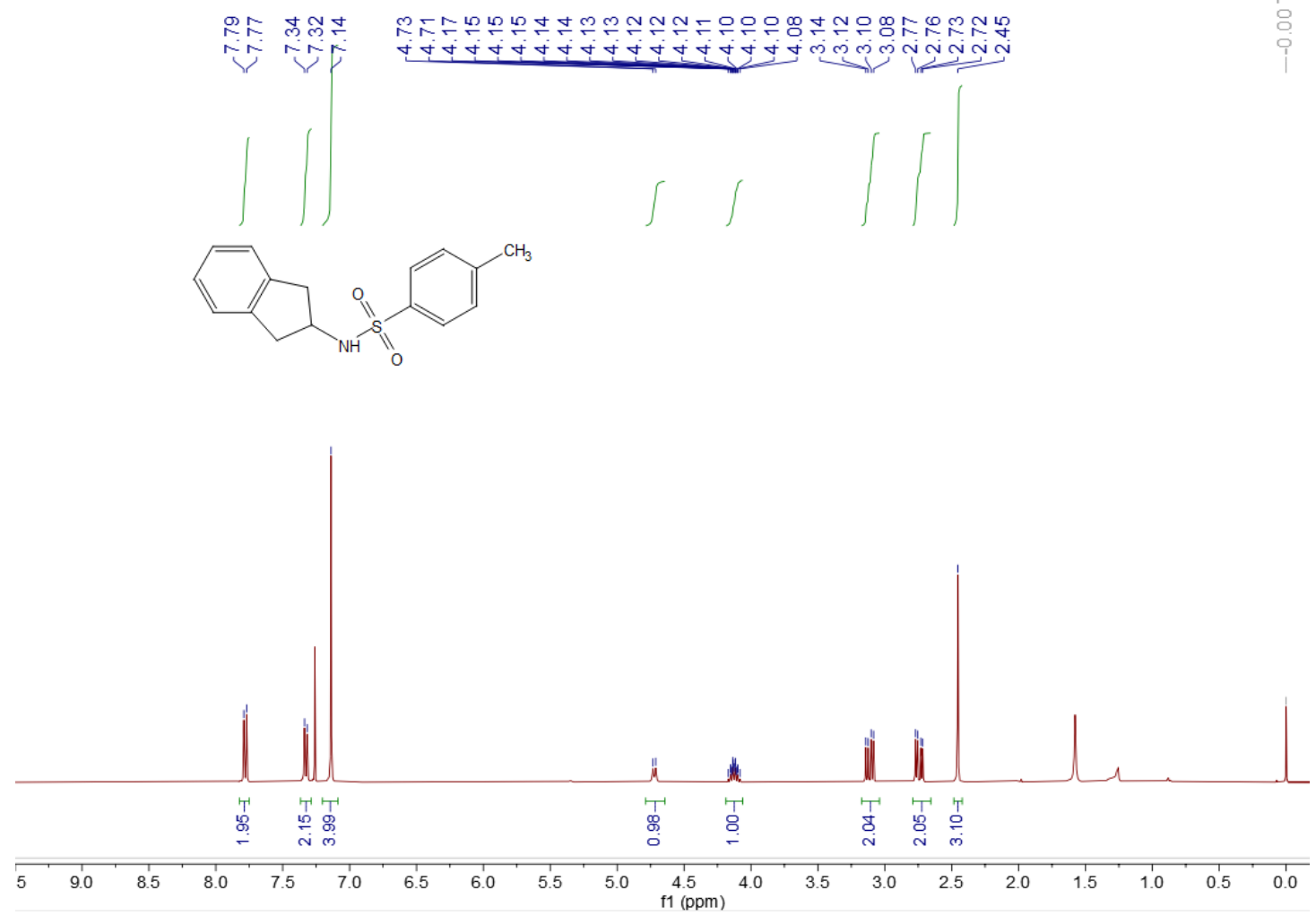

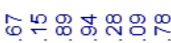

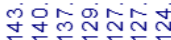

$\frac{8}{8}$

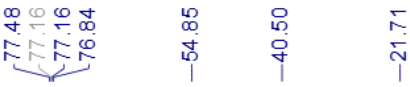
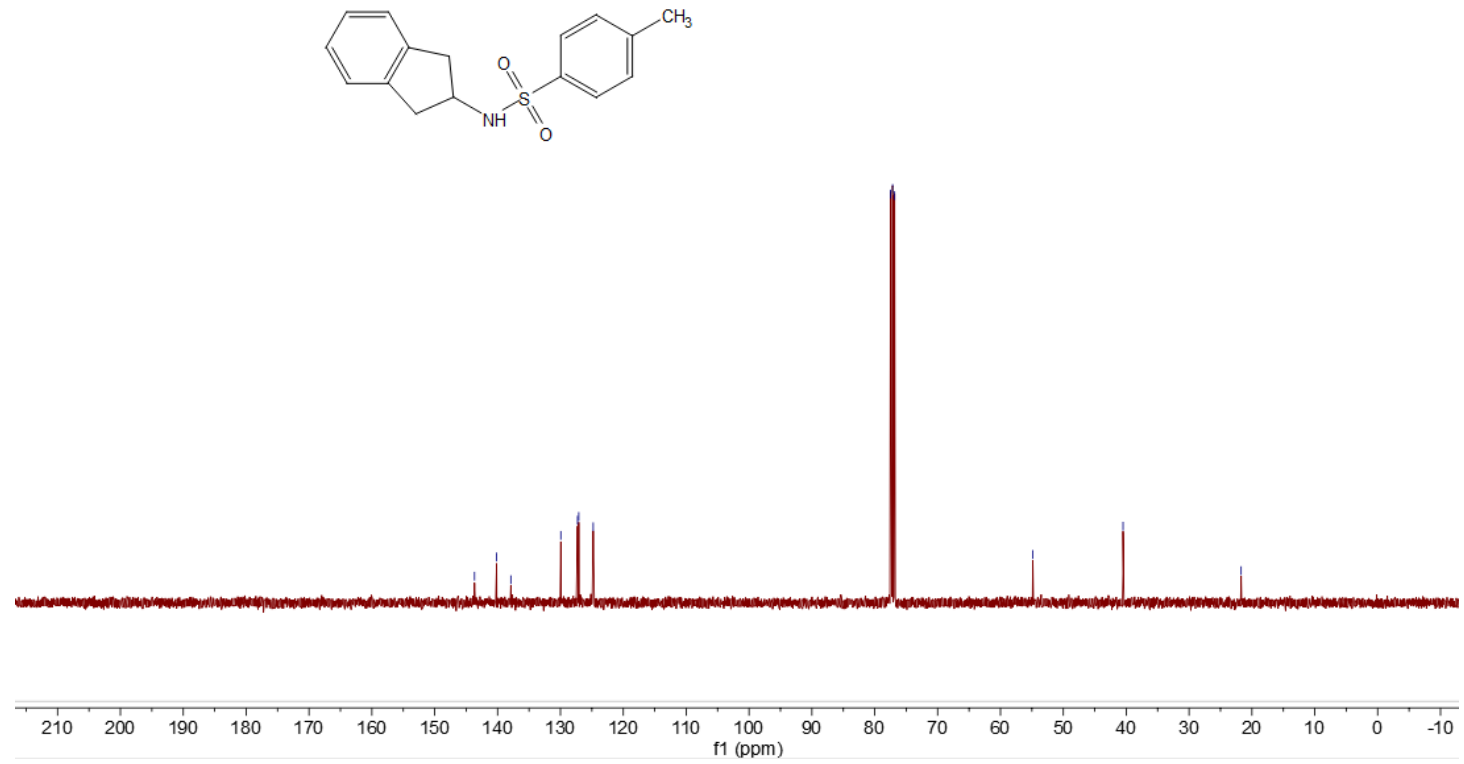


\section{Compound 5oa}

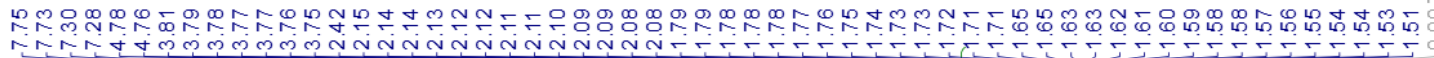<smiles>C1=CCC1</smiles><smiles>O=S(=O)(NC1CCC1)c1ccc(O)cc1</smiles>
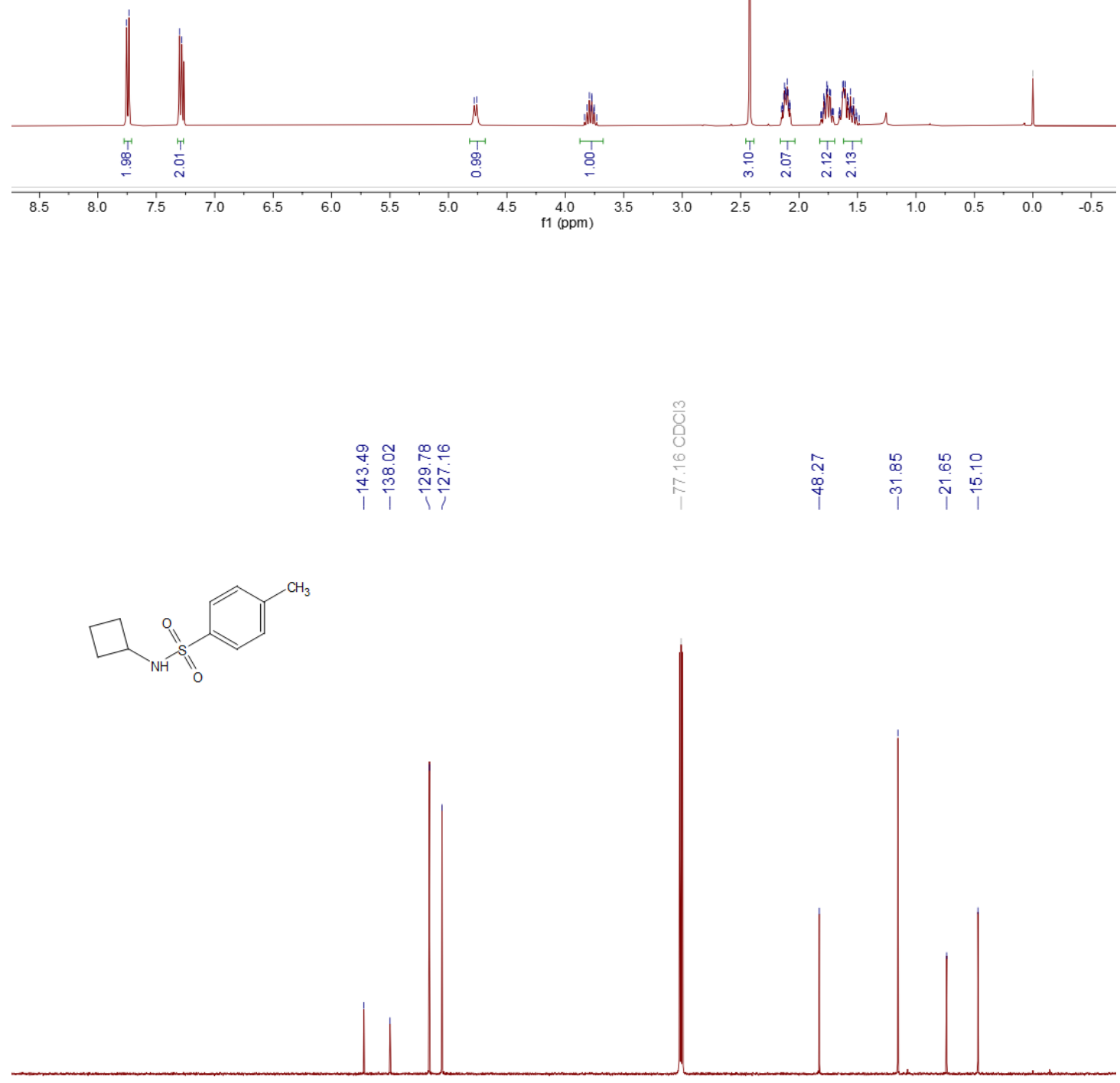

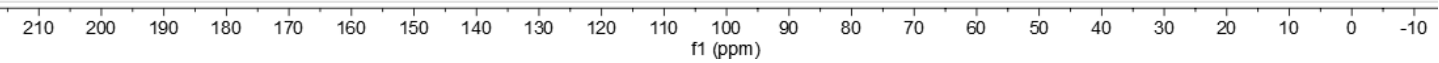




\section{Compound 5pa}

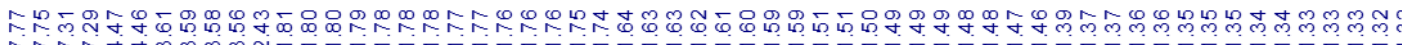

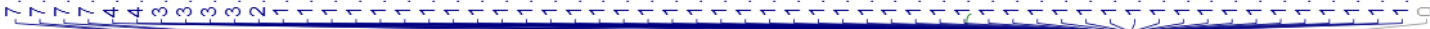

$\int$
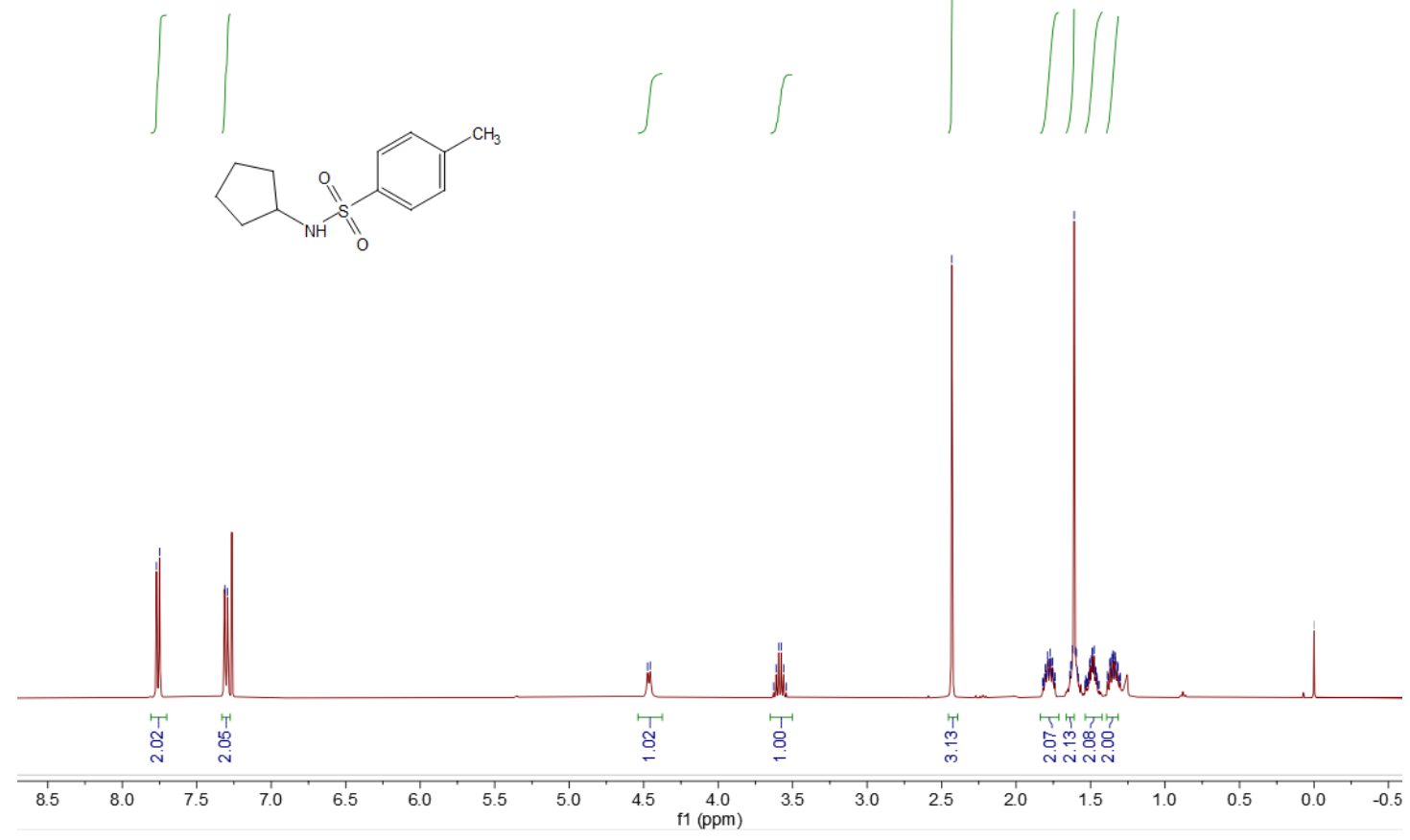

d) 8 gก

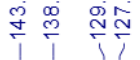

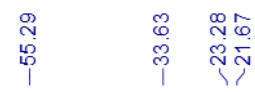
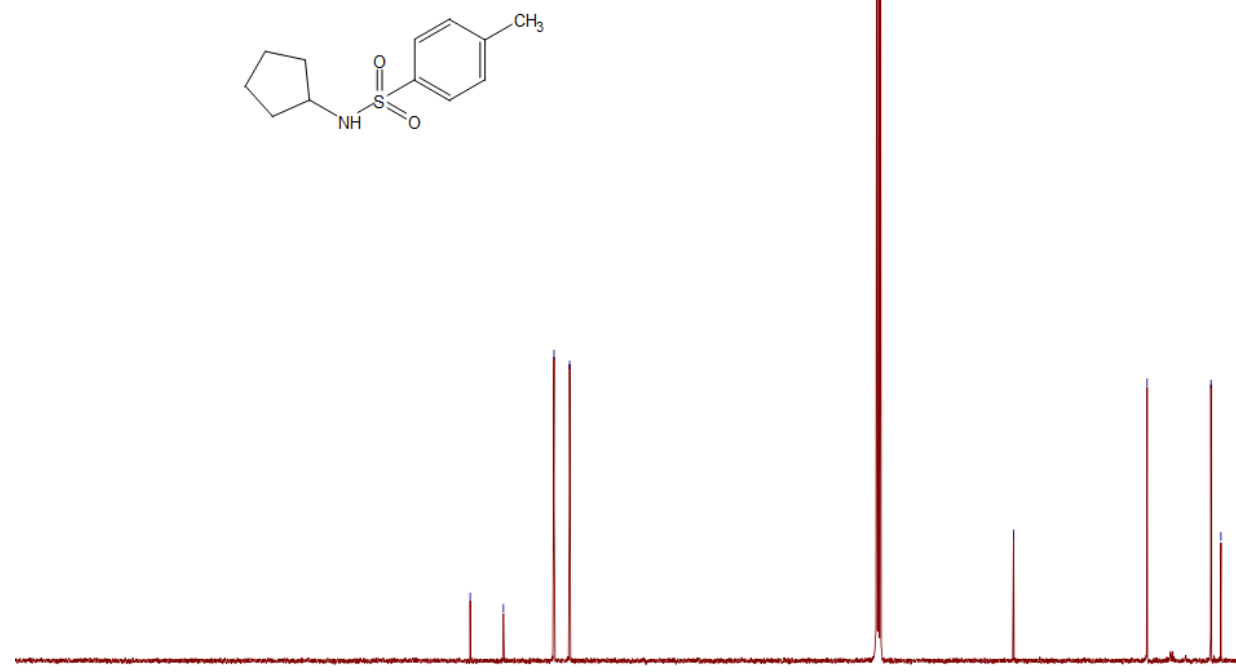

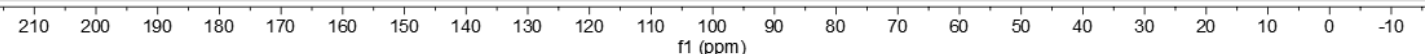




\section{Compound 5qa}
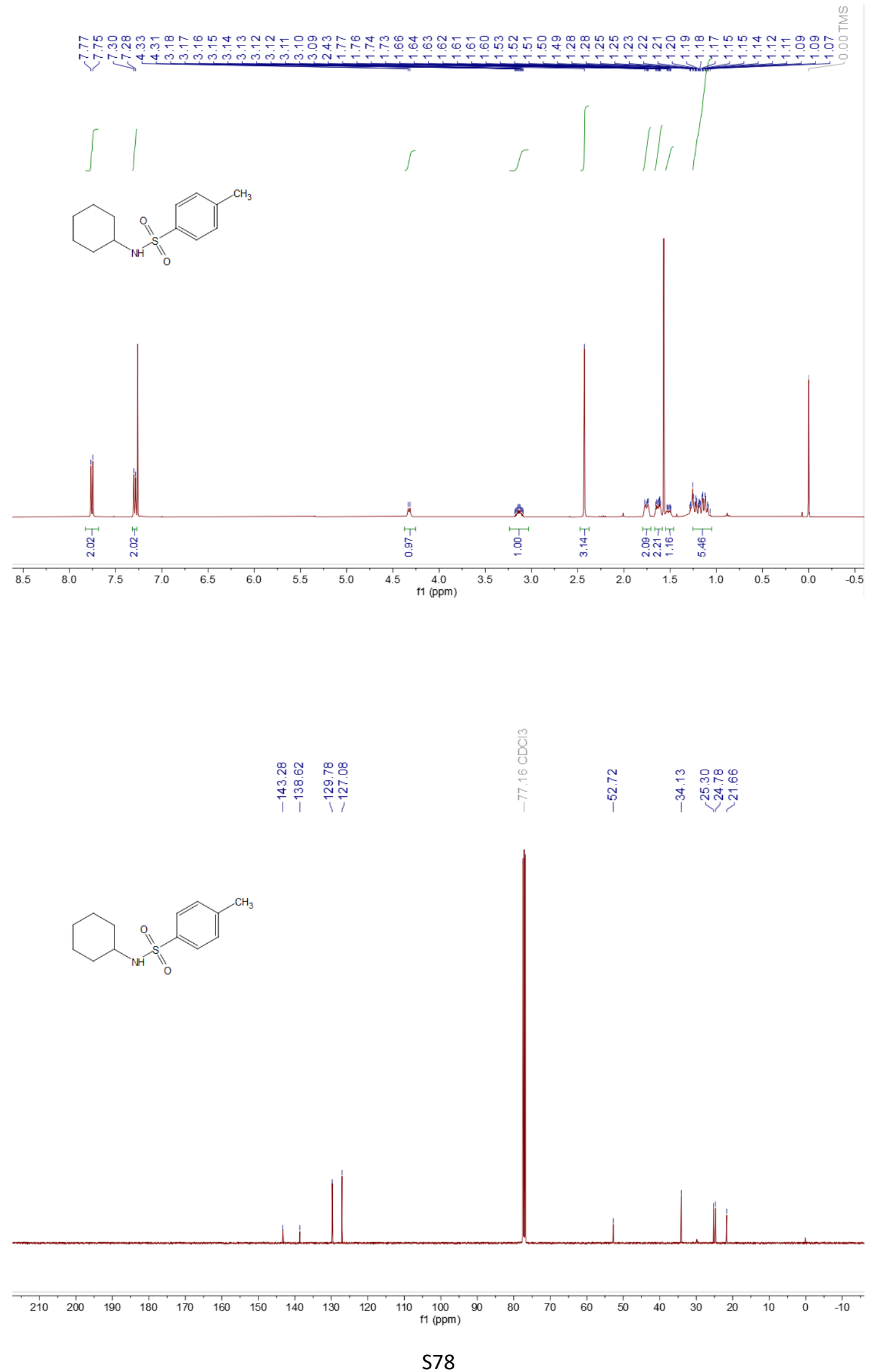


\section{Compound 3ab}

œ

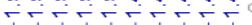

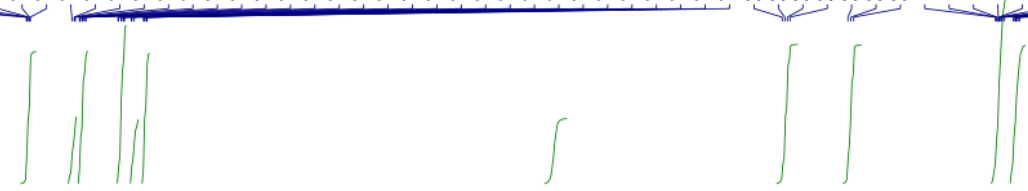<smiles>O=S(=O)(NCCCCc1ccccc1)c1ccccc1</smiles>
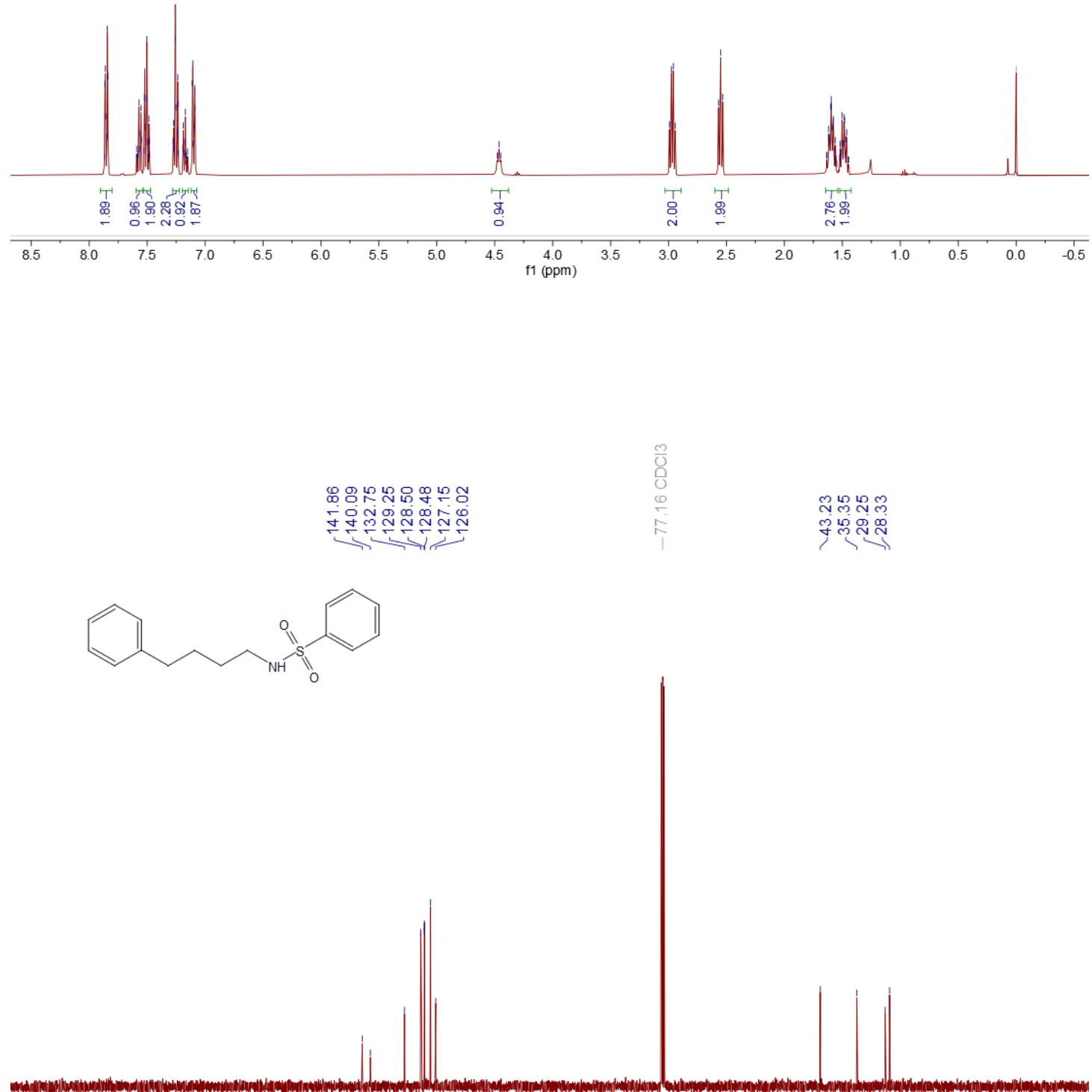

$\begin{array}{llllllllllllllllllllllllllllllllll} & 210 & 200 & 190 & 180 & 170 & 160 & 150 & 140 & 130 & 120 & 110 & 100 & 90 & 80 & 70 & 60 & 50 & 40 & 30 & 20 & 10 & 0 & -10\end{array}$ 


\section{Compound 3ac}

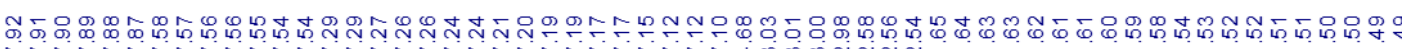

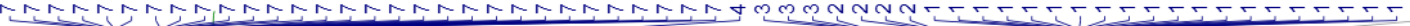
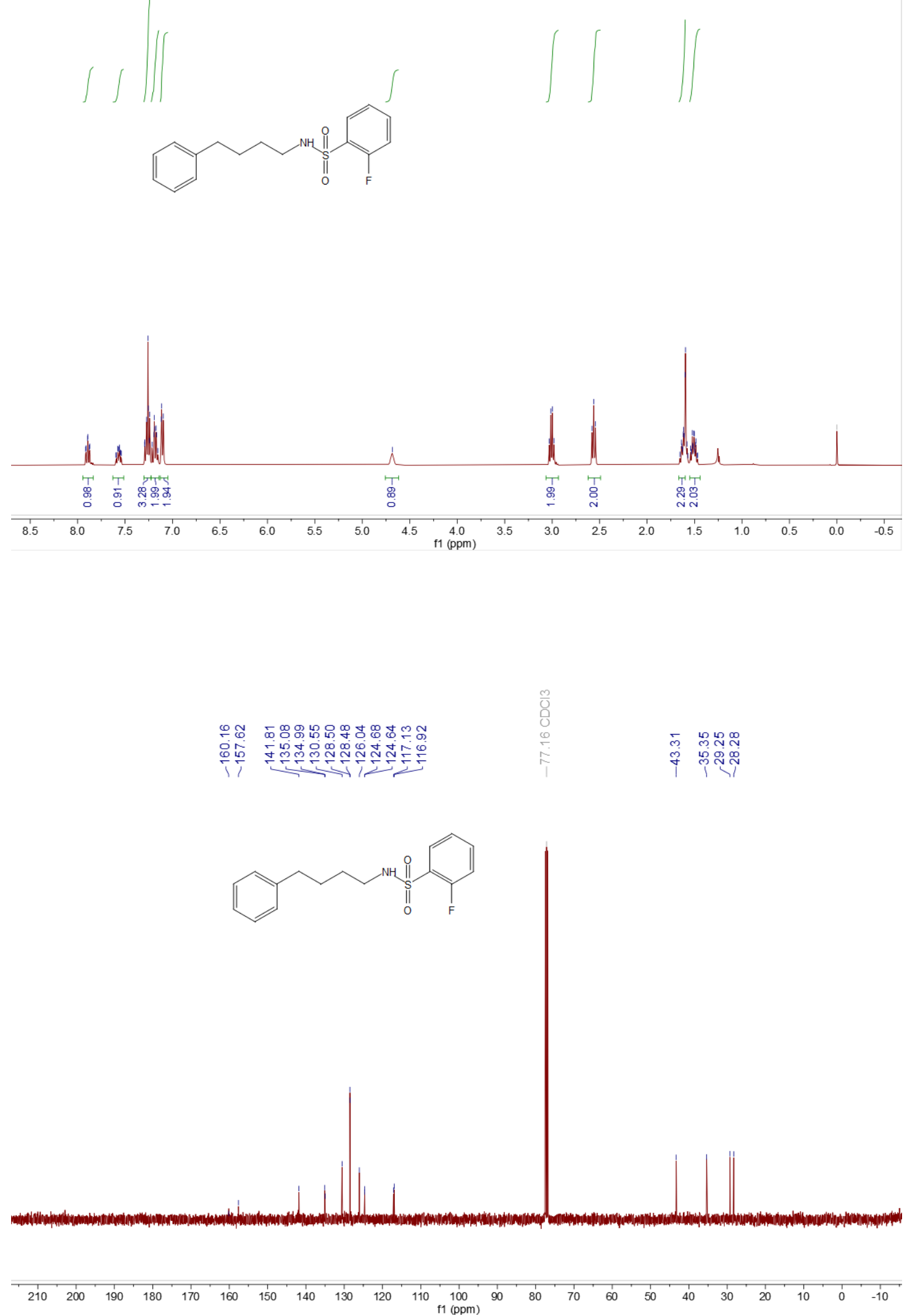


\section{Compound 3ad}

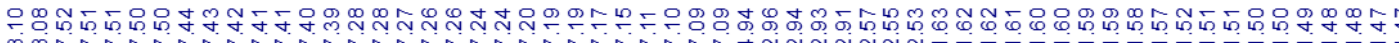
NMNRNNNMNRN
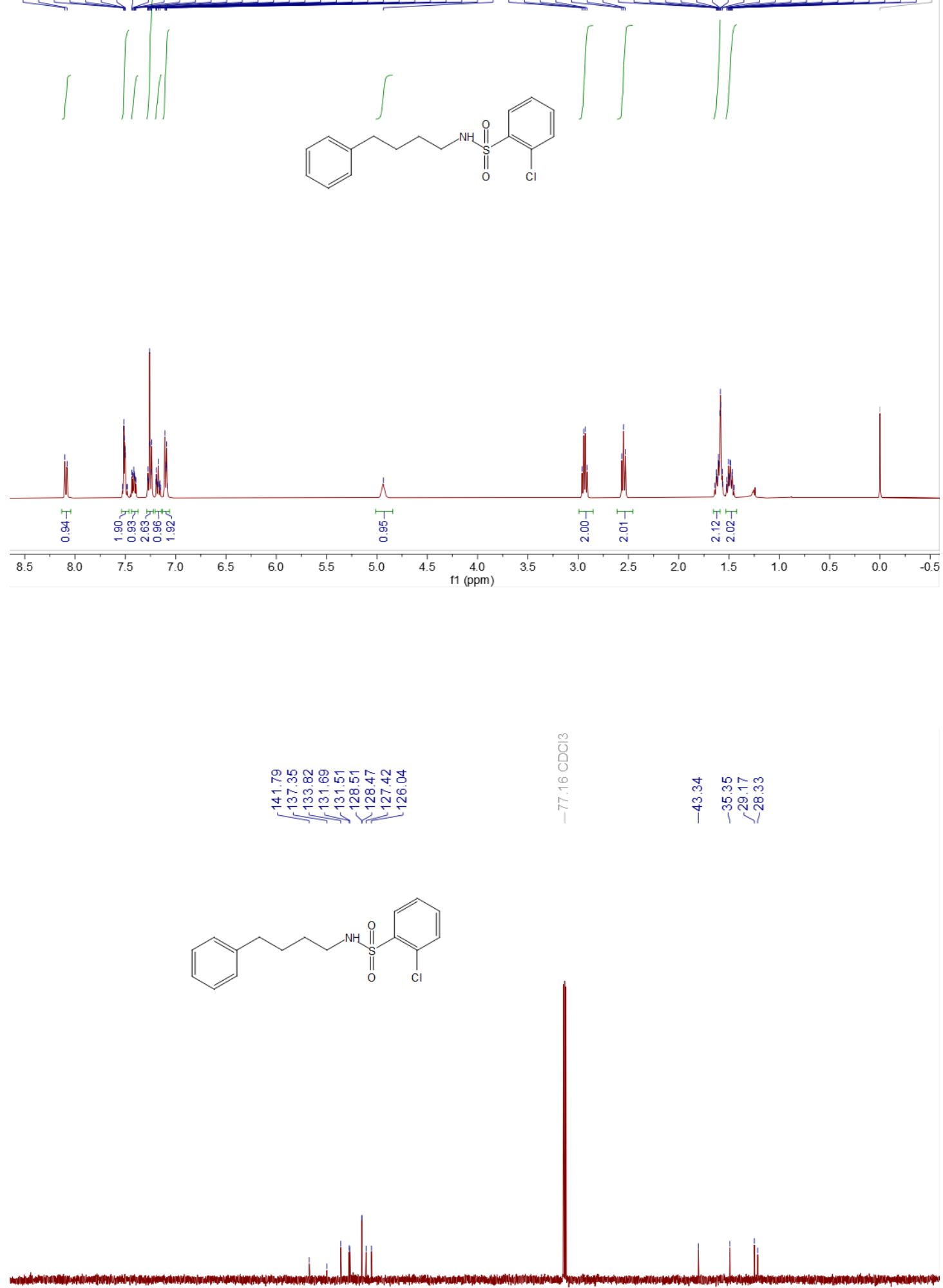


\section{Compound 3ae}
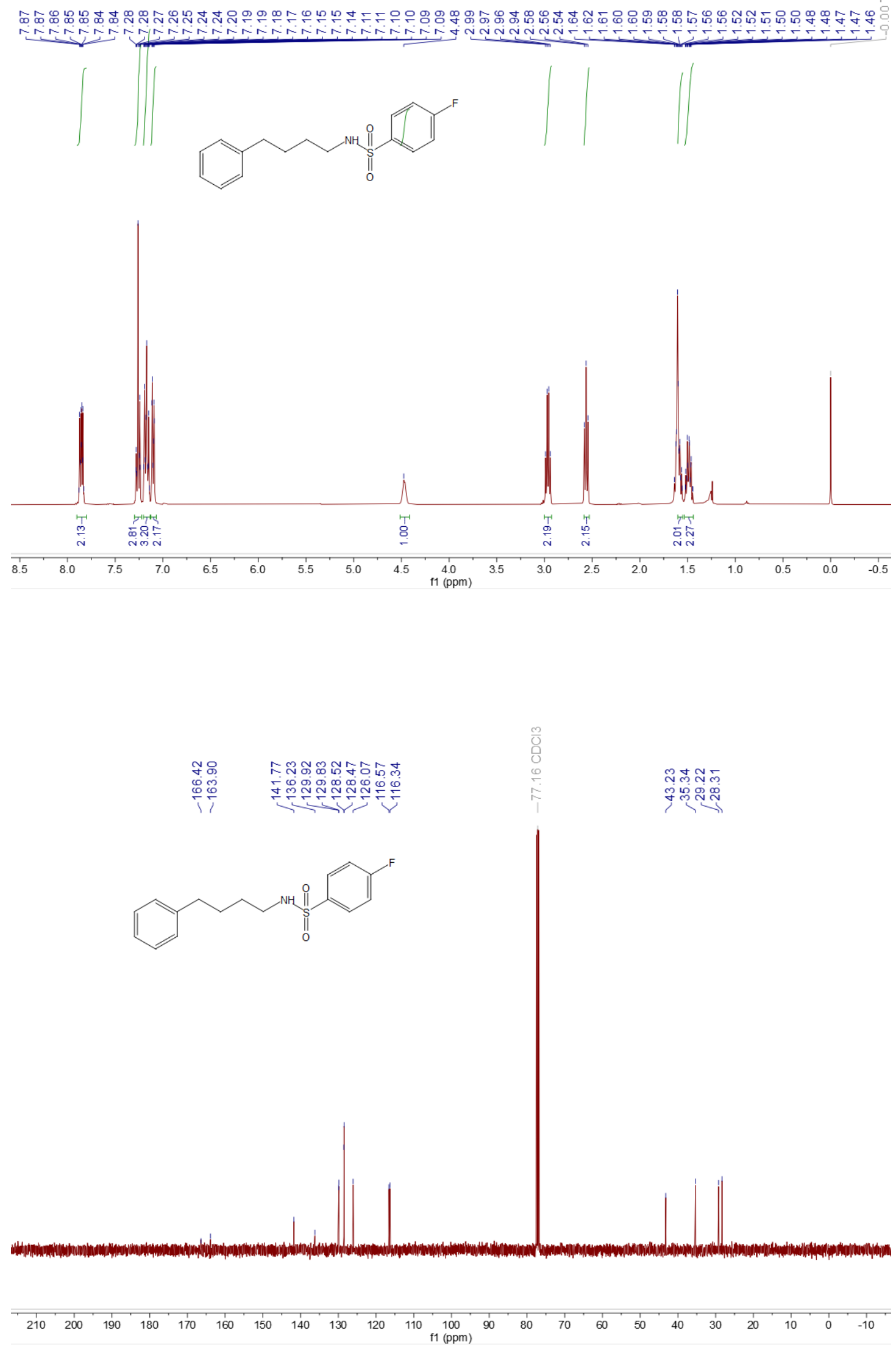


\section{Compound 3af}

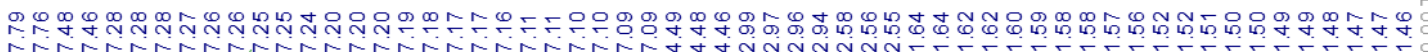
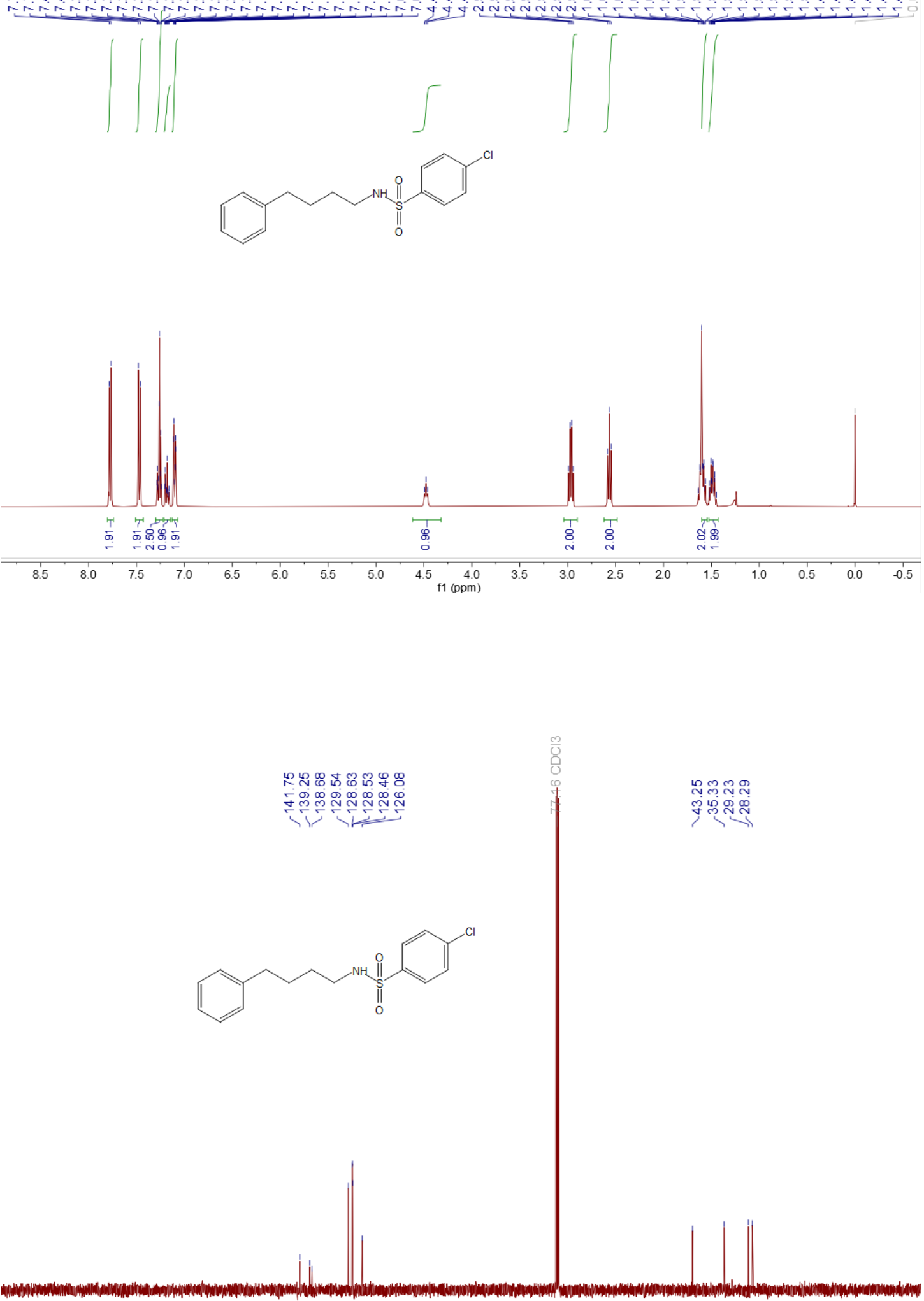

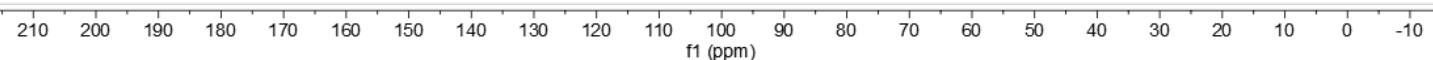




\section{Compound 3ag}

Г అ

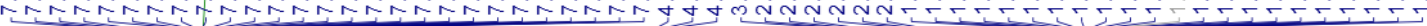

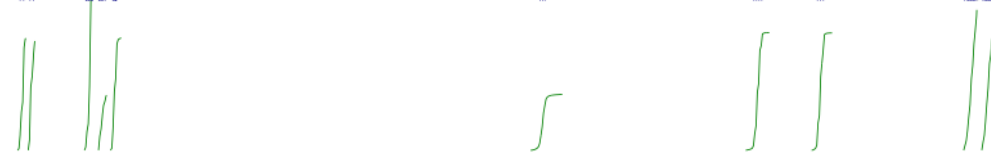<smiles>O=S(=O)(NCCCCc1ccccc1)c1ccc(Br)cc1</smiles>

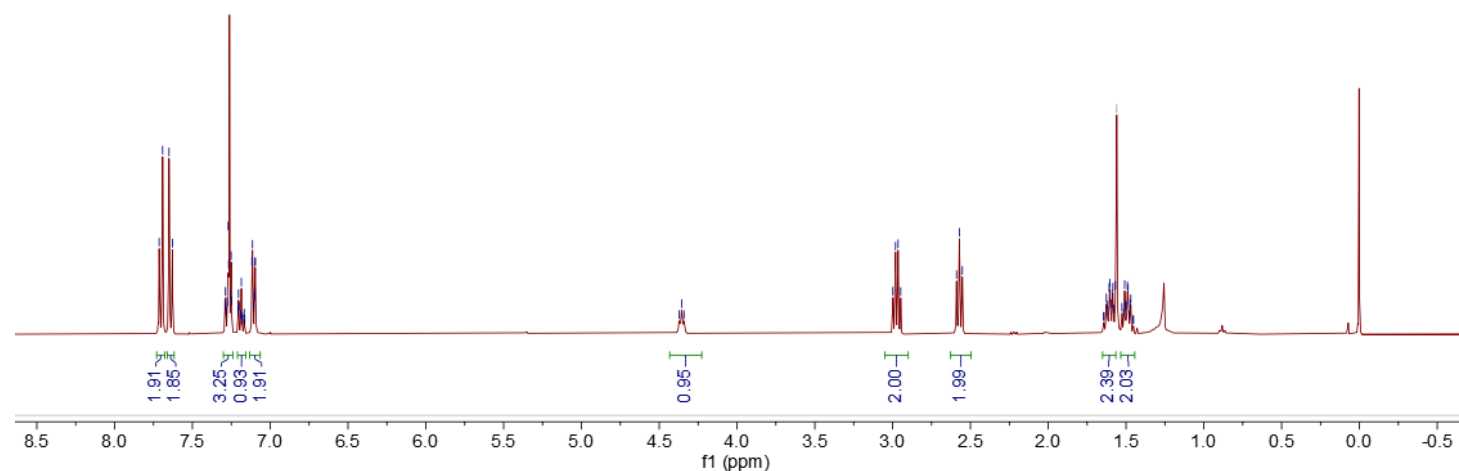

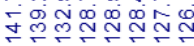

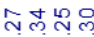<smiles>O=S(=O)(NCCCCc1ccccc1)c1ccc(Br)cc1</smiles>
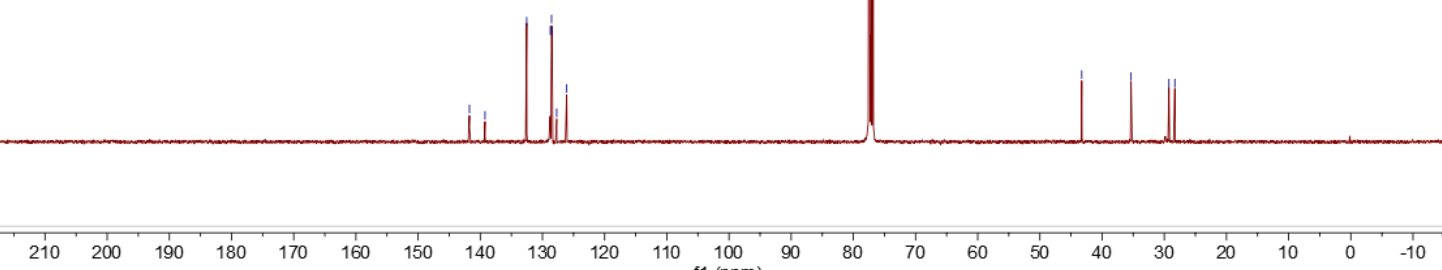


\section{Compound 3ah}

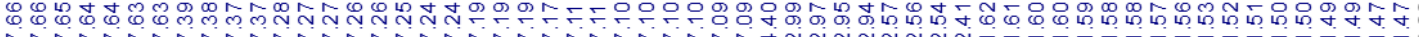
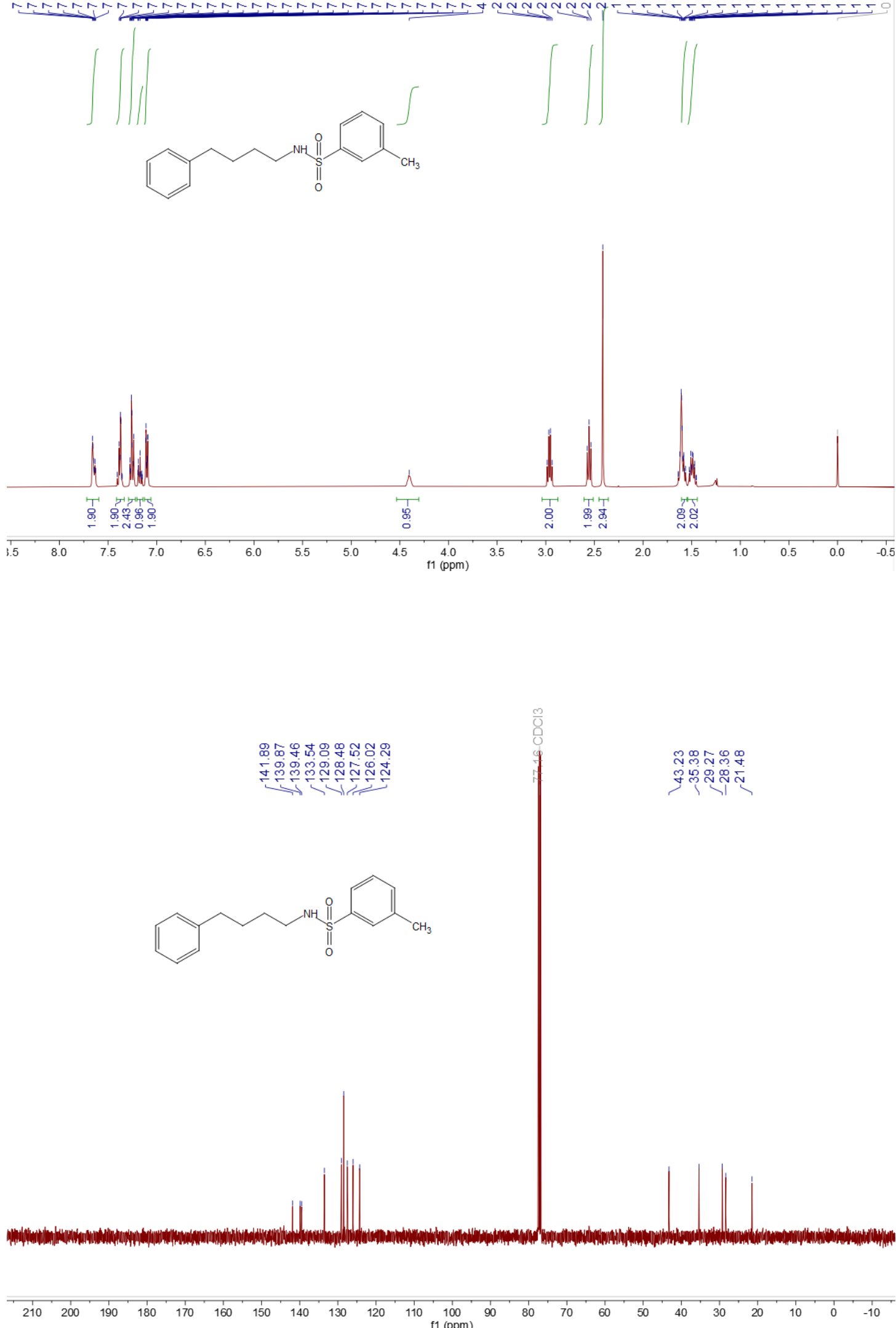


\section{Compound 3ai}
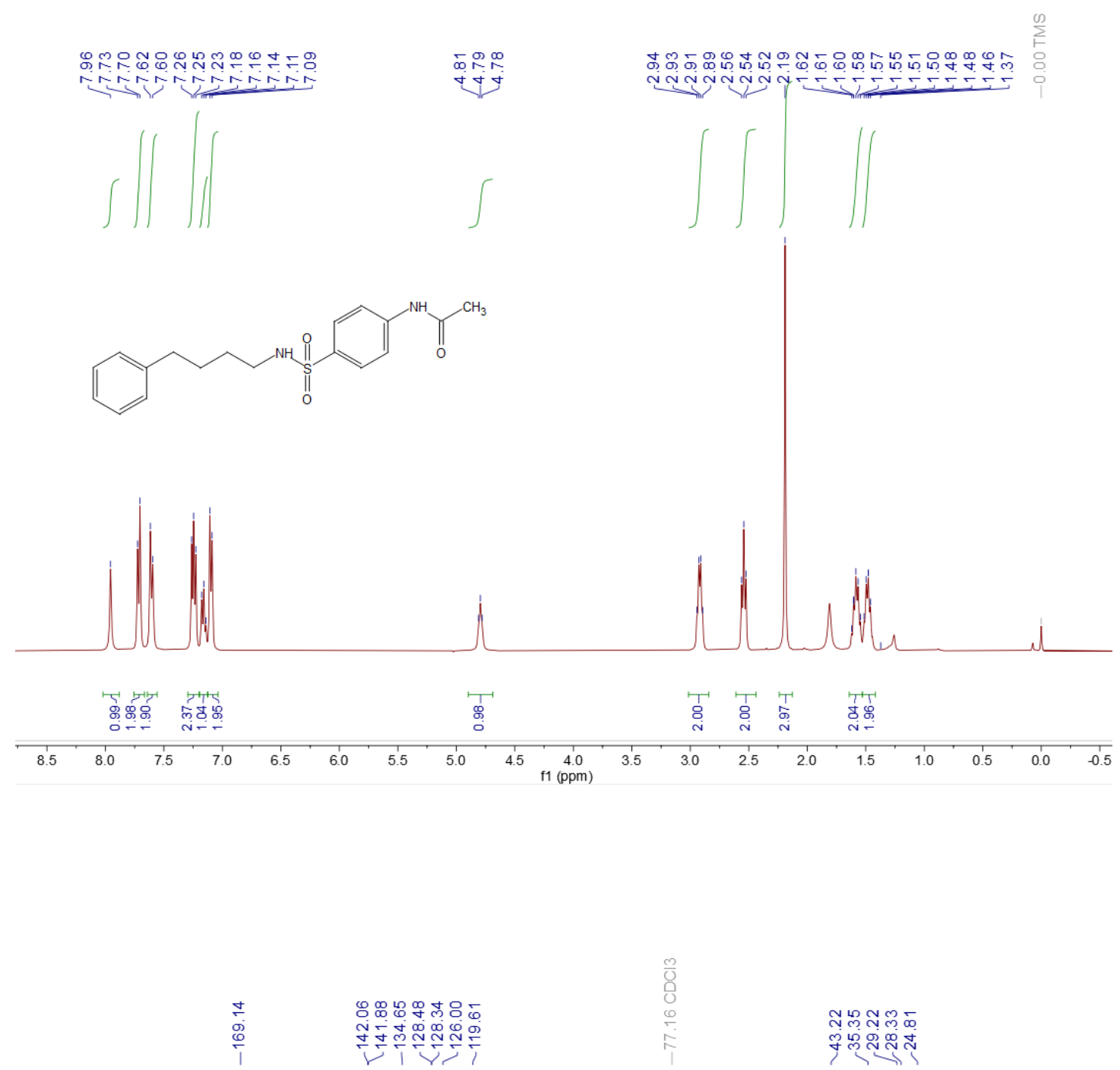

స్พำ

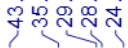
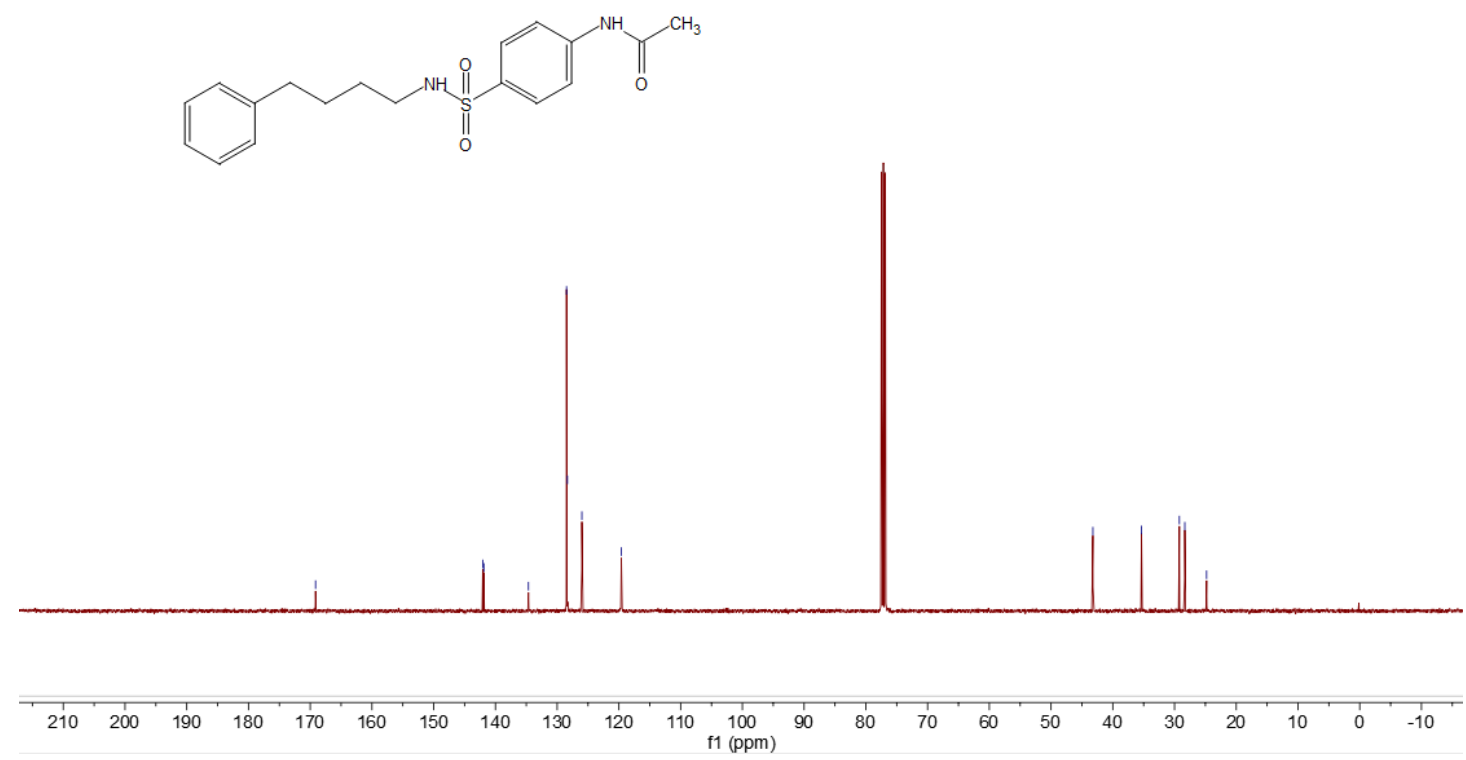


\section{Compound 3aj}

م

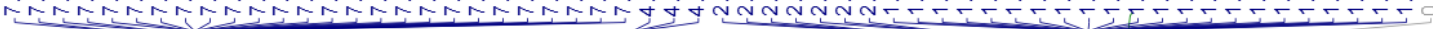

$\iiint d$<smiles>CC(C)(C)c1ccc(S(=O)(=O)NCCCCc2ccccc2)cc1</smiles>
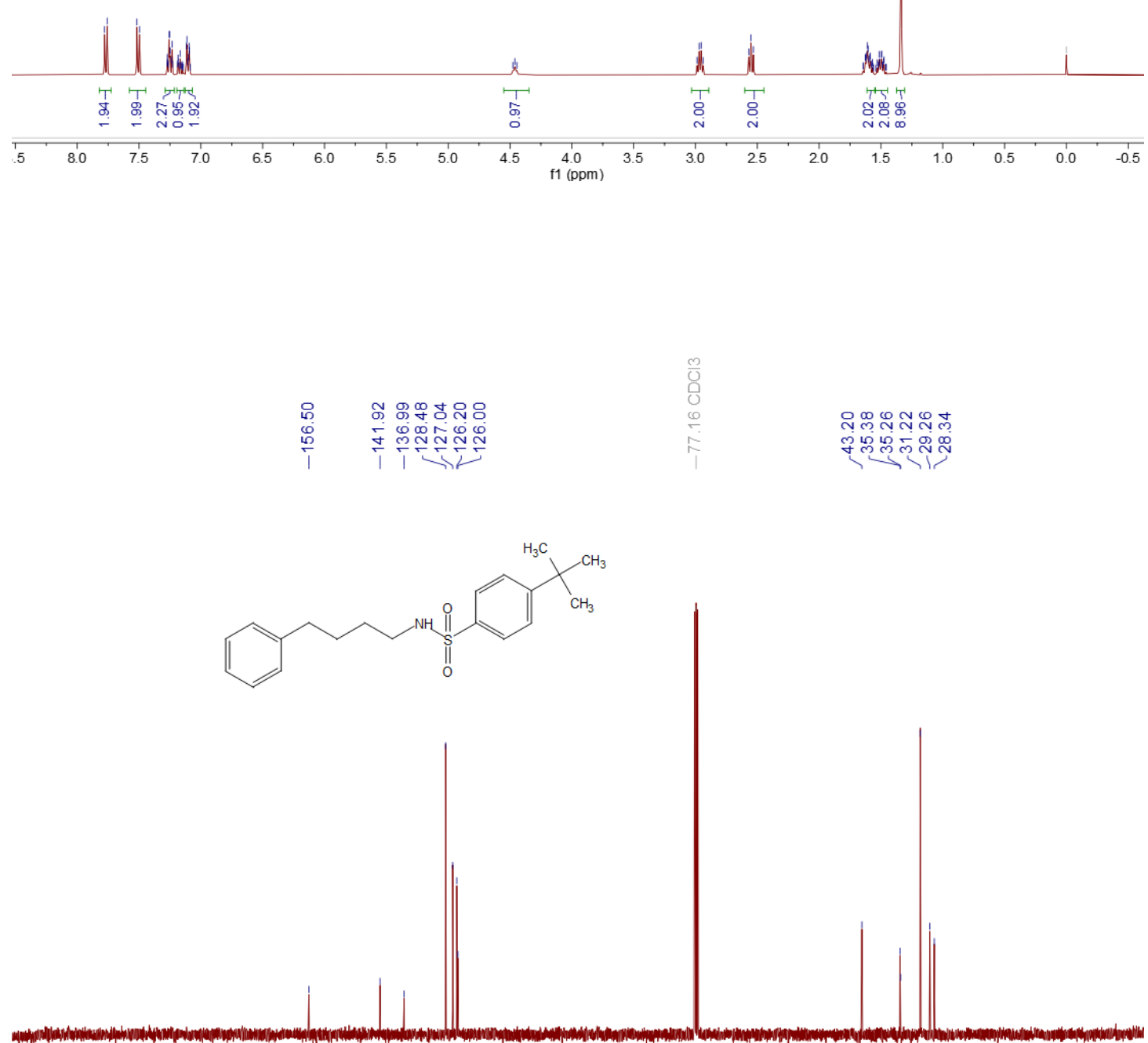

$\begin{array}{llllllllllll}210 & 200 & 190 & 180 & 170 & 160 & 150 & 140 & 130 & 120 & 110 & 100 \\ 11 & & & & & & \end{array}$ 


\section{Compound 3ak}

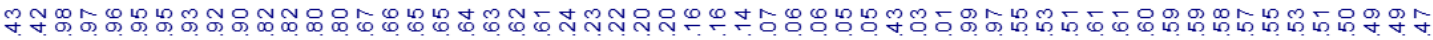

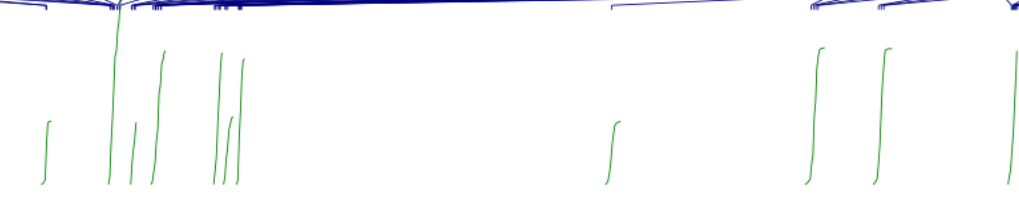<smiles>O=S(=O)(NCCCCc1ccccc1)c1ccc2ccccc2c1</smiles>
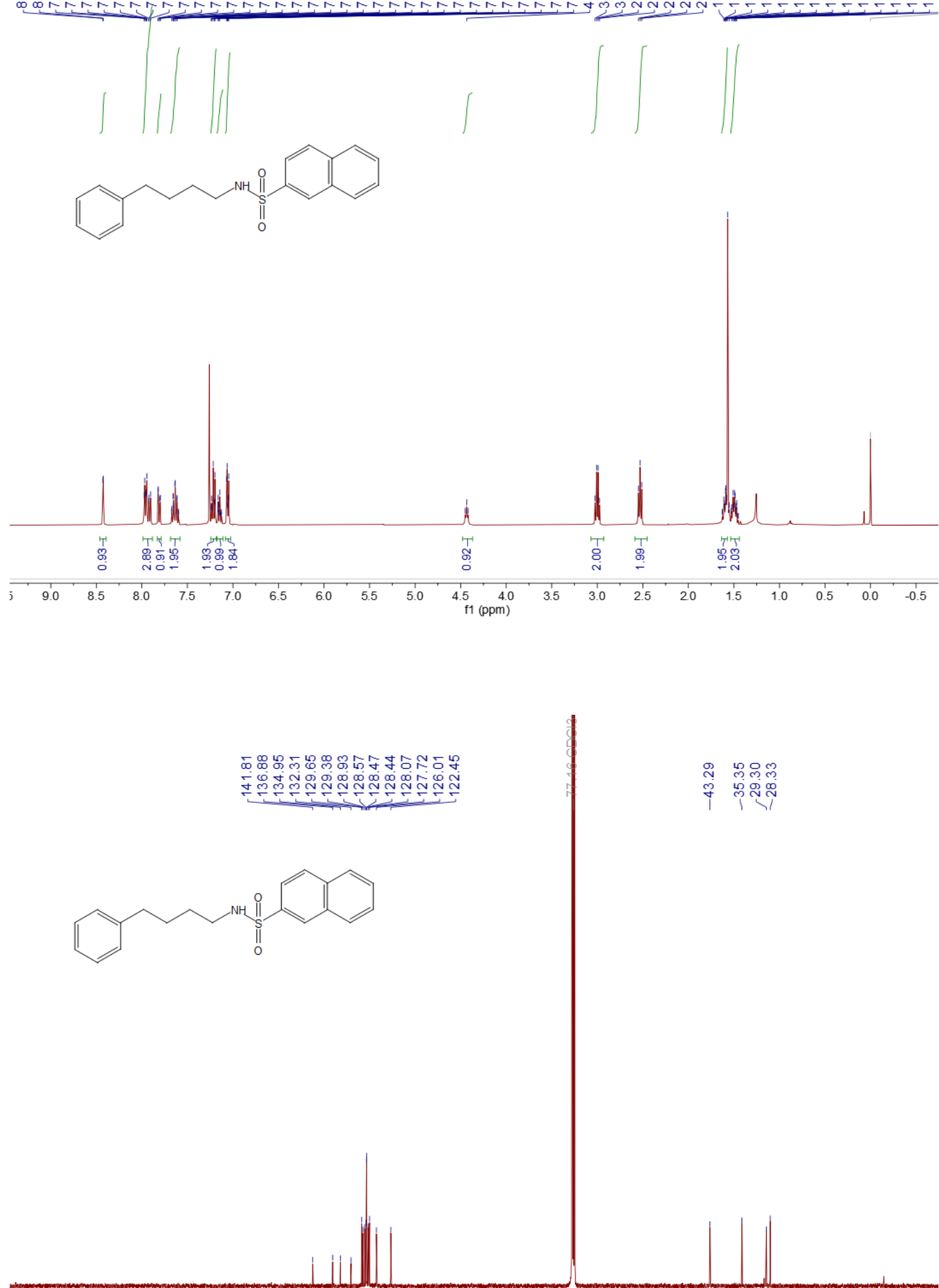

$\begin{array}{lllllllllllllllllllllllllllllll}1 & 210 & 200 & 190 & 180 & 170 & 160 & 150 & 140 & 130 & 120 & 110 & 100 & 90 & 80 & 70 & 60 & 50 & 40 & 30 & 20 & 10 & 0 & -10\end{array}$ 


\section{Compound 3al}

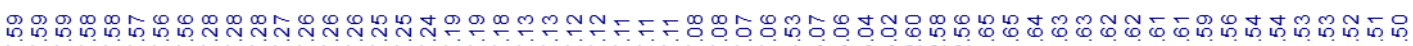

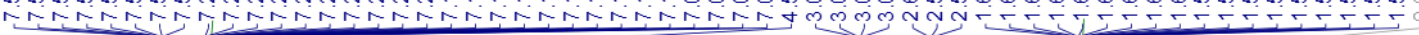

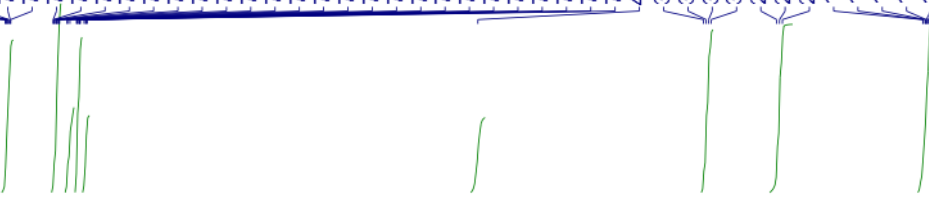<smiles>O=S(=O)(NCCCCc1ccccc1)c1cccs1</smiles>
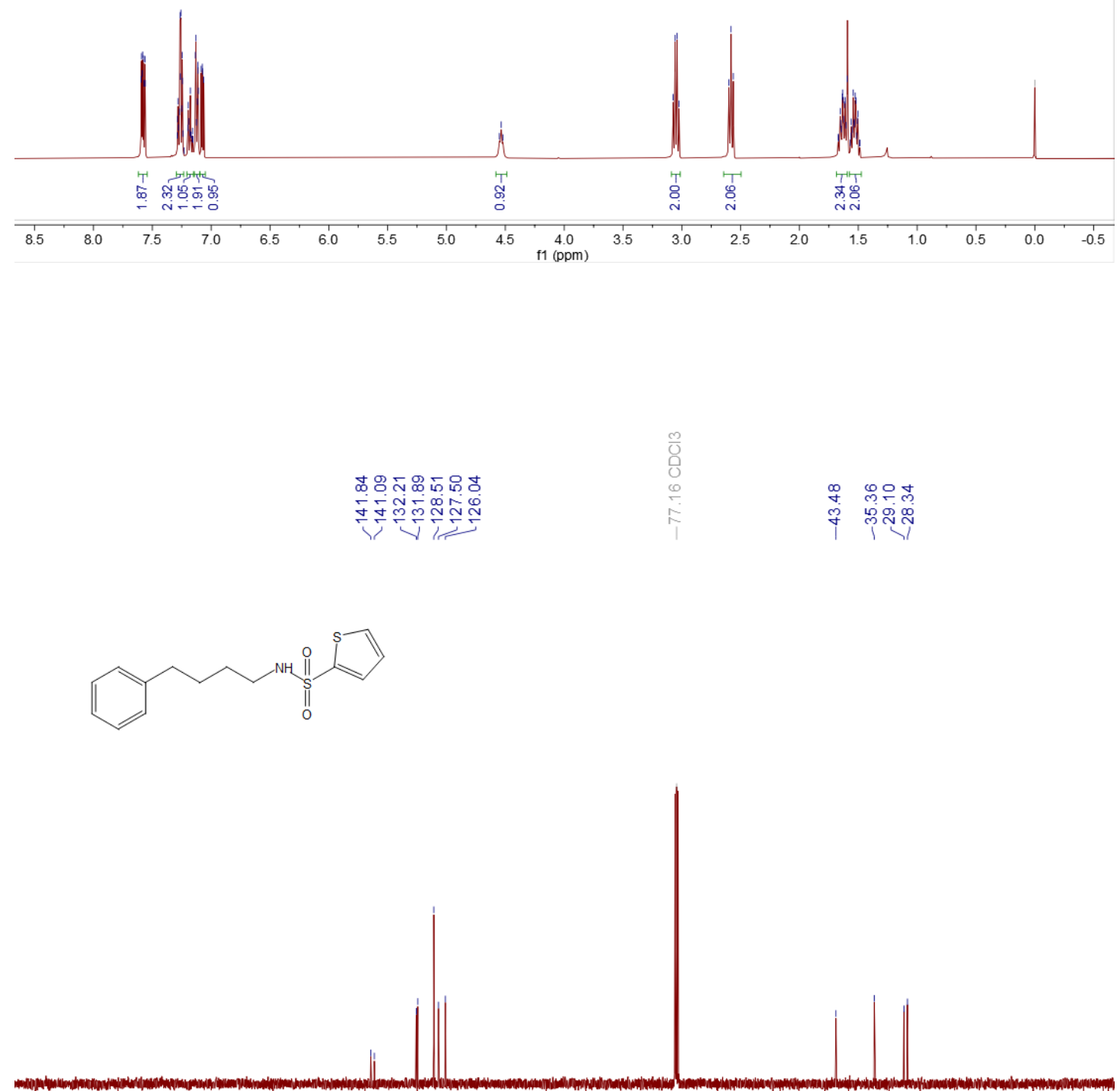

\begin{tabular}{lllllllllllllllllllllll}
\hline 10 & 200 & 190 & 180 & 170 & 160 & 150 & 140 & 130 & 120 & 110 & 100 & 90 & 80 & 70 & 60 & 50 & 40 & 30 & 20 & 10 & 0 & -10 \\
$\mathrm{f} 1(\mathrm{ppm})$ &
\end{tabular} 


\section{Compound 7aa}

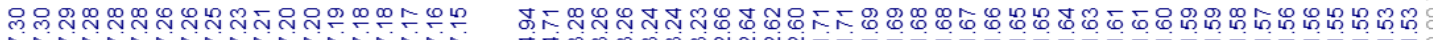

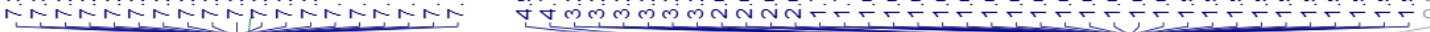

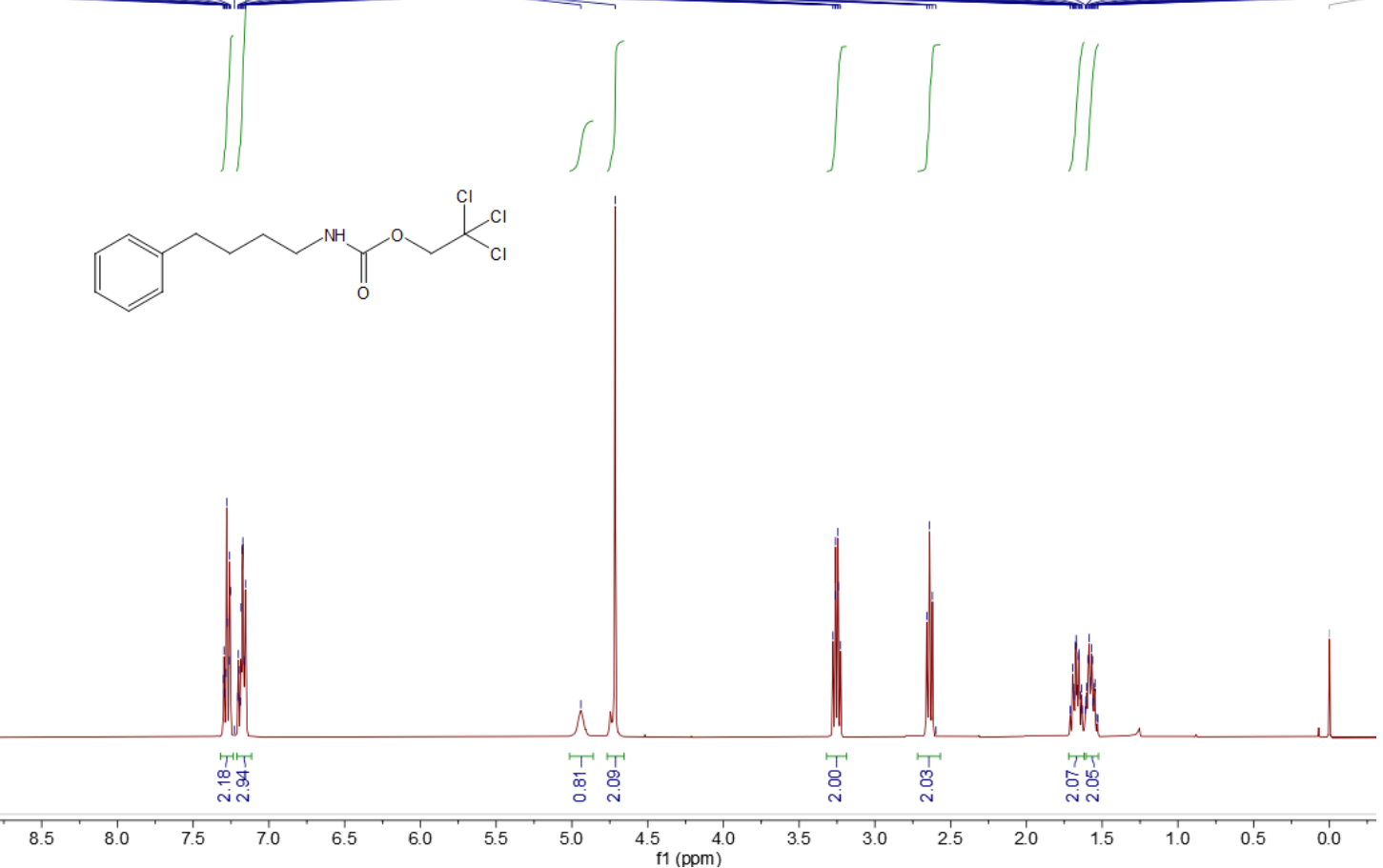

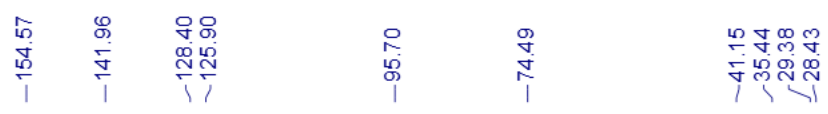<smiles>O=C(NCCCCc1ccccc1)OCC(Cl)(Cl)Cl</smiles>

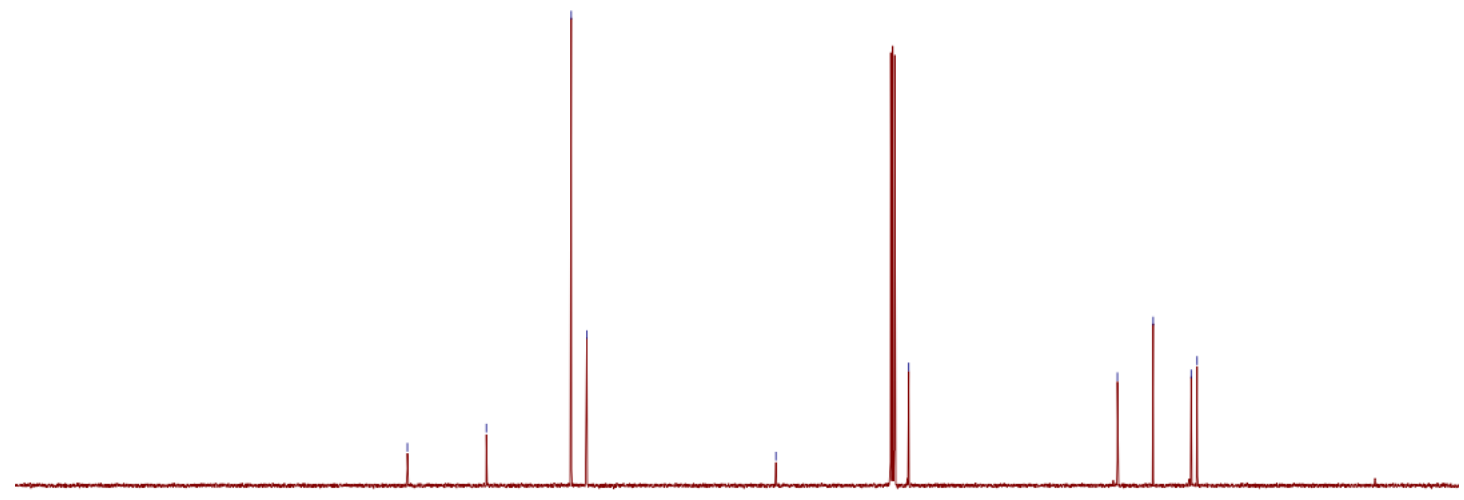

\begin{tabular}{lllllllllllllllllllllllllll}
\hline 1 & 200 & 190 & 180 & 170 & 160 & 150 & 140 & 130 & 120 & 110 & 100 & 90 & 80 & 70 & 60 & 50 & 40 & 30 & 20 & 10 & 0 & -10
\end{tabular} 


\section{Compound 7ba}

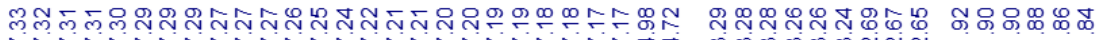

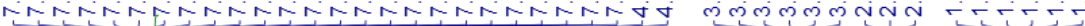

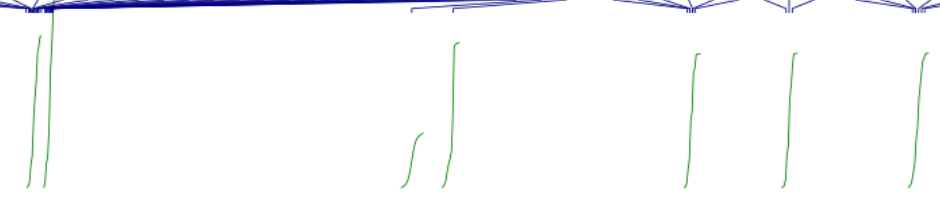

$\curvearrowright>\overbrace{\mathrm{Cl}} \overbrace{\mathrm{NH}}^{\mathrm{O}}$

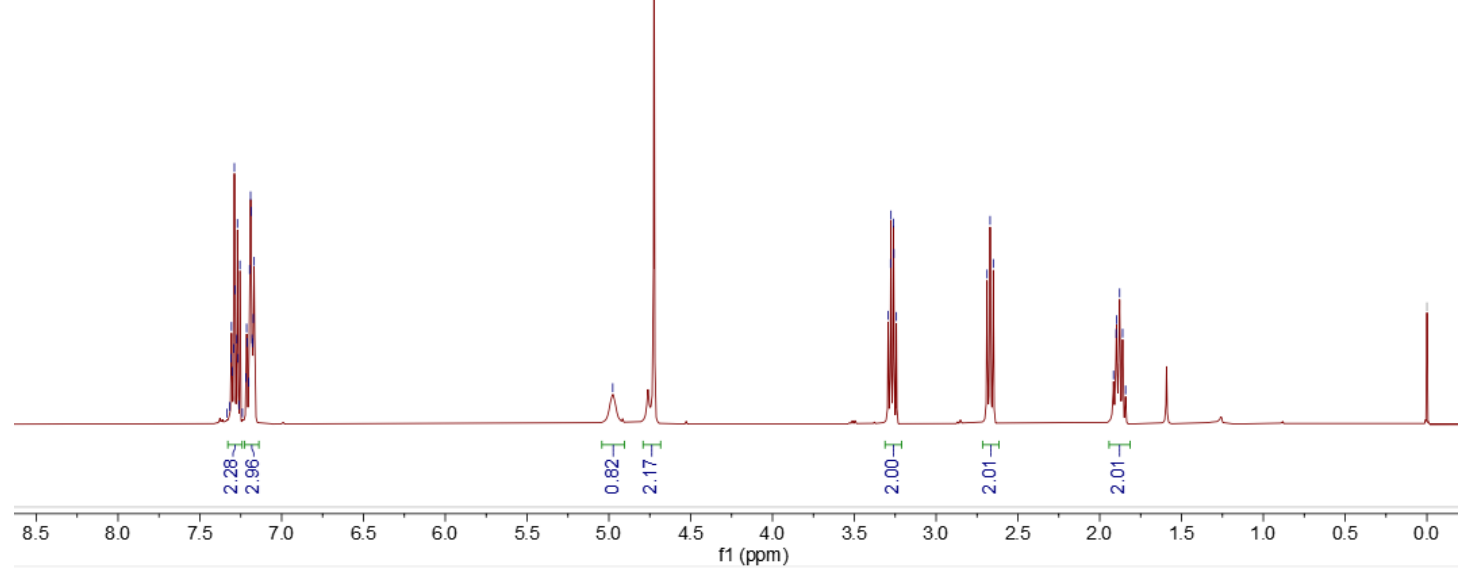

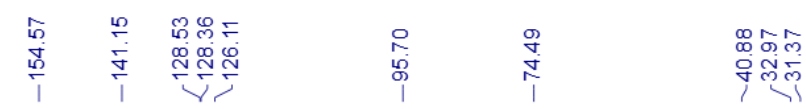<smiles>O=C(NCCCc1ccccc1)OCC(Cl)(Cl)Cl</smiles>

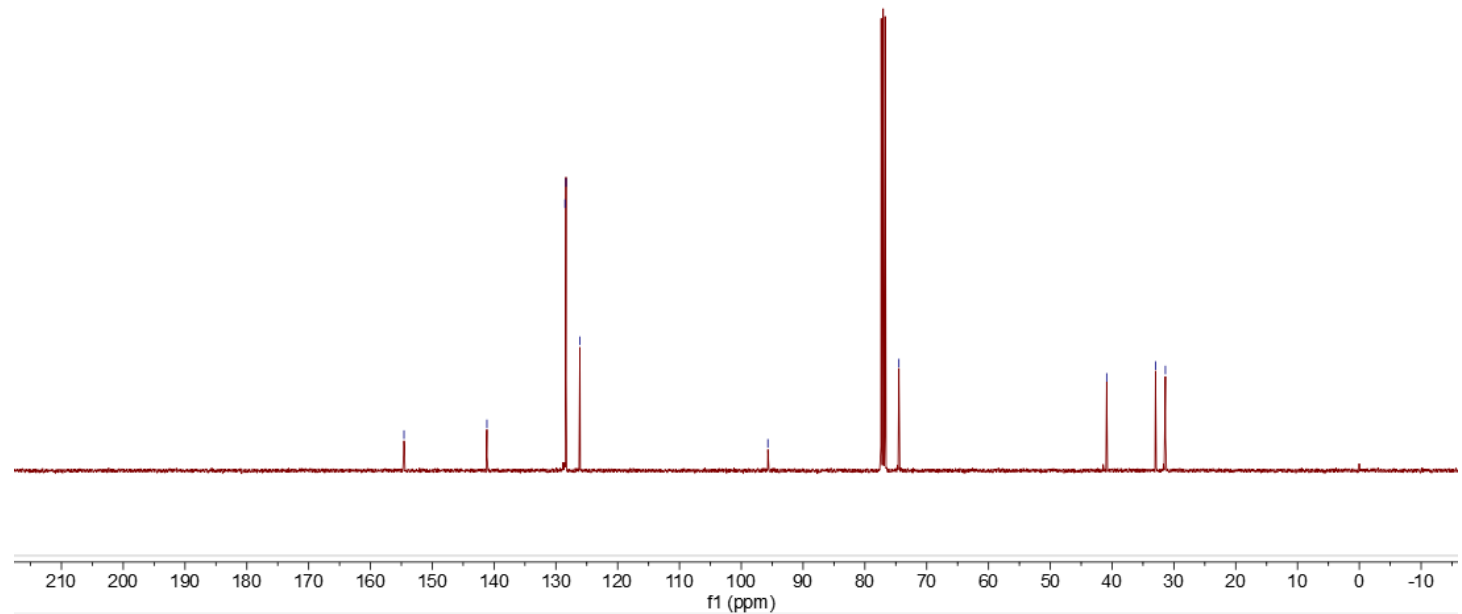




\section{Compound 7ca}

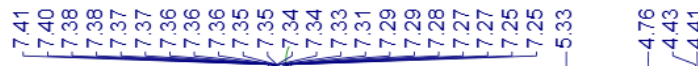<smiles>CC(Cl)(COC(=O)NCc1ccccc1)C1(C)CCCCC1</smiles>

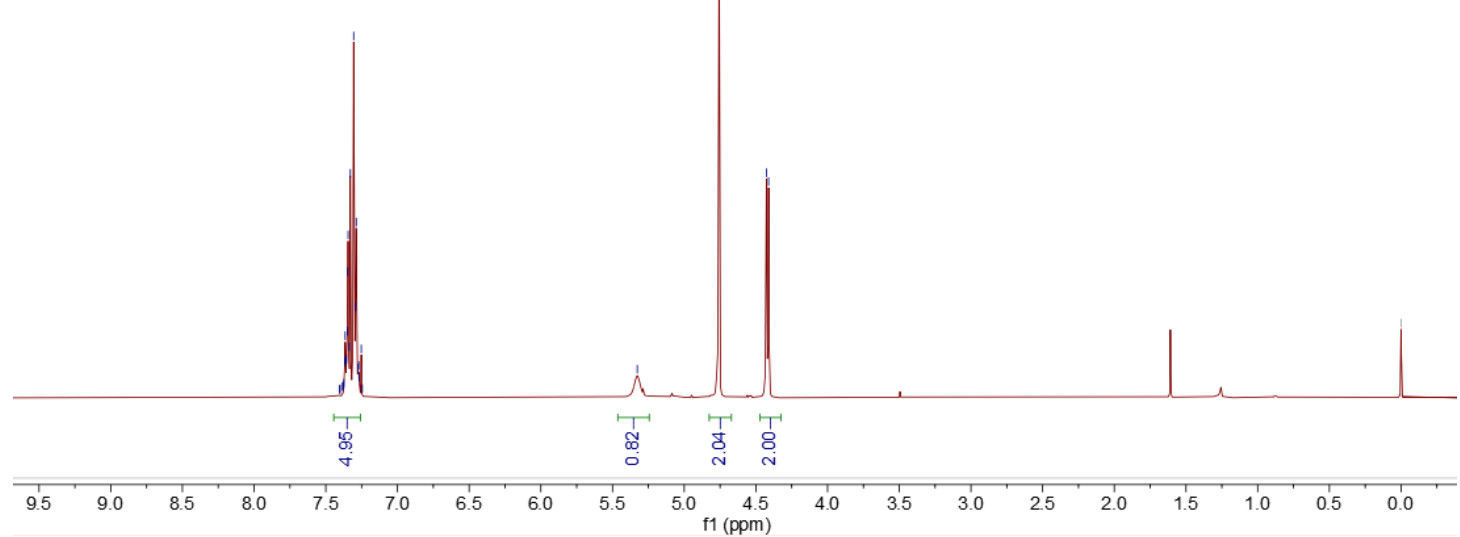

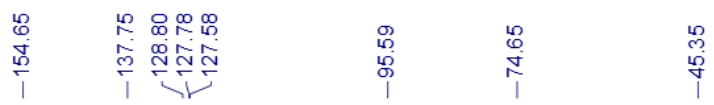
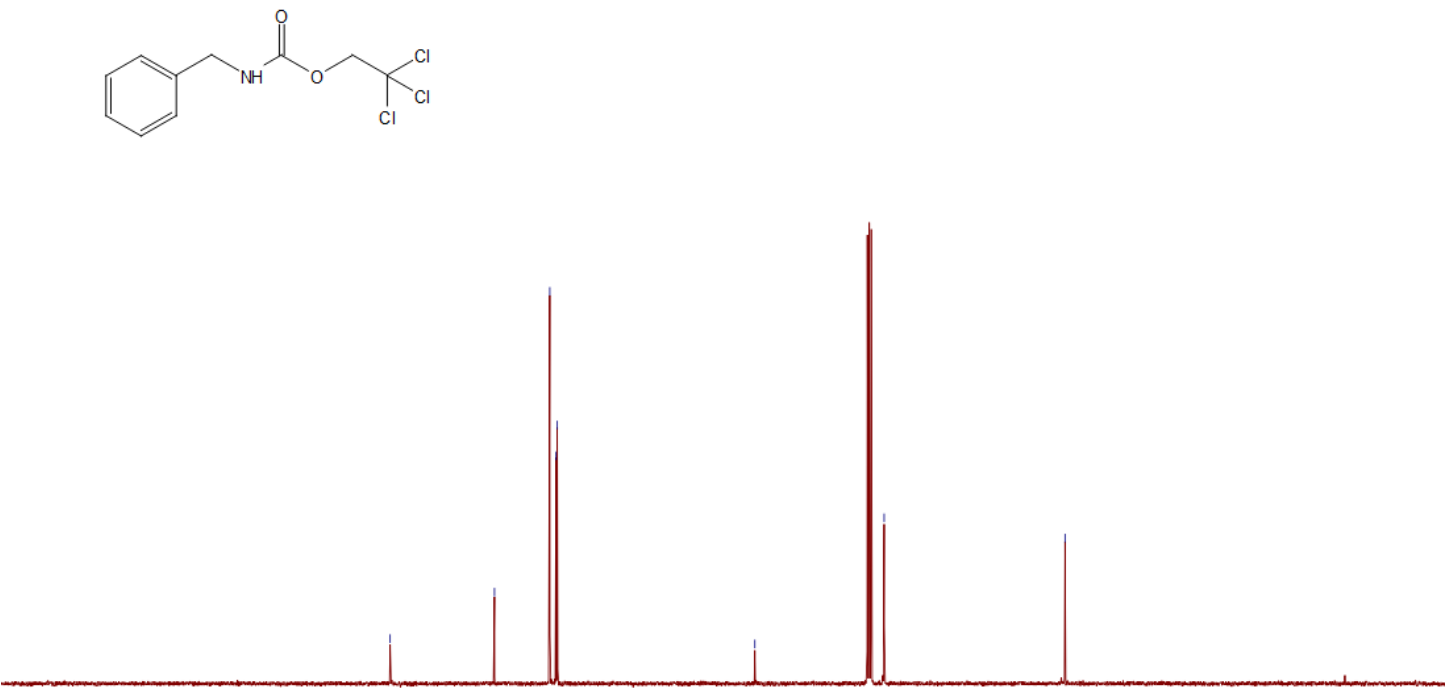

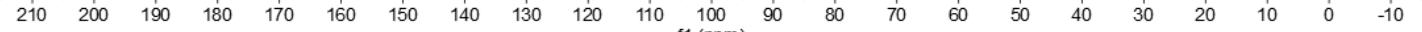


Compound 7da
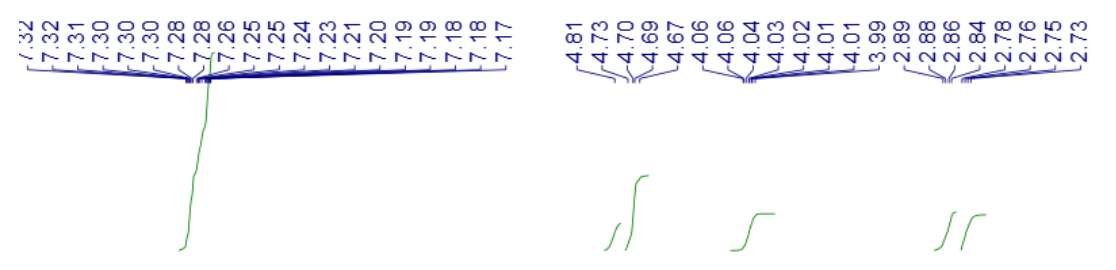

$\stackrel{\infty}{\frac{\infty}{i}}$

$\sum_{k}^{\infty}$
8
0
1
1
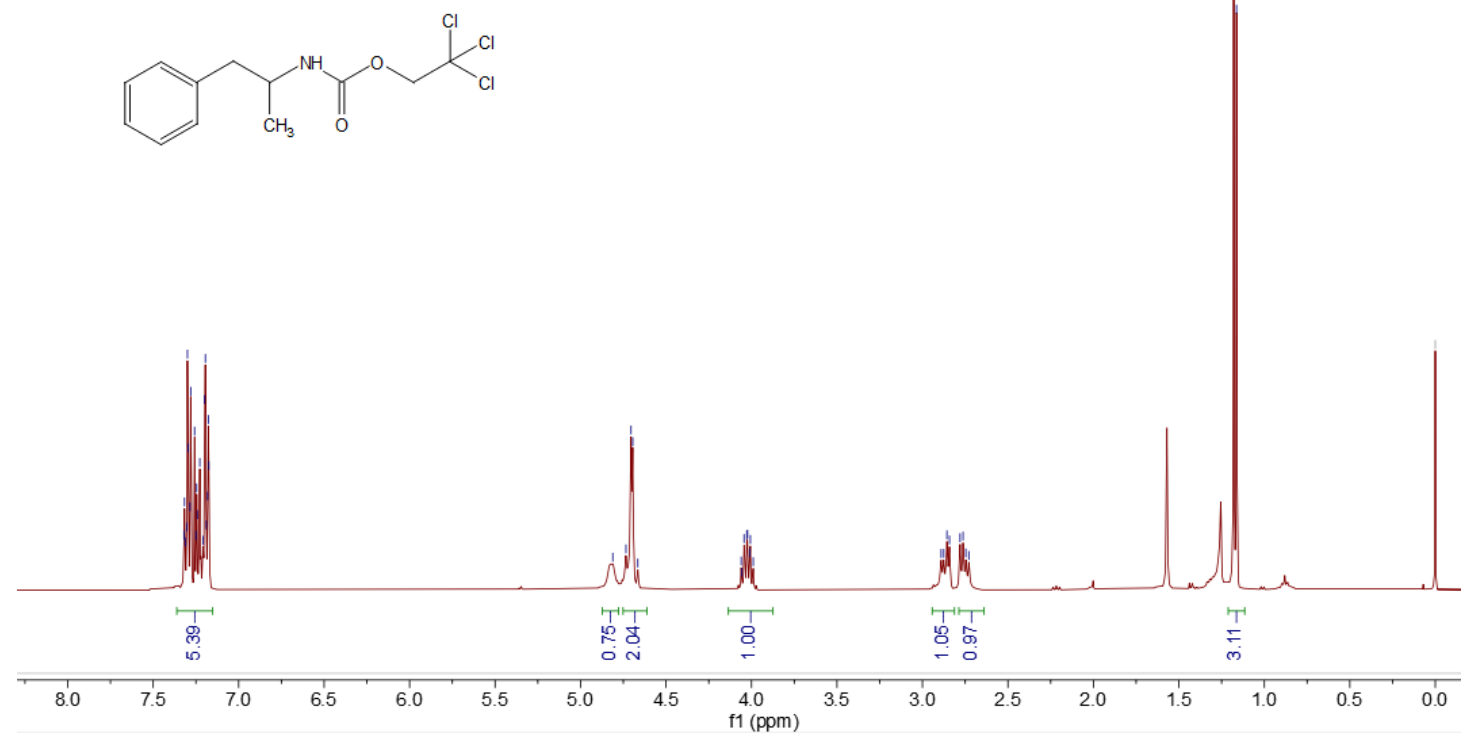

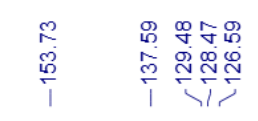
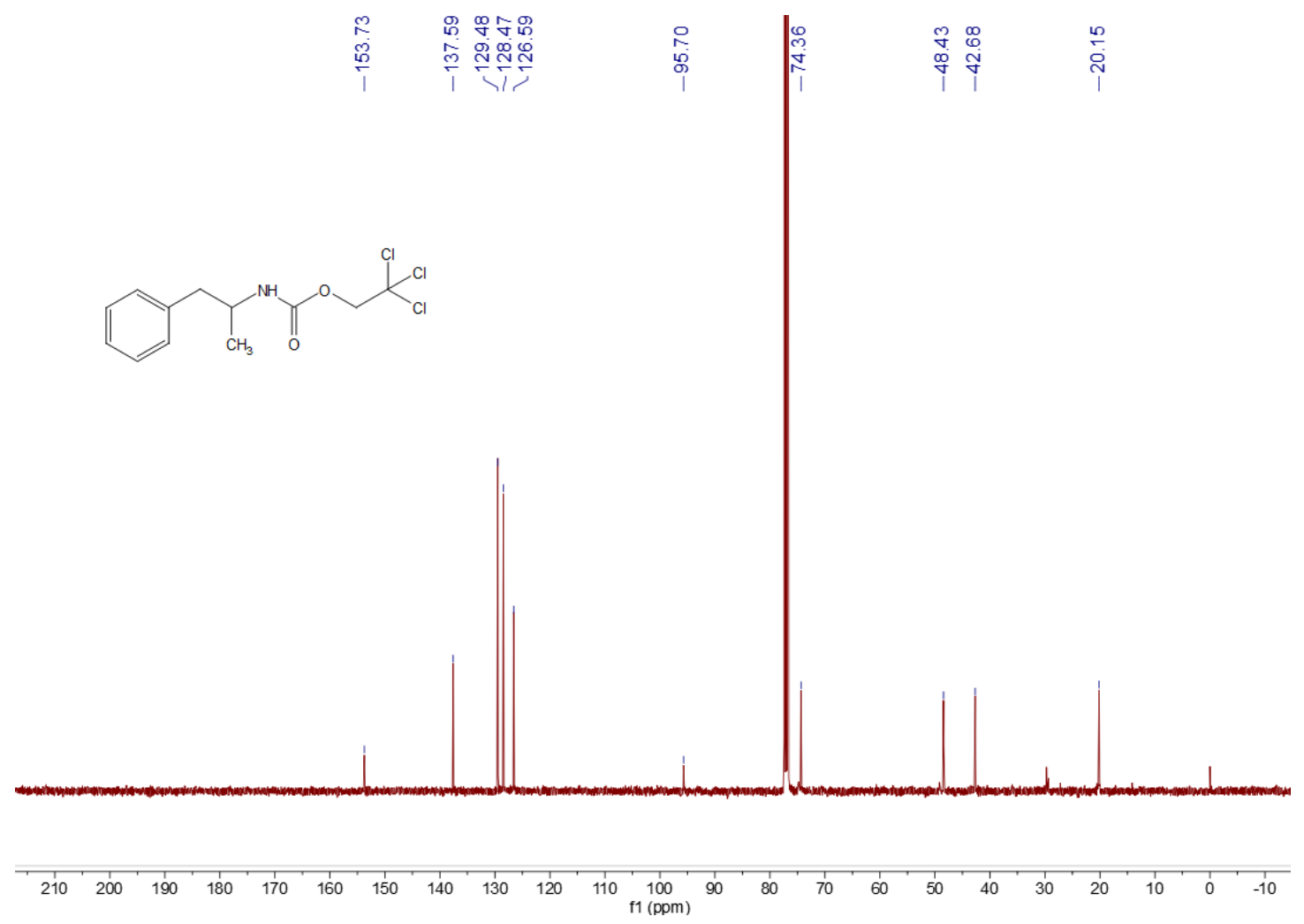

S93 


\section{Compound 7ea}

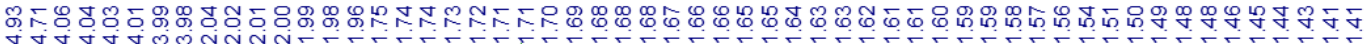<smiles>O=C1CC(O)(Cl)C(CC2CCCC2)C(NC2CCCCC2)CO1</smiles>
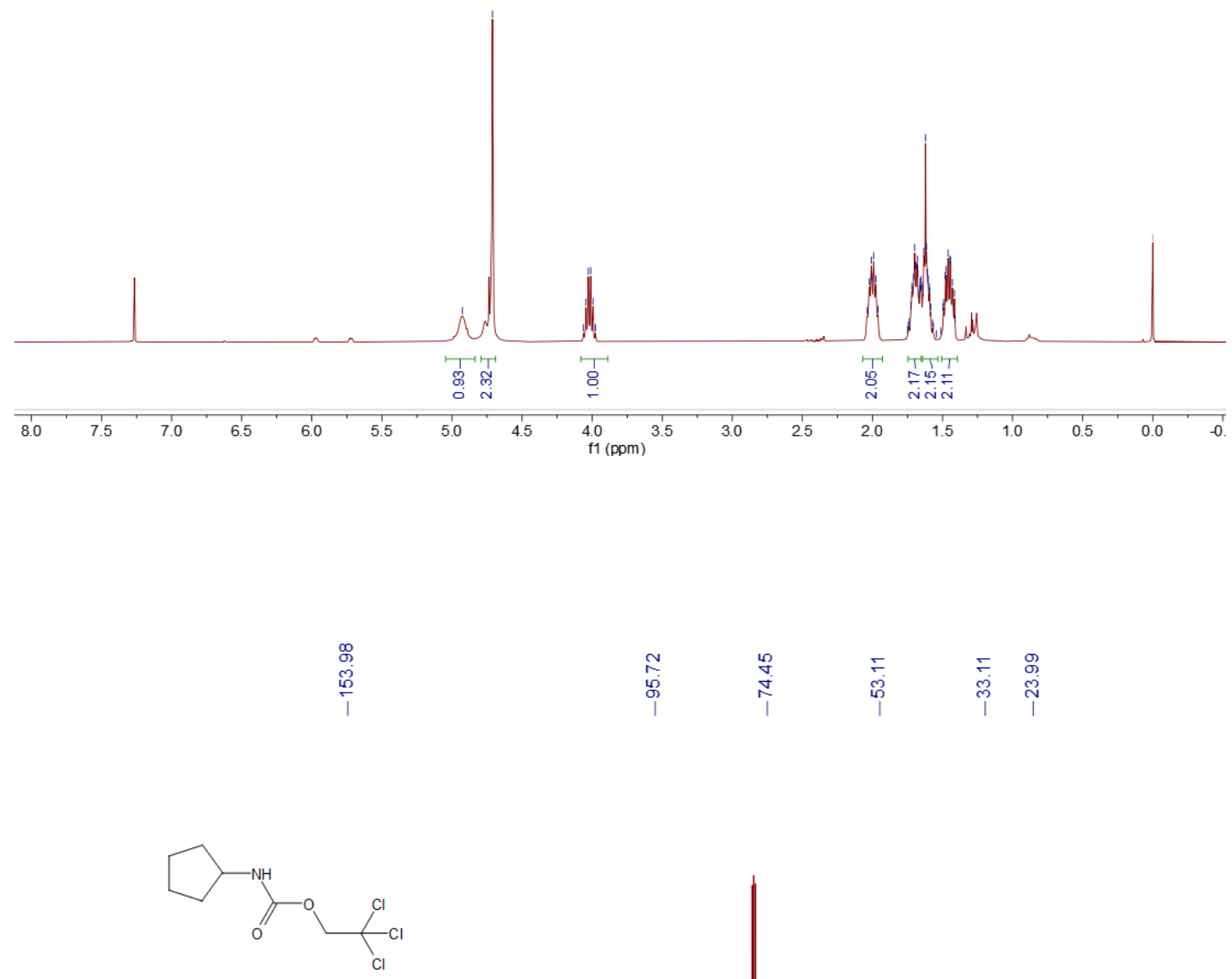


\section{Compound 7fa}
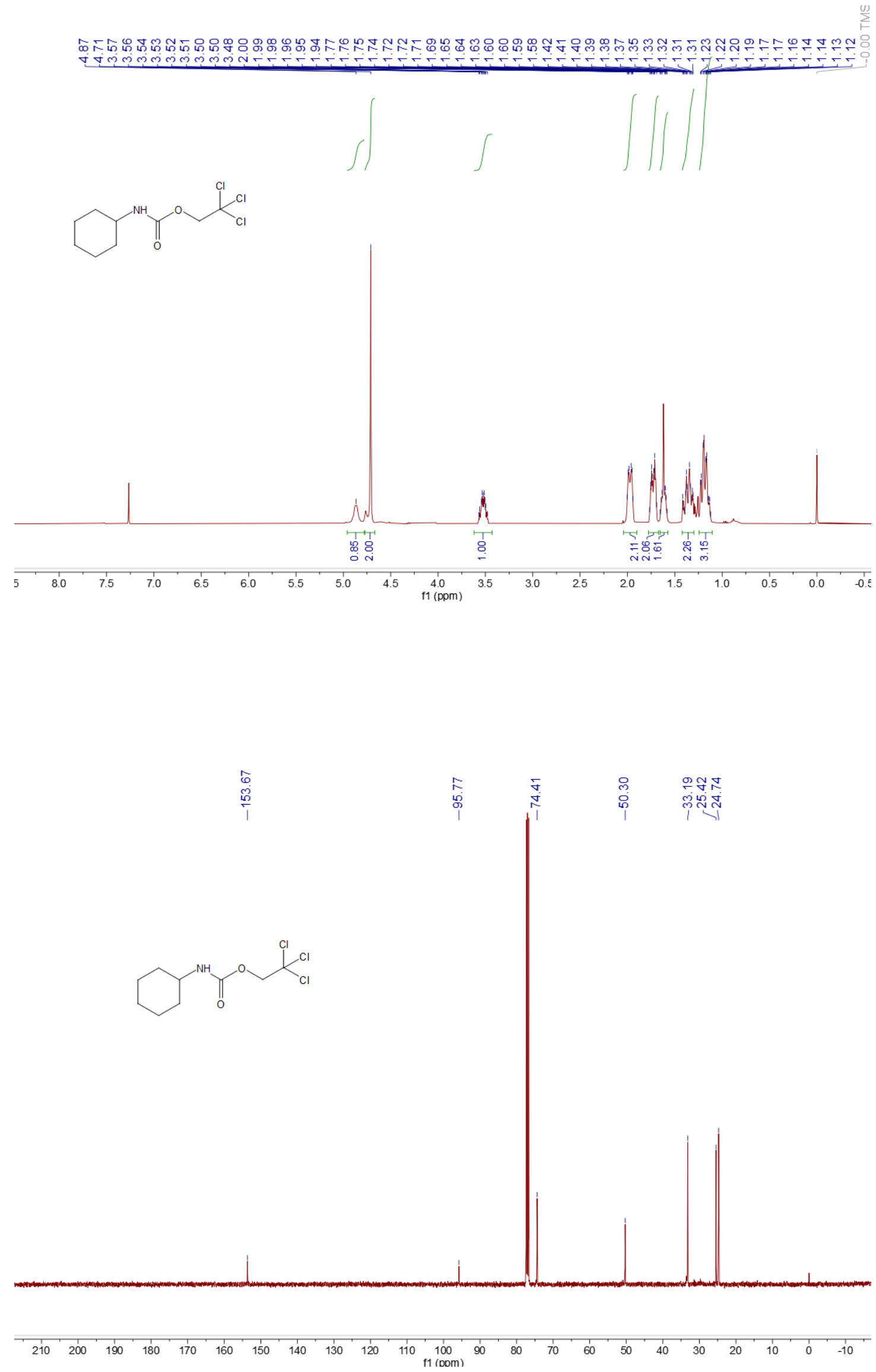
Compound 7ga

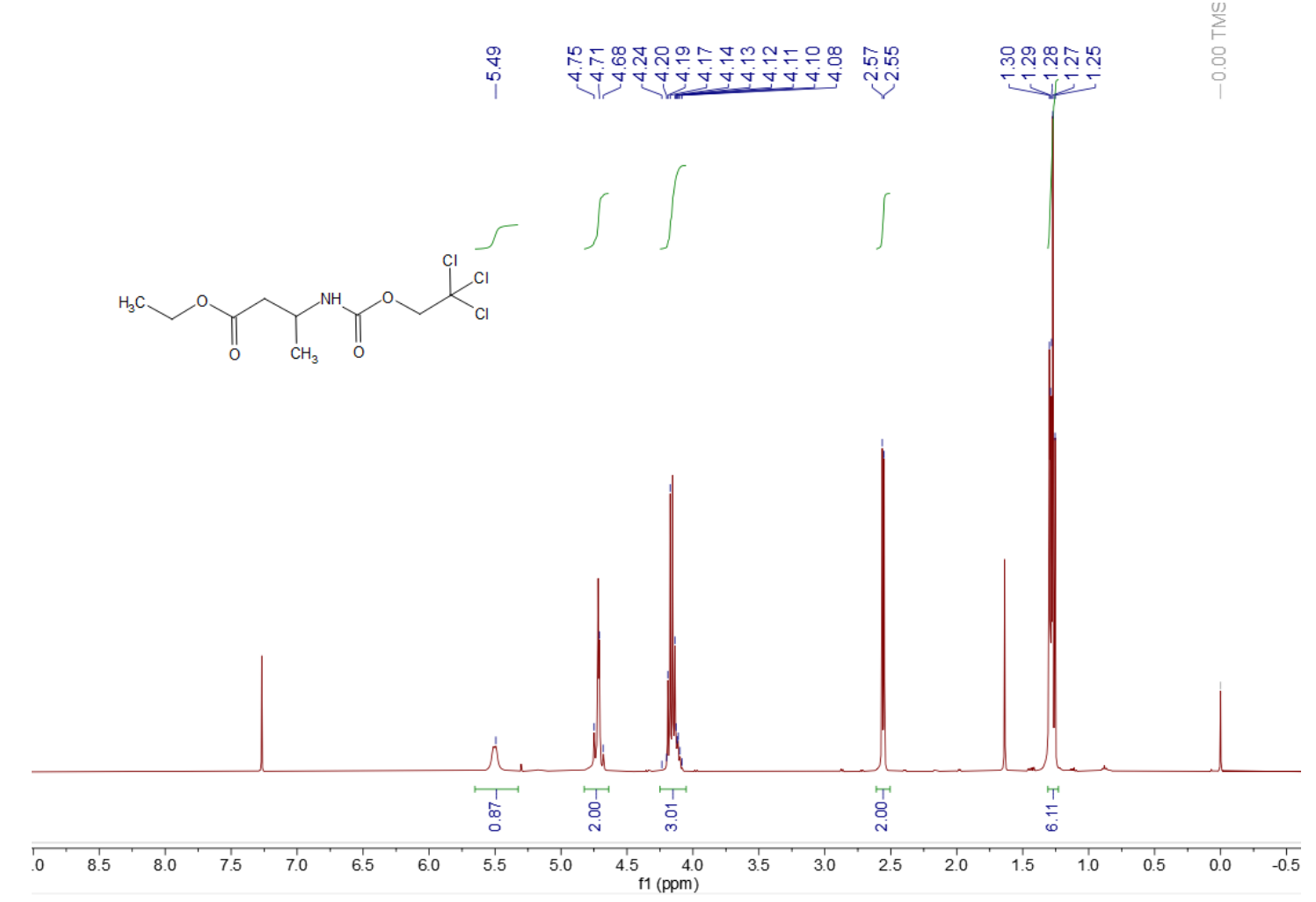

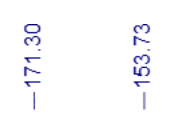

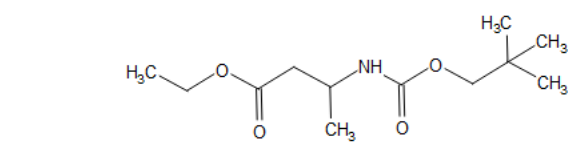

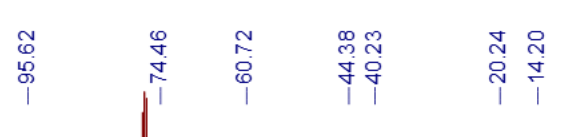
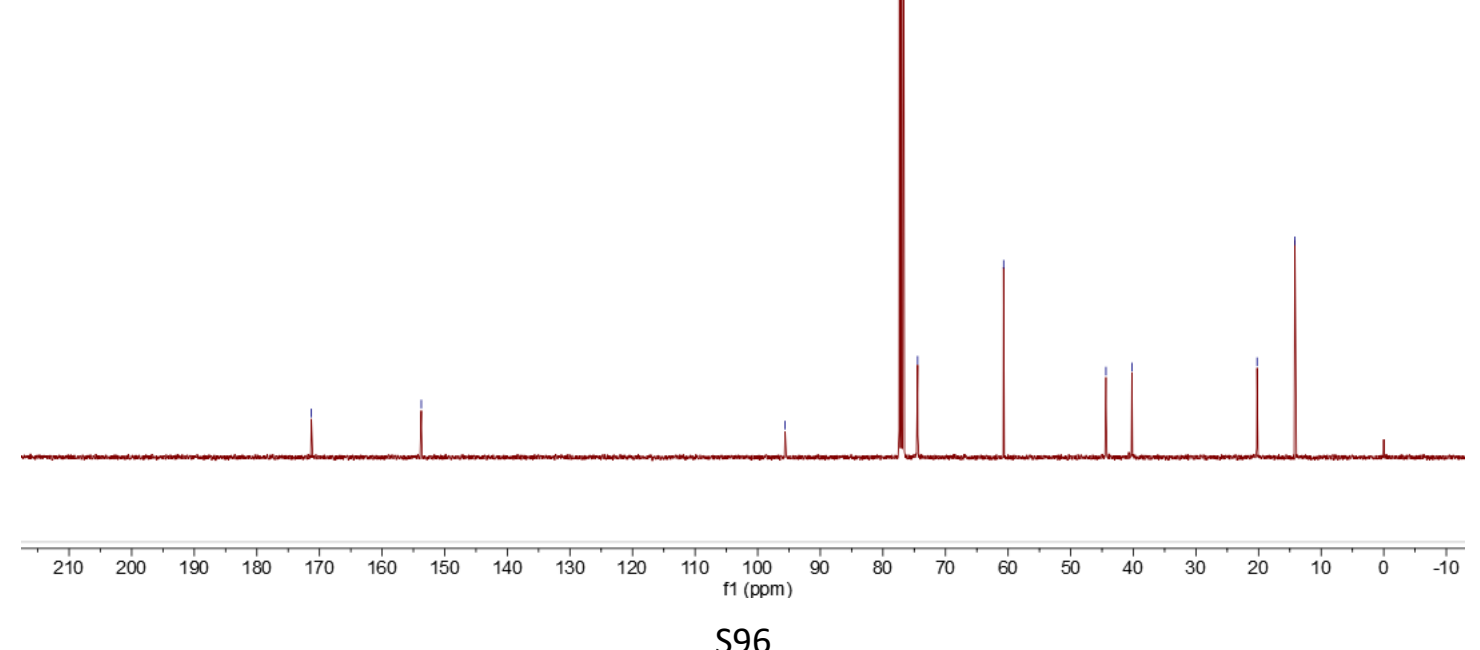


\section{Compound 7ab}

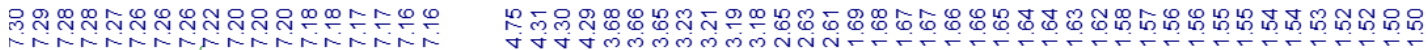

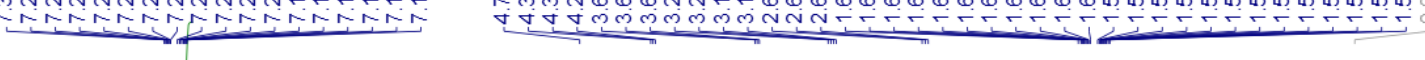

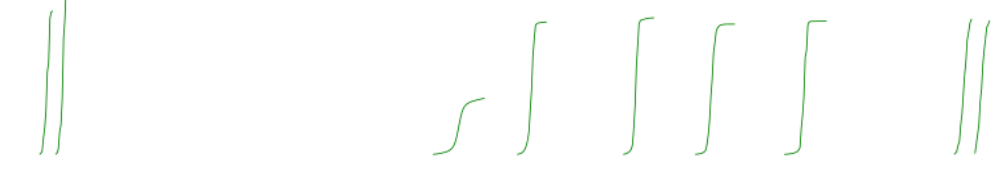<smiles>O=C(NCCCCc1ccccc1)OCCCl</smiles>
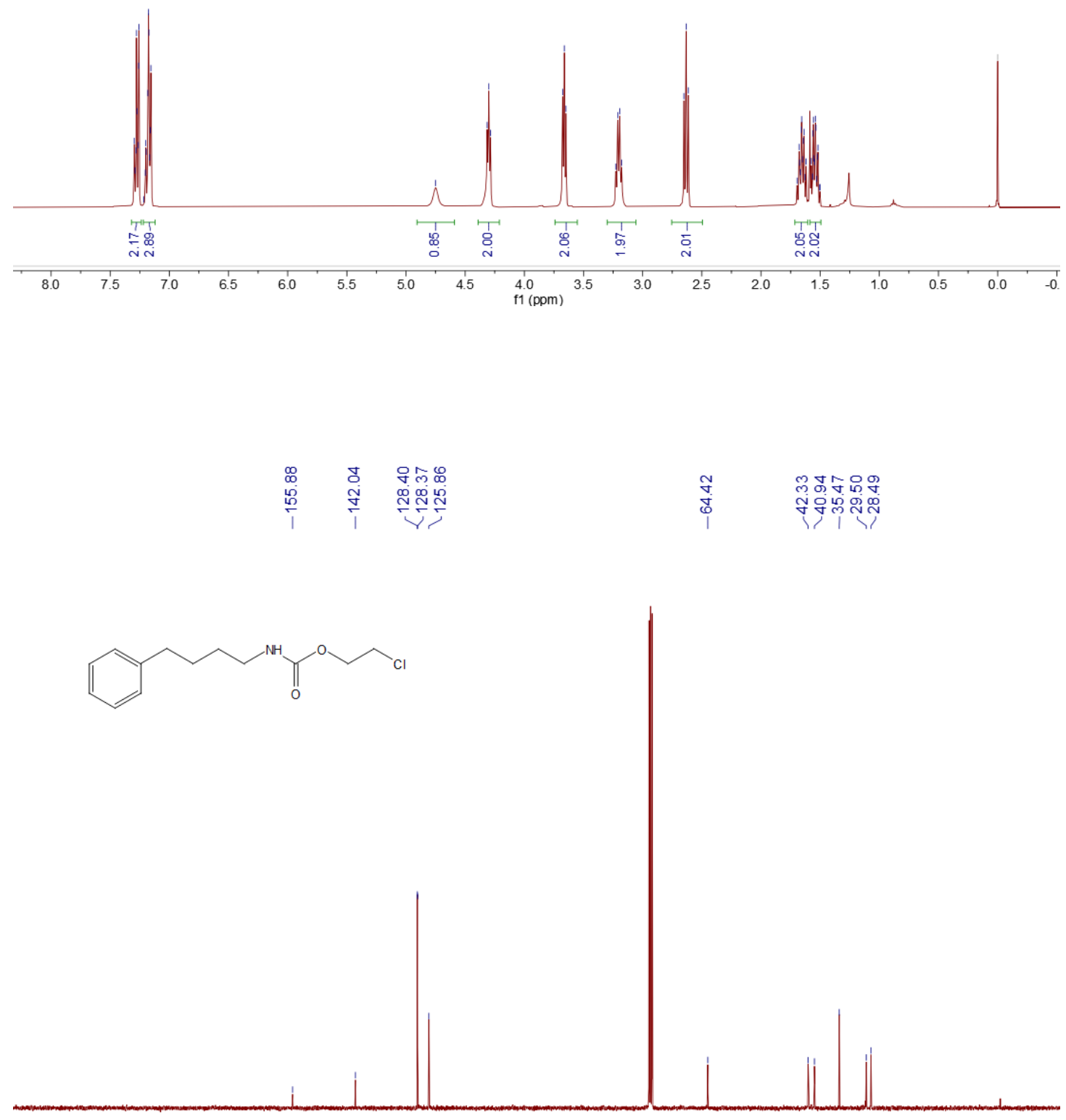

\begin{tabular}{|llllllllllllllllllllllllll}
\hline & 1 & 200 & 190 & 180 & 170 & 160 & 150 & 140 & 130 & 120 & 110 & 100 & 90 & 80 & 70 & 60 & 50 & 40 & 30 & 20 & 10 & 0 & -10 \\
$\mathrm{f} 1(\mathrm{ppm})$
\end{tabular} 


\section{Compound 7ac}

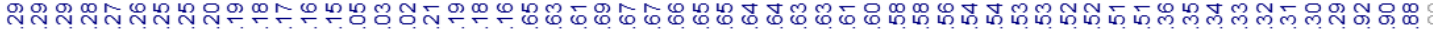<smiles>c1ccccc1</smiles><smiles>CCCCCOC(=O)NCCCCc1ccccc1</smiles>
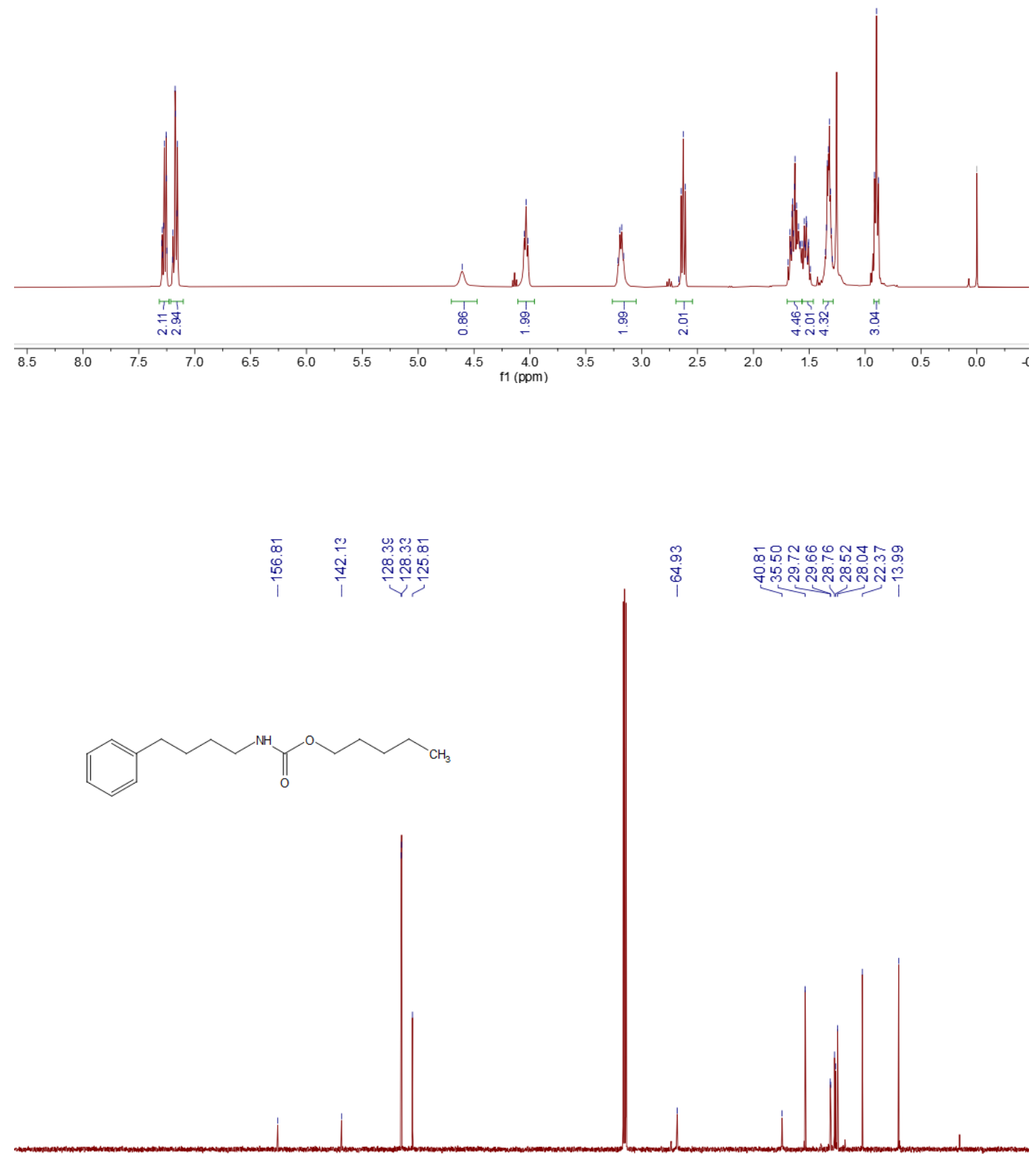

$\begin{array}{llllllllllll}210 & 200 & 190 & 180 & 170 & 160 & 150 & 140 & 130 & 120 & 110 & 100\end{array}$ 


\section{Compound 7ad}

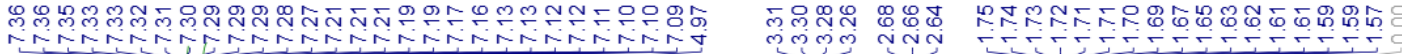
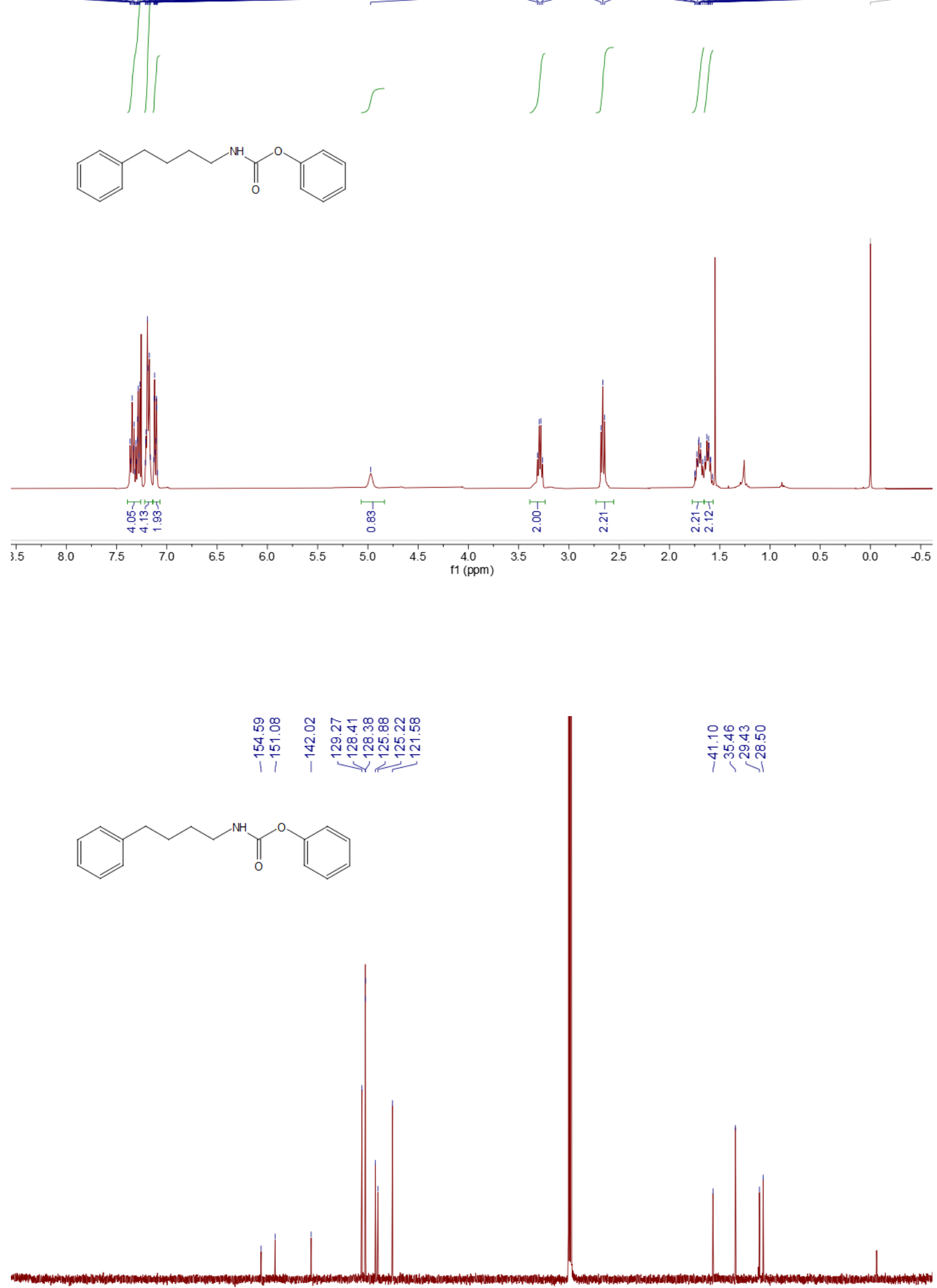

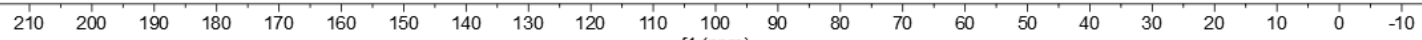




\section{Compound 7be}

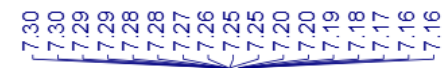

$\stackrel{\substack{0 \\ \hdashline}}{i}$

लm

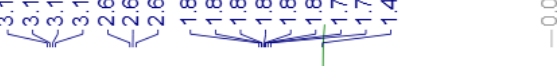

$\curvearrowright \overbrace{\mathrm{NH}}^{\mathrm{O}} \mathrm{CH}_{\mathrm{CH}_{3}}^{\mathrm{CH}_{3}}$
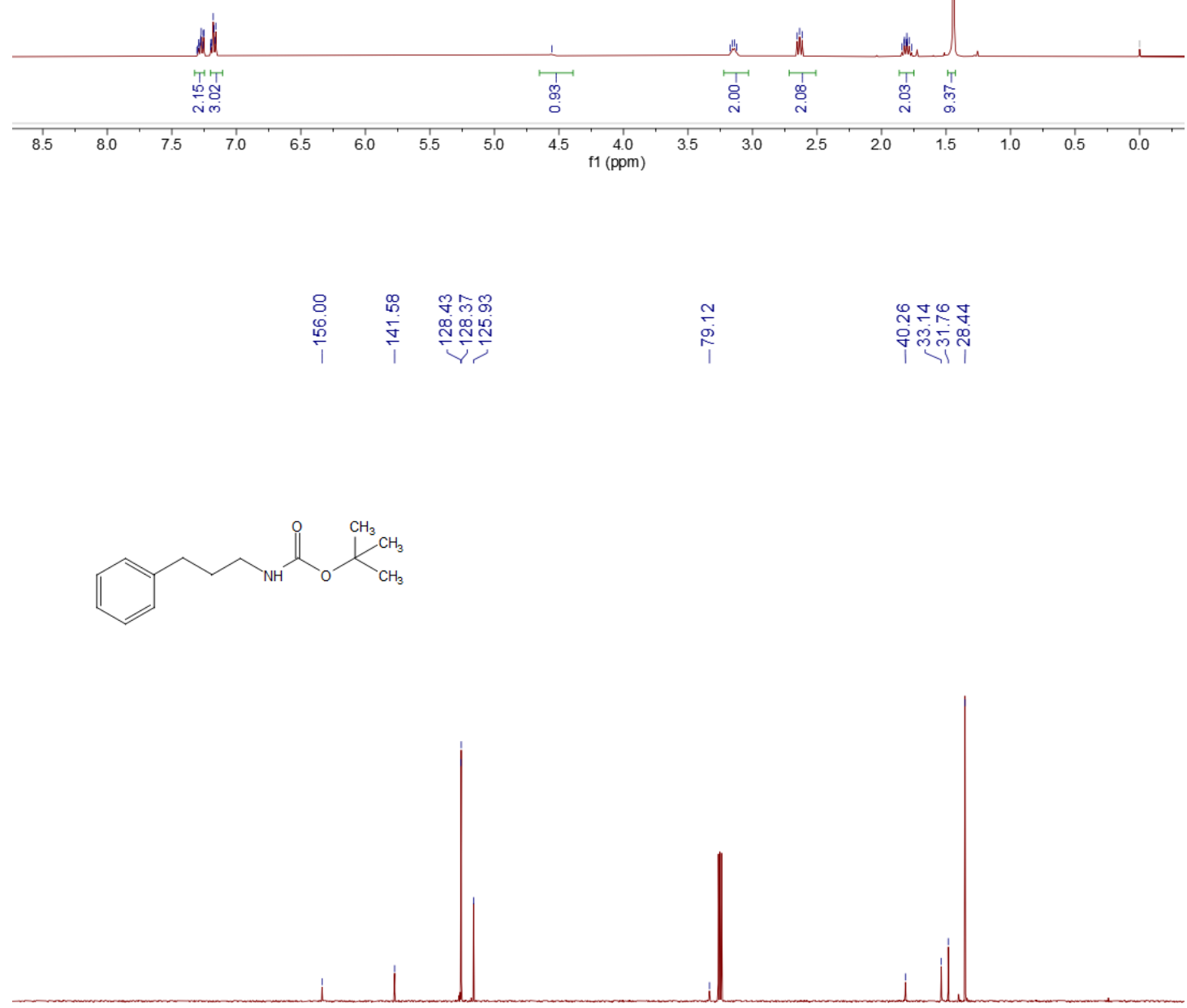

$\begin{array}{lllllllllllllllllllllllllll}1 & 1 & 200 & 190 & 180 & 170 & 160 & 150 & 140 & 130 & 120 & 110 & 100 & 90 & 80 & 70 & 60 & 50 & 40 & 30 & 20 & 10 & 0 & -10\end{array}$ 


\section{Cartesian Coordinates and Energies}

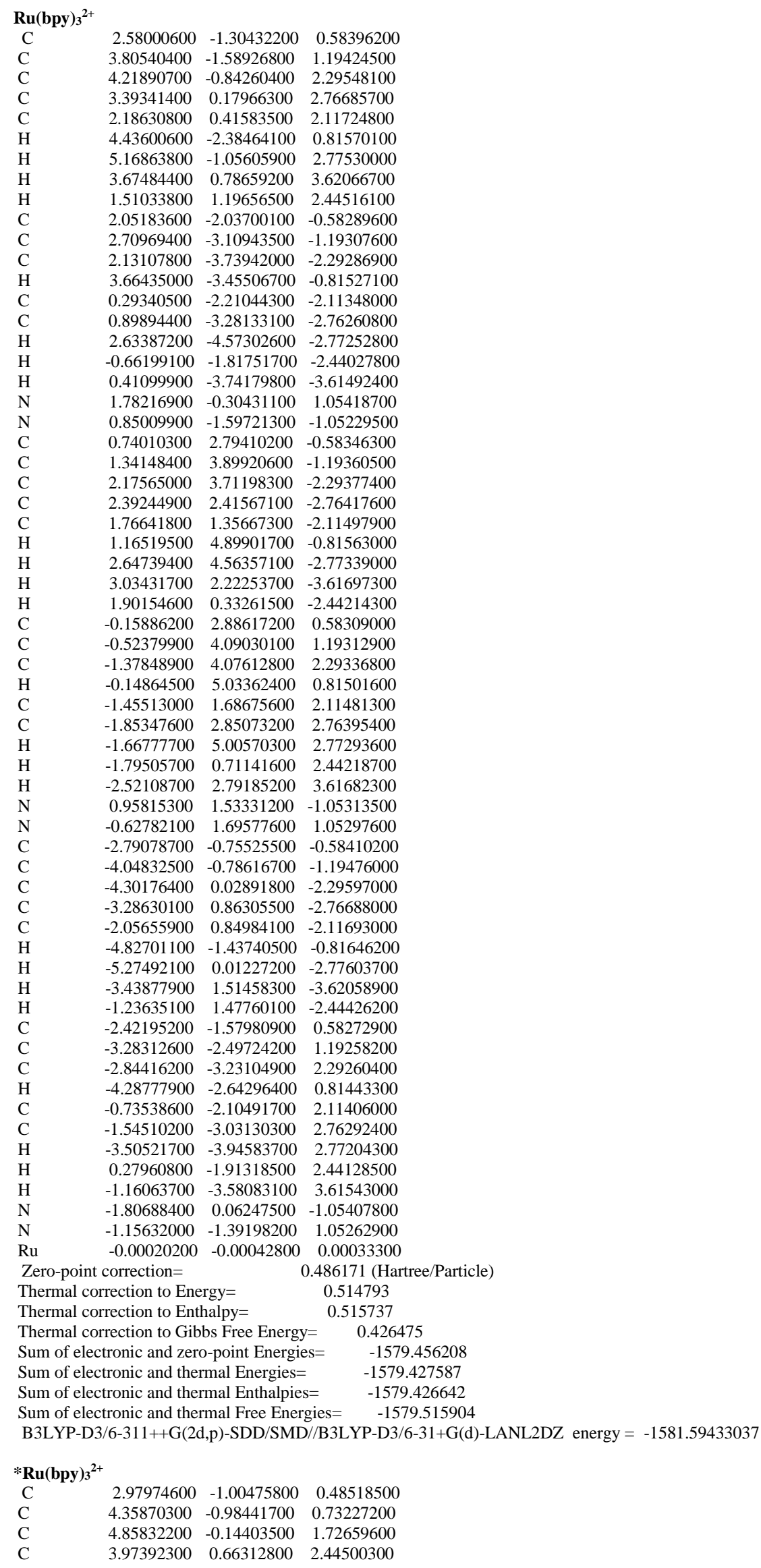




\begin{tabular}{|c|c|c|c|}
\hline $\mathrm{C}$ & 2.61828100 & 0.59934100 & 2.12787200 \\
\hline $\mathrm{H}$ & 5.03889600 & -1.59730800 & 0.15236400 \\
\hline $\mathrm{H}$ & 5.92402000 & -0.11595100 & 1.93127600 \\
\hline $\mathrm{H}$ & 4.32322000 & 1.32745500 & 3.22836600 \\
\hline $\mathrm{H}$ & 1.88777300 & 1.21371800 & 2.64794900 \\
\hline $\mathrm{C}$ & 2.36356400 & -1.86908300 & -0.55201700 \\
\hline $\mathrm{C}$ & 3.03136300 & -2.96633400 & -1.10859900 \\
\hline $\mathrm{C}$ & 2.40826500 & -3.73628200 & -2.08781500 \\
\hline $\mathrm{H}$ & 4.02409700 & -3.23088700 & -0.76486100 \\
\hline $\mathrm{C}$ & 0.49683400 & -2.30958000 & -1.89020500 \\
\hline $\mathrm{C}$ & 1.11560500 & -3.39885300 & -2.49217700 \\
\hline $\mathrm{H}$ & 2.92113700 & -4.58931200 & -2.52081600 \\
\hline $\mathrm{H}$ & -0.51084500 & -2.00887300 & -2.15559100 \\
\hline $\mathrm{H}$ & 0.59072300 & -3.96823100 & -3.25158800 \\
\hline $\mathrm{N}$ & 2.13379500 & -0.21455300 & 1.18090400 \\
\hline $\mathrm{N}$ & 1.10017500 & -1.55880100 & -0.94782000 \\
\hline $\mathrm{C}$ & 0.52111400 & 2.90052700 & -0.51857700 \\
\hline $\mathrm{C}$ & 1.07165100 & 4.05852900 & -1.07596800 \\
\hline $\mathrm{C}$ & 2.05652300 & 3.95160600 & -2.05512700 \\
\hline $\mathrm{C}$ & 2.47709000 & 2.68268300 & -2.45939400 \\
\hline $\mathrm{C}$ & 1.89646200 & 1.56803700 & -1.86615600 \\
\hline $\mathrm{H}$ & 0.73640400 & 5.03673500 & -0.75303500 \\
\hline $\mathrm{H}$ & 2.48805400 & 4.84500800 & -2.49502400 \\
\hline $\mathrm{H}$ & 3.24041300 & 2.55467000 & -3.21921900 \\
\hline $\mathrm{H}$ & 2.18946500 & 0.56179800 & -2.14164600 \\
\hline $\mathrm{C}$ & -0.52218800 & 2.90032500 & 0.51865600 \\
\hline $\mathrm{C}$ & -1.07337000 & 4.05818100 & 1.07574800 \\
\hline $\mathrm{C}$ & -2.05811600 & 3.95097300 & 2.05499700 \\
\hline $\mathrm{H}$ & -0.73872600 & 5.03650200 & 0.75253800 \\
\hline $\mathrm{C}$ & -1.89667300 & 1.56746000 & 1.86677100 \\
\hline $\mathrm{C}$ & -2.47792500 & 2.68195100 & 2.45971400 \\
\hline $\mathrm{H}$ & -2.49013800 & 4.84426300 & 2.49464200 \\
\hline $\mathrm{H}$ & -2.18904500 & 0.56112900 & 2.14259100 \\
\hline $\mathrm{H}$ & -3.24110400 & 2.55376500 & 3.21965200 \\
\hline $\mathrm{N}$ & 0.94130600 & 1.66645200 & -0.92124200 \\
\hline $\mathrm{N}$ & -0.94167700 & 1.66614700 & 0.92176000 \\
\hline $\mathrm{C}$ & -2.97929400 & -1.00545300 & -0.48539400 \\
\hline $\mathrm{C}$ & -4.35822800 & -0.98530200 & -0.73263600 \\
\hline $\mathrm{C}$ & -4.85784100 & -0.14508700 & -1.72710200 \\
\hline $\mathrm{C}$ & -3.97346100 & 0.66210800 & -2.44549600 \\
\hline $\mathrm{C}$ & -2.61784700 & 0.59852500 & -2.12820100 \\
\hline $\mathrm{H}$ & -5.03841500 & -1.59820700 & -0.15273800 \\
\hline $\mathrm{H}$ & -5.92352000 & -0.11715500 & -1.93190200 \\
\hline $\mathrm{H}$ & -4.32274900 & 1.32630600 & -3.22897200 \\
\hline $\mathrm{H}$ & -1.88736000 & 1.21293900 & -2.64826300 \\
\hline $\mathrm{C}$ & -2.36310700 & -1.86958100 & 0.55197200 \\
\hline $\mathrm{C}$ & -3.03080900 & -2.96689100 & 1.10856500 \\
\hline $\mathrm{C}$ & -2.40769400 & -3.73668400 & 2.08789200 \\
\hline $\mathrm{H}$ & -4.02346700 & -3.23161700 & 0.76475100 \\
\hline $\mathrm{C}$ & -0.49644900 & -2.30972300 & 1.89036900 \\
\hline $\mathrm{C}$ & -1.11511700 & -3.39905000 & 2.49234600 \\
\hline $\mathrm{H}$ & -2.92048200 & -4.58976200 & 2.52090000 \\
\hline $\mathrm{H}$ & 0.51115900 & -2.00885100 & 2.15584100 \\
\hline $\mathrm{H}$ & -0.59022100 & -3.96831100 & 3.25183500 \\
\hline $\mathrm{N}$ & -2.13336200 & -0.21520600 & -1.18109200 \\
\hline $\mathrm{N}$ & -1.09980700 & -1.55908000 & 0.94788800 \\
\hline $\mathrm{Ru}$ & 0.00003900 & 0.03140700 & 0.00008200 \\
\hline \multicolumn{2}{|c|}{ Zero-point correction $=$} & & 0.483664 (Hartree/Particle) \\
\hline \multicolumn{3}{|c|}{ Thermal correction to Energy= } & 0.513579 \\
\hline \multicolumn{3}{|c|}{ Thermal correction to Enthalpy= } & 0.514524 \\
\hline \multicolumn{3}{|c|}{ Thermal correction to Gibbs Free Energy= } & 0.419332 \\
\hline \multicolumn{3}{|c|}{ Sum of electronic and zero-point Energies } & -1579.384550 \\
\hline \multicolumn{3}{|c|}{ Sum of electronic and thermal Energies= } & -1579.354634 \\
\hline \multicolumn{3}{|c|}{ Sum of electronic and thermal Enthalpies= } & -1579.353690 \\
\hline \multicolumn{4}{|c|}{ Sum of electronic and thermal Free Energies= } \\
\hline
\end{tabular}

Sum of electronic and thermal Free Energies $=-1579.448882$

(U)B3LYP-D3/6-311++G(2d,p)-SDD/SMD//(U)B3LYP-D3/6-31+G(d)-LANL2DZ energy = -1581.519862

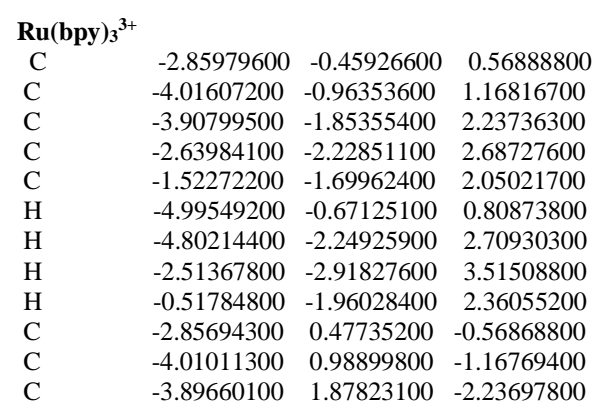




\begin{tabular}{|c|c|c|c|}
\hline $\mathrm{H}$ & -4.99129800 & 0.70301100 & -0.80801000 \\
\hline $\mathrm{C}$ & -1.51232300 & 1.70894800 & -2.05052300 \\
\hline $\mathrm{C}$ & -2.62619200 & 2.24493900 & -2.68733700 \\
\hline $\mathrm{H}$ & -4.78832100 & 2.27963800 & -2.70869700 \\
\hline $\mathrm{H}$ & -0.50583900 & 1.96289800 & -2.36120300 \\
\hline $\mathrm{H}$ & -2.49583600 & 2.93375300 & -3.51529000 \\
\hline $\mathrm{N}$ & -1.62745900 & -0.83204700 & 1.02398000 \\
\hline $\mathrm{N}$ & -1.62236500 & 0.84224000 & -1.02412400 \\
\hline $\mathrm{C}$ & 1.01484500 & -2.71293700 & -0.56835600 \\
\hline $\mathrm{C}$ & 1.14824500 & -3.96751600 & -1.16721300 \\
\hline $\mathrm{C}$ & 0.32122200 & -4.31400000 & -2.23630900 \\
\hline $\mathrm{C}$ & -0.63167400 & -3.39723100 & -2.68658700 \\
\hline $\mathrm{C}$ & -0.72431400 & -2.16449600 & -2.04995500 \\
\hline $\mathrm{H}$ & 1.88657700 & -4.67419700 & -0.80755800 \\
\hline $\mathrm{H}$ & 0.41938300 & -5.28701900 & -2.70791300 \\
\hline $\mathrm{H}$ & -1.29355900 & -3.62887000 & -3.51436500 \\
\hline $\mathrm{H}$ & -1.44767600 & -1.42003200 & -2.36069100 \\
\hline $\mathrm{C}$ & 1.82761400 & -2.24695600 & 0.56900000 \\
\hline $\mathrm{C}$ & 2.84258900 & -2.99608500 & 1.16818500 \\
\hline $\mathrm{C}$ & 3.55970500 & -2.45719700 & 2.23698200 \\
\hline $\mathrm{H}$ & 3.07902500 & -3.99053500 & 0.80894600 \\
\hline $\mathrm{C}$ & 2.23380400 & -0.46845100 & 2.04971500 \\
\hline $\mathrm{C}$ & 3.25059100 & -1.17131300 & 2.68662500 \\
\hline $\mathrm{H}$ & 4.34960200 & -3.03360500 & 2.70881700 \\
\hline $\mathrm{H}$ & 1.95716200 & 0.53222200 & 2.35976600 \\
\hline $\mathrm{H}$ & 3.78519200 & -0.71695600 & 3.51410700 \\
\hline $\mathrm{N}$ & 0.08150100 & -1.82623800 & -1.02375000 \\
\hline $\mathrm{N}$ & 1.53445600 & -0.99322500 & 1.02389600 \\
\hline $\mathrm{C}$ & 1.84215800 & 2.23529800 & -0.56858300 \\
\hline $\mathrm{C}$ & 2.86203700 & 2.97797800 & -1.16747400 \\
\hline $\mathrm{C}$ & 3.57562000 & 2.43483000 & -2.23647600 \\
\hline $\mathrm{C}$ & 3.25808500 & 1.15117900 & -2.68663100 \\
\hline $\mathrm{C}$ & 2.23676300 & 0.45471300 & -2.04996100 \\
\hline $\mathrm{H}$ & 3.10490800 & 3.97077100 & -0.80794700 \\
\hline $\mathrm{H}$ & 4.36923500 & 3.00626300 & -2.70812500 \\
\hline $\mathrm{H}$ & 3.78964800 & 0.69367900 & -3.51433700 \\
\hline $\mathrm{H}$ & 1.95357500 & -0.54396700 & -2.36055300 \\
\hline $\mathrm{C}$ & 1.03215900 & 2.70633400 & 0.56866900 \\
\hline $\mathrm{C}$ & 1.17357100 & 3.95987200 & 1.16787400 \\
\hline $\mathrm{C}$ & 0.34829400 & 4.31159800 & 2.23660600 \\
\hline $\mathrm{H}$ & 1.91673900 & 4.66172900 & 0.80873300 \\
\hline $\mathrm{C}$ & -0.71136200 & 2.16911300 & 2.04925500 \\
\hline $\mathrm{C}$ & -0.61093600 & 3.40109700 & 2.68615600 \\
\hline $\mathrm{H}$ & 0.45266600 & 5.28383900 & 2.70848100 \\
\hline $\mathrm{H}$ & -1.43980100 & 1.42935500 & 2.35934300 \\
\hline $\mathrm{H}$ & -1.27176300 & 3.63698300 & 3.51357900 \\
\hline $\mathrm{N}$ & 1.54088600 & 0.98362500 & -1.02387800 \\
\hline $\mathrm{N}$ & 0.09281200 & 1.82570900 & 1.02348500 \\
\hline $\mathrm{Ru}$ & -0.00004100 & 0.00001800 & -0.00025700 \\
\hline \multicolumn{3}{|c|}{ Zero-point correction $=$} & 0.486553 (Hartree/Particle) \\
\hline \multicolumn{3}{|c|}{ Thermal correction to Energy= } & 0.515288 \\
\hline \multicolumn{3}{|c|}{ Thermal correction to Enthalpy= } & 0.516232 \\
\hline \multicolumn{4}{|c|}{ Thermal correction to Gibbs Free Energy= } \\
\hline \multicolumn{4}{|c|}{ Sum of electronic and zero-point Energies= } \\
\hline \multicolumn{3}{|c|}{ Sum of electronic and thermal Energies= } & -1578.979582 \\
\hline \multicolumn{3}{|c|}{ Sum of electronic and thermal Enthalpies } & -1578.978637 \\
\hline \multicolumn{4}{|c|}{ Sum of electronic and thermal Free Energies= } \\
\hline
\end{tabular}

(U)B3LYP-D3/6-311++G(2d,p)-SDD/SMD//(U)B3LYP-D3/6-31+G(d)-LANL2DZ energy = -1581.39955

$\begin{array}{cccc}\mathbf{R u}(\mathbf{b p y}) \mathbf{3}^{+} & & & \\ \mathrm{C} & -1.48958900 & 2.46638700 & -0.59564700 \\ \mathrm{C} & -1.89498500 & 3.63713200 & -1.26282600 \\ \mathrm{C} & -1.21087800 & 4.06677200 & -2.38908600 \\ \mathrm{C} & -0.11322600 & 3.31826800 & -2.84736000 \\ \mathrm{C} & 0.23686700 & 2.16640000 & -2.15849800 \\ \mathrm{H} & -2.74446300 & 4.20250200 & -0.89775500 \\ \mathrm{H} & -1.52117300 & 4.96888100 & -2.90673000 \\ \mathrm{H} & 0.45684400 & 3.62168200 & -3.71854400 \\ \mathrm{H} & 1.06955000 & 1.54852600 & -2.47430400 \\ \mathrm{C} & -2.13332900 & 1.93636700 & 0.59670100 \\ \mathrm{C} & -3.20432100 & 2.55907100 & 1.26403300 \\ \mathrm{C} & -3.75706000 & 1.97031700 & 2.39049200 \\ \mathrm{H} & -3.59614000 & 3.50126300 & 0.89896000 \\ \mathrm{C} & -2.17010900 & 0.18457500 & 2.15970900 \\ \mathrm{C} & -3.23311500 & 0.74943900 & 2.84880100 \\ \mathrm{H} & -4.58269600 & 2.44810200 & 2.90826300 \\ \mathrm{H} & -1.72354500 & -0.75121300 & 2.47552400 \\ \mathrm{H} & -3.64009900 & 0.24835200 & 3.72016500 \\ \mathrm{~N} & -0.42733600 & 1.73517900 & -1.06838100 \\ \mathrm{~N} & -1.61969800 & 0.75346500 & 1.06935400\end{array}$




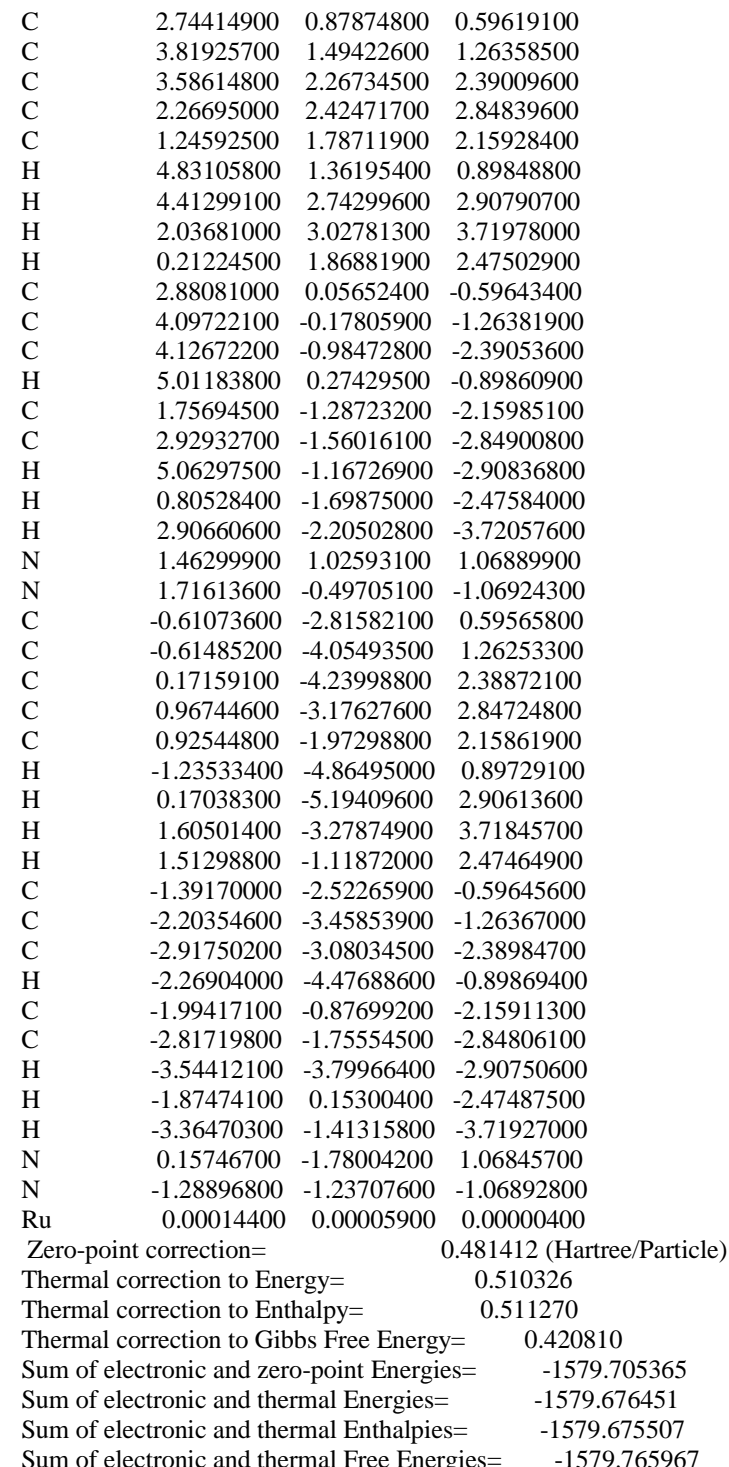

(U)B3LYP-D3/6-311++G(2d,p)-SDD/SMD//(U)B3LYP-D3/6-31+G(d)-LANL2DZ energy = -1581.696181

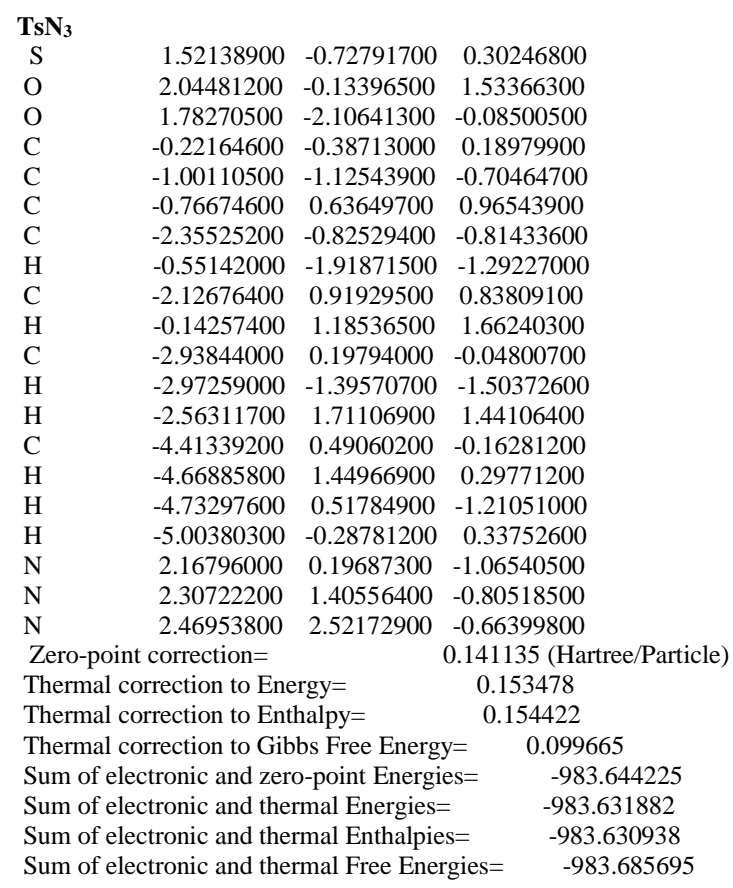




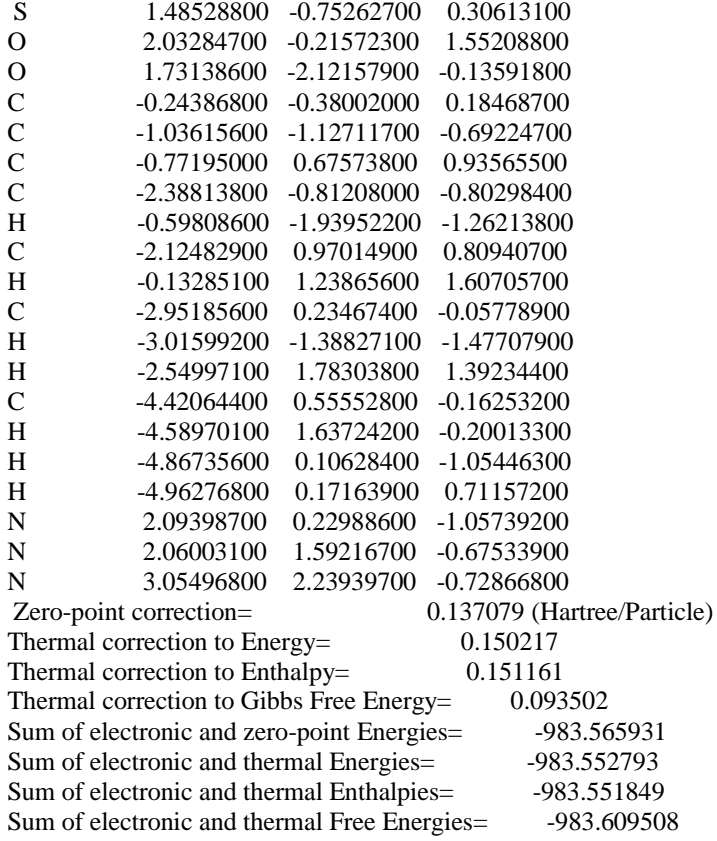

${ }^{3}$ INT1

$\begin{array}{llll}\mathrm{Ni} & 0.60247500 & -0.06850300 & 0.37139500\end{array}$

$\begin{array}{llll}\mathrm{Cl} & -0.64746600 & 0.60562200 & 2.13150000\end{array}$

$\begin{array}{llll}\mathrm{Cl} & 2.61559600 & -0.53465600 & -0.42318900\end{array}$

$\begin{array}{llll}\mathrm{C} & -1.52309700 & 0.59724600 & -1.42751800\end{array}$

$\begin{array}{llll}\mathrm{C} & -1.23516800 & -0.87750500 & -1.64700300\end{array}$

$\begin{array}{llll}\mathrm{H} & -1.86859200 & 1.06628900 & -2.35761100\end{array}$

$\begin{array}{llll}\mathrm{H} & -2.26456900 & 0.75724500 & -0.63450600\end{array}$

$\mathrm{H} \quad-0.45285700 \quad-1.00307400 \quad-2.39999800$

$\begin{array}{lllll}\mathrm{H} & -2.13638800 & -1.41547900 & -1.95908900\end{array}$

$\begin{array}{llll}\mathrm{O} & -0.71345900 & -1.46310100 & -0.43888100\end{array}$

$\begin{array}{lllll}\mathrm{O} & -0.27419700 & 1.18521300 & -1.03708200\end{array}$

$\begin{array}{llll}\text { C } & -1.70273300 & -2.06799700 & 0.41818200\end{array}$

$\mathrm{H} \quad-2.16516200 \quad-2.90006100 \quad-0.12314100$

$\begin{array}{llll}\mathrm{H} & -1.16961100 & -2.43446700 & 1.29423400\end{array}$

$\begin{array}{llll}\mathrm{H} & -2.44834700 & -1.33439800 & 0.73550900\end{array}$

$\begin{array}{llll}\text { C } & -0.35050000 & 2.58621400 & -0.71915800\end{array}$

$\begin{array}{llll}\mathrm{H} & -1.00945900 & 2.74206600 & 0.13999400\end{array}$

$\begin{array}{llll}\mathrm{H} & 0.66512200 & 2.89872200 & -0.47459900\end{array}$

$\mathrm{H} \quad-0.70740500 \quad 3.13015600 \quad-1.60047200$

Zero-point correction $=\quad 0.147757$ (Hart

Thermal correction to Energy= $\quad 0.160578$

Thermal correction to Enthalpy= $\quad 0.161523$

Thermal correction to Gibbs Free Energy $=0.105893$

Sum of electronic and zero-point Energies $=\quad-1398.571276$

Sum of electronic and thermal Energies $=\quad-1398.558454$

Sum of electronic and thermal Enthalpies $=\quad-1398.557510$

Sum of electronic and thermal Free Energies $=\quad-1398.613139$

(U)B3LYP-D3/6-311++G(2d,p)-SDD/SMD//(U)B3LYP-D3/6-31+G(d)-LANL2DZ energy = -1400.569366

$\begin{array}{crrr}{ }^{2} \mathbf{I N T 2} & & & \\ \mathrm{Ni} & -0.64474400 & -0.00088800 & -0.00003900 \\ \mathrm{Cl} & -2.81490400 & -0.00134200 & 0.00005800 \\ \mathrm{C} & 2.18199600 & -0.72786500 & 0.20278100 \\ \mathrm{C} & 2.18066800 & 0.73141000 & -0.20262800 \\ \mathrm{H} & 3.04842500 & -1.24580700 & -0.23040000 \\ \mathrm{H} & 2.20964100 & -0.82671400 & 1.29690200 \\ \mathrm{H} & 2.20821500 & 0.83030000 & -1.29674800 \\ \mathrm{H} & 3.04612100 & 1.25093700 & 0.23061000 \\ \mathrm{O} & 0.96917700 & 1.29507900 & 0.30087400 \\ \mathrm{O} & 0.97157500 & -1.29378700 & -0.30080800 \\ \mathrm{C} & 0.77755900 & 2.67167100 & -0.04281200 \\ \mathrm{H} & 0.74433000 & 2.79358500 & -1.13249600 \\ \mathrm{H} & -0.17777800 & 2.96772600 & 0.39078100 \\ \mathrm{H} & 1.59001600 & 3.27543800 & 0.38053700 \\ \mathrm{C} & 0.78266200 & -2.67080700 & 0.04264300 \\ \mathrm{H} & 0.74958400 & -2.79297300 & 1.13230400\end{array}$




\begin{tabular}{|c|c|c|}
\hline $\mathrm{H}$ & $-0.17203800-2.96871000 \quad-0$ & 9300 \\
\hline $\mathrm{H}$ & $\begin{array}{lll}1.59635100 & -3.27289400 & -0.3\end{array}$ & 73300 \\
\hline \multicolumn{2}{|c|}{ Zero-point correction= } & 7 (Hartree/Particle) \\
\hline \multicolumn{2}{|c|}{ Thermal correction to Energy= } & 57006 \\
\hline \multicolumn{2}{|c|}{ Thermal correction to Enthalpy= } & 57950 \\
\hline \multicolumn{2}{|c|}{ Thermal correction to Gibbs Free Energy= } & 0.104583 \\
\hline \multicolumn{2}{|c|}{ Sum of electronic and zero-point Energies= } & -938.325055 \\
\hline \multicolumn{2}{|c|}{ Sum of electronic and thermal Energies= } & -938.313686 \\
\hline \multicolumn{2}{|c|}{ Sum of electronic and thermal Enthalpies= } & -938.312742 \\
\hline \multicolumn{2}{|c|}{ Sum of electronic and thermal Free Energies } & -938.366109 \\
\hline
\end{tabular}

(U)B3LYP-D3/6-311++G(2d,p)-SDD/SMD//(U)B3LYP-D3/6-31+G(d)-LANL2DZ energy = -940.2727584

\begin{tabular}{|c|c|c|c|}
\hline \multicolumn{4}{|c|}{${ }^{2}$ INT3 } \\
\hline $\mathrm{C}$ & 2.41707500 & 1.50633700 & 1.72470400 \\
\hline $\mathrm{C}$ & 2.02507500 & 2.41141700 & 0.57425900 \\
\hline $\mathrm{H}$ & 3.18785000 & 1.99635100 & 2.33370700 \\
\hline $\mathrm{H}$ & 1.55748900 & 1.25299200 & 2.35887200 \\
\hline $\mathrm{H}$ & 2.89817900 & 2.62736000 & -0.05264400 \\
\hline $\mathrm{H}$ & 1.58676200 & 3.34591400 & 0.94615000 \\
\hline $\mathrm{O}$ & 1.04589900 & 1.71458000 & -0.21596200 \\
\hline $\mathrm{O}$ & 2.95435500 & 0.32017000 & 1.13728700 \\
\hline $\mathrm{C}$ & 0.73075000 & 2.37616500 & -1.45915000 \\
\hline $\mathrm{H}$ & 1.63357400 & 2.46701000 & -2.06950300 \\
\hline $\mathrm{H}$ & 0.00511700 & 1.74044800 & -1.96559800 \\
\hline $\mathrm{H}$ & 0.29698800 & 3.35811000 & -1.23862800 \\
\hline $\mathrm{C}$ & 3.56481700 & -0.60115200 & 2.05043200 \\
\hline $\mathrm{H}$ & 2.83186700 & -0.96121500 & 2.77892100 \\
\hline $\mathrm{H}$ & 3.94033600 & -1.42592800 & 1.44336000 \\
\hline $\mathrm{H}$ & 4.40291400 & -0.10478800 & 2.55346900 \\
\hline $\mathrm{Ni}$ & 1.69348800 & -0.27139700 & -0.46255700 \\
\hline S & -0.81517600 & -1.52758800 & -0.51063100 \\
\hline $\mathrm{O}$ & -1.20909600 & -2.92933200 & -0.64398600 \\
\hline $\mathrm{O}$ & -0.02333200 & -0.89375900 & -1.63272900 \\
\hline $\mathrm{C}$ & -2.28781100 & -0.56078400 & -0.22788900 \\
\hline $\mathrm{C}$ & -3.53349900 & -1.14984400 & -0.43204800 \\
\hline $\mathrm{C}$ & -2.16956400 & 0.77409000 & 0.17221200 \\
\hline $\mathrm{C}$ & -4.68245700 & -0.37970800 & -0.23997600 \\
\hline $\mathrm{H}$ & -3.59594200 & -2.19111400 & -0.72870700 \\
\hline $\mathrm{C}$ & -3.32667300 & 1.52271700 & 0.36064700 \\
\hline $\mathrm{H}$ & -1.19000600 & 1.20805600 & 0.34083000 \\
\hline $\mathrm{C}$ & -4.59924500 & 0.96086000 & 0.15663900 \\
\hline $\mathrm{H}$ & -5.65776500 & -0.83219300 & -0.39823700 \\
\hline $\mathrm{H}$ & -3.24563000 & 2.56018700 & 0.67673700 \\
\hline $\mathrm{C}$ & -5.84153400 & 1.78809000 & 0.37724900 \\
\hline $\mathrm{H}$ & -5.80626400 & 2.71812000 & -0.20261100 \\
\hline $\mathrm{H}$ & -6.74332100 & 1.24130400 & 0.08573300 \\
\hline $\mathrm{H}$ & -5.94476100 & 2.06742500 & 1.43339600 \\
\hline $\mathrm{N}$ & 0.26400900 & -1.15853400 & 0.69466300 \\
\hline $\mathrm{N}$ & -0.17562000 & -1.46446600 & 1.97474000 \\
\hline $\mathrm{N}$ & 0.40027000 & -1.20347800 & 2.97589300 \\
\hline $\mathrm{Cl}$ & 3.30134800 & 0.01032100 & -2.01117400 \\
\hline
\end{tabular}

Zero-point correction $=\quad 0.287832$ (Hartree

Thermal correction to Energy=

Thermal correction to Enthalpy= $\quad 0.313307$

Thermal correction to Gibbs Free Energy $=0.230398$

Sum of electronic and zero-point Energies $=\quad-1921.988800$

Sum of electronic and thermal Energies $=\quad-1921.964270$

Sum of electronic and thermal Enthalpies $=\quad-1921.963326$

Sum of electronic and thermal Free Energies $=\quad-1922.046234$

(U)B3LYP-D3/6-311++G(2d,p)-SDD/SMD//(U)B3LYP-D3/6-31+G(d)-LANL2DZ energy = -1924.3106261

$\begin{array}{cccc}{ }^{2} \mathbf{T S 1} & & & \\ \mathrm{C} & 2.43490000 & 1.12958800 & 1.99234200 \\ \mathrm{C} & 2.05792700 & 2.25438900 & 1.04985700 \\ \mathrm{H} & 3.20930900 & 1.47271700 & 2.69081100 \\ \mathrm{H} & 1.56448400 & 0.77155700 & 2.55877600 \\ \mathrm{H} & 2.93022300 & 2.56959300 & 0.46523800 \\ \mathrm{H} & 1.64844100 & 3.10605500 & 1.60763100 \\ \mathrm{O} & 1.05272600 & 1.74927800 & 0.15613200 \\ \mathrm{O} & 2.95415400 & 0.07471500 & 1.18257300 \\ \mathrm{C} & 0.71286900 & 2.66198700 & -0.90820600 \\ \mathrm{H} & 1.60066600 & 2.87642300 & -1.50977500 \\ \mathrm{H} & -0.03262100 & 2.15179500 & -1.51721000 \\ \mathrm{H} & 0.29538800 & 3.57638500 & -0.47145900 \\ \mathrm{C} & 3.48119600 & -1.04421700 & 1.90395200 \\ \mathrm{H} & 2.70523900 & -1.50502400 & 2.52365000 \\ \mathrm{H} & 3.83715600 & -1.75192800 & 1.15426900 \\ \mathrm{H} & 4.32224300 & -0.71069200 & 2.52322000 \\ \mathrm{Ni} & 1.69130600 & -0.14542100 & -0.53053500 \\ \mathrm{~S} & -0.79579900 & -1.36470200 & -0.80757100\end{array}$




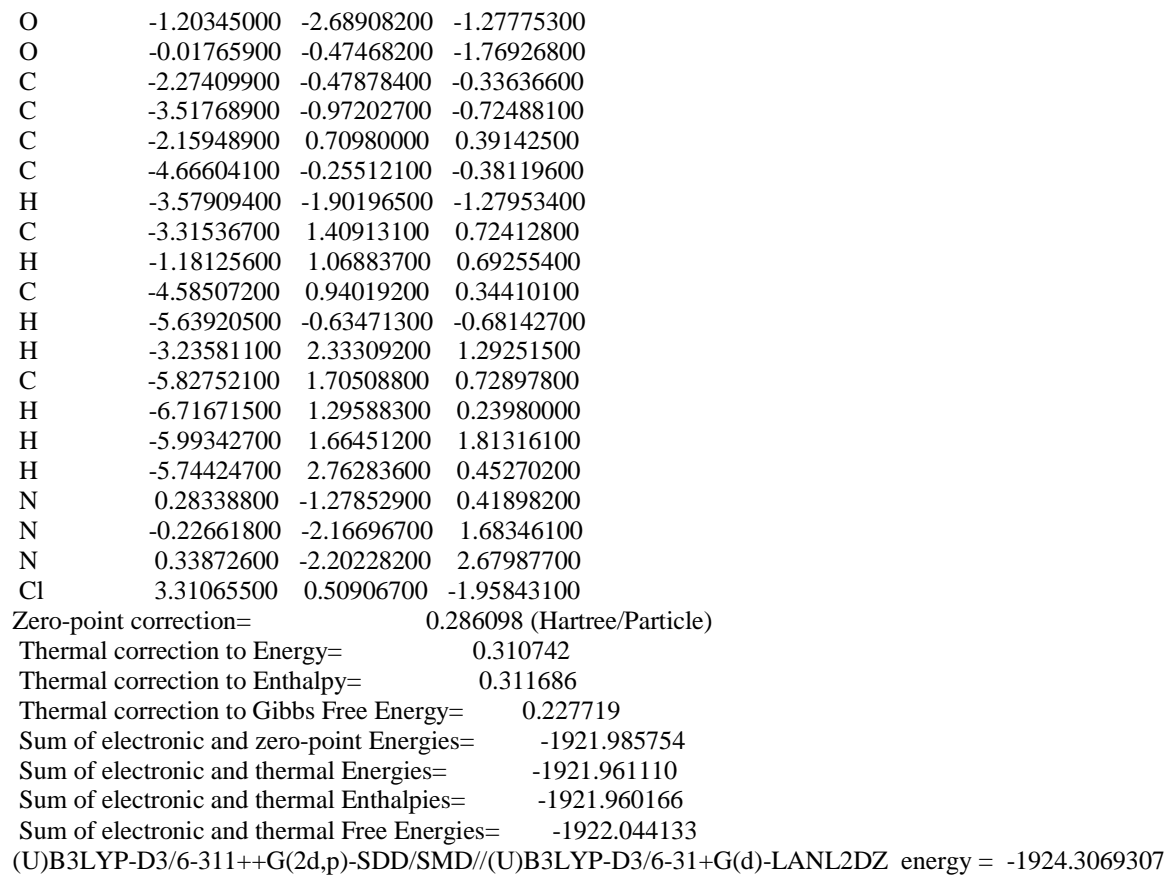

\section{${ }^{2}$ INT4}

\begin{tabular}{|c|c|c|c|}
\hline $\mathrm{C}$ & 2.30044600 & 1.74440800 & 1.64124000 \\
\hline $\mathrm{C}$ & 2.11079400 & 2.38828300 & 0.28423500 \\
\hline $\mathrm{H}$ & 2.98329700 & 2.34717100 & 2.25402300 \\
\hline $\mathrm{H}$ & 1.33884000 & 1.63099800 & 2.15862800 \\
\hline $\mathrm{H}$ & 3.06130600 & 2.42549600 & -0.26149300 \\
\hline $\mathrm{H}$ & 1.69785000 & 3.39926700 & 0.39086200 \\
\hline $\mathrm{O}$ & 1.17911700 & 1.57411000 & -0.44567300 \\
\hline $\mathrm{O}$ & 2.87938900 & 0.45531000 & 1.41333600 \\
\hline $\mathrm{C}$ & 1.01324400 & 1.96796700 & -1.82321900 \\
\hline $\mathrm{H}$ & 1.97309600 & 1.91300900 & -2.34452500 \\
\hline $\mathrm{H}$ & 0.31367600 & 1.25487900 & -2.25816500 \\
\hline $\mathrm{H}$ & 0.60122800 & 2.98325700 & -1.85277200 \\
\hline $\mathrm{C}$ & 3.02161000 & -0.33513500 & 2.60046600 \\
\hline $\mathrm{H}$ & 2.03824400 & -0.56303600 & 3.02544800 \\
\hline $\mathrm{H}$ & 3.52381600 & -1.25484700 & 2.29762100 \\
\hline $\mathrm{H}$ & 3.64385900 & 0.20599500 & 3.32290900 \\
\hline $\mathrm{Ni}$ & 1.75357400 & -0.44274200 & -0.19613800 \\
\hline S & -0.73431900 & -1.49414100 & 0.01637300 \\
\hline $\mathrm{O}$ & -1.02253400 & -2.92133000 & 0.21542600 \\
\hline $\mathrm{O}$ & -0.04546400 & -1.06593400 & -1.27881100 \\
\hline $\mathrm{C}$ & -2.28535000 & -0.60930600 & 0.03824900 \\
\hline $\mathrm{C}$ & -3.48051800 & -1.31294600 & 0.15577400 \\
\hline $\mathrm{C}$ & -2.27184300 & 0.78610400 & -0.05840600 \\
\hline $\mathrm{C}$ & -4.68473600 & -0.60323900 & 0.16914100 \\
\hline $\mathrm{H}$ & -3.46278000 & -2.39463800 & 0.23320600 \\
\hline $\mathrm{C}$ & -3.47970200 & 1.47481400 & -0.04267400 \\
\hline $\mathrm{H}$ & -1.32813900 & 1.31619700 & -0.13569700 \\
\hline $\mathrm{C}$ & -4.70477000 & 0.79268400 & 0.06880400 \\
\hline $\mathrm{H}$ & -5.62139700 & -1.14749200 & 0.25790900 \\
\hline $\mathrm{H}$ & -3.47795000 & 2.56000000 & -0.11700000 \\
\hline $\mathrm{C}$ & -6.00478100 & 1.55907600 & 0.07868800 \\
\hline $\mathrm{H}$ & -6.86218600 & 0.89026800 & 0.20134200 \\
\hline $\mathrm{H}$ & -6.02738000 & 2.29040400 & 0.89598300 \\
\hline $\mathrm{H}$ & -6.14252300 & 2.11454300 & -0.85740900 \\
\hline $\mathrm{N}$ & 0.28457700 & -0.87829400 & 1.10611400 \\
\hline $\mathrm{Cl}$ & 3.48669000 & -0.52787600 & -1.62540000 \\
\hline
\end{tabular}

Zero-point correction= -0.279409 (Hart

Thermal correction to Energy $=\quad 0.301657$

Thermal correction to Enthalpy= $\quad 0.302602$

Thermal correction to Gibbs Free Energy $=0.224986$

Sum of electronic and zero-point Energies $=\quad-1812.496549$

Sum of electronic and thermal Energies $=\quad-1812.474300$

Sum of electronic and thermal Enthalpies $=\quad-1812.473356$

Sum of electronic and thermal Free Energies $=\quad-1812.550972$

(U)B3LYP-D3/6-311++G(2d,p)-SDD/SMD//(U)B3LYP-D3/6-31+G(d)-LANL2DZ energy = -1814.7841562

\section{${ }^{4}$ INT4}

$\begin{array}{llll}\mathrm{C} & 2.02029700 & 1.87963700 & 1.60127500\end{array}$

C $\quad 1.88269500 \quad 2.48222900 \quad 0.21899000$ 


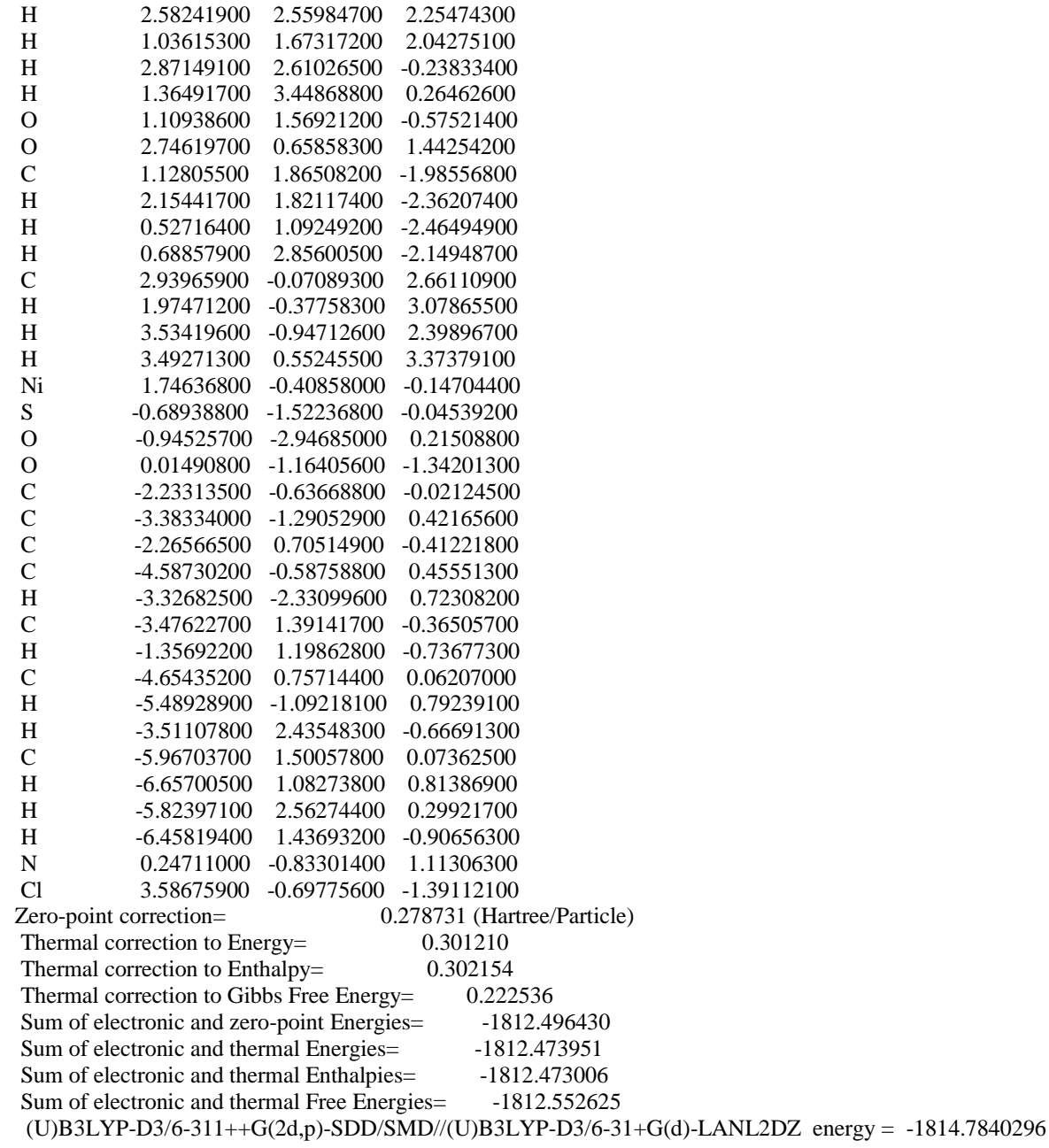

\begin{tabular}{lccc}
\multicolumn{4}{c}{ alkyltrifluoroborate $\left(\mathbf{2}^{-}\right)$} \\
$\mathrm{B}$ & -1.01278900 & 0.03965300 & 0.00000000 \\
$\mathrm{~F}$ & -1.23826200 & -0.77719700 & -1.16068500 \\
$\mathrm{~F}$ & -1.98853300 & 1.09067400 & 0.00000000 \\
$\mathrm{~F}$ & -1.23826200 & -0.77719700 & 1.16068500 \\
$\mathrm{C}$ & 0.49875000 & 0.65211800 & 0.00000000 \\
$\mathrm{H}$ & 0.62401200 & 1.30454800 & -0.88218000 \\
$\mathrm{H}$ & 0.62401200 & 1.30454800 & 0.88218000 \\
$\mathrm{C}$ & 1.60834600 & -0.40654600 & 0.00000000 \\
$\mathrm{H}$ & 1.48313000 & -1.05726700 & 0.87779300 \\
$\mathrm{H}$ & 1.48313000 & -1.05726700 & -0.87779300 \\
$\mathrm{C}$ & 3.03204600 & 0.17001300 & 0.00000000 \\
$\mathrm{H}$ & 3.80347400 & -0.61616400 & 0.00000000 \\
$\mathrm{H}$ & 3.19842400 & 0.80165400 & -0.88469700 \\
$\mathrm{H}$ & 3.19842400 & 0.80165400 & 0.88469700 \\
Zero-point correction= & $0.104756(\mathrm{Hartree} / \mathrm{Particle})$ \\
Thermal correction to Energy= & 0.112895 \\
Thermal correction to Enthalpy= & 0.113840 \\
Thermal correction to Gibbs Free Energy= & 0.071723 \\
Sum of electronic and zero-point Energies= & -443.104012 \\
Sum of electronic and thermal Energies= & -443.095873 \\
Sum of electronic and thermal Enthalpies= & -443.094929 \\
Sum of electronic and thermal Free Energies= & -443.137046
\end{tabular}

B3LYP-D3/6-311++G(2d,p)/SMD//B3LYP-D3/6-31+G(d) energy = -443.4303845

\begin{tabular}{cccc}
\multicolumn{4}{l}{ alkyl radical } \\
$\mathrm{C}$ & -1.33922800 & -0.07924300 & 0.00000000 \\
$\mathrm{H}$ & -1.80165900 & -0.40268800 & 0.92848800 \\
$\mathrm{H}$ & -1.80165900 & -0.40268800 & -0.92848800 \\
$\mathrm{C}$ & 0.00000000 & 0.57934000 & 0.00000000 \\
$\mathrm{H}$ & 0.09975200 & 1.22712200 & -0.88191600 \\
$\mathrm{H}$ & 0.09975200 & 1.22712200 & 0.88191600 \\
$\mathrm{C}$ & 1.17265600 & -0.43168900 & 0.00000000 \\
$\mathrm{H}$ & 2.13671500 & 0.09255900 & 0.00000000 \\
$\mathrm{H}$ & 1.13326800 & -1.07593900 & 0.88589000 \\
$\mathrm{H}$ & 1.13326800 & -1.07593900 & -0.88589000
\end{tabular}




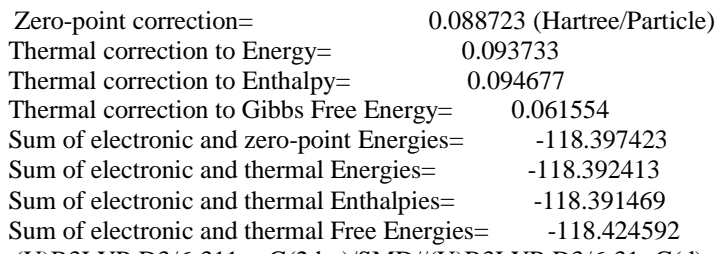

(U)B3LYP-D3/6-311++G(2d,p)/SMD//(U)B3LYP-D3/6-31+G(d) energy = -118.5228172

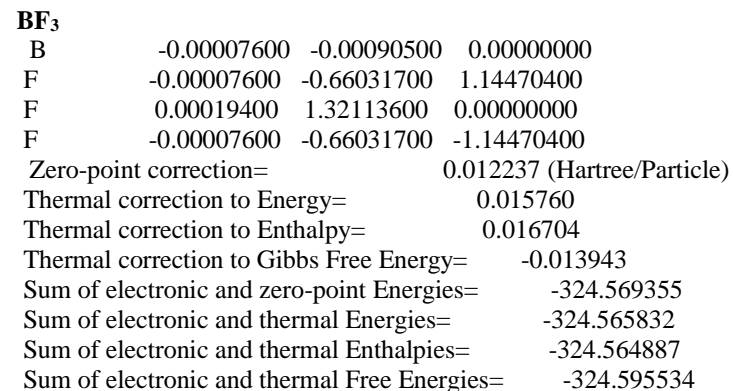

B3LYP-D3/6-311++G(2d,p)/SMD//B3LYP-D3/6-31+G(d) energy = -324.6848512

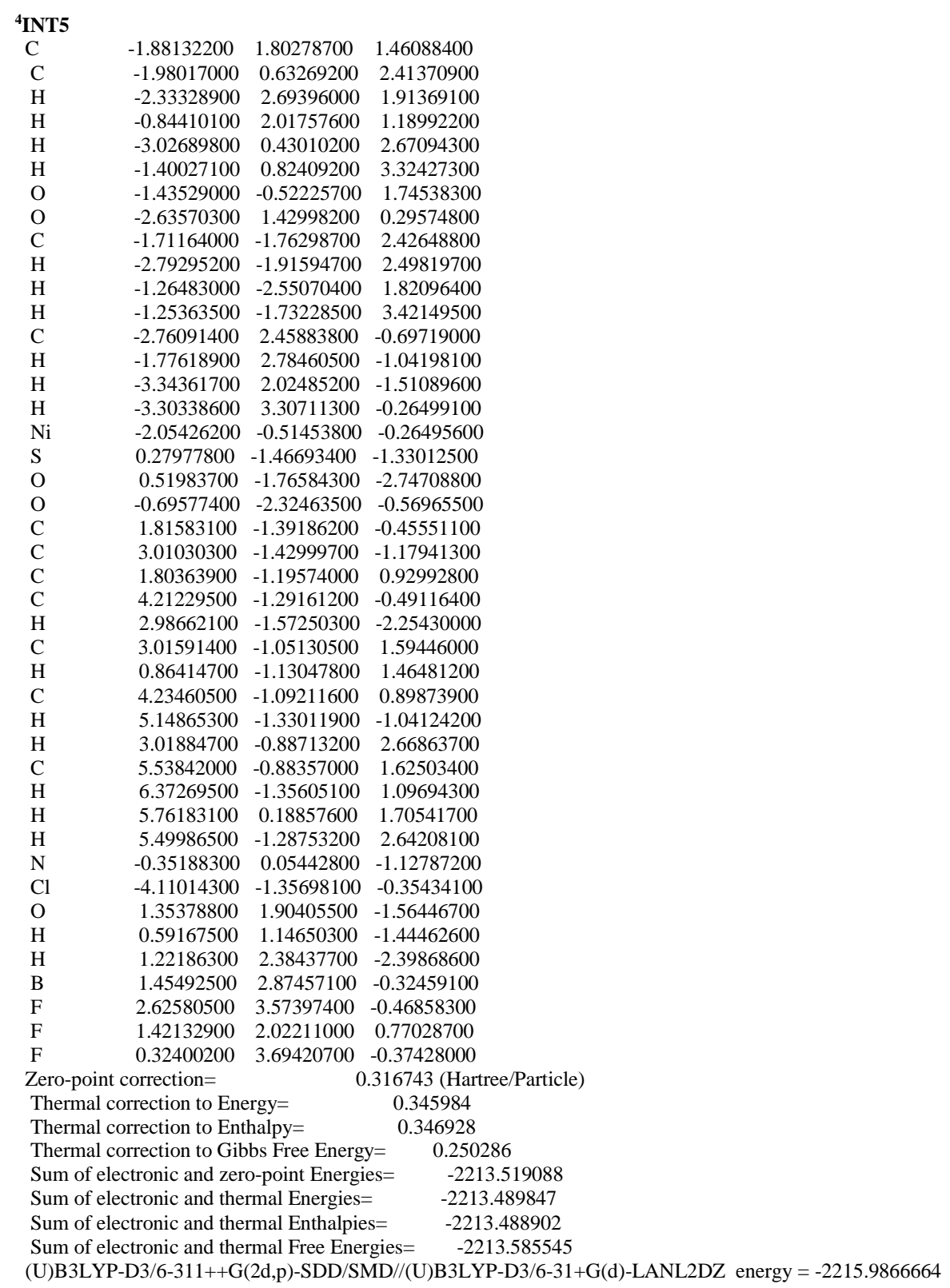

${ }^{3}$ INT6 


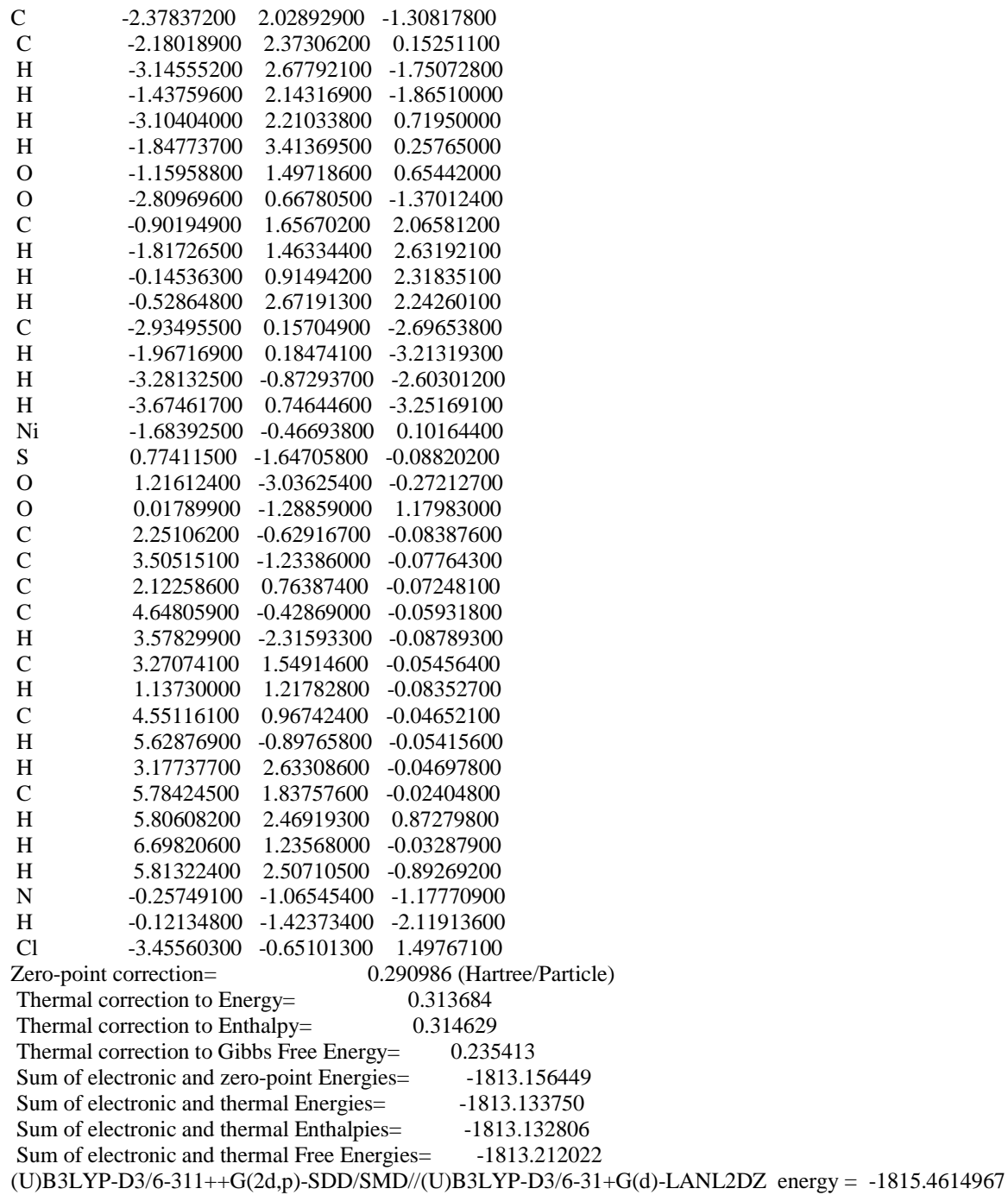

$\begin{array}{cccc}{ }^{2} \text { INT7 } & & & \\ \mathrm{C} & -1.11054400 & -2.81600000 & 1.32153400 \\ \mathrm{C} & -0.86029200 & -3.09837200 & -0.14317200 \\ \mathrm{H} & -1.49388200 & -3.71774700 & 1.81870300 \\ \mathrm{H} & -0.18251000 & -2.49243200 & 1.81209500 \\ \mathrm{H} & -1.80242100 & -3.31332300 & -0.66312300 \\ \mathrm{H} & -0.17979900 & -3.95654900 & -0.24249900 \\ \mathrm{O} & -0.25364500 & -1.93822300 & -0.70835600 \\ \mathrm{O} & -2.08801100 & -1.77522900 & 1.42143300 \\ \mathrm{C} & 0.01270600 & -2.05771600 & -2.11359200 \\ \mathrm{H} & -0.91805000 & -2.23936300 & -2.65925100 \\ \mathrm{H} & 0.44183600 & -1.10455400 & -2.42011100 \\ \mathrm{H} & 0.72736300 & -2.87498000 & -2.27759800 \\ \mathrm{C} & -2.33313900 & -1.37608700 & 2.76603100 \\ \mathrm{H} & -1.42314200 & -0.96146300 & 3.21965500 \\ \mathrm{H} & -3.11617300 & -0.61758200 & 2.73571200 \\ \mathrm{H} & -2.68074000 & -2.23511600 & 3.35426200 \\ \mathrm{C} & -3.76064900 & 3.55463800 & 0.01944700 \\ \mathrm{C} & -2.72359700 & 2.48331900 & -0.38639000 \\ \mathrm{H} & -3.57301000 & 3.92794700 & 1.03348900 \\ \mathrm{H} & -4.78047300 & 3.15406100 & -0.01585600 \\ \mathrm{C} & -2.79607500 & 1.31712800 & 0.56187600 \\ \mathrm{H} & -2.92829600 & 2.14283000 & -1.40576900 \\ \mathrm{H} & -1.72432500 & 2.92690200 & -0.37666600 \\ \mathrm{H} & -2.43772300 & 1.52369400 & 1.57145500 \\ \mathrm{H} & -3.73700000 & 0.76726800 & 0.54964000 \\ \mathrm{H} & -3.70469100 & 4.40576800 & -0.66922200 \\ \mathrm{Ni} & -1.54640200 & -0.16777400 & -0.07729900 \\ \mathrm{~S} & 0.77492700 & 1.41762100 & 0.04459300 \\ \mathrm{O} & 0.83277600 & 2.84748600 & 0.40455400 \\ \mathrm{O} & 0.18227800 & 1.03537100 & -1.27903400 \\ \mathrm{C} & 2.45682000 & 0.80245900 & 0.06879100 \\ \mathrm{C} & 3.51361900 & 1.70481100 & 0.15520400\end{array}$




\begin{tabular}{lrrr}
$\mathrm{C}$ & 2.68583300 & -0.57386500 & -0.02528100 \\
$\mathrm{C}$ & 4.82393000 & 1.21767300 & 0.14280800 \\
$\mathrm{H}$ & 3.30817500 & 2.76700000 & 0.23286200 \\
$\mathrm{C}$ & 3.99685200 & -1.03942300 & -0.03558900 \\
$\mathrm{H}$ & 1.84436800 & -1.25597100 & -0.08129400 \\
$\mathrm{C}$ & 5.08613400 & -0.15392700 & 0.04719800 \\
$\mathrm{H}$ & 5.65271800 & 1.91807700 & 0.20920700 \\
$\mathrm{H}$ & 4.18334700 & -2.10896000 & -0.10692100 \\
$\mathrm{C}$ & 6.50052200 & -0.68101000 & 0.03337000 \\
$\mathrm{H}$ & 6.70319800 & -1.24590300 & -0.88504100 \\
$\mathrm{H}$ & 7.23068300 & 0.13188500 & 0.09584500 \\
$\mathrm{H}$ & 6.67914000 & -1.35955100 & 0.87693500 \\
$\mathrm{~N}$ & -0.08296700 & 0.49804000 & 1.08855400 \\
$\mathrm{H}$ & -0.23637900 & 1.01963300 & 1.95114400 \\
$\mathrm{Cl}$ & -3.10064200 & -0.80759600 & -1.58875500 \\
Zero-point correction= & \multicolumn{2}{c}{$0.386030(\mathrm{Hartree} / \mathrm{Particle})$} \\
Thermal correction to Energy= & 0.413524 \\
Thermal correction to Enthalpy= & 0.414468 \\
Thermal correction to Gibbs Free Energy= & 0.326025 \\
Sum of electronic and zero-point Energies= & -1931.588043 \\
Sum of electronic and thermal Energies= & -1931.560550 \\
Sum of electronic and thermal Enthalpies= & -1931.559606 \\
Sum of electronic and thermal Free Energies= & -1931.648049 \\
Sum &
\end{tabular}

(U)B3LYP-D3/6-311++G(2d,p)-SDD/SMD//(U)B3LYP-D3/6-31+G(d)-LANL2DZ energy = -1934.01439437

\begin{tabular}{|c|c|c|c|}
\hline \multicolumn{4}{|c|}{${ }^{2}$ TS2 } \\
\hline $\mathrm{C}$ & -0.49141700 & -2.97937400 & 1.01706700 \\
\hline $\mathrm{C}$ & -0.81579800 & -3.13466000 & -0.45356600 \\
\hline $\mathrm{H}$ & -0.73989900 & -3.90282700 & 1.55818500 \\
\hline $\mathrm{H}$ & 0.57793000 & -2.75964100 & 1.15127700 \\
\hline $\mathrm{H}$ & -1.89624100 & -3.26006900 & -0.59781500 \\
\hline $\mathrm{H}$ & -0.28517500 & -4.01339000 & -0.85168800 \\
\hline $\mathrm{O}$ & -0.37467400 & -1.95349700 & -1.11422700 \\
\hline $\mathrm{O}$ & -1.26611900 & -1.90106700 & 1.55195500 \\
\hline $\mathrm{C}$ & -0.69028600 & -1.92009800 & -2.51115800 \\
\hline $\mathrm{H}$ & -1.77435800 & -1.97852500 & -2.65295500 \\
\hline $\mathrm{H}$ & -0.32128400 & -0.96212100 & -2.88018300 \\
\hline $\mathrm{H}$ & -0.18549200 & -2.74784200 & -3.02861800 \\
\hline $\mathrm{C}$ & -0.94961600 & -1.60203900 & 2.90427500 \\
\hline $\mathrm{H}$ & 0.09478600 & -1.27168900 & 2.99767900 \\
\hline $\mathrm{H}$ & -1.62346300 & -0.80377800 & 3.22100400 \\
\hline $\mathrm{H}$ & -1.11173600 & -2.48425400 & 3.53728200 \\
\hline $\mathrm{C}$ & -3.95070100 & 3.03511300 & 0.16941000 \\
\hline $\mathrm{C}$ & -2.55414400 & 2.45359400 & -0.12113300 \\
\hline $\mathrm{H}$ & -3.94365600 & 3.68643500 & 1.05216600 \\
\hline $\mathrm{H}$ & -4.67541300 & 2.23012300 & 0.33076600 \\
\hline $\mathrm{C}$ & -2.07328100 & 1.69670000 & 1.08999100 \\
\hline $\mathrm{H}$ & -2.63088400 & 1.79432600 & -0.99112600 \\
\hline $\mathrm{H}$ & -1.84529100 & 3.24835800 & -0.36837400 \\
\hline $\mathrm{H}$ & -1.65741900 & 2.32280200 & 1.87463900 \\
\hline $\mathrm{H}$ & -2.78374800 & 0.96961900 & 1.49216700 \\
\hline $\mathrm{H}$ & -4.28879600 & 3.63028600 & -0.68653000 \\
\hline $\mathrm{Ni}$ & -1.55207300 & -0.30010000 & 0.11367000 \\
\hline $\mathrm{S}$ & 0.70286100 & 1.74302400 & -0.34777700 \\
\hline $\mathrm{O}$ & 0.88050900 & 3.16339400 & 0.00111400 \\
\hline $\mathrm{O}$ & 0.09059800 & 1.37411000 & -1.63619600 \\
\hline $\mathrm{C}$ & 2.30219000 & 0.95316800 & -0.18941600 \\
\hline $\mathrm{C}$ & 3.39385000 & 1.69944500 & 0.25196100 \\
\hline $\mathrm{C}$ & 2.42848900 & -0.40428400 & -0.50501900 \\
\hline $\mathrm{C}$ & 4.63658200 & 1.07224400 & 0.37479200 \\
\hline $\mathrm{H}$ & 3.26759700 & 2.75173100 & 0.48303100 \\
\hline $\mathrm{C}$ & 3.67546300 & -1.00828700 & -0.37636300 \\
\hline $\mathrm{H}$ & 1.56397500 & -0.97165400 & -0.83426500 \\
\hline $\mathrm{C}$ & 4.79780500 & -0.28349700 & 0.06290900 \\
\hline $\mathrm{H}$ & 5.49314400 & 1.64962600 & 0.71349500 \\
\hline $\mathrm{H}$ & 3.78438900 & -2.06196500 & -0.62444600 \\
\hline $\mathrm{C}$ & 6.14415500 & -0.95576400 & 0.17613800 \\
\hline $\mathrm{H}$ & 6.08028500 & -1.87907300 & 0.76434500 \\
\hline $\mathrm{H}$ & 6.53028600 & -1.22912700 & -0.81420700 \\
\hline $\mathrm{H}$ & 6.88036700 & -0.30070000 & 0.65218000 \\
\hline $\mathrm{N}$ & -0.22239600 & 0.94912200 & 0.79011300 \\
\hline $\mathrm{H}$ & 0.16257900 & 1.08086600 & 1.72281200 \\
\hline $\mathrm{Cl}$ & -3.56857100 & -0.94494400 & -0.71355700 \\
\hline \multicolumn{2}{|c|}{ Zero-point correction $=$} & & .384152 (Hartree/Particle) \\
\hline \multicolumn{3}{|c|}{ Thermal correction to Energy= } & 0.412013 \\
\hline \multicolumn{3}{|c|}{ Thermal correction to Enthalpy= } & 0.412957 \\
\hline \multicolumn{3}{|c|}{ Thermal correction to Gibbs Free Energy= } & 0.322233 \\
\hline \multicolumn{3}{|c|}{ Sum of electronic and zero-point Energies } & -1931.551768 \\
\hline \multicolumn{3}{|c|}{ Sum of electronic and thermal Energies= } & -1931.523907 \\
\hline \multicolumn{4}{|c|}{ Sum of electronic and thermal Enthalpies= } \\
\hline
\end{tabular}


Sum of electronic and thermal Free Energies $=\quad-1931.613686$

(U)B3LYP-D3/6-311++G(2d,p)-SDD/SMD//(U)B3LYP-D3/6-31+G(d)-LANL2DZ energy = -1933.97376537

\begin{tabular}{|c|c|c|c|}
\hline $\mathrm{C}$ & -1.00574000 & -3.01995200 & 1.34175800 \\
\hline $\mathrm{C}$ & -0.98066000 & -3.29851900 & -0.14481900 \\
\hline $\mathrm{H}$ & -1.52932700 & -3.82794800 & 1.87008200 \\
\hline $\mathrm{H}$ & 0.01707200 & -2.93357200 & 1.73655400 \\
\hline $\mathrm{H}$ & -1.99635600 & -3.37990300 & -0.54790800 \\
\hline $\mathrm{H}$ & -0.41589800 & -4.21739300 & -0.34782000 \\
\hline $\mathrm{O}$ & -0.31813900 & -2.18735200 & -0.76549300 \\
\hline $\mathrm{O}$ & -1.69837500 & -1.78635100 & 1.54157600 \\
\hline $\mathrm{C}$ & -0.21136100 & -2.31094700 & -2.20107100 \\
\hline $\mathrm{H}$ & -1.20952200 & -2.40599000 & -2.63688800 \\
\hline $\mathrm{H}$ & 0.26012200 & -1.39240700 & -2.54754500 \\
\hline $\mathrm{H}$ & 0.41069900 & -3.18321300 & -2.43186000 \\
\hline $\mathrm{C}$ & -1.70217100 & -1.35095800 & 2.89930400 \\
\hline $\mathrm{H}$ & -0.67701300 & -1.17741000 & 3.25255900 \\
\hline $\mathrm{H}$ & -2.26775200 & -0.42051300 & 2.93313200 \\
\hline $\mathrm{H}$ & -2.19122300 & -2.10426700 & 3.52908300 \\
\hline $\mathrm{C}$ & -4.01376900 & 3.48131200 & 0.10893100 \\
\hline $\mathrm{C}$ & -2.64399600 & 2.78301700 & 0.02876900 \\
\hline $\mathrm{H}$ & -4.08349000 & 4.12605100 & 0.99424900 \\
\hline $\mathrm{H}$ & -4.82703600 & 2.74757600 & 0.15724400 \\
\hline $\mathrm{C}$ & -2.38200900 & 1.91787200 & 1.22823300 \\
\hline $\mathrm{H}$ & -2.61337800 & 2.16469200 & -0.87632500 \\
\hline $\mathrm{H}$ & -1.84545400 & 3.52669200 & -0.06632800 \\
\hline $\mathrm{H}$ & -2.20017600 & 2.42802500 & 2.17450100 \\
\hline $\mathrm{H}$ & -3.02654700 & 1.04134900 & 1.32359100 \\
\hline $\mathrm{H}$ & -4.17674600 & 4.10664900 & -0.77759900 \\
\hline $\mathrm{Ni}$ & -1.28052100 & -0.41331000 & -0.14096300 \\
\hline S & 0.67483400 & 1.53430300 & -0.31761400 \\
\hline $\mathrm{O}$ & 0.76849400 & 2.99222400 & -0.52239700 \\
\hline $\mathrm{O}$ & -0.01925200 & 0.70977400 & -1.40927200 \\
\hline $\mathrm{C}$ & 2.35856500 & 0.90949500 & -0.24348000 \\
\hline $\mathrm{C}$ & 3.39828900 & 1.79396600 & 0.07099000 \\
\hline $\mathrm{C}$ & 2.59583700 & -0.47334300 & -0.30287400 \\
\hline $\mathrm{C}$ & 4.68059700 & 1.28901800 & 0.29071500 \\
\hline $\mathrm{H}$ & 3.20051500 & 2.86001000 & 0.10992700 \\
\hline $\mathrm{C}$ & 3.88288300 & -0.95293700 & -0.08607700 \\
\hline $\mathrm{H}$ & 1.78561400 & -1.15672000 & -0.53043900 \\
\hline $\mathrm{C}$ & 4.94834500 & -0.08500500 & 0.21697700 \\
\hline $\mathrm{H}$ & 5.49135000 & 1.97953900 & 0.51174500 \\
\hline $\mathrm{H}$ & 4.07124900 & -2.02251000 & -0.15835200 \\
\hline $\mathrm{C}$ & 6.33509800 & -0.62859300 & 0.45917200 \\
\hline $\mathrm{H}$ & 7.06350800 & 0.17883100 & 0.58498700 \\
\hline $\mathrm{H}$ & 6.36774900 & -1.25095200 & 1.36313300 \\
\hline $\mathrm{H}$ & 6.66911400 & -1.25595400 & -0.37669000 \\
\hline $\mathrm{N}$ & -0.55457400 & 1.15549400 & 0.92039800 \\
\hline $\mathrm{H}$ & -0.14795500 & 1.26645900 & 1.85022300 \\
\hline 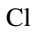 & -3.21241900 & -0.87733300 & -1.25069000 \\
\hline
\end{tabular}

Zero-point correction $=\quad 0.382192($ Hartree/Particle $)$

Thermal correction to Energy= $\quad 0.410117$

Thermal correction to Enthalpy= $\quad 0.411061$

Thermal correction to Gibbs Free Energy $=0.319105$

Sum of electronic and zero-point Energies $=\quad-1931.523936$

Sum of electronic and thermal Energies= $\quad-1931.496011$

Sum of electronic and thermal Enthalpies $=\quad-1931.495066$

Sum of electronic and thermal Free Energies $=\quad-1931.587023$

(U)B3LYP-D3/6-311++G(2d,p)-SDD/SMD//(U)B3LYP-D3/6-31+G(d)-LANL2DZ energy = -1933.9488788

$\begin{array}{cccc}{ }^{2} \text { INT8 } & & & \\ \text { C } & 0.11412500 & -2.97022800 & 0.10844900 \\ \text { C } & -0.42634700 & -2.77901400 & -1.28876700 \\ \text { H } & -0.61365300 & -3.51662600 & 0.71863900 \\ \text { H } & 1.06784100 & -3.51304300 & 0.07547800 \\ \text { H } & -1.24645900 & -2.04595000 & -1.27138100 \\ \text { H } & -0.83655400 & -3.73769800 & -1.64475100 \\ \text { O } & 0.62002800 & -2.34430200 & -2.14443900 \\ \text { O } & 0.34779500 & -1.67994000 & 0.70776200 \\ \text { C } & 0.15425100 & -2.01415800 & -3.44523100 \\ \text { H } & -0.57395700 & -1.19431200 & -3.40246100 \\ \text { H } & 1.02468800 & -1.69414000 & -4.02315000 \\ \text { H } & -0.30191700 & -2.88980500 & -3.93195200 \\ \text { C } & 1.00958400 & -1.77096500 & 1.96833900 \\ \text { H } & 1.96448100 & -2.29899300 & 1.85105000 \\ \text { H } & 1.20411600 & -0.74905100 & 2.30016200 \\ \text { H } & 0.37787200 & -2.28875400 & 2.70039200 \\ \text { C } & -4.49375700 & 2.60085200 & -0.16017900 \\ \text { C } & -3.20825400 & 1.78864600 & -0.34071000\end{array}$




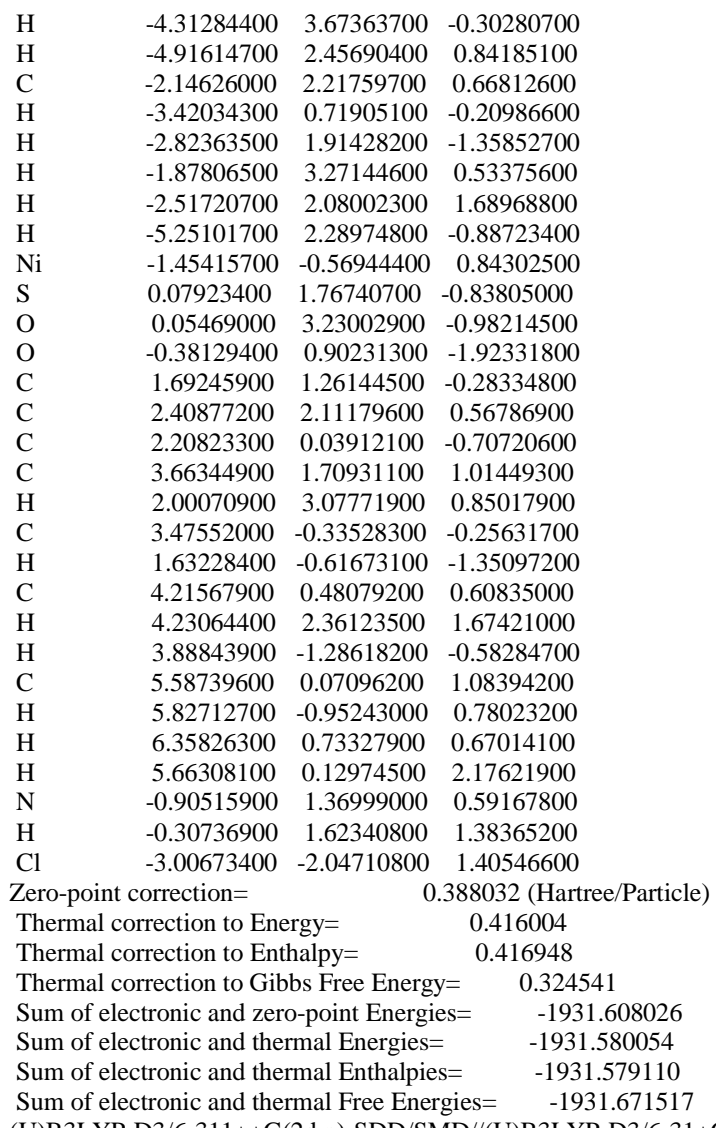

(U)B3LYP-D3/6-311++G(2d,p)-SDD/SMD//(U)B3LYP-D3/6-31+G(d)-LANL2DZ energy = -1934.0320982

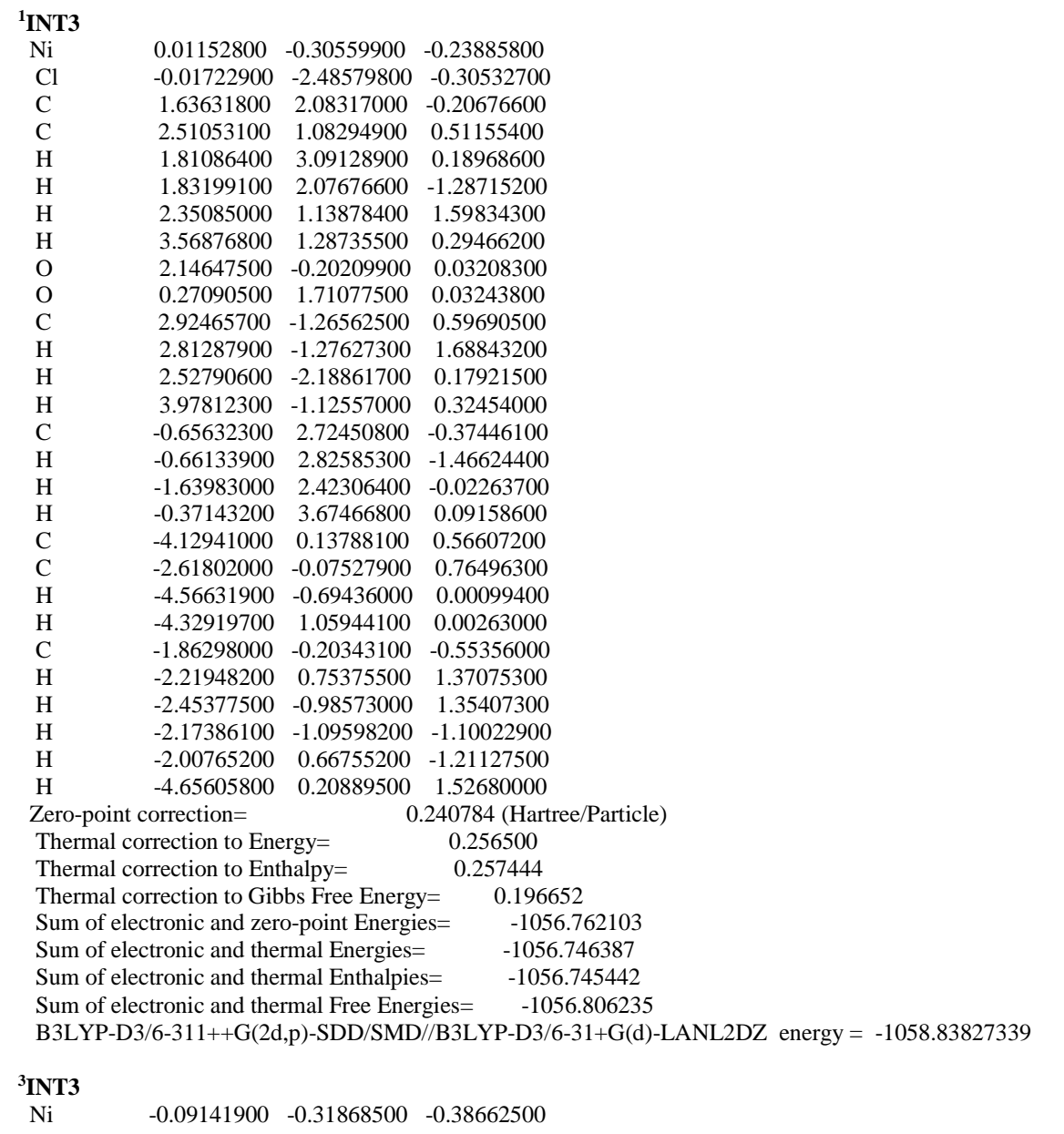




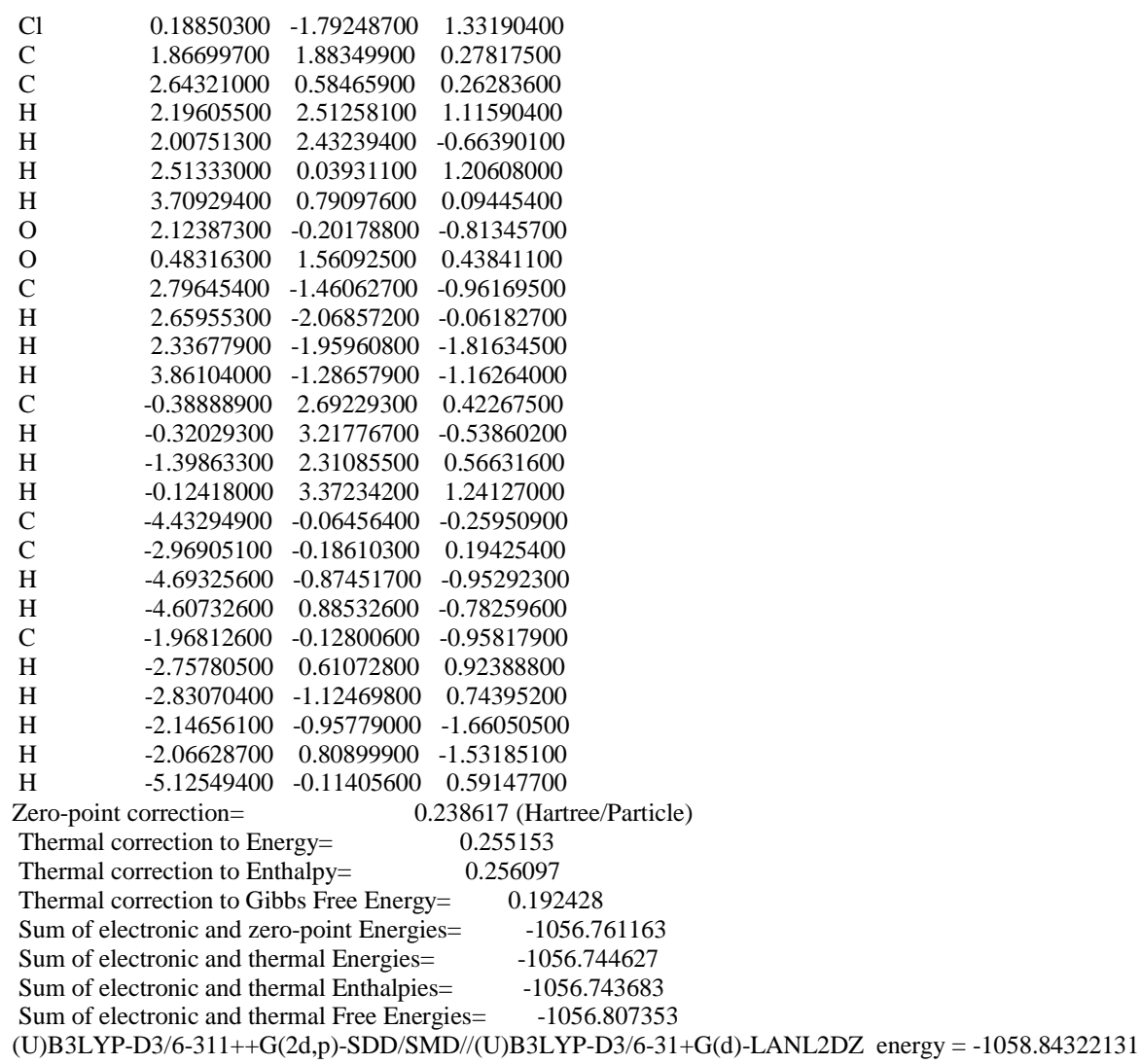

$\begin{array}{lccc}{ }^{1} \mathbf{I N T 4} & & & \\ \mathrm{C} & 1.26732900 & -2.15847600 & 0.92521300 \\ \mathrm{C} & 0.51125000 & -2.56599800 & -0.32060400 \\ \mathrm{H} & 1.72933500 & -3.06856700 & 1.34382100 \\ \mathrm{H} & 2.07248500 & -1.45264100 & 0.67433000 \\ \mathrm{H} & -0.31673000 & -3.24387200 & -0.08493000 \\ \mathrm{H} & 1.21633700 & -3.07151000 & -0.99539800 \\ \mathrm{O} & -0.01296600 & -1.41079800 & -0.99101500 \\ \mathrm{O} & 0.39174200 & -1.58042000 & 1.87684400 \\ \mathrm{C} & -0.29929500 & -1.65871200 & -2.37627900 \\ \mathrm{H} & -1.05374700 & -2.44384100 & -2.47488200 \\ \mathrm{H} & -0.68202400 & -0.72343000 & -2.78546100 \\ \mathrm{H} & 0.62864900 & -1.94511200 & -2.89021700 \\ \mathrm{C} & 1.00283000 & -1.41630800 & 3.14369900 \\ \mathrm{H} & 1.86890900 & -0.73777500 & 3.08472400 \\ \mathrm{H} & 0.25260400 & -0.98660200 & 3.81005800 \\ \mathrm{H} & 1.33614800 & -2.38380800 & 3.54942000 \\ \mathrm{C} & -5.11326500 & 1.98894300 & 0.01005400 \\ \mathrm{C} & -3.89330700 & 1.17223800 & -0.45392400 \\ \mathrm{H} & -4.84368800 & 2.66936500 & 0.82822000 \\ \mathrm{H} & -5.90871700 & 1.32742800 & 0.37463800 \\ \mathrm{C} & -3.31148500 & 0.31527800 & 0.66283200 \\ \mathrm{H} & -4.18808500 & 0.52333100 & -1.28794800 \\ \mathrm{H} & -3.13264300 & 1.86163300 & -0.84714800 \\ \mathrm{H} & -2.99904100 & 0.92692800 & 1.52287900 \\ \mathrm{H} & -4.01863200 & -0.43983300 & 1.01120600 \\ \mathrm{H} & -5.52328200 & 2.59282800 & -0.80980100 \\ \mathrm{Ni} & -1.76577300 & -0.58438200 & -0.02103300 \\ \mathrm{~S} & 0.37013100 & 1.97060700 & -0.66098000 \\ \mathrm{O} & 0.40394000 & 3.28964400 & -0.03108700 \\ \mathrm{O} & -0.19449700 & 1.72599600 & -1.97933300 \\ \mathrm{C} & 1.97687300 & 1.22387200 & -0.53214500 \\ \mathrm{C} & 2.75171000 & 1.47758700 & 0.60318700 \\ \mathrm{C} & 2.42895100 & 0.39542700 & -1.55785900 \\ \mathrm{C} & 3.99731100 & 0.86465900 & 0.71174200 \\ \mathrm{H} & 2.39493700 & 2.14928100 & 1.37672000 \\ \mathrm{C} & 3.68539200 & -0.19460300 & -1.43537000 \\ \mathrm{H} & 1.80744000 & 0.22190500 & -2.42712800 \\ \mathrm{C} & 4.48421300 & 0.02381800 & -0.30284500 \\ \mathrm{H} & 4.60695000 & 1.05130600 & 1.59192600 \\ \mathrm{H} & 4.05039600 & -0.83703400 & -2.23231700 \\ \mathrm{C} & 5.85164900 & -0.60116600 & -0.18835100 \\ \mathrm{H} & 6.62833200 & 0.11068500 & -0.49716300\end{array}$




$$
\begin{array}{lrrc}
\mathrm{H} & 6.07128100 & -0.89581200 & 0.84332700 \\
\mathrm{H} & 5.94284600 & -1.48596600 & -0.82589400 \\
\mathrm{~N} & -0.63509300 & 0.95277300 & 0.41499100 \\
\mathrm{Cl} & -2.92615000 & -2.38775300 & -0.48878400 \\
\mathrm{~N} & -0.46920700 & 1.53574700 & 2.72021800 \\
\mathrm{~N} & -0.52750900 & 1.27021900 & 1.61930300
\end{array}
$$

Zero-point correction $=\quad 0.382603$ (Hart

Thermal correction to Energy= $\quad 0.412578$

Thermal correction to Enthalpy= $\quad 0.413522$

Thermal correction to Gibbs Free Energy $=0.317778$

Sum of electronic and zero-point Energies $=\quad-2040.412655$

Sum of electronic and thermal Energies $=\quad-2040.382681$

Sum of electronic and thermal Enthalpies $=\quad-2040.381736$

Sum of electronic and thermal Free Energies $=\quad-2040.477480$

B3LYP-D3/6-311++G(2d,p)-SDD/SMD//B3LYP-D3/6-31+G(d)-LANL2DZ energy = -2042.85811277

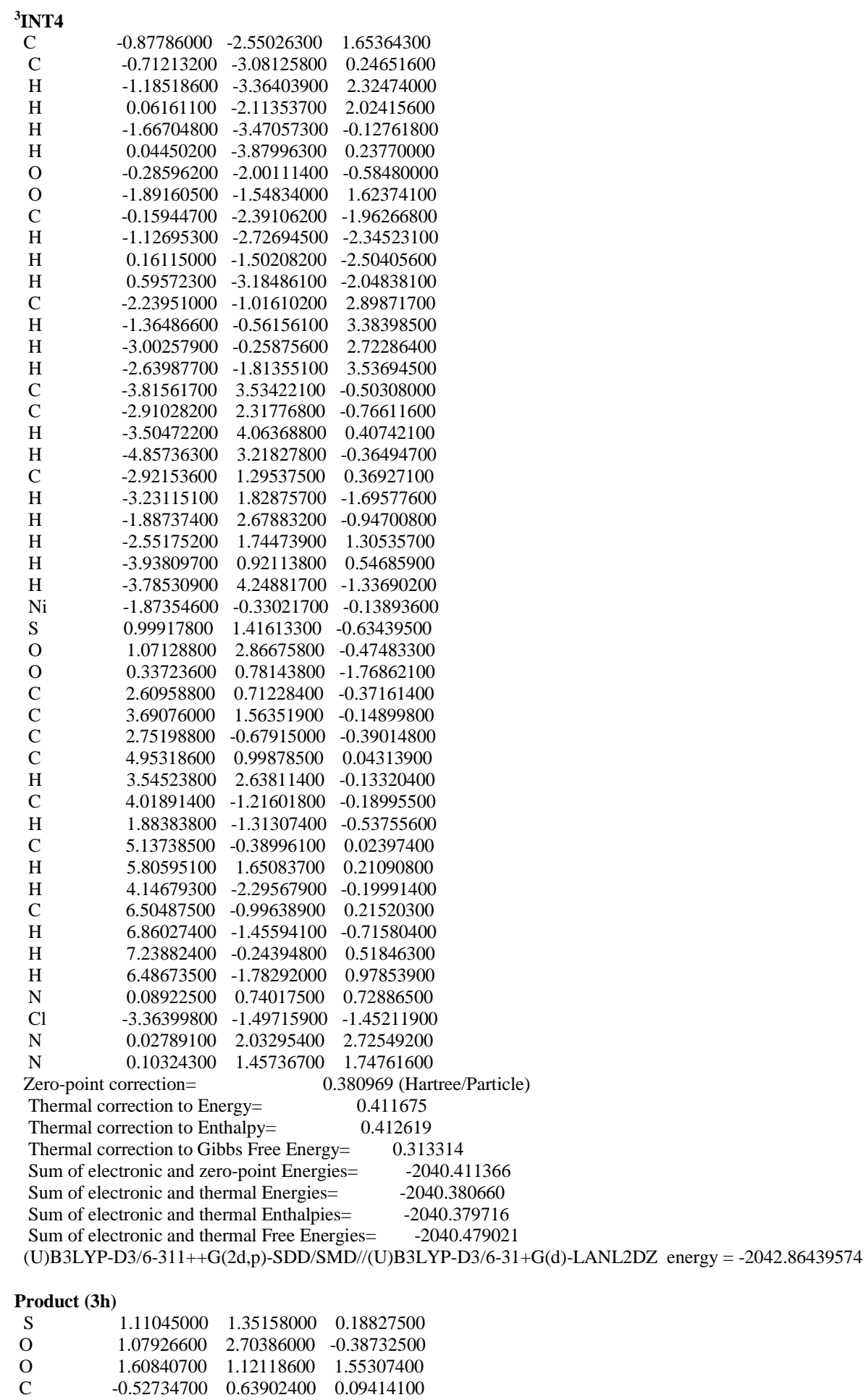




\begin{tabular}{|c|c|c|c|}
\hline $\mathrm{C}$ & -1.12117300 & $0.13953300 \quad 1.2$ & 973600 \\
\hline $\mathrm{C}$ & -1.20267800 & $0.63121000-1.1$ & 882800 \\
\hline $\mathrm{C}$ & -2.41305700 & $-0.38634300 \quad 1.1$ & 362400 \\
\hline $\mathrm{H}$ & -0.57571300 & $0.16113700 \quad 2.1$ & 706600 \\
\hline $\mathrm{C}$ & -2.48677100 & $0.10010900-1.1$ & 685500 \\
\hline $\mathrm{H}$ & -0.72376400 & $1.02918000-2.0$ & 730000 \\
\hline $\mathrm{C}$ & -3.11244100 & $-0.41607300-0.0$ & 860400 \\
\hline $\mathrm{H}$ & -2.88165700 & $-0.77848500 \quad 2.0$ & 262600 \\
\hline $\mathrm{H}$ & -3.01671300 & $0.08661300-2.1$ & 639100 \\
\hline $\mathrm{N}$ & 1.98752100 & $0.43587800-0.9$ & 486900 \\
\hline $\mathrm{C}$ & 2.48927200 & $-3.23149800 \quad 0.1$ & 819100 \\
\hline $\mathrm{C}$ & 1.73116700 & $\begin{array}{ll}-1.95337400 & -0.2\end{array}$ & 090000 \\
\hline $\mathrm{H}$ & 3.06361800 & $-3.09868600 \quad 1.0$ & 339700 \\
\hline $\mathrm{H}$ & 3.19236100 & $-3.52131400-0.6$ & 319700 \\
\hline $\mathrm{C}$ & 2.68357500 & $-0.77615200-0.4$ & 714400 \\
\hline $\mathrm{H}$ & 1.14764300 & $-2.10135000-1.1$ & 801700 \\
\hline $\mathrm{H}$ & 1.01480800 & $-1.71175100 \quad 0.5$ & 196500 \\
\hline $\mathrm{H}$ & 3.24222100 & $-0.57280200 \quad 0.4$ & 598200 \\
\hline $\mathrm{H}$ & 3.40891600 & $-1.02413800-1.2$ & 051200 \\
\hline $\mathrm{C}$ & -4.50990600 & $-0.97916300-0.1$ & 047900 \\
\hline $\mathrm{H}$ & -4.57449000 & $-1.77126600-0.8$ & 621600 \\
\hline $\mathrm{H}$ & -4.82868000 & $-1.39958800 \quad 0.8$ & 821100 \\
\hline $\mathrm{H}$ & -5.23090500 & $-0.20192700-0.4$ & 311300 \\
\hline $\mathrm{H}$ & 1.79848800 & $-4.06767200 \quad 0.2$ & 259000 \\
\hline $\mathrm{H}$ & 2.59879500 & $1.09162900-1.4$ & 869700 \\
\hline \multicolumn{2}{|c|}{ Zero-point correction= } & 0.240 & 582 (Hartree/Particle) \\
\hline \multicolumn{3}{|c|}{ Thermal correction to Energy= } & 54783 \\
\hline \multicolumn{3}{|c|}{ Thermal correction to Enthalpy= } & 255727 \\
\hline \multicolumn{3}{|c|}{ Thermal correction to Gibbs Free Energy= } & 0.197384 \\
\hline \multicolumn{3}{|c|}{ Sum of electronic and zero-point Energies= } & -993.268235 \\
\hline \multicolumn{3}{|c|}{ Sum of electronic and thermal Energies= } & -993.254034 \\
\hline \multicolumn{3}{|c|}{ Sum of electronic and thermal Enthalpies= } & -993.253090 \\
\hline \multicolumn{3}{|c|}{ Sum of electronic and thermal Free Energies } & -993.311433 \\
\hline
\end{tabular}

B3LYP-D3/6-311++G(2d,p)/SMD//B3LYP-D3/6-31+G(d) energy = -993.745463

$\begin{array}{cccc}{ }^{2} \text { INT5 } & & & \\ \mathrm{C} & 1.62611800 & 2.94045200 & 0.62497300 \\ \mathrm{C} & 0.99267100 & 3.14961500 & -0.73284200 \\ \mathrm{H} & 2.07954300 & 3.87587400 & 0.97865900 \\ \mathrm{H} & 0.88507900 & 2.59549300 & 1.35811400 \\ \mathrm{H} & 1.74534300 & 3.48216300 & -1.46186000 \\ \mathrm{H} & 0.19486300 & 3.90305800 & -0.67136400 \\ \mathrm{O} & 0.44970400 & 1.89541400 & -1.13884500 \\ \mathrm{O} & 2.64345300 & 1.94380700 & 0.46859300 \\ \mathrm{C} & -0.06853000 & 1.87131100 & -2.47178700 \\ \mathrm{H} & 0.72233500 & 2.11452400 & -3.19330200 \\ \mathrm{H} & -0.43177800 & 0.85719000 & -2.63550000 \\ \mathrm{H} & -0.89115300 & 2.59156100 & -2.56566200 \\ \mathrm{C} & 3.35560800 & 1.68523500 & 1.69201500 \\ \mathrm{H} & 2.67260700 & 1.28620000 & 2.44991900 \\ \mathrm{H} & 4.12511700 & 0.95308200 & 1.45371800 \\ \mathrm{H} & 3.81789400 & 2.61621000 & 2.04161500 \\ \mathrm{C} & 4.19381500 & -3.25663700 & -0.83187500 \\ \mathrm{C} & 2.96393200 & -2.33602700 & -0.93309700 \\ \mathrm{H} & 4.41466900 & -3.49783500 & 0.21572900 \\ \mathrm{H} & 5.08453700 & -2.77613000 & -1.25761400 \\ \mathrm{C} & 3.15629500 & -1.02134900 & -0.19038700 \\ \mathrm{H} & 2.74546000 & -2.14523400 & -1.99297600 \\ \mathrm{H} & 2.08903800 & -2.86176800 & -0.53505300 \\ \mathrm{H} & 3.26847300 & -1.18428100 & 0.88907800 \\ \mathrm{H} & 4.03473400 & -0.46732300 & -0.56358300 \\ \mathrm{H} & 4.02946500 & -4.20063600 & -1.36783400 \\ \mathrm{Ni} & 1.69003500 & 0.23736800 & -0.41573300 \\ \mathrm{~S} & -0.57638200 & -1.39499700 & 0.09290800 \\ \mathrm{O} & -0.62760800 & -2.81091400 & 0.47485800 \\ \mathrm{O} & 0.08154900 & -1.04571200 & -1.22098700 \\ \mathrm{C} & -2.25957600 & -0.79037900 & 0.03681300 \\ \mathrm{C} & -3.31649000 & -1.69147200 & 0.13601700 \\ \mathrm{C} & -2.48781400 & 0.58130000 & -0.10690300 \\ \mathrm{C} & -4.62565000 & -1.20721600 & 0.07707600 \\ \mathrm{H} & -3.11018600 & -2.74878100 & 0.26204600 \\ \mathrm{C} & -3.79847900 & 1.04514700 & -0.16190300 \\ \mathrm{H} & -1.64611800 & 1.26268700 & -0.16620400 \\ \mathrm{H} & -4.88721300 & 0.16051200 & -0.07106700 \\ \mathrm{H} & -5.45509200 & -1.90578000 & 0.15259900 \\ \mathrm{H} & -3.98521000 & 2.11143600 & -0.26988600 \\ & -6.30214900 & 0.68425600 & -0.11033000 \\ \mathrm{H} & -6.44739700 & 1.37772400 & -0.94703300 \\ \mathrm{H} & -7.02681700 & -0.12929200 & -0.21422600 \\ \mathrm{H} & & & \\ \mathrm{H} & & 1.23065400 & 0.81054300\end{array}$




$\begin{array}{lrrr}\mathrm{N} & 0.29457300 & -0.38536300 & 1.06710900 \\ \mathrm{~N} & -0.12280300 & -0.37141100 & 2.39206700 \\ \mathrm{~N} & 0.38504000 & 0.26872000 & 3.25152400 \\ \text { Zero-point correction= } & 0.378169 & \text { (Hartree/Particle) } \\ \text { Thermal correction to Energy= } & 0.406697 \\ \text { Thermal correction to Enthalpy= } & 0.407641 \\ \text { Thermal correction to Gibbs Free Energy= } & 0.314973 \\ \text { Sum of electronic and zero-point Energies= } & -1580.165361 \\ \text { Sum of electronic and thermal Energies }= & -1580.136833 \\ \text { Sum of electronic and thermal Enthalpies= } & -1580.135889 \\ \text { Sum of electronic and thermal Free Energies= } & -1580.228557\end{array}$

(U)B3LYP-D3/6-311++G(2d,p)-SDD/SMD//(U)B3LYP-D3/6-31+G(d)-LANL2DZ energy = -1582.56569357

\begin{tabular}{|c|c|c|c|}
\hline \multicolumn{4}{|c|}{ 'INT5', } \\
\hline $\mathrm{C}$ & 1.45664100 & 2.96858500 & 0.74289800 \\
\hline $\mathrm{C}$ & 0.92381300 & 3.21525500 & -0.65355100 \\
\hline $\mathrm{H}$ & 1.85842700 & 3.90254800 & 1.15818300 \\
\hline $\mathrm{H}$ & 0.67191500 & 2.58288400 & 1.40682300 \\
\hline $\mathrm{H}$ & 1.71622500 & 3.62186000 & -1.29838700 \\
\hline $\mathrm{H}$ & 0.08745600 & 3.92848600 & -0.62171300 \\
\hline $\mathrm{O}$ & 0.48024600 & 1.96340600 & -1.16763900 \\
\hline $\mathrm{O}$ & 2.50354100 & 2.00164300 & 0.62664800 \\
\hline $\mathrm{C}$ & 0.04970500 & 1.99599500 & -2.52927800 \\
\hline $\mathrm{H}$ & 0.87302700 & 2.30827500 & -3.18531200 \\
\hline $\mathrm{H}$ & -0.26450400 & 0.98068500 & -2.77125600 \\
\hline $\mathrm{H}$ & -0.79367600 & 2.69006700 & -2.64165200 \\
\hline $\mathrm{C}$ & 3.24497000 & 1.80428000 & 1.84201800 \\
\hline $\mathrm{H}$ & 2.58796500 & 1.42275900 & 2.63049000 \\
\hline $\mathrm{H}$ & 4.01739400 & 1.07129200 & 1.61274600 \\
\hline $\mathrm{H}$ & 3.70417900 & 2.75392900 & 2.14270300 \\
\hline $\mathrm{C}$ & 4.27983900 & -3.23166900 & -0.94892300 \\
\hline $\mathrm{C}$ & 3.02880900 & -2.33703200 & -0.92055100 \\
\hline $\mathrm{H}$ & 4.68508700 & -3.36716400 & 0.06237600 \\
\hline $\mathrm{H}$ & 5.07065800 & -2.78273200 & -1.56462800 \\
\hline $\mathrm{C}$ & 3.29447200 & -0.94526100 & -0.35073200 \\
\hline $\mathrm{H}$ & 2.62354400 & -2.25588900 & -1.93869300 \\
\hline $\mathrm{H}$ & 2.24812700 & -2.83527600 & -0.33137500 \\
\hline $\mathrm{H}$ & 3.65540500 & -1.01349700 & 0.68587400 \\
\hline $\mathrm{H}$ & 4.06471000 & -0.41475200 & -0.93722900 \\
\hline $\mathrm{H}$ & 4.05558200 & -4.22595300 & -1.35817700 \\
\hline $\mathrm{Ni}$ & 1.69295600 & 0.22402800 & -0.33498700 \\
\hline$S$ & -0.61302300 & -1.37790600 & -0.00628100 \\
\hline $\mathrm{O}$ & -0.62577900 & -2.79357400 & 0.37081300 \\
\hline $\mathrm{O}$ & 0.00988500 & -0.97464500 & -1.31305900 \\
\hline $\mathrm{C}$ & -2.30297100 & -0.79619100 & -0.01015400 \\
\hline $\mathrm{C}$ & -3.33880200 & -1.69024000 & 0.24441900 \\
\hline $\mathrm{C}$ & -2.55492100 & 0.55745500 & -0.25594000 \\
\hline $\mathrm{C}$ & -4.65454300 & -1.21854500 & 0.23978600 \\
\hline $\mathrm{H}$ & -3.11240000 & -2.73196800 & 0.44417500 \\
\hline $\mathrm{C}$ & -3.87064800 & 1.00805000 & -0.25556500 \\
\hline $\mathrm{H}$ & -1.72665700 & 1.23490900 & -0.43404500 \\
\hline $\mathrm{C}$ & -4.94062500 & 0.12890000 & -0.00897500 \\
\hline $\mathrm{H}$ & -5.46857500 & -1.91137600 & 0.43665900 \\
\hline $\mathrm{H}$ & -4.07706700 & 2.05933100 & -0.44443100 \\
\hline $\mathrm{C}$ & -6.36098200 & 0.63910100 & -0.00959600 \\
\hline $\mathrm{H}$ & -6.61356200 & 1.10585900 & -0.96968800 \\
\hline $\mathrm{H}$ & -7.07618900 & -0.16933400 & 0.17036600 \\
\hline $\mathrm{H}$ & -6.50839700 & 1.39817600 & 0.76884000 \\
\hline $\mathrm{N}$ & 0.23951500 & -0.35910600 & 0.98914300 \\
\hline $\mathrm{N}$ & 0.12843500 & -0.66762800 & 2.33820300 \\
\hline $\mathrm{N}$ & 0.72565600 & -0.07986000 & 3.18240100 \\
\hline
\end{tabular}

Zero-point correction $=\quad 0.377894($ Hartree/Particle $)$

Thermal correction to Energy $=\quad 0.406565$

Thermal correction to Enthalpy= $\quad 0.407510$

Thermal correction to Gibbs Free Energy $=0.313720$

Sum of electronic and zero-point Energies $=\quad-1580.175137$

Sum of electronic and thermal Energies $=\quad-1580.146466$

Sum of electronic and thermal Enthalpies $=\quad-1580.145522$

Sum of electronic and thermal Free Energies $=\quad-1580.239311$

(U)B3LYP-D3/6-311++G(2d,p)-SDD/SMD//(U)B3LYP-D3/6-31+G(d)-LANL2DZ energy = -1582.57864622

$\begin{array}{cccc}{ }^{\mathbf{4}} \mathbf{T S 1} & & & \\ \mathrm{C} & 1.63371600 & 2.72793900 & 1.23343700 \\ \mathrm{C} & 1.00309000 & 3.35291900 & 0.00577200 \\ \mathrm{H} & 2.13283300 & 3.50124200 & 1.83283800 \\ \mathrm{H} & 0.87966000 & 2.21732900 & 1.84669200 \\ \mathrm{H} & 1.76362900 & 3.87678000 & -0.59131800 \\ \mathrm{H} & 0.22437700 & 4.07040100 & 0.30228800 \\ \mathrm{O} & 0.43124800 & 2.29798100 & -0.76031300 \\ \mathrm{O} & 2.59596100 & 1.77700500 & 0.77115500\end{array}$




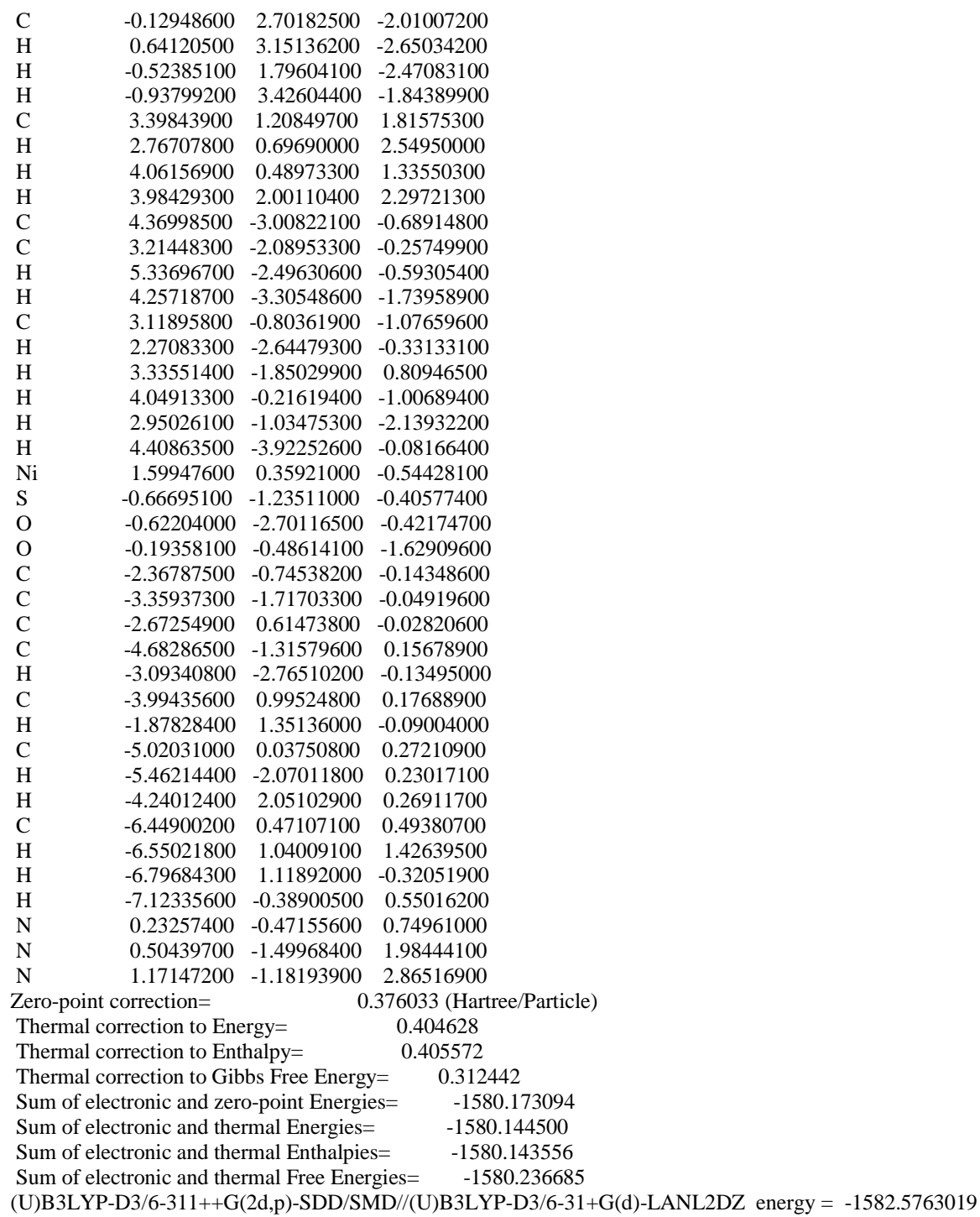

$\begin{array}{cccc}{ }^{4} \mathbf{I N T 6} & & & \\ \mathrm{C} & 2.43912300 & -2.90624200 & -0.61772300 \\ \mathrm{C} & 2.88092000 & -2.53146800 & 0.78273700 \\ \mathrm{H} & 3.17511600 & -3.57768500 & -1.08167500 \\ \mathrm{H} & 1.46228500 & -3.41057000 & -0.58842700 \\ \mathrm{H} & 3.85857700 & -2.03118300 & 0.76181700 \\ \mathrm{H} & 2.94291500 & -3.42621300 & 1.41650500 \\ \mathrm{O} & 1.89591400 & -1.63627100 & 1.30312100 \\ \mathrm{O} & 2.33745400 & -1.69832700 & -1.36576300 \\ \mathrm{C} & 2.16145400 & -1.15272200 & 2.62615200 \\ \mathrm{H} & 3.12417300 & -0.62828300 & 2.65238700 \\ \mathrm{H} & 1.35333100 & -0.46028900 & 2.86290500 \\ \mathrm{H} & 2.16835000 & -1.99298700 & 3.33081800 \\ \mathrm{C} & 1.79957900 & -1.86328500 & -2.68253600 \\ \mathrm{H} & 0.79189700 & -2.29186600 & -2.63316400 \\ \mathrm{H} & 1.74117600 & -0.86665300 & -3.11787400 \\ \mathrm{H} & 2.46367600 & -2.50629600 & -3.27355400 \\ \mathrm{C} & 3.85240900 & 3.53656500 & 0.27137300 \\ \mathrm{C} & 2.65983100 & 2.56148800 & 0.28115400 \\ \mathrm{H} & 4.27680200 & 3.62535500 & -0.73700500 \\ \mathrm{H} & 4.65286200 & 3.18880400 & 0.93804800 \\ \mathrm{C} & 3.02750800 & 1.16090900 & -0.17400800 \\ \mathrm{H} & 2.23231000 & 2.52769500 & 1.29239600 \\ \mathrm{H} & 1.86469800 & 2.95952300 & -0.36125000 \\ \mathrm{H} & 3.38798200 & 1.12807600 & -1.20967700 \\ \mathrm{H} & 3.77245600 & 0.68025100 & 0.48171400 \\ \mathrm{H} & 3.54971400 & 4.53967400 & 0.60026100 \\ \mathrm{Ni} & 1.44220800 & -0.11922300 & -0.13531400 \\ \mathrm{~S} & -0.84362500 & 1.19361300 & -0.14571300 \\ \mathrm{O} & -0.95912200 & 2.63637700 & -0.42768700 \\ \mathrm{O} & -0.14593700 & 0.77601900 & 1.14769800\end{array}$




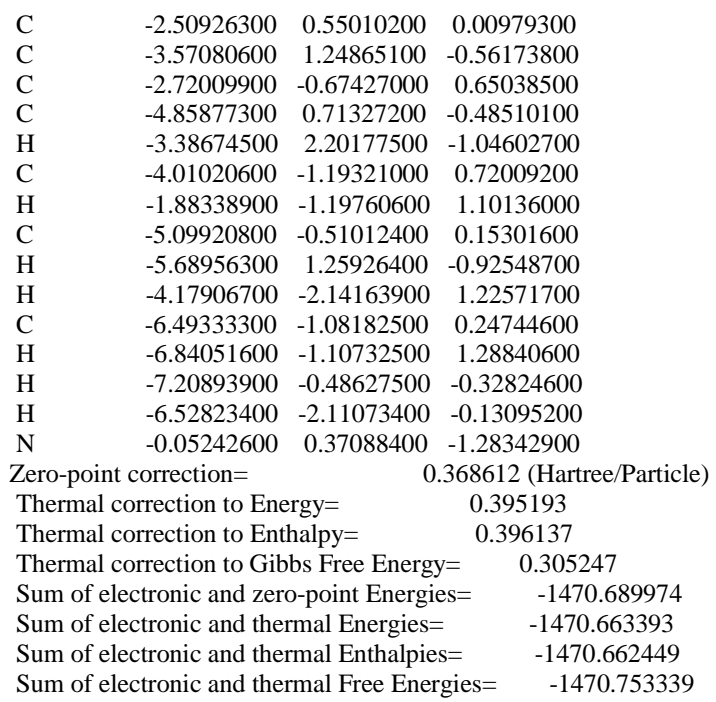

(U)B3LYP-D3/6-311++G(2d,p)-SDD/SMD//(U)B3LYP-D3/6-31+G(d)-LANL2DZ energy = -1473.06085

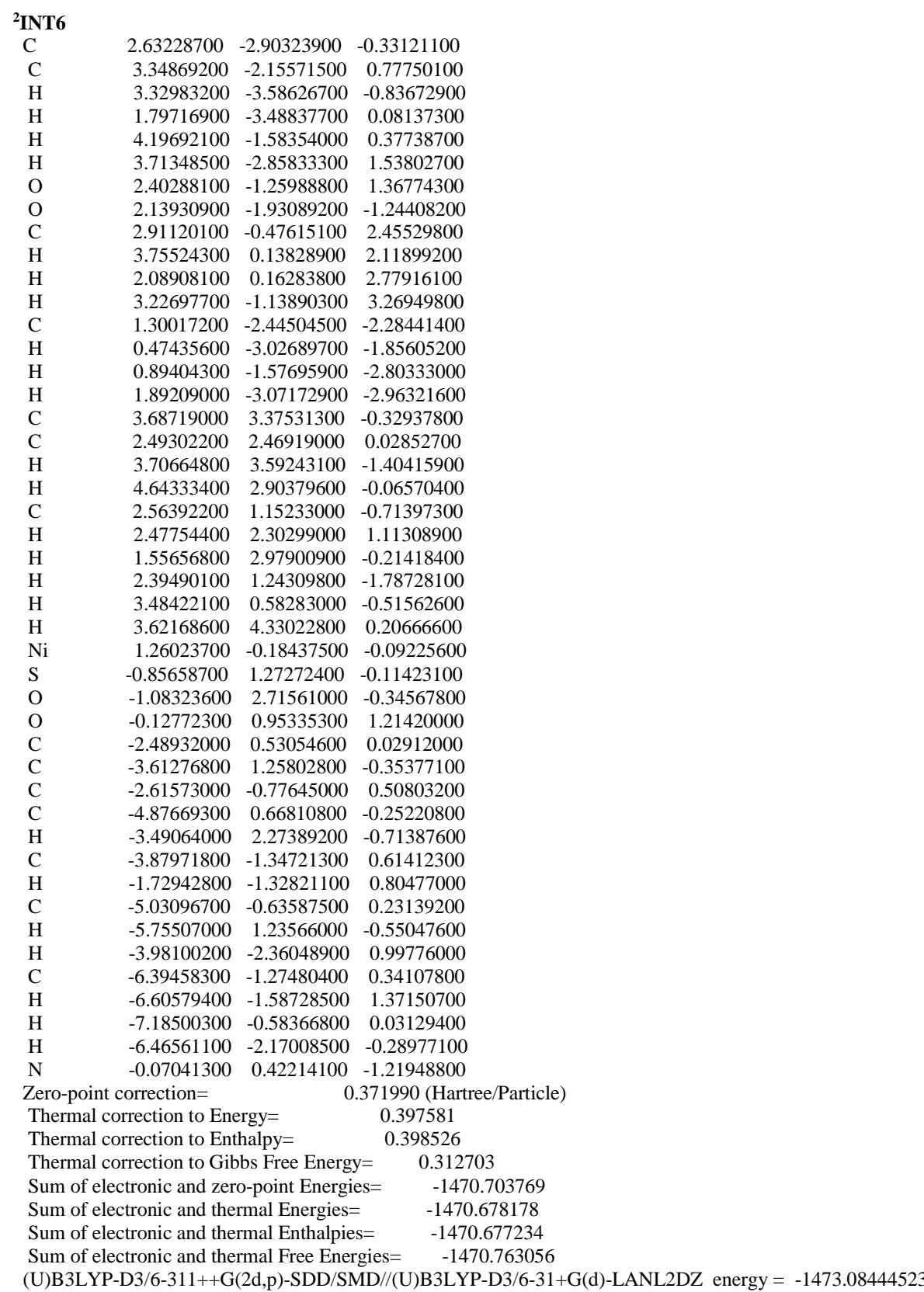




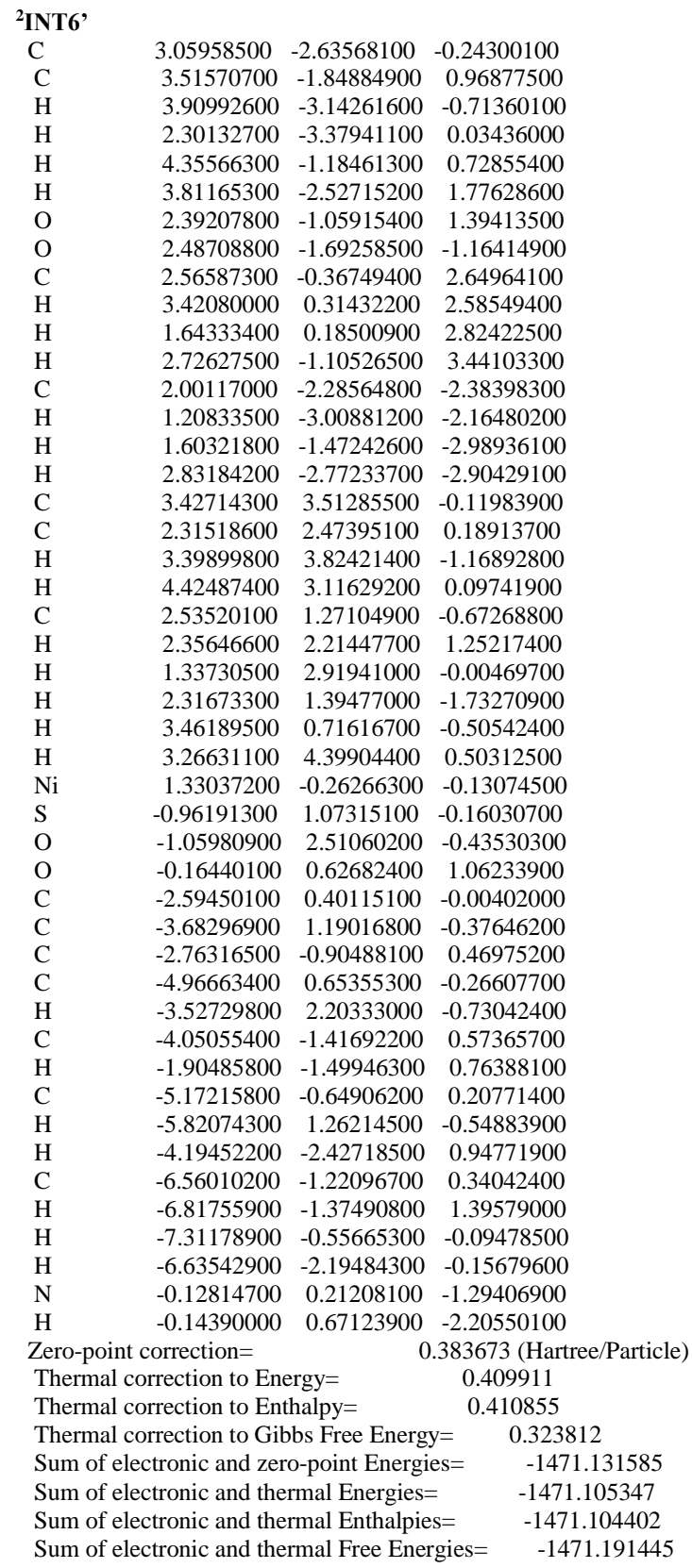

(U)B3LYP-D3/6-311++G(2d,p)-SDD/SMD//(U)B3LYP-D3/6-31+G(d)-LANL2DZ energy = -1473.57313922

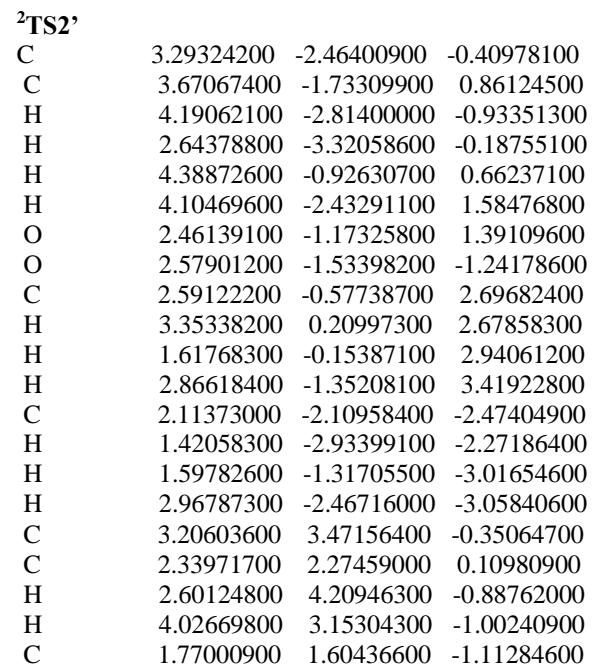




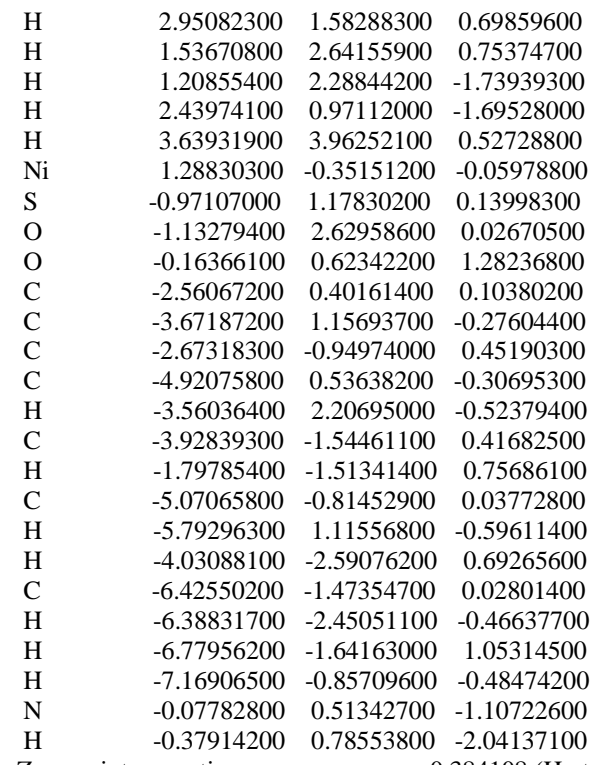

$\begin{array}{ll}\text { Zero-point correction }= & 0.384108 \text { (Hart }\end{array}$

Thermal correction to Energy= $\quad 0.409746$

Thermal correction to Enthalpy= $\quad 0.410690$

Thermal correction to Gibbs Free Energy $=\quad 0.324848$

Sum of electronic and zero-point Energies $=\quad-1471.113114$

Sum of electronic and thermal Energies $=\quad-1471.087476$

Sum of electronic and thermal Enthalpies $=\quad-1471.086532$

Sum of electronic and thermal Free Energies $=\quad-1471.172374$

(U)B3LYP-D3/6-311++G(2d,p)-SDD/SMD//(U)B3LYP-D3/6-31+G(d)-LANL2DZ energy = -1473.54572609

\section{${ }^{2}$ INT3-Bz}

$\begin{array}{llll}\text { C } & -2.85968700 & 1.44749600 & 1.03656700\end{array}$

$\begin{array}{llll}\mathrm{C} & -2.81366200 & 0.18738200 & 1.87656100\end{array}$

$\begin{array}{llll}\mathrm{H} & -3.81625000 & 1.96387600 & 1.19078300\end{array}$

$\begin{array}{llll}\mathrm{H} & -2.03296400 & 2.12739600 & 1.28125600\end{array}$

$\begin{array}{llll}\mathrm{H} & -3.63318800 & -0.48564300 & 1.59563700\end{array}$

$\begin{array}{llll}\mathrm{H} & -2.87640000 & 0.43025700 & 2.94484800\end{array}$

$\begin{array}{llll}\mathrm{O} & -1.56077600 & -0.46057700 & 1.61271800\end{array}$

$\begin{array}{lllll}\text { O } & -2.75697300 & 1.02641200 & -0.32466500\end{array}$

$\begin{array}{llll}\text { C } & -1.45199000 & -1.77271500 & 2.19579900\end{array}$

$\mathrm{H} \quad-2.23628800 \quad-2.42409400 \quad 1.79741000$

$\mathrm{H} \quad-0.47266500-2.15245100 \quad 1.90581000$

$\begin{array}{llll}\mathrm{H} & -1.52496500 & -1.68607200 & 3.28593000\end{array}$

$\begin{array}{llll}\mathrm{C} & -2.91984600 & 2.06355100 & -1.29903700\end{array}$

$\mathrm{H} \quad-2.13777500 \quad 2.82170700 \quad-1.18731400$

$\mathrm{H} \quad-2.84761300 \quad 1.57836000 \quad-2.27355100$

$\mathrm{H} \quad-3.91402000 \quad 2.51232200 \quad-1.18632500$

$\mathrm{Ni} \quad-1.19957600 \quad-0.42661700 \quad-0.44705500$

$\begin{array}{llll}\mathrm{Cl} & -2.56430700 & -2.02452300 & -1.25137000\end{array}$

$\begin{array}{llll}\mathrm{N} & 0.41360600 & 0.83693900 & -0.31946700\end{array}$

$\begin{array}{lll}0.87903500 & 2.14705300 & -0.36547700\end{array}$

$\begin{array}{lll}0.19556900 & 3.11287100 & -0.38414200\end{array}$

$1.30021300-0.17850100-0.23008000$

$0.72355200-1.31135300-0.28560700$

$\begin{array}{lll}2.77362300 & -0.10585100 & -0.08055200\end{array}$

$\begin{array}{lll}3.45133400 & 0.99166600 & 0.47531700\end{array}$

$3.50501400-1.23387500-0.49195500$

$\begin{array}{lll}4.84001600 & 0.95840700 & 0.60763800\end{array}$

$\begin{array}{lll}2.90107900 & 1.86116700 & 0.81046300\end{array}$

$\begin{array}{lll}4.89277200 & -1.25422200 & -0.37253100\end{array}$

$2.96838400 \quad-2.08133600-0.90460600$

$\begin{array}{lll}5.56342100 & -0.15758400 & 0.17852200\end{array}$

$\begin{array}{llll}5.35674800 & 1.80770800 & 1.04546400\end{array}$

$5.45058400 \quad-2.12513400-0.70480600$

$\begin{array}{lrrr}\mathrm{H} & 6.64555500 & -0.17433300 & 0.27637700 \\ \text { Zero-point correction= } & 0.260116 \text { (Hartree/Particle) }\end{array}$

Thermal correction to Energy= $\quad 0.281396$

Thermal correction to Enthalpy= $\quad 0.282340$

Thermal correction to Gibbs Free Energy $=0.207164$

Sum of electronic and zero-point Energies $=\quad-1447.460789$

Sum of electronic and thermal Energies $=\quad-1447.439508$

Sum of electronic and thermal Enthalpies $=\quad-1447.438564$

Sum of electronic and thermal Free Energies $=\quad-1447.513740$

(U)B3LYP-D3/6-311++G(2d,p)-SDD/SMD//(U)B3LYP-D3/6-31+G(d)-LANL2DZ energy = -1449.6703225 


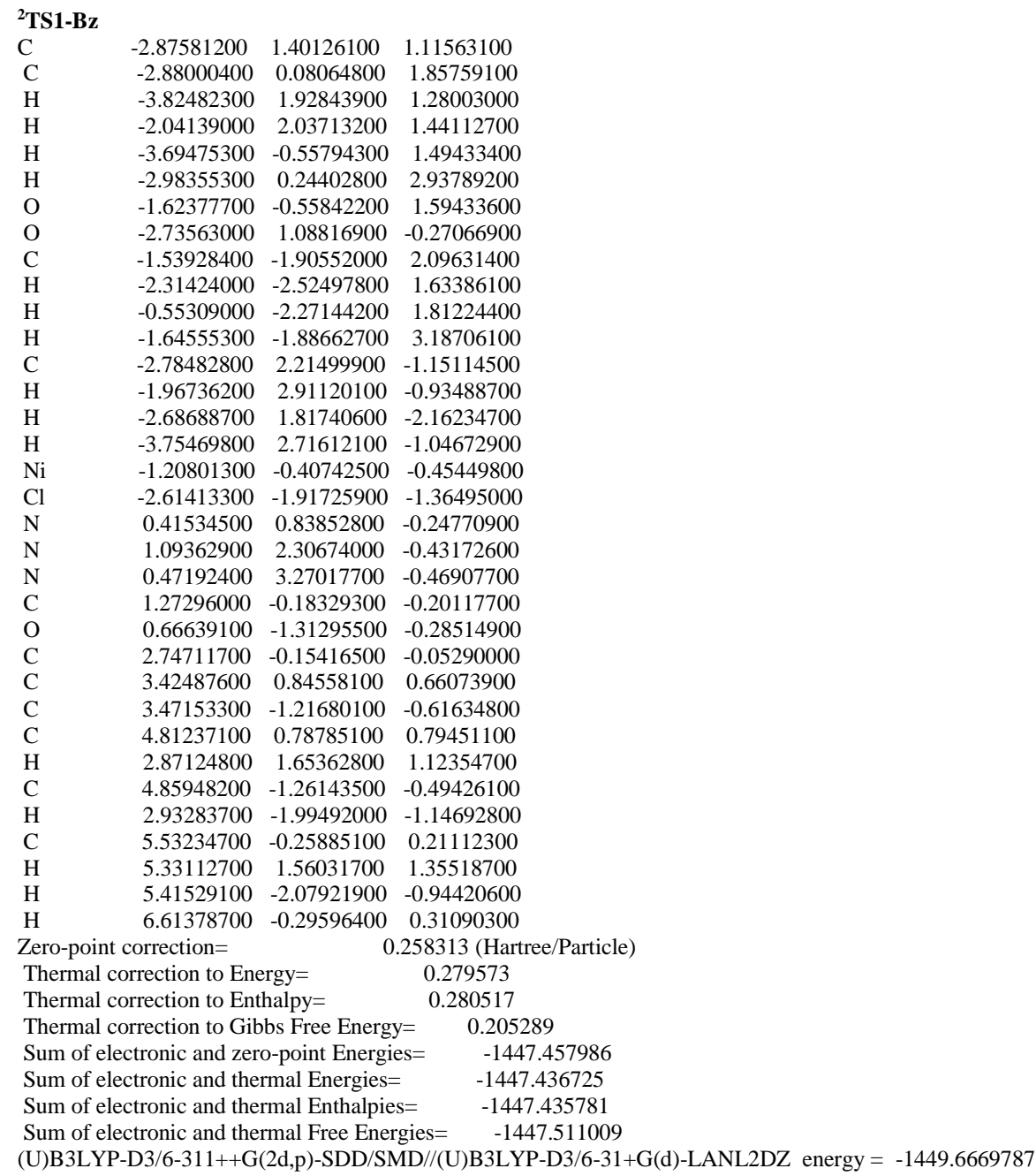

\section{${ }^{2}$ INT4-Bz}

$\begin{array}{lrrr}\text { C } & -2.70615300 & 1.66816000 & 1.02915400 \\ \mathrm{C} & -2.86621400 & 0.33364700 & 1.72622500 \\ \mathrm{H} & -3.61211200 & 2.27583700 & 1.14953000 \\ \mathrm{H} & -1.83816600 & 2.21168700 & 1.42512400 \\ \mathrm{H} & -3.69159100 & -0.23783900 & 1.28417400 \\ \mathrm{H} & -3.03941100 & 0.47877000 & 2.80021000 \\ \mathrm{O} & -1.63733800 & -0.37957700 & 1.53627700 \\ \mathrm{O} & -2.50504000 & 1.38915800 & -0.36118900 \\ \mathrm{C} & -1.66270500 & -1.73447800 & 2.02269400 \\ \mathrm{H} & -2.44946000 & -2.29941800 & 1.51363500 \\ \mathrm{H} & -0.68751200 & -2.15720300 & 1.78275200 \\ \mathrm{H} & -1.82094100 & -1.71889300 & 3.10724400 \\ \mathrm{C} & -2.21573700 & 2.54761100 & -1.15164600 \\ \mathrm{H} & -1.25851000 & 2.98550300 & -0.84870800 \\ \mathrm{H} & -2.16208700 & 2.20710400 & -2.18668200 \\ \mathrm{H} & -3.03002100 & 3.27346700 & -1.04257400 \\ \mathrm{Ni} & -1.11472700 & -0.21069500 & -0.47730700 \\ \mathrm{Cl} & -2.62006100 & -1.63808700 & -1.38379400 \\ \mathrm{~N} & 0.47317000 & 0.99231700 & -0.23426600 \\ \mathrm{C} & 1.29920000 & -0.01265700 & -0.21231800 \\ \mathrm{O} & 0.66227200 & -1.15947600 & -0.32389400 \\ \mathrm{C} & 2.76529000 & 0.04980100 & -0.07695200 \\ \mathrm{C} & 3.40456700 & 1.27760100 & 0.14711700 \\ \mathrm{C} & 3.51687700 & -1.12788800 & -0.17527200 \\ \mathrm{C} & 4.79161900 & 1.32497100 & 0.27339300 \\ \mathrm{H} & 2.80893100 & 2.18283600 & 0.22413100 \\ \mathrm{C} & 4.90575500 & -1.07610700 & -0.04765000 \\ \mathrm{H} & 3.00286200 & -2.06705300 & -0.35371900 \\ \mathrm{C} & 5.54352000 & 0.14799800 & 0.17625000 \\ \mathrm{H} & 5.28837000 & 2.27529400 & 0.44835900 \\ \mathrm{H} & 5.49051600 & -1.98848800 & -0.12497700 \\ \mathrm{H} & 6.62507500 & 0.18633800 & 0.27493700 \\ \mathrm{Z} & & & 0.251029(401\end{array}$




$\begin{array}{lc}\text { Thermal correction to Energy= } & 0.270114 \\ \text { Thermal correction to Enthalpy= } & 0.271058 \\ \text { Thermal correction to Gibbs Free Energy= } & 0.201206 \\ \text { Sum of electronic and zero-point Energies }= & -1337.972766 \\ \text { Sum of electronic and thermal Energies }= & -1337.953682 \\ \text { Sum of electronic and thermal Enthalpies }= & -1337.952737 \\ \text { Sum of electronic and thermal Free Energies }= & -1338.022589\end{array}$

Sum of electronic and thermal Free Energies=

(U)B3LYP-D3/6-311++G(2d,p)-SDD/SMD//(U)B3LYP-D3/6-31+G(d)-LANL2DZ energy = -1340.1475589
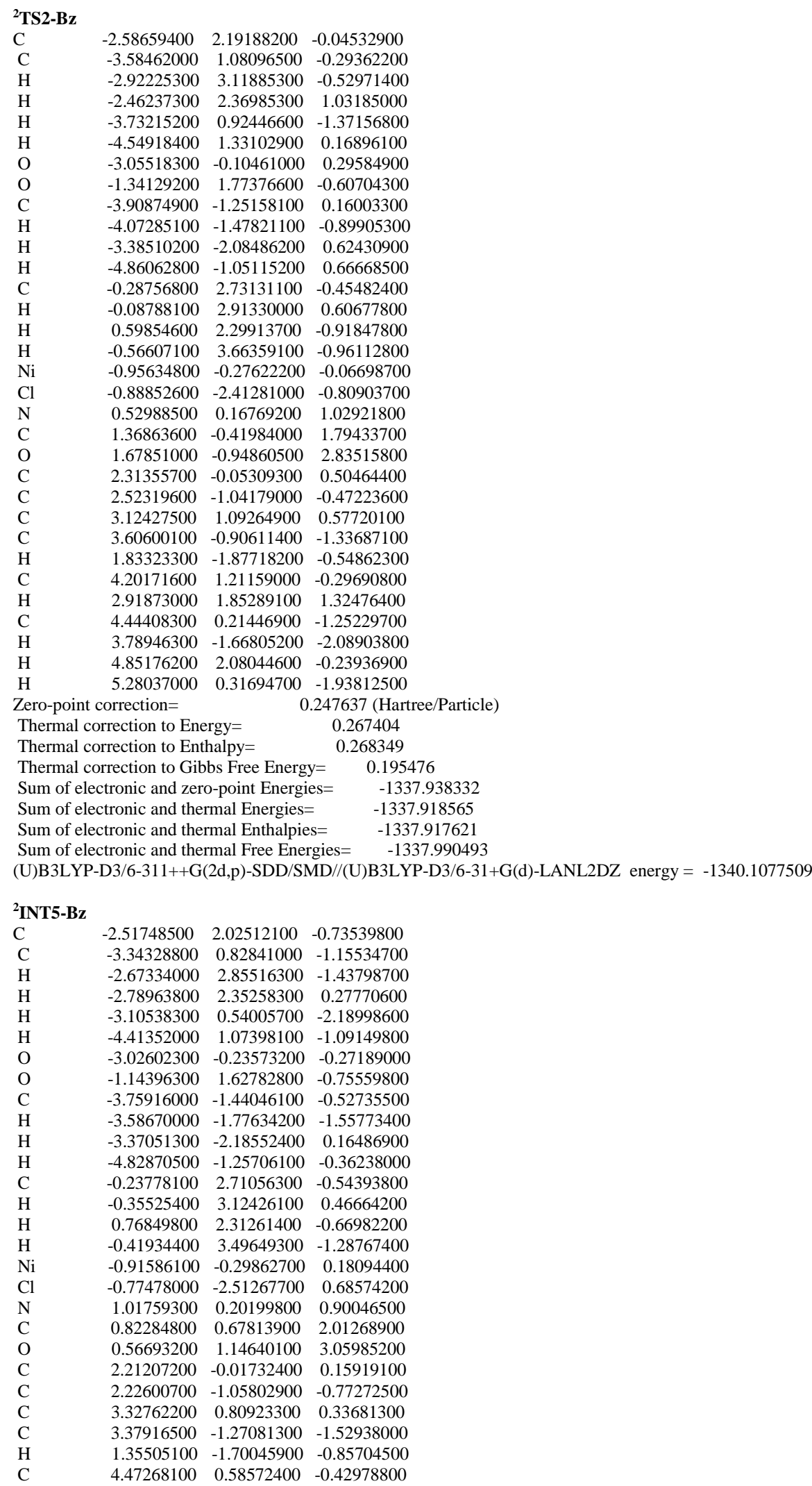

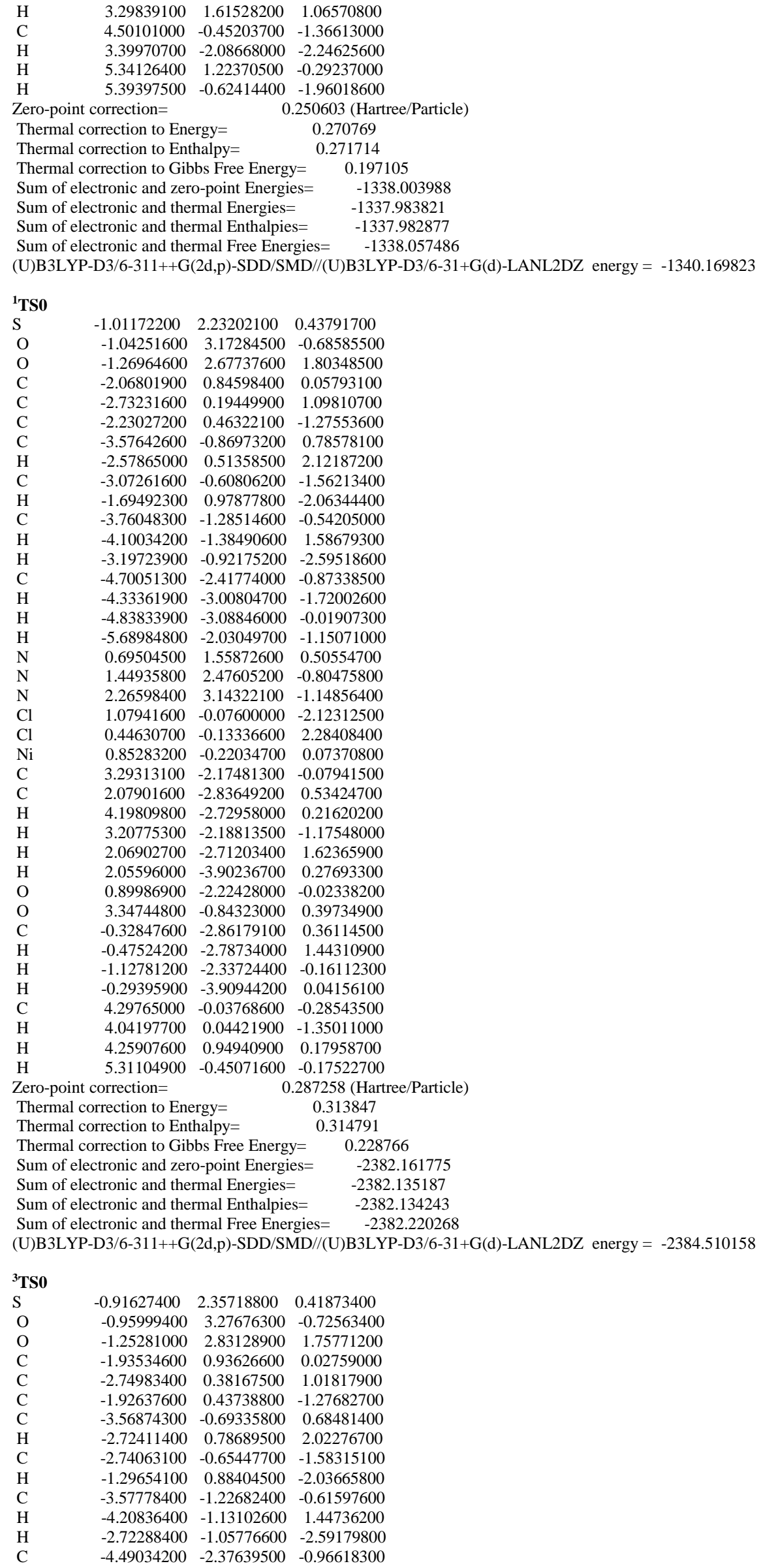


$\begin{array}{lrrr}\mathrm{H} & -4.57639700 & -3.08780200 & -0.13728800 \\ \mathrm{H} & -5.50235500 & -2.01317800 & -1.18846800 \\ \mathrm{H} & -4.13342300 & -2.91682000 & -1.84871500 \\ \mathrm{~N} & 0.75372100 & 1.68038800 & 0.48575700 \\ \mathrm{~N} & 1.49949400 & 2.56353500 & -0.83526500 \\ \mathrm{~N} & 2.40391800 & 2.98599700 & -1.31935500 \\ \mathrm{Cl} & 1.22442800 & -0.34923500 & -2.26892100 \\ \mathrm{Cl} & 0.32982600 & 0.04969800 & 2.26250300 \\ \mathrm{Ni} & 0.86668600 & -0.32507000 & -0.00376400 \\ \mathrm{C} & 3.06682600 & -2.19789900 & 0.10304100 \\ \mathrm{C} & 1.91081700 & -2.96879900 & 0.70290400 \\ \mathrm{H} & 4.01583000 & -2.55385300 & 0.52368400 \\ \mathrm{H} & 3.07872900 & -2.28954400 & -0.98956200 \\ \mathrm{H} & 1.87865100 & -2.82975100 & 1.79129800 \\ \mathrm{H} & 2.00000500 & -4.03689200 & 0.46658100 \\ \mathrm{O} & 0.70582000 & -2.45642600 & 0.12180800 \\ \mathrm{O} & 2.87916600 & -0.82312400 & 0.46616800 \\ \mathrm{C} & -0.48810300 & -3.00811400 & 0.69009400 \\ \mathrm{H} & -0.55590900 & -2.75538800 & 1.75371200 \\ \mathrm{H} & -1.32312400 & -2.56194700 & 0.15060100 \\ \mathrm{H} & -0.48745900 & -4.09521300 & 0.54719700 \\ \mathrm{C} & 3.91159600 & 0.05083100 & -0.00880000 \\ \mathrm{H} & 3.89752300 & 0.09390000 & -1.10264800 \\ \mathrm{H} & 3.69993100 & 1.03142700 & 0.41748000 \\ \mathrm{H} & 4.88089500 & -0.30720200 & 0.35670600 \\ \mathrm{Ze} & \mathrm{H}-\mathrm{p}\end{array}$

$\begin{array}{rrr}4.88089500 & -0.30720200 & 0.35670600 \\ \text { Zero-point correction }= & 0.287666 \text { (Hartreed }\end{array}$

Thermal correction to Energy= $\quad 0.314227$

Thermal correction to Enthalpy= $\quad 0.315171$

Thermal correction to Gibbs Free Energy $=\quad 0.228491$

Sum of electronic and zero-point Energies $=\quad-2382.183541$

Sum of electronic and thermal Energies $=\quad-2382.156980$

Sum of electronic and thermal Enthalpies $=\quad-2382.156035$

Sum of electronic and thermal Free Energies $=\quad-2382.242716$

(U)B3LYP-D3/6-311++G(2d,p)-SDD/SMD//(U)B3LYP-D3/6-31+G(d)-LANL2DZ energy = -2384.5405359

\begin{tabular}{|c|c|c|c|}
\hline \multicolumn{4}{|c|}{${ }^{2}$ INT7 } \\
\hline $\mathrm{C}$ & -0.21004200 & 3.61515000 & 0.32231000 \\
\hline $\mathrm{C}$ & -0.37701900 & 3.27263800 & -1.13891100 \\
\hline $\mathrm{H}$ & -0.04931700 & 4.69527000 & 0.44222400 \\
\hline $\mathrm{H}$ & -1.10833700 & 3.31659000 & 0.87899200 \\
\hline $\mathrm{H}$ & 0.54155000 & 3.47945500 & -1.69923200 \\
\hline $\mathrm{H}$ & -1.20953500 & 3.85724600 & -1.55658100 \\
\hline $\mathrm{O}$ & -0.68075400 & 1.88306500 & -1.20827100 \\
\hline $\mathrm{O}$ & 0.92150000 & 2.91197500 & 0.84661600 \\
\hline $\mathrm{C}$ & -0.89319100 & 1.41107600 & -2.54594100 \\
\hline $\mathrm{H}$ & -0.00370900 & 1.59378900 & -3.15429100 \\
\hline $\mathrm{H}$ & -1.06913000 & 0.34048900 & -2.45888700 \\
\hline $\mathrm{H}$ & -1.77120900 & 1.91526100 & -2.97045000 \\
\hline $\mathrm{C}$ & 1.01645700 & 3.08104700 & 2.25556600 \\
\hline $\mathrm{H}$ & 0.13333500 & 2.66593300 & 2.75761900 \\
\hline $\mathrm{H}$ & 1.91109100 & 2.55695900 & 2.58666300 \\
\hline $\mathrm{H}$ & 1.11043200 & 4.14665800 & 2.50155000 \\
\hline $\mathrm{C}$ & 2.72690000 & -0.33696800 & 1.20091900 \\
\hline $\mathrm{Ni}$ & 0.89358200 & 0.82853900 & -0.01039200 \\
\hline $\mathrm{S}$ & -1.02609100 & -1.02022500 & 0.71124200 \\
\hline $\mathrm{O}$ & -0.86274300 & -2.21253600 & 1.57055900 \\
\hline $\mathrm{O}$ & -0.31659600 & -1.00539000 & -0.61858400 \\
\hline $\mathrm{C}$ & -2.77664400 & -0.89405300 & 0.36255700 \\
\hline $\mathrm{C}$ & -3.29440700 & 0.29649400 & -0.15394900 \\
\hline $\mathrm{C}$ & -3.59725400 & -1.99597900 & 0.58915100 \\
\hline $\mathrm{C}$ & -4.65129800 & 0.36947100 & -0.44880800 \\
\hline $\mathrm{H}$ & -2.63297900 & 1.13983300 & -0.31532700 \\
\hline $\mathrm{C}$ & -4.95646300 & -1.90322600 & 0.28786400 \\
\hline $\mathrm{H}$ & -3.17162700 & -2.90278900 & 1.00353200 \\
\hline $\mathrm{C}$ & -5.50256000 & -0.72675600 & -0.23648800 \\
\hline $\mathrm{H}$ & -5.06300600 & 1.29358900 & -0.84809400 \\
\hline $\mathrm{H}$ & -5.60247300 & -2.75891900 & 0.46605500 \\
\hline $\mathrm{C}$ & -6.96887700 & -0.63417000 & -0.57993800 \\
\hline $\mathrm{H}$ & -7.41281800 & 0.28912200 & -0.19047600 \\
\hline $\mathrm{H}$ & -7.11941800 & -0.63099700 & -1.66713700 \\
\hline $\mathrm{H}$ & -7.53000600 & -1.47952600 & -0.17051500 \\
\hline $\mathrm{N}$ & -0.52509500 & 0.36410700 & 1.36342500 \\
\hline $\mathrm{H}$ & -0.45585600 & 0.27487200 & 2.37428100 \\
\hline $\mathrm{Cl}$ & 2.33516700 & 1.40313700 & -1.72049200 \\
\hline $\mathrm{C}$ & 2.14833000 & -0.64906000 & 2.56287000 \\
\hline $\mathrm{H}$ & 1.85250300 & 0.26601800 & 3.08421500 \\
\hline $\mathrm{H}$ & 1.29608900 & -1.32553800 & 2.54211800 \\
\hline $\mathrm{H}$ & 2.93725200 & -1.12131800 & 3.17115400 \\
\hline $\mathrm{C}$ & 3.78159000 & 0.74729200 & 1.24045100 \\
\hline $\mathrm{H}$ & 3.94896600 & 1.21543600 & 0.27112500 \\
\hline
\end{tabular}




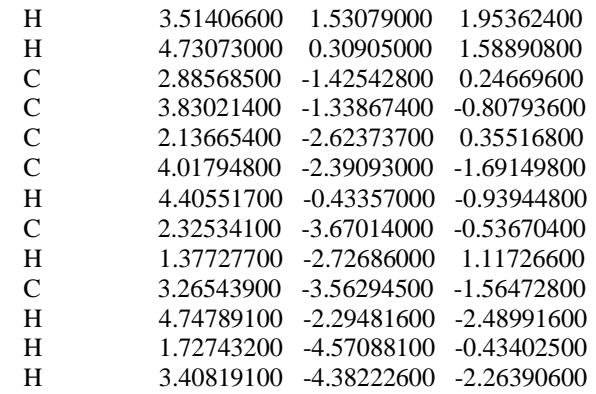

Thermal correction to Energy= $\quad 0.499614$

Thermal correction to Enthalpy= $\quad 0.500558$

Thermal correction to Gibbs Free Energy $=0.401471$

Sum of electronic and zero-point Energies $=\quad-2162.555901$

Sum of electronic and thermal Energies $=\quad-2162.523623$

Sum of electronic and thermal Enthalpies $=\quad-2162.522679$

Sum of electronic and thermal Free Energies $=\quad-2162.621765$

(U)B3LYP-D3/6-311++G(2d,p)-SDD/SMD//(U)B3LYP-D3/6-31+G(d)-LANL2DZ energy = -2165.1111481

\begin{tabular}{|c|c|c|c|}
\hline & & & \\
\hline $\mathrm{C}$ & 2.20369300 & -2.15496700 & -0.92055900 \\
\hline $\mathrm{C}$ & 1.17291900 & -1.92606600 & -2.00310800 \\
\hline $\mathrm{H}$ & 2.83131500 & -3.01897900 & -1.17917400 \\
\hline $\mathrm{H}$ & 2.83941200 & -1.27283800 & -0.79326100 \\
\hline $\mathrm{H}$ & 0.60925300 & -2.84741500 & -2.20827500 \\
\hline $\mathrm{H}$ & 1.66578100 & -1.59564900 & -2.92718800 \\
\hline $\mathrm{O}$ & 0.28601800 & -0.91411800 & -1.54172700 \\
\hline $\mathrm{O}$ & 1.48841400 & -2.41396300 & 0.28464400 \\
\hline $\mathrm{C}$ & -0.70099300 & -0.53464100 & -2.50801200 \\
\hline $\mathrm{H}$ & -1.34987600 & -1.38503100 & -2.74058300 \\
\hline $\mathrm{H}$ & -1.27013100 & 0.27587800 & -2.06459200 \\
\hline $\mathrm{H}$ & -0.19855600 & -0.17781600 & -3.41485900 \\
\hline $\mathrm{C}$ & 2.33507100 & -2.75203100 & 1.38943800 \\
\hline $\mathrm{H}$ & 3.05942200 & -1.94797600 & 1.56489300 \\
\hline $\mathrm{H}$ & 1.67665500 & -2.86439500 & 2.24921100 \\
\hline $\mathrm{H}$ & 2.86096600 & -3.68977300 & 1.17197300 \\
\hline $\mathrm{C}$ & -2.98126900 & 1.40754800 & 0.91338000 \\
\hline $\mathrm{Ni}$ & -0.15858500 & -1.05584700 & 0.53276600 \\
\hline $\mathrm{S}$ & 0.21500400 & 2.07866200 & 0.13584200 \\
\hline $\mathrm{O}$ & 0.24293700 & 3.23994600 & 1.04415700 \\
\hline $\mathrm{O}$ & -0.31772400 & 2.24298700 & -1.22949400 \\
\hline $\mathrm{C}$ & 1.91691600 & 1.52412800 & -0.05910100 \\
\hline $\mathrm{C}$ & 2.64525100 & 1.11426500 & 1.05978600 \\
\hline $\mathrm{C}$ & 2.50610900 & 1.54670100 & -1.31984500 \\
\hline $\mathrm{C}$ & 3.97322700 & 0.72610900 & 0.90603300 \\
\hline $\mathrm{H}$ & 2.17736300 & 1.09395600 & 2.03904200 \\
\hline $\mathrm{C}$ & 3.84049600 & 1.16094600 & -1.45934700 \\
\hline $\mathrm{H}$ & 1.91489500 & 1.86296100 & -2.17161700 \\
\hline $\mathrm{C}$ & 4.59432300 & 0.74934700 & -0.35332000 \\
\hline $\mathrm{H}$ & 4.54190800 & 0.40814300 & 1.77649700 \\
\hline $\mathrm{H}$ & 4.30439900 & 1.18244500 & -2.44244800 \\
\hline $\mathrm{C}$ & 6.04402500 & 0.35787300 & -0.50038400 \\
\hline $\mathrm{H}$ & 6.29471600 & 0.13280700 & -1.54164000 \\
\hline $\mathrm{H}$ & 6.70511100 & 1.16947900 & -0.17052900 \\
\hline $\mathrm{H}$ & 6.28571200 & -0.52127200 & 0.10719800 \\
\hline $\mathrm{N}$ & -0.57473000 & 0.82722200 & 0.86296200 \\
\hline $\mathrm{H}$ & -0.51311900 & 0.97803400 & 1.87107500 \\
\hline $\mathrm{Cl}$ & -0.71663000 & -1.90104200 & 2.61313800 \\
\hline $\mathrm{C}$ & -3.00775500 & 2.86132200 & 0.55035000 \\
\hline $\mathrm{H}$ & -4.03685700 & 3.24903000 & 0.62939600 \\
\hline $\mathrm{H}$ & -2.38230800 & 3.43828100 & 1.23503400 \\
\hline $\mathrm{H}$ & -2.64398100 & 3.04590100 & -0.46135400 \\
\hline $\mathrm{C}$ & -3.01949200 & 1.09417200 & 2.37998500 \\
\hline $\mathrm{H}$ & -2.59280300 & 0.12039800 & 2.63046900 \\
\hline $\mathrm{H}$ & -2.49320000 & 1.86312300 & 2.95362600 \\
\hline $\mathrm{H}$ & -4.06698900 & 1.09357300 & 2.72219600 \\
\hline $\mathrm{C}$ & -3.34039500 & 0.40467400 & -0.05465800 \\
\hline $\mathrm{C}$ & -3.20703500 & -0.97707300 & 0.23949400 \\
\hline $\mathrm{C}$ & -3.81852600 & 0.75637900 & -1.34207800 \\
\hline $\mathrm{C}$ & -3.53823000 & -1.94516800 & -0.70582500 \\
\hline $\mathrm{H}$ & -2.84546000 & -1.29703800 & 1.20814600 \\
\hline $\mathrm{C}$ & -4.17488300 & -0.21549900 & -2.26730000 \\
\hline $\mathrm{H}$ & -3.93135500 & 1.80149300 & -1.60512400 \\
\hline $\mathrm{C}$ & -4.03321300 & -1.57471300 & -1.95830800 \\
\hline $\mathrm{H}$ & -3.42052700 & -2.99488400 & -0.45104900 \\
\hline $\mathrm{H}$ & -4.56300100 & 0.08387800 & -3.23698900 \\
\hline
\end{tabular}


$\begin{array}{ll}\text { Zero-point correction }= & 0.466779 \text { (Hartree/Particle) }\end{array}$

Thermal correction to Energy= $\quad 0.498770$

Thermal correction to Enthalpy= $\quad 0.499715$

Thermal correction to Gibbs Free Energy= 0.401985

Sum of electronic and zero-point Energies $=\quad-2162.546307$

Sum of electronic and thermal Energies $=\quad-2162.514315$

Sum of electronic and thermal Enthalpies $=\quad-2162.513371$

Sum of electronic and thermal Free Energies $=\quad-2162.611100$

(U)B3LYP-D3/6-311++G(2d,p)-SDD/SMD//(U)B3LYP-D3/6-31+G(d)-LANL2DZ energy = -2165.1013484 


\section{References}

(1) (a) Becke, A. D. Density-Functional Thermochemistry. III. The Role of Exact Exchange. J. Chem. Phys. 1993, 98, 5648-5652. (b) Lee, C.; Yang, W.; Parr, R. G. Development of the Colle-Salvetti CorrelationEnergy Formula into a Functional of the Electron Density. Phys. Rev. B, 1988, 37, 785-789. (c) Grimme, S.; Antony, J.; Ehrlich, S.; Krieg, H. A Consistent and Accurate ab initio Parametrization of Density Functional Dispersion Correction (DFT-D) for the 94 Elements H-Pu. J. Chem. Phys. 2010, 132, 154104-154118.

(2) Hay, P. J.; Wadt, W. R. Ab initio Effective Core Potentials for Molecular Calculations. Potentials for K to Au Including the Outermost Core Orbitals. J. Chem. Phys. 1985, 82, 299-310.

(3) Hariharan, P. C.; Pople, J. A. The Influence of Polarization Functions on Molecular Orbital Hydrogenation Energies. Theor. Chim. Acta. 1973, 28, 213-222.

(4) Fukui, K. The Path of Chemical Reactions-the IRC Approach. Acc. Chem. Res. 1981, 14, 363-368.

(5) Marenich, A. V.; Cramer, C. J.; Truhlar, D. G. Universal Solvation Model Based on Solute Electron Density and on a Continuum Model of the Solvent Defined by the Bulk Dielectric Constant and Atomic Surface Tensions. J. Phys. Chem. B 2009, 113, 6378-6396.

(6) (a) Dolg, M.; Wedig, U.; Stoll, H.; Preuss, H. Energy-Adjusted ab initio Pseudopotentials for the First Row Transition Elements. J. Chem. Phys. 1987, 86, 866-872. (b) Andrae, D.; Häußermann, U.; Dolg, M.; Stoll, H.; Preuss, H. Energy-Adjusted ab initio Pseudopotentials for the Second and Third Row Transition Elements. Theor. Chim. Acta. 1990, 77, 123-141.

(7) Frisch, M. J.; Trucks, G. W.; Schlegel, H. B.; Scuseria, G. E.; Robb, M. A.; Cheeseman, J. R.; Scalmani,

G.; Barone, V.; Mennucci, B.; Petersson, G. A.; Nakatsuji, H.; Caricato, M.; Li, X.; Hratchian, H. P.; Izmaylov, A. F.; Bloino, J.; Zheng, G.; Sonnenberg, J. L.; Hada, M.; Ehara, M.; Toyota, K.; Fukuda, R.; Hasegawa, J.; Ishida, M.; Nakajima, T.; Honda, Y.; Kitao, O.; Nakai, H.; Vreven, T.; Montgomery, J. A.; Peralta, J. E.; Ogliaro, F.; Bearpark, M.; Heyd, J. J.; Brothers, E.; Kudin, K. N.; Staroverov, V. N.; Kobayashi, R.; Normand, J.; Raghavachari, K.; Rendell, A.; Burant, J. C.; Iyengar, S. S.; Tomasi, J.; Cossi, M.; Rega, N.; Millam, N. J.; Klene, M.; Knox, J. E.; Cross, J. B.; Bakken, V., Adamo, C., Jaramillo, J., Gomperts, R., Stratmann, R. E., Yazyev, O., Austin, A. J., Cammi, R., Pomelli, C.; Ochterski, J. W.; Martin, R. L.; Morokuma, K.; Zakrzewski, V. G.; Voth, G. A.; Salvador, P.; Dannenberg, J. J.; Dapprich, S.; Daniels, A. D.; Farkas, O.; Foresman, J. B.; Ortiz, J. V.; Cioslowski, J.; Fox, D. J. Gaussian 09, Revision C.01, Gaussian, Inc., Wallingford CT, 2010.

(8) Marcus, R. A. Chemical and Electrochemical Electron-Transfer Theory. Annu. Rev. Phys. Chem. 1964, $15,155-196$.

(9) (a) Dai, J.-J.; Zhang, W.-M.; Shu, Y.-J.; Sun, Y.-Y.; Xu, J.; Feng, Y.-S.; Xu, H.-J. Deboronative 
Cyanation of Potassium Alkyltrifluoroborates via Photoredox Catalysis. Chem. Commun. 2016, 52, 67936796. (b) Huang, H.-C.; Zhang, G.-J.; Gong, L.; Zhang, S.-Y.; Chen, Y.-Y. Visible-Light-Induced Chemoselective Deboronative Alkynylation under Biomolecule-Compatible Conditions. J. Am. Chem. Soc. 2014, 136, 2280-2283.

(10) Thorpe, S. B.; Calderone, J. A.; Santos, W. L. Unexpected Copper(II) Catalysis: Catalytic Amine Base Promoted $\beta$-Borylation of $\alpha, \beta$-Unsaturated Carbonyl Compounds in Water. Org. Lett. 2012, 14, 1918-1921. (11) (a) Yang, C.-T.; Zhang, Z.-Q.; Tajuddin, H.; Wu, C.-C.; Liang, J.; Liu, J.-H.; Fu, Y.; Czyzewska, M.; Steel, P. G.; Marder, T. B.; Liu, L. Alkylboronic Esters from Copper-Catalyzed Borylation of Primary and Secondary Alkyl Halides and Pseudohalides. Angew. Chem., Int. Ed. 2012, 51, 528-532. (b) Fleury-Brégeot, N.; Presset, M.; Beaumard, F.; Colombel, V.; Oehlrich, D.; Rombouts, F.; Molander, G. A. Suzuki-Miyaura Cross-Coupling of Potassium Alkoxyethyltrifluoroborates: Access to Aryl/Heteroarylethyloxy Motifs. $J$. Org. Chem. 2012, 77, 10399-10408.

(12) (a) Tellis, J. C.; Primer, D. N.; Molander, G. A. Single-Electron Transmetalation in Organoboron CrossCoupling by Photoredox/Nickel Dual Catalysis. Science 2014, 345, 433-436. b) Li, Y.-J.; Zhou, K.-X.; Wen, Z.-R.; Cao, S.; Shen, X.; Lei, M.; Gong, L. Copper(II)-Catalyzed Asymmetric Photoredox Reactions: Enantioselective Alkylation of Imines Driven by Visible Light. J. Am. Chem. Soc. 2018, 140, 15850-15858.

(13) Atack, T. C.; Lecker, R. M.; Cook S. P. Iron-Catalyzed Borylation of Alkyl Electrophiles. J. Am. Chem. Soc. 2014, 136, 9521-9523.

(14) (a) Jiang, T.; Gu, Z.-Y.; Yin, L.; Wang, S.-Y.; Ji, S.-J. Cobalt(II)-Catalyzed Isocyanide Insertion Reaction with Sulfonyl Azides in Alcohols: Synthesis of Sulfonyl Isoureas. J. Org. Chem. 2017, 82, 79137919. b) Gui, J.; Xie, H.-S.; Jiang, H.-F.; Zeng, W. Visible-Light-Mediated Sulfonylimination of Tertiary Amines with Sulfonylazides Involving $\mathrm{C}_{\mathrm{sp} 3}-\mathrm{C}_{\mathrm{sp} 3}$ Bond Cleavage. Org. Lett. 2019, 21, 2804-2807.

(15) (a) Zhang, Y.; Ge, X.; Lu, H.-J.; Li G.-G. Catalytic Decarboxylative C-N Formation to Generate Alkyl, Alkenyl, and Aryl Amines. Angew. Chem. Int. Ed. 2020, 59, 1845-1852. (b) Lu, H., Subbarayan, V., Tao, J., Zhang, X. P. Cobalt(II)-Catalyzed Intermolecular Benzylic C-H Amination with 2,2,2Trichloroethoxycarbonyl Azide (TrocN 3 ). Organometallics 2010, 29, 389-393.

(16) (a) Wippich, J.; Truchan, N.; Bach T. Rhodium-Catalyzed N-tert-Butoxycarbonyl (Boc) Amination by Directed C-H Bond Activation. Adv. Synth. Catal. 2016, 358, 2083-2087. b) Carpino, L. A.; Carpino, B. A.; Crowley, P. J.; Giza, C. A.; Terry, P. H. t-Butyl Azidoformate. Org. Synth. 1964, 44, 15. 